\title{
The Effect of Turbulence Intensity and Reynolds Number on the Aerodynamic Behaviour of Kiel, Three-Hole, and Seven-Hole Pressure Probes
}

By

\author{
Carmen Andrew Scribner
}

A thesis submitted to the Faculty of Graduate and Postdoctoral

Affairs in partial fulfillment of the requirements

for the degree of

Master of Applied Science

in

Aerospace Engineering

Carleton University

Ottawa, Ontario

(C) 2011

Carmen Andrew Scribner 
Library and Archives

Canada

Published Heritage Branch

395 Wellington Street Ottawa ON K1A ON4 Canada
Bibliothèque et

Archives Canada

Direction du

Patrimoine de l'édition

395, rue Wellington

Ottawa ON K1A ON4

Canada
Your file Votre référence

ISBN: 978-0-494-83069-7

Our file Notre référence

ISBN: $978-0-494-83069-7$
NOTICE:

The author has granted a nonexclusive license allowing Library and Archives Canada to reproduce, publish, archive, preserve, conserve, communicate to the public by telecommunication or on the Internet, loan, distribute and sell theses worldwide, for commercial or noncommercial purposes, in microform, paper, electronic and/or any other formats.

The author retains copyright ownership and moral rights in this thesis. Neither the thesis nor substantial extracts from it may be printed or otherwise reproduced without the author's permission.
AVIS:

L'auteur a accordé une licence non exclusive permettant à la Bibliothèque et Archives Canada de reproduire, publier, archiver, sauvegarder, conserver, transmettre au public par télécommunication ou par l'Internet, prêter, distribuer et vendre des thèses partout dans le monde, à des fins commerciales ou autres, sur support microforme, papier, électronique et/ou autres formats.

L'auteur conserve la propriété du droit d'auteur et des droits moraux qui protège cette thèse. $\mathrm{Ni}$ la thèse ni des extraits substantiels de celle-ci ne doivent être imprimés ou autrement reproduits sans son autorisation.
In compliance with the Canadian Privacy Act some supporting forms may have been removed from this thesis.

While these forms may be included in the document page count, their removal does not represent any loss of content from the thesis.
Conformément à la loi canadienne sur la protection de la vie privée, quelques formulaires secondaires ont été enlevés de cette thèse.

Bien que ces formulaires aient inclus dans la pagination, il n'y aura aucun contenu manquant. 


\section{Abstract}

The aerodynamic behaviour of pressure probes is influenced by the flows they measure. The present study quantifies the effects of turbulence intensity and Reynolds number on the aerodynamic behaviour of Kiel, three-hole, and seven-hole pressure probes. These effects were investigated by collecting data with the probes at turbulence intensities of up to $12 \%$ and probe Reynolds numbers ranging from 1050 to 5150 in a novel calibration rig that used a perforated pipe to generate the turbulent flows. By comparing the results inferred from the probes to the true values, the effects of turbulence intensity and Reynolds number were identified.

The sensitivity of the Kiel probe to flow misalignments varied with turbulence intensity. Although the probe was insensitive to flow angles of less than about $34^{\circ}$ in low-turbulence flows, this angle decreased to about $24^{\circ}$ at turbulence intensities of $12 \%$. These results were independent of the Reynolds number of the flow.

The three- and seven-hole probes were affected by both turbulence intensity and Reynolds number. The most significant of these effects were systematic errors in the results for total and dynamic pressure when reducing data collected at one operating point using a calibration taken at another. A new data reduction procedure that interpolated between multiple probe calibrations taken at different turbulence intensities and Reynolds numbers was developed to account for these effects. 


\section{Acknowledgements}

This thesis would not have been possible without the assistance of many family members, friends, colleagues, and mentors. Too many deserve thanks to acknowledge them all, but a select few who deserve full recognition are listed below.

My graduate supervisor, Dr. Steen Sjolander, deserves thanks for his guidance and patience throughout this process. Without his insight, this thesis would be worth little more than the paper upon which it is written.

My fellow graduate students, especially Gordon MacIsaac, Steven Hall, Farzad Taremi, and Cameron MacLean, deserve thanks for their assistance. When I had questions, they had answers. I wish them the best of luck as they complete their own degrees.

My girlfriend, Heather Whipp, deserves more thanks than I can fit on this page. Without her support, understanding, and advice, I would not have completed this work. Plus, she edited. A lot.

And finally, my mother deserves the most thanks. Thank you mom, I would not be who I am now without you. 


\section{Table of Contents}

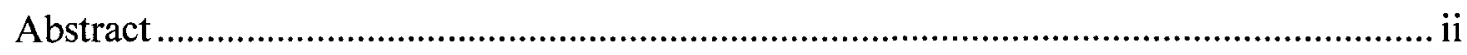

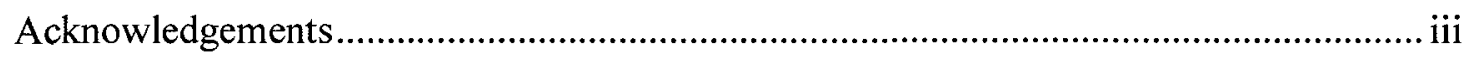

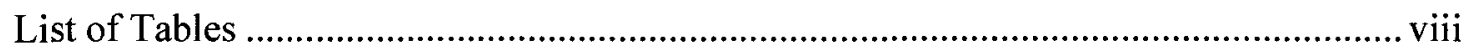

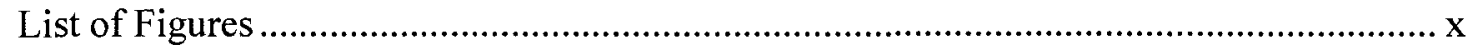

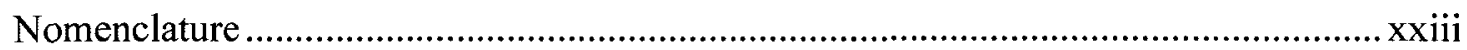

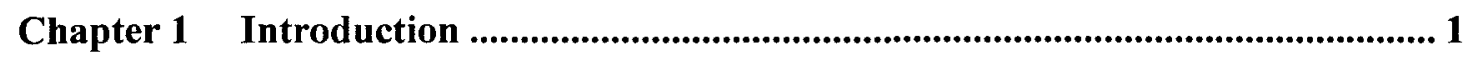

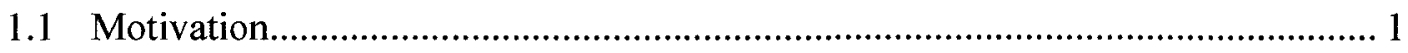

1.2 Thesis Approach and Objectives .............................................................. 2

1.3 Overview of Thesis.................................................................................. 4

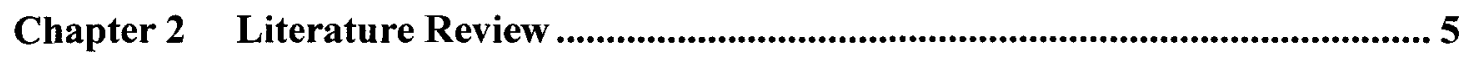

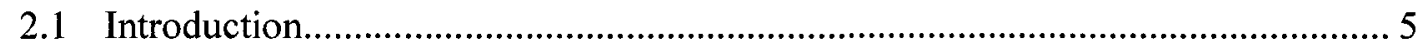

2.2 Factors Influencing Single-Hole Pressure Probe Flow Measurements ................ 6

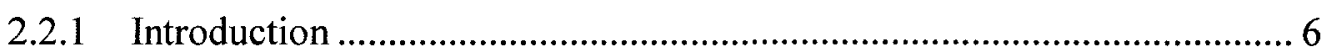

2.2.2 Influence of Reynolds Number .................................................... 7

2.2.3 Influence of Flow Misalignment .................................................. 9

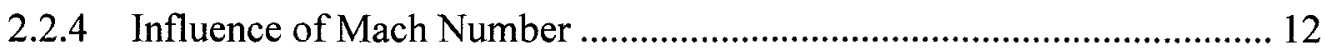

2.2.5 Influence of Velocity Gradient....................................................... 13 
2.2.6 Influence of Turbulence and Unsteadiness ............................................ 14

2.2.7 Influence of Wall Proximity .................................................................. 18

2.3 Factors Influencing Multi-Hole Pressure Probe Flow Measurements.................. 18

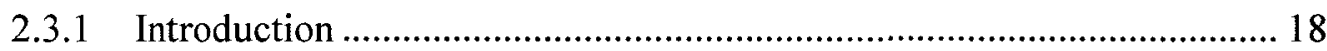

2.3.2 Influence of Reynolds Number ............................................................. 19

2.3.3 Influence of Flow Misalignment .......................................................... 25

2.3.4 Influence of Mach Number ................................................................ 26

2.3.5 Influence of Velocity Gradient................................................................. 26

2.3.6 Influence of Turbulence and Unsteadiness ............................................. 27

2.3.7 Influence of Wall Proximity ................................................................... 29

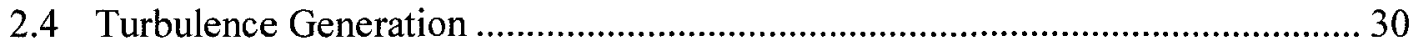

Chapter 3 Experimental Apparatus and Procedures................................................... 33

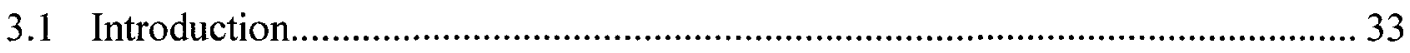

3.2 Low-Turbulence Probe Calibration Rig ............................................................... 33

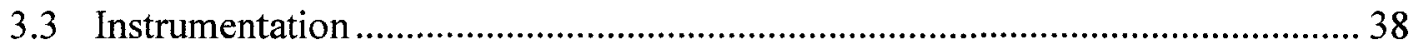

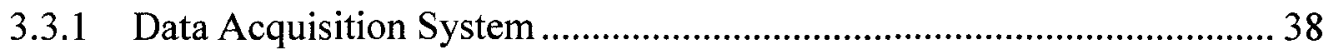

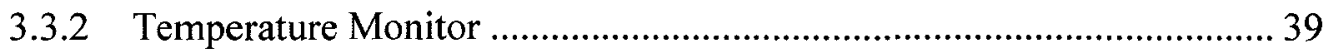

3.3.3 Digital Barometer …………………………………............................ 39

3.3.4 Hot-wire Anemometer and Probes ............................................................ 39

3.3.5 Pressure Transducers ........................................................................ 41

3.3.6 Pressure Probes.................................................................................. 42

3.3.6.1 Kiel Probe ................................................................................ 42 
3.3.6.2 Three-Hole Pressure Probe................................................... 42

3.3.6.3 Seven-Hole Pressure Probes.............................................. 43

3.4 Probe Calibration, Data Reduction, and Measurement Uncertainty.................. 45

3.4.1 Single-Normal Hot-wire Probe ..................................................... 45

3.4.2 X-Wire Type Hot-wire Probe ........................................................... 48

3.4.3 Three-Hole Pressure Probe................................................................ 49

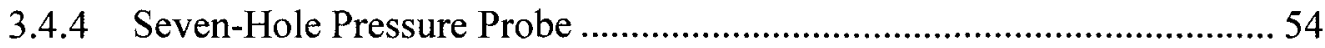

Chapter 4 The Effect of Turbulence Intensity and Reynolds Number on the Aerodynamic Behaviour of Kiel, Three-Hole, and Seven-Hole Pressure Probes ..... 62

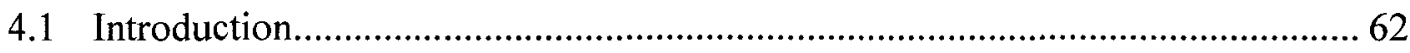

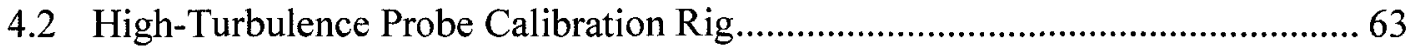

4.3 Effects of Turbulence Intensity and Reynolds Number on Flow Angle

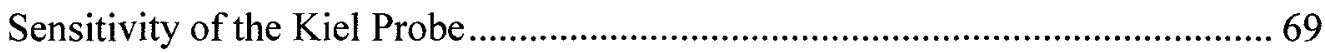

4.4 Effects of Turbulence Intensity and Reynolds Number on Three-Hole

Pressure Probes..................................................................................... 76

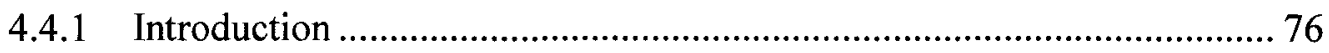

4.4.2 Effects of Turbulence Intensity on Three-Hole Pressure Probe Flow Measurements at Off-Calibration Conditions .................................... 79

4.4.3 Effects of Reynolds Number on Three-Hole Pressure Probe Flow Measurements at Off-Calibration Conditions .................................. 92

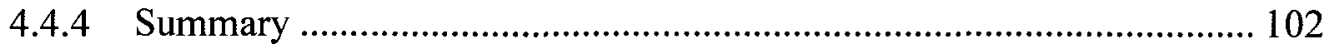


4.5 Effects of Turbulence Intensity and Reynolds Number on Seven-Hole

Pressure Probes 104

4.5.1 Introduction

4.5.2 Effects of Turbulence Intensity on Seven-Hole Pressure Probe Flow Measurements at Off-Calibration Conditions 109

4.5.3 Effects of Reynolds Number on Seven-Hole Pressure Probe Flow Measurements at Off-Calibration Conditions 120

4.5.4 Summary 126

4.6 Method to Account for the Effects of Turbulence Intensity and Reynolds Number on Seven-Hole Pressure Probe Data Reduction

Chapter 5 Conclusions and Recommendations................................................... 136

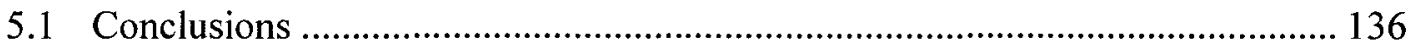

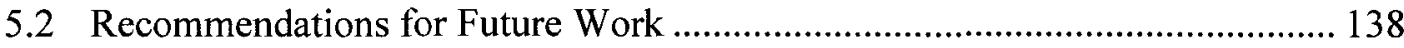

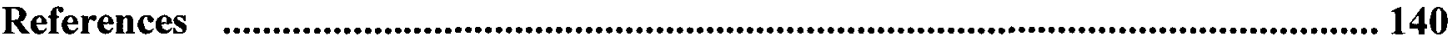

Appendix A: Pressure Transducer Calibration ................................................... 147

Appendix B: Supplemental Results from the Kiel Probe................................... 150

Appendix C: Supplemental Results from the Three-Hole Pressure Probe ............. 152

Appendix D: Supplemental Results from the Seven-Hole Pressure Probe .............. 164

Appendix E: Bi-Linear Interpolative Data Reduction Procedure for Multi-Hole

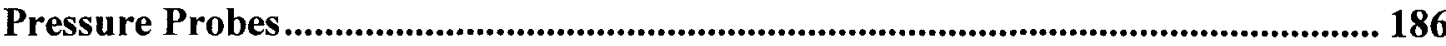




\section{List of Tables}

Table 3.1: X-Wire Hot-wire Probe Measurement Uncertainty, from Buresti and

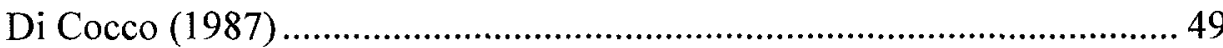

Table 3.2: Three-Hole Pressure Probe Measurement Uncertainty ............................ 53

Table 3.3: $\quad$ Pressure Probe Data Sample Lengths.................................................... 54

Table 3.4: $\quad$ Seven-Hole Pressure Probe Measurement Uncertainty ............................ 61

Table 4.1: High-Turbulence Probe Calibration Rig Hole and Grid Combinations..... 65

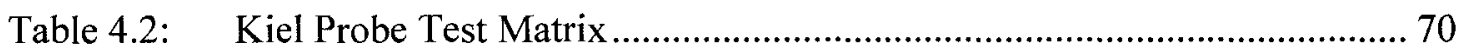

Table 4.3: Three-Hole Pressure Probe Calibration and Validation Test Matrix ......... 78

Table 4.4: $\quad$ Estimated Bias in Three-Hole Probe Total Pressure Results due to

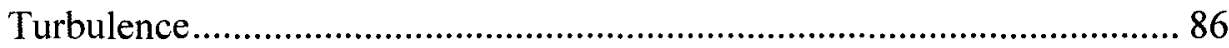

Table 4.5: Values of $\mathrm{C}_{\mathrm{q}} \mathrm{Cal}$ for Different Turbulence Intensities ...............................89

Table 4.6: Summary of the Effects of Turbulence on Three-Hole Pressure Probe Measurements.

Table 4.7: Summary of the Effects of Reynolds Number on Three-Hole Pressure Probe Measurements

Table 4.8: Seven-Hole Pressure Probe Calibration Test Matrix for Investigating the Influence of Turbulence Intensity 105 
Table 4.9: Seven-Hole Pressure Probe Validation Test Matrix for Investigating the Influence of Turbulence Intensity

Table 4.10: Seven-Hole Pressure Probe Calibration Test Matrix for Investigating the Influence of Reynolds Number 106

Table 4.11: Seven-Hole Pressure Probe Validation Test Matrix for Investigating the Influence of Reynolds Number 106

Table 4.12: Estimated Bias in Total Pressure Results due to Turbulence 114

Table 4.13: Values of $\mathrm{C}_{\mathrm{q} \text { Cal }}$ for Different Turbulence Intensities

Table 4.14: Summary of the Effects of Turbulence on Seven-Hole Pressure Probe Measurements

Table 4.15: Summary of the Effects of Reynolds Number on Seven-Hole Pressure Probe Measurements.

Table 4.16: Seven-Hole Pressure Probe Calibrations used to Reduce Data from MacIsaac et al. (2009) 129 


\section{List of Figures}

Figure 2.1: Single-Hole Pressure Probes ........................................................................ 6

Figure 2.2: $\quad$ Pitot Tube Reynolds Number Effects, from MacMillan (1954) ................ 8

Figure 2.3: Static Pressure Probe Flow Misalignment Effects, from Krause and Gettelman (1952)

Figure 2.4: Total Pressure Probe Flow Misalignment Effects, from Chue (1975)...... 10

Figure 2.5: Hemispherical Pitot Probe Flow Misalignment Effects with Various Tip Diameter Ratios, from Ower and Pankhurst (1977) ........................ 11

Figure 2.6: Pitot Probe in a Velocity Gradient .................................................... 14

Figure 2.7: "Standard" Static Pressure Probe Flow Misalignment Effects in Turbulent Flow, from Christiansen and Bradshaw (1981)....................... 15

Figure 2.8: Multi-Hole Pressure Probes ................................................................. 19

Figure 2.9: Three-Hole Pressure Probe Reynolds Number Effects, from Benner (2003)

Figure 2.10: Seven-Hole Pressure Probe Reynolds Number Effects, from MacIsaac et al. (2009).

Figure 2.11: Reynolds Number Effects on a Yaw Hole of a Five-Hole Pressure Probe, from Dominy and Hodson (1993): (a) Contour Interval=0.025; (b) Reynolds Number Interval of $5 \times 10^{3}$ starting at $10 \times 10^{3}$ 
Figure 2.12: Flow Separation on the tip of a Five-Hole Pressure Probe, from Johnson (1985)

Figure 2.13: Five-hole Probe Geometry, from Dominy and Hodson (1993)

Figure 2.14: Turbulence Intensity Effects on the Yaw Coefficient of a Five-Hole Pressure Probe, from Dominy and Hodson (1993) 28

Figure 2.15: Turbulence Generation Upstream of a Flow Convergence, from Matsunuma et al. (1999) 31

Figure 3.1: Schematic Diagram of the Low-Turbulence Probe Calibration Rig, adapted from Benner (2003) 34

Figure 3.2: Profile of Total Pressure across the Calibration Jet at a Low Turbulence Intensity. 36

Figure 3.3: Probe Tangential Coordinate System, adapted from Gerner et al. (1984)

Figure 3.4: Probe Polar Coordinate System, adapted from Gerner et al. (1984)...... 37

Figure 3.5: Constant Temperature Anemometer Block Diagram, from Mahallati (2003) 40

Figure 3.6: Hot-wire Probes 41

Figure 3.7: $\quad$ Modified Kiel Probe, adapted from Taremi (2005) 42

Figure 3.8: Three-hole Pressure Probe 43

Figure 3.9: $\quad$ Seven-Hole Pressure Probe 7HP1 44

Figure 3.10: Seven-Hole Pressure Probe 7HP2 44

Figure 3.11: Sample Calibration Curves for Single-Normal Hot-wire Probes 46 
Figure 3.12: Sample Autocorrelation Function

Figure 3.13: Three-Hole Pressure Probe Port Numbering Convention ........................ 51

Figure 3.14: Sample $K_{\phi 2}$ and $K_{\phi 3}$ Calibration Curves for the Three-Hole Pressure Probe 51

Figure 3.15: Sample $\mathrm{K}_{12}$ and $\mathrm{K}_{13}$ Calibration Curves for the Three-Hole Pressure Probe. 51

Figure 3.16: Sample $\mathrm{K}_{1}$ Calibration Curve for the Three-hole Pressure Probe 52

Figure 3.17: Seven-Hole Pressure Probe Port Numbering Convention. 55

Figure 3.18: Sample Yaw Coefficient Calibration Surface for the Seven-Hole Pressure Probe (7HP1) 57

Figure 3.19: Sample Pitch Coefficient Calibration Surface for the Seven-Hole Pressure Probe (7HP1) 58

Figure 3.20: Sample Total Pressure Coefficient Calibration Surface for the SevenHole Pressure Probe (7HP1)

Figure 3.21: Sample Dynamic Pressure Calibration Surface for the Seven-Hole Pressure Probe (7HP1) 60

Figure 4.1: Schematic Diagram of the High-Turbulence Calibration Rig 63

Figure 4.2: Profile of Total Pressure across the Calibration Jet at an Elevated Turbulence Intensity. 67

Figure 4.3: Profile of Velocity across the Calibration Jet at an Elevated Turbulence Intensity. 68

Figure 4.4: Profile of Streamwise Turbulence Intensity across the Calibration Jet at an Elevated Turbulence Intensity 68 
Figure 4.5: Kiel Probe Results for Total Pressure at Various Reynolds Numbers and $\mathrm{Tu}=1.5 \%$

Figure 4.6: Kiel Probe Results for Total Pressure at Various Reynolds Numbers and $\mathrm{Tu}=5 \%$

Figure 4.7: Kiel Probe Results for Total Pressure at Various Reynolds Numbers and $\mathrm{Tu}=12 \%$ 74

Figure 4.8: Kiel Probe Results for Total Pressure at $\mathrm{Re}_{\mathrm{D}}=3000$ and Various Turbulence Intensities 75

Figure 4.9: Kiel Probe Results for Critical Angle as a function of Turbulence Intensity 75

Figure 4.10: Three-Hole Pressure Probe Results for Flow Angle using Calibration and Validation Data at $\operatorname{Re}_{\mathrm{D}}=2500, \mathrm{Tu}=1.5 \%$

Figure 4.11: Three-Hole Pressure Probe Flow Angle Error due to Turbulence Intensity using a Calibration at $\operatorname{Re}_{\mathrm{D}}=2500, \mathrm{Tu}=1.5 \%$ and Validation Data at $\operatorname{Re}_{\mathrm{D}}=2500, \mathrm{Tu}=5 \%$ 81

Figure 4.12: Three-Hole Pressure Probe Flow Angle Error due to Turbulence Intensity using a Calibration at $\mathrm{Re}_{\mathrm{D}}=2500, \mathrm{Tu}=1.5 \%$ and Validation Data at $\operatorname{Re}_{\mathrm{D}}=2500, \mathrm{Tu}=12 \%$

Figure 4.13: Three-Hole Pressure Probe Total Pressure Error due to Turbulence Intensity using a Calibration at $\mathrm{Re}_{\mathrm{D}}=2500, \mathrm{Tu}=1.5 \%$ 82

Figure 4.14: Three-Hole Pressure Probe RMS Total Pressure Error due to Turbulence Intensity using a Calibration at $\operatorname{Re}_{\mathrm{D}}=2500$ and $\mathrm{Tu}=1.5 \%$ 84

Figure 4.15: Three-Hole Pressure Probe Maximum Total Pressure Error due to Turbulence Intensity using a Calibration at $\mathrm{Re}_{\mathrm{D}}=2500$ and $\mathrm{Tu}=1.5 \% \ldots \ldots .84$ 
Figure 4.16: Three-Hole Pressure Probe Dynamic Pressure Error due to Turbulence Intensity using a Calibration at $\mathrm{Re}_{\mathrm{D}}=2500, \mathrm{Tu}=1.5 \%$.

Figure 4.17: Effect of Uncertainty in $\mathrm{C}_{\mathrm{q} \text { Cal }}$ on Three-Hole Probe Dynamic Pressure Results using a Calibration at $\operatorname{Re}_{\mathrm{D}}=1850, \mathrm{Tu}=1.5 \%$ and Validation data at $\operatorname{Re}_{\mathrm{D}}=1850, \mathrm{Tu}=12 \%$

Figure 4.18: Three-Hole Pressure Probe Dynamic Pressure Error due to Turbulence Intensity using a Calibration at $\mathrm{Re}_{\mathrm{D}}=2500, \mathrm{Tu}=1.5 \%$, Modified to $\Delta \mathrm{C}_{\mathrm{q}}=0$ at $\alpha=0$.

Figure 4.19: Three-Hole Pressure Probe RMS Dynamic Pressure Error due to Turbulence Intensity using a Calibration at $\mathrm{Re}_{\mathrm{D}}=2500$ and $\mathrm{Tu}=1.5 \%$

Figure 4.20: Three-Hole Pressure Probe Maximum Dynamic Pressure Error due to Turbulence Intensity using a Calibration at $\mathrm{Re}_{\mathrm{D}}=2500$ and $\mathrm{Tu}=1.5 \% \ldots \ldots .92$

Figure 4.21: Three-Hole Pressure Probe Results for Flow Angle using Calibration and Validation Data at $\operatorname{Re}_{\mathrm{D}}=4100, \mathrm{Tu}=1.5 \%$

Figure 4.22: Three-Hole Pressure Probe Flow Angle Error due to Reynolds Number using a Calibration at $\operatorname{Re}_{\mathrm{D}}=4100, \mathrm{Tu}=1.5 \%$ and Validation Data at $\operatorname{Re}_{\mathrm{D}}=2500, \mathrm{Tu}=1.5 \%$ 94

Figure 4.23: Three-Hole Pressure Probe Flow Angle Error due to Reynolds Number using a Calibration at $\mathrm{Re}_{\mathrm{D}}=4100, \mathrm{Tu}=1.5 \%$ and Validation Data at $\operatorname{Re}_{\mathrm{D}}=1850, \mathrm{Tu}=1.5 \%$ 94

Figure 4.24: Three-Hole Pressure Probe Flow Angle Results for Various Reynolds Numbers, from Benner (2003) 95

Figure 4.25: Three-Hole Pressure Probe Flow Angle Error due to Turbulence Intensity using a Calibration at $\mathrm{ReD}=2500, \mathrm{Tu}=1.5 \%$ and Validation Data at $\mathrm{ReD}=2500, \mathrm{Tu}=12 \%$ 
Figure 4.26: Comparison of the Three-Hole Probes from the Present Study and Benner (2003).

Figure 4.27: Three-Hole Pressure Probe Total Pressure Error due to Reynolds Number using a Calibration at $\mathrm{Re}_{\mathrm{D}}=4100, \mathrm{~T} u=1.5 \%$

Figure 4.28: Three-Hole Pressure Probe Total Pressure Results for Various Reynolds Numbers, from Benner (2003)

Figure 4.29: Three-Hole Pressure Probe Dynamic Pressure Error due to Reynolds Number using a Calibration at $\mathrm{Re}_{\mathrm{D}}=4100, \mathrm{Tu}=1.5 \%$.

Figure 4.30: Three-Hole Pressure Probe Dynamic Pressure Results for Various Reynolds Numbers, from Benner (2003).

Figure 4.31: Three-Hole Pressure Probe Dynamic Pressure Error due to Reynolds Number using a Calibration at $\operatorname{Re}_{\mathrm{D}}=4100$ and $\mathrm{Tu}=1.5 \%$ 102

Figure 4.32: Probe Tangential Coordinate System, adapted from Gerner et al. (1984) 110

Figure 4.33: Probe Polar Coordinate System, adapted from Gerner et al. (1984) 110

Figure 4.34: Seven-Hole Pressure Probe Flow Angle Error due to Turbulence Intensity using a Calibration at $\mathrm{Re}_{\mathrm{D}}=3900, \mathrm{Tu}=1.5 \%$.

Figure 4.35: Seven-Hole Pressure Probe Total Pressure Error due to Turbulence Intensity using a Calibration at $\mathrm{Re}_{\mathrm{D}}=3900, \mathrm{Tu}=1.5 \%$.

Figure 4.36: Seven-Hole Pressure Probe Total Pressure Error due to Turbulence Intensity using a Calibration at $\mathrm{Re}_{\mathrm{D}}=3900, \mathrm{Tu}=1.5 \%$ and Validation Data at $\operatorname{Re}_{\mathrm{D}}=3900, \mathrm{Tu}=12 \%$.

Figure 4.37: Seven-Hole Pressure Probe Maximum Total Pressure Error due to Turbulence Intensity using a Calibration at $\mathrm{Re}_{\mathrm{D}}=3900$ and $\mathrm{Tu}=1.5 \% \ldots . .114$ 
Figure 4.38: Seven-Hole Pressure Probe Dynamic Pressure Error due to Turbulence Intensity using a Calibration at $\mathrm{Re}_{\mathrm{D}}=3900, \mathrm{Tu}=1.5 \% \ldots \ldots \ldots 115$

Figure 4.39: Seven-Hole Pressure Probe Dynamic Pressure Error due to Turbulence Intensity using a Calibration at $\mathrm{Re}_{\mathrm{D}}=3900, \mathrm{Tu}=1.5 \%$ and Validation Data at $\operatorname{Re}_{\mathrm{D}}=3900, \mathrm{Tu}=12 \%$ 116

Figure 4.40: Effect of Uncertainty in $\mathrm{C}_{\mathrm{qCal}}$ on Seven-Hole Probe Dynamic Pressure Results using a Calibration at $\operatorname{Re}_{\mathrm{D}}=3900, \mathrm{Tu}=1.5 \%$ and Validation data at $\operatorname{Re}_{\mathrm{D}}=3900, \mathrm{Tu}=12 \%$ 118

Figure 4.41: Cut-Planes of the Dynamic Pressure Calibration Coefficient for the Seven-Hole Pressure Probe at a Reynolds Number of 3900 and Various Turbulence Intensities 119

Figure 4.42: Seven-Hole Pressure Probe Flow Angle Error due to Reynolds Number using a Calibration at $\operatorname{Re}_{\mathrm{D}}=5150, \mathrm{Tu}=1.5 \%$

Figure 4.43: Seven-Hole Pressure Probe Total Pressure Error due to Reynolds Number using a Calibration at $\operatorname{Re}_{\mathrm{D}}=5150, \mathrm{Tu}=1.5 \%$

Figure 4.44: Seven-Hole Pressure Probe Total Pressure Error due to Reynolds Number using a Calibration at $\operatorname{Re}_{\mathrm{D}}=5150, \mathrm{Tu}=1.5 \%$ and Validation Data at $\operatorname{Re}_{\mathrm{D}}=2500, \mathrm{Tu}=1.5 \%$

Figure 4.45: Seven-Hole Pressure Probe Dynamic Pressure Error due to Reynolds Number using a Calibration at $\operatorname{Re}_{\mathrm{D}}=5150, \mathrm{Tu}=1.5 \%$

Figure 4.46: Seven-Hole Pressure Probe Dynamic Pressure Error due to Reynolds Number using a Calibration at $\mathrm{Re}_{\mathrm{D}}=5150, \mathrm{Tu}=1.5 \%$ and Validation Data at $\mathrm{Re}_{\mathrm{D}}=2500, \mathrm{Tu}=1.5 \%$

Figure 4.47: Seven-Hole Pressure Probe Dynamic Pressure Error due to Reynolds Number using a Calibration at $\operatorname{Re}_{D}=5150$ and $T u=1.5 \%$ 126 
Figure 4.48: Total Pressure Results using Seven-Hole Probe Data from MacIsaac et al. (2009)

Figure 4.49: Comparison between the Results for Total Pressure from

Interpolating on Reynolds Number at $\mathrm{Tu}=1.5 \%$ and Interpolating on

Both Turbulence Intensity and Reynolds Number.

Figure 4.50: Comparison of Total Pressure Results from the Seven-Hole and Kiel

Probes

Figure A.1: Water Micromanometer, from Benner (2003)

Figure A.2: Sample Transducer Calibration Curve

Figure B.1: Kiel Probe Results for Total Pressure at Various Reynolds Numbers and $\mathrm{Tu}=1.5 \%$

Figure B.2: Kiel Probe Results for Total Pressure at $\mathrm{Re}_{\mathrm{D}}=2200$ and Various

Turbulence Intensities

Figure B.3: Kiel Probe Results for Critical Angle as a function of Turbulence

Intensity

Figure C.1: Three-Hole Pressure Probe Results: Calibration at $\operatorname{Re}_{\mathrm{D}}=1850$, $\mathrm{Tu}=1.5 \%$; Data at $\mathrm{Re}_{\mathrm{D}}=1850, \mathrm{Tu}$ as shown 153

Figure C.2: Three-Hole Pressure Probe Results: Calibration at $\operatorname{Re}_{\mathrm{D}}=2500$, $\mathrm{Tu}=1.5 \%$; Data at $\operatorname{Re}_{\mathrm{D}}=2500$, $\mathrm{Tu}$ as shown

Figure C.3: Three-Hole Pressure Probe Results: Calibration at $\operatorname{Re}_{\mathrm{D}}=1850$, $\mathrm{Tu}=3 \%$; Data at $\mathrm{Re}_{\mathrm{D}}=1850, \mathrm{Tu}$ as shown 154

Figure C.4: Three-Hole Pressure Probe Results: Calibration at $\operatorname{Re}_{\mathrm{D}}=2500$, $\mathrm{Tu}=3 \%$; Data at $\mathrm{Re}_{\mathrm{D}}=2500$, $\mathrm{Tu}$ as shown 154 
Figure C.5: Three-Hole Pressure Probe Results: Calibration at $\operatorname{Re}_{\mathrm{D}}=1850$, $\mathrm{Tu}=5 \%$; Data at $\operatorname{Re}_{\mathrm{D}}=1850$, Tu as shown

Figure C.6: Three-Hole Pressure Probe Results: Calibration at $\operatorname{Re}_{\mathrm{D}}=2500$, $\mathrm{Tu}=5 \%$; Data at $\mathrm{Re}_{\mathrm{D}}=2500$, Tu as shown

Figure C.7: Three-Hole Pressure Probe Results: Calibration at $\operatorname{Re}_{\mathrm{D}}=1850$, $\mathrm{Tu}=8 \%$; Data at $\mathrm{Re}_{\mathrm{D}}=1850, \mathrm{Tu}$ as shown 156

Figure C.8: Three-Hole Pressure Probe Results: Calibration at $\operatorname{Re}_{\mathrm{D}}=2500$, $\mathrm{Tu}=8 \%$; Data at $\mathrm{Re}_{\mathrm{D}}=2500, \mathrm{Tu}$ as shown

Figure C.9: Three-Hole Pressure Probe Results: Calibration at $\operatorname{Re}_{\mathrm{D}}=1850$, $\mathrm{Tu}=12 \%$; Data at $\mathrm{Re}_{\mathrm{D}}=1850, \mathrm{Tu}$ as shown

Figure C.10: Three-Hole Pressure Probe Results: Calibration at $\operatorname{Re}_{\mathrm{D}}=2500$, $\mathrm{Tu}=12 \%$; Data at $\mathrm{Re}_{\mathrm{D}}=2500, \mathrm{Tu}$ as shown

Figure C.11: Three-Hole Pressure Probe Results: Calibration at $\operatorname{Re}_{\mathrm{D}}=1850$, $\mathrm{Tu}=1.5 \%$; Data at $\mathrm{Re}_{\mathrm{D}}$ as shown, $\mathrm{Tu}=1.5 \%$ 158

Figure C.12: Three-Hole Pressure Probe Results: Calibration at $\mathrm{ReD}=4100$, $\mathrm{Tu}=1.5 \%$; Data at $\mathrm{ReD}$ as shown, $\mathrm{Tu}=1.5 \%$ 158

Figure C.13: Three-Hole Pressure Probe Results: Calibration at $\operatorname{Re}_{\mathrm{D}}=1850$, $\mathrm{Tu}=3 \%$; Data at $\mathrm{Re}_{\mathrm{D}}$ as shown, $\mathrm{Tu}=3 \%$

Figure C.14: Three-Hole Pressure Probe Results: Calibration at $\operatorname{Re}_{\mathrm{D}}=2500$, $\mathrm{Tu}=3 \%$; Data at $\mathrm{Re}_{\mathrm{D}}$ as shown, $\mathrm{Tu}=3 \%$ 159

Figure C.15: Three-Hole Pressure Probe Results: Calibration at $\operatorname{Re}_{\mathrm{D}}=1850$, $\mathrm{Tu}=5 \%$; Data at $\mathrm{Re}_{\mathrm{D}}$ as shown, $\mathrm{Tu}=5 \%$ 160

Figure C.16: Three-Hole Pressure Probe Results: Calibration at $\operatorname{Re}_{\mathrm{D}}=3800$, $\mathrm{Tu}=5 \%$; Data at $\mathrm{Re}_{\mathrm{D}}$ as shown, $\mathrm{Tu}=5 \%$ 160 
Figure C.17: Three-Hole Pressure Probe Results: Calibration at $\operatorname{Re}_{\mathrm{D}}=1850$, $\mathrm{Tu}=8 \%$; Data at $\mathrm{Re}_{\mathrm{D}}$ as shown, $\mathrm{Tu}=8 \%$

Figure C.18: Three-Hole Pressure Probe Results: Calibration at $\mathrm{Re}_{\mathrm{D}}=2500$, $\mathrm{Tu}=8 \%$; Data at $\operatorname{Re}_{\mathrm{D}}$ as shown, $\mathrm{Tu}=8 \%$

Figure C.19: Three-Hole Pressure Probe Results: Calibration at $\operatorname{Re}_{\mathrm{D}}=1850$, $\mathrm{Tu}=12 \%$; Data at $\mathrm{Re}_{\mathrm{D}}$ as shown, $\mathrm{Tu}=12 \%$ 162

Figure C.20: Three-Hole Pressure Probe Results: Calibration at $\operatorname{Re}_{\mathrm{D}}=2500$, $\mathrm{Tu}=12 \%$; Data at $\mathrm{Re}_{\mathrm{D}}$ as shown, $\mathrm{Tu}=12 \%$ 162

Figure C.21: Three-Hole Pressure Probe Results: Calibration at $\operatorname{Re}_{\mathrm{D}}=2500$, $\mathrm{Tu}=1.5 \%$; Data at $\mathrm{Re}_{\mathrm{D}}=1850, \mathrm{Tu}=12 \%$

Figure C.22: Three-Hole Pressure Probe Results: Calibration at $\mathrm{Re}_{\mathrm{D}}=1850$, $\mathrm{Tu}=12 \%$; Data at $\mathrm{Re}_{\mathrm{D}}=2500, \mathrm{Tu}=1.5 \%$ 163

Figure D.1: Seven-Hole Pressure Probe Results: Calibration at $\operatorname{Re}_{\mathrm{D}}=2500$, $\mathrm{Tu}=1.5 \%$; Data at $\mathrm{Re}_{\mathrm{D}}=2500, \mathrm{Tu}$ as shown 165

Figure D.2: Seven-Hole Pressure Probe Results: Calibration at $\operatorname{Re}_{\mathrm{D}}=2500$, $\mathrm{Tu}=3 \%$; Data at $\mathrm{Re}_{\mathrm{D}}=2500$, Tu as shown 165

Figure D.3: Seven-Hole Pressure Probe (7HP1) Results: Calibration at $\operatorname{Re}_{\mathrm{D}}=2500, \mathrm{Tu}=5 \%$; Data at $\operatorname{Re}_{\mathrm{D}}=2500$, Tu as shown. 166

Figure D.4: Seven-Hole Pressure Probe (7HP1) Results: Calibration at $\mathrm{Re}_{\mathrm{D}}=2500, \mathrm{Tu}=8 \%$; Data at $\mathrm{Re}_{\mathrm{D}}=2500$, Tu as shown

Figure D.5: Seven-Hole Pressure Probe (7HP1) Results: Calibration at $\operatorname{Re}_{\mathrm{D}}=2500, \mathrm{Tu}=12 \%$; Data at $\mathrm{Re}_{\mathrm{D}}=2500$, Tu as shown

Figure D.6: Seven-Hole Pressure Probe (7HP1) Results: Calibration at $\mathrm{Re}_{\mathrm{D}}=3900, \mathrm{Tu}=1.5 \%$; Data at $\mathrm{Re}_{\mathrm{D}}=3900, \mathrm{Tu}$ as shown 167 
Figure D.7: Seven-Hole Pressure Probe (7HP1) Results: Calibration at $\operatorname{Re}_{\mathrm{D}}=3900, \mathrm{Tu}=3 \%$; Data at $\mathrm{Re}_{\mathrm{D}}=3900, \mathrm{Tu}$ as shown 168

Figure D.8: Seven-Hole Pressure Probe (7HP1) Results: Calibration at $\operatorname{Re}_{\mathrm{D}}=3900, \mathrm{Tu}=5 \%$; Data at $\mathrm{Re}_{\mathrm{D}}=3900$, $\mathrm{Tu}$ as shown 168

Figure D.9: Seven-Hole Pressure Probe (7HP1) Results: Calibration at $\operatorname{Re}_{\mathrm{D}}=3900, \mathrm{Tu}=8 \%$; Data at $\mathrm{Re}_{\mathrm{D}}=3900$, Tu as shown

Figure D.10: Seven-Hole Pressure Probe (7HP1) Results: Calibration at $\operatorname{Re}_{\mathrm{D}}=3900, \mathrm{Tu}=12 \%$; Data at $\operatorname{Re}_{\mathrm{D}}=3900$, Tu as shown 169

Figure D.11: Seven-Hole Pressure Probe (7HP1) Results for Total Pressure: Calibration at $\operatorname{Re}_{\mathrm{D}}=3900, \mathrm{Tu}=1.5 \%$; Data at $\operatorname{Re}_{\mathrm{D}}=3900$, Tu as shown $\ldots . .170$

Figure D.12: Seven-Hole Pressure Probe (7HP1) Results for Dynamic Pressure: Calibration at $\operatorname{Re}_{\mathrm{D}}=3900, \mathrm{Tu}=1.5 \%$; Data at $\mathrm{Re}_{\mathrm{D}}=3900$, $\mathrm{Tu}$ as shown .... 171

Figure D.13: Seven-Hole Pressure Probe (7HP1) Results: Calibration at $\operatorname{Re}_{\mathrm{D}}=2000, \mathrm{Tu}=1.5 \%$; Data at $\mathrm{Re}_{\mathrm{D}}$ as shown, $\mathrm{Tu}=1.5 \%$

Figure D.14: Seven-Hole Pressure Probe (7HP1) Results: Calibration at $\operatorname{Re}_{\mathrm{D}}=2500, \mathrm{~T} u=1.5 \%$; Data at $\mathrm{Re}_{\mathrm{D}}$ as shown, $\mathrm{Tu}=1.5 \%$ 172

Figure D.15: Seven-Hole Pressure Probe (7HP1) Results: Calibration at $\operatorname{Re}_{\mathrm{D}}=3400, \mathrm{Tu}=1.5 \%$; Data at $\mathrm{Re}_{\mathrm{D}}$ as shown, $\mathrm{Tu}=1.5 \%$

Figure D.16: Seven-Hole Pressure Probe (7HP1) Results: Calibration at $\operatorname{Re}_{\mathrm{D}}=4000, \mathrm{Tu}=1.5 \%$; Data at $\mathrm{Re}_{\mathrm{D}}$ as shown, $\mathrm{Tu}=1.5 \%$

Figure D.17: Seven-Hole Pressure Probe (7HP1) Results: Calibration at $\operatorname{Re}_{\mathrm{D}}=4400, \mathrm{Tu}=1.5 \%$; Data at $\mathrm{Re}_{\mathrm{D}}$ as shown, $\mathrm{Tu}=1.5 \%$

Figure D.18: Seven-Hole Pressure Probe (7HP1) Results: Calibration at $\operatorname{Re}_{\mathrm{D}}=5150, \mathrm{Tu}=1.5 \%$; Data at $\operatorname{Re}_{\mathrm{D}}$ as shown, $\mathrm{Tu}=1.5 \%$ 174 
Figure D.19: Seven-Hole Pressure Probe (7HP1) Results: Calibration at $\operatorname{Re}_{D}=1950, T u=12 \%$; Data at $\operatorname{Re}_{D}$ as shown, $\mathrm{Tu}=12 \%$

Figure D.20: Seven-Hole Pressure Probe (7HP1) Results: Calibration at $\operatorname{Re}_{\mathrm{D}}=2450, \mathrm{Tu}=12 \%$; Data at $\mathrm{Re}_{\mathrm{D}}$ as shown, $\mathrm{Tu}=12 \%$ 175

Figure D.21: Seven-Hole Pressure Probe (7HP1) Results: Calibration at $\operatorname{Re}_{D}=2850, T u=12 \%$; Data at $\operatorname{Re}_{D}$ as shown, $\mathrm{Tu}=12 \%$ 176

Figure D.22: Seven-Hole Pressure Probe (7HP1) Results: Calibration at $\operatorname{Re}_{\mathrm{D}}=3400, \mathrm{Tu}=12 \%$; Data at $\mathrm{Re}_{\mathrm{D}}$ as shown, $\mathrm{Tu}=12 \%$ 176

Figure D.23: Seven-Hole Pressure Probe (7HP1) Results: Calibration at $\mathrm{Re}_{\mathrm{D}}=3800, \mathrm{Tu}=12 \%$; Data at $\mathrm{Re}_{\mathrm{D}}$ as shown, $\mathrm{Tu}=12 \%$

Figure D.24: Seven-Hole Pressure Probe (7HP1) Results for Total Pressure:

Calibration at $\operatorname{Re}_{\mathrm{D}}=2000, \mathrm{Tu}=1.5 \%$; Data at $\mathrm{Re}_{\mathrm{D}}$ as shown, $\mathrm{Tu}=1.5 \% \ldots 178$

Figure D.25: Seven-Hole Pressure Probe (7HP1) Results for Total Pressure:

Calibration at $\operatorname{Re}_{\mathrm{D}}=3400, \mathrm{Tu}=1.5 \%$; Data at $\mathrm{Re}_{\mathrm{D}}$ as shown, $\mathrm{Tu}=1.5 \% \ldots 178$

Figure D.26: Seven-Hole Pressure Probe (7HP1) Results for Total Pressure:

Calibration at $\operatorname{Re}_{\mathrm{D}}=5150, \mathrm{Tu}=1.5 \%$; Data at $\mathrm{Re}_{\mathrm{D}}$ as shown, $\mathrm{Tu}=1.5 \% \ldots . .179$

Figure D.27: Seven-Hole Pressure Probe (7HP1) Results for Dynamic Pressure:

Calibration at $\operatorname{Re}_{\mathrm{D}}=2000, \mathrm{Tu}=1.5 \%$; Data at $\mathrm{Re}_{\mathrm{D}}$ as shown, $\mathrm{T} u=1.5 \% \ldots .179$

Figure D.28: Seven-Hole Pressure Probe (7HP1) Results for Dynamic Pressure:

Calibration at $\operatorname{Re}_{\mathrm{D}}=3400, \mathrm{Tu}=1.5 \%$; Data at $\mathrm{Re}_{\mathrm{D}}$ as shown, $\mathrm{Tu}=1.5 \% \ldots .180$

Figure D.29: Seven-Hole Pressure Probe (7HP1) Results for Dynamic Pressure:

Calibration at $\operatorname{Re}_{D}=5150, T u=1.5 \%$; Data at $\operatorname{Re}_{\mathrm{D}}$ as shown, $\mathrm{Tu}=1.5 \% \ldots .180$

Figure D.30: Seven-Hole Pressure Probe (7HP2) Results: Calibration at $\operatorname{Re}_{\mathrm{D}}=3200, \mathrm{Tu}=1.5 \%$; Data at $\mathrm{Re}_{\mathrm{D}}=3200, \mathrm{Tu}$ as shown 181 
Figure D.31: Seven-Hole Pressure Probe (7HP2) Results: Calibration at $\mathrm{Re}_{\mathrm{D}}=4700, \mathrm{Tu}=1.5 \%$; Data at $\mathrm{Re}_{\mathrm{D}}=4700, \mathrm{Tu}$ as shown

Figure D.32: Seven-Hole Pressure Probe (7HP2) Results: Calibration at $\operatorname{Re}_{\mathrm{D}}=3200, \mathrm{Tu}=12 \%$; Data at $\mathrm{Re}_{\mathrm{D}}=3200$, $\mathrm{Tu}$ as shown

Figure D.33: Seven-Hole Pressure Probe (7HP2) Results: Calibration at $\operatorname{Re}_{D}=4700, T u=12 \%$; Data at $\operatorname{Re}_{D}=4700$, Tu as shown

Figure D.34: Seven-Hole Pressure Probe (7HP2) Results: Calibration at $\operatorname{Re}_{\mathrm{D}}=2350, \mathrm{Tu}=1.5 \%$; Data at $\mathrm{Re}_{\mathrm{D}}$ as shown, $\mathrm{Tu}=1.5 \%$

Figure D.35: Seven-Hole Pressure Probe (7HP2) Results: Calibration at $\operatorname{Re}_{\mathrm{D}}=3200, \mathrm{Tu}=1.5 \%$; Data at $\mathrm{Re}_{\mathrm{D}}$ as shown, $\mathrm{Tu}=1.5 \%$

Figure D.36: Seven-Hole Pressure Probe (7HP2) Results: Calibration at $\operatorname{Re}_{\mathrm{D}}=4700, \mathrm{Tu}=1.5 \%$; Data at $\mathrm{Re}_{\mathrm{D}}$ as shown, $\mathrm{Tu}=1.5 \%$

Figure D.37: Seven-Hole Pressure Probe (7HP2) Results: Calibration at $\mathrm{Re}_{\mathrm{D}}=6400, \mathrm{Tu}=1.5 \%$; Data at $\mathrm{Re}_{\mathrm{D}}$ as shown, $\mathrm{Tu}=1.5 \%$ 184

Figure D.38: Seven-Hole Pressure Probe (7HP2) Results: Calibration at $\mathrm{Re}_{\mathrm{D}}=2350, \mathrm{Tu}=12 \%$; Data at $\mathrm{Re}_{\mathrm{D}}$ as shown, $\mathrm{Tu}=12 \%$

Figure D.39: Seven-Hole Pressure Probe (7HP2) Results: Calibration at $\operatorname{Re}_{\mathrm{D}}=4700, \mathrm{Tu}=12 \%$; Data at $\operatorname{Re}_{\mathrm{D}}$ as shown, $\mathrm{Tu}=12 \%$ 


\title{
Nomenclature
}

\author{
Inferred A value found indirectly from experimental data through the use of a \\ calibration, such as the values of total pressure, dynamic pressure, and \\ flow angle obtained from a multi-hole probe \\ Measured A value found directly from experimental data, such as the pressure \\ sensed at a static tap \\ True The actual value of a property \\ $C_{P o C a l}, C_{P s C a l}, \quad$ Calibration apparatus total, static, and dynamic pressure coefficients \\ $C_{q C a l}$ \\ (Section 3.2, page 35) \\ $C_{\text {Po Krel }}$ \\ Kiel total pressure coefficient (Section 4.3, page 71) \\ $C_{P o W T}$ \\ Wind tunnel total pressure coefficient (Section 4.6, page 129) \\ $C_{\alpha}, C_{\beta}, C_{q} 7 h p$ \\ Seven-hole pressure probe calibration coefficients (Section 3.4.4, \\ $C_{P o} 7 h p$ \\ page 55) \\ $D$ \\ Probe tip outer diameter (Section 2.2.1, page 6) \\ $d$ \\ Probe tip inner diameter (Section 2.2.1, page 6) \\ $K_{\phi 2}, K_{\phi 3}, K_{I 2}$ \\ $K_{13}, K_{1}$ \\ Three-hole pressure probe calibration coefficients (Section 3.4.3, \\ page 49) \\ $L$ \\ Length between the pressure port and probe stem on a static pressure \\ probe (Section 2.2.1, page 6)
}


$L_{o} \quad$ Length between the pressure port and probe tip on a static pressure probe (Section 2.2.1, page 6)

$M$

$P$

$P_{c 1}, P_{c 2}$

$P_{t}$

$P_{o}$

$q$

$R e_{x}$

$T F$

$T u$

$T u_{u}$

$T u_{v}, T u_{w}$

$U, V, W$

$u^{\prime}, v^{\prime}, w^{\prime}$

$\alpha, \beta$

$\triangle C_{P o}$

$\Delta C_{P o K e l}$

$\triangle C_{P o} W T$
Mach number

Static pressure

Reference total and static pressure (Section 3.2, page 34)

Pressure measured by port $i$ of a multi-hole pressure probe

Total pressure

Dynamic pressure

Reynolds number with $\mathrm{x}$ as the characteristic length (Section 2.2.2, page 7)

Turbulence Factor (Section 4.6, page 130)

Resultant turbulence intensity (Section 4.2, page 66)

Turbulence intensity in the streamwise direction (Section 4.2, page 65)

Turbulence intensity in the plane normal to the streamwise axis (Section 4.2, page 65)

Mean components of velocity in the $\mathrm{x}, \mathrm{y}$, and $\mathrm{z}$ directions, respectively

Fluctuating components of velocity in the $\mathrm{x}, \mathrm{y}$, and $\mathrm{z}$ directions, respectively

Probe tangential coordinate system flow angles (Section 3.2, page 37)

Total pressure coefficient difference (Section 4.4.1, page 77)

Kiel total pressure coefficient difference (Section 4.3, page 72)

Wind tunnel total pressure coefficient difference (Section 4.6, page 130) 
$\Lambda_{x}$

$\mu$

$\rho$

$\phi, \theta$
Integral length scale of turbulence in the streamwise direction

Dynamic viscosity

Density

Probe polar coordinate system flow angles (Section 3.2, page 37) 


\section{Chapter 1}

\section{Introduction}

\subsection{Motivation}

Seven-hole pressure probes have been used extensively at Carleton University to make detailed flow field measurements in turbine cascades in both low- and high-speed wind tunnels. Recently, MacIsaac et al. (2009) presented results for total pressure collected using both Kiel and seven-hole pressure probes in the highly three-dimensional and turbulent endwall flow in a low-speed linear turbine cascade. While in agreement throughout most of the flow field, the authors found that the seven-hole probe underestimated total pressure relative to the Kiel probe in some regions. These differences, which were greater than the estimated uncertainties in the measurements, occurred in areas with high turbulence intensities and large gradients in turbulence intensity. Given these results, MacIsaac et al. concluded that turbulence intensity may affect the results obtained from one or both of the probes.

A review of the available literature shows that few studies have investigated the effects of turbulence on the aerodynamic response of multi-hole pressure probes. 
Additionally, while several researchers have investigated the effects of turbulence intensity on aligned Kiel probe measurements, no studies were found regarding Kiel measurements in misaligned flows. Thus, the motivation for the present study was to investigate whether turbulence intensity affects the response of these probes and whether these effects were the cause of the differences noted by MacIsaac et al.

\subsection{Thesis Approach and Objectives}

The present author has modified an existing low-turbulence probe calibration rig to generate a calibration jet at a user-controlled turbulence intensity of up to $12 \%$. This rig was then used to collect Kiel, three-hole, and seven-hole probe data at several turbulence intensities and Reynolds numbers. Although the primary motivation for the present study was the influence of turbulence intensity, Reynolds number was also investigated because a review of the available literature found few studies have examined these probes at Reynolds numbers in the range of 1000 to 5000 . This range is typically experienced in the measurements from the Carleton University low-speed cascade wind tunnel.

The objective of the Kiel probe measurements was to determine if the probe's sensitivity to flow angle changed at different operating points. As documented by Chue (1975), total pressure measurements from Kiel probes in laminar flows are known to be insensitive to flow angles of less than approximately $35^{\circ}$; the present author, however, found no research documenting whether this insensitivity changes in turbulent flows with varying turbulence intensities. Thus, measurements have been collected using the Kiel 
probe over flow angles of 0 to $50^{\circ}$ at Reynolds numbers of 1050 to 4350 and turbulence intensities of 1.5 to $12 \%$ to determine if either flow parameter affects the aerodynamic behaviour of the probe.

For the three- and seven-hole probes, the objective was to determine if their general response varied in flows of different Reynolds numbers and turbulence intensities. To meet this objective, the probes were calibrated at several operating points and then used to reduce data collected in a known flow at the same and different operating points. By comparing the results to the true conditions in the flow, any changes in probe behaviour were identified. In particular, this approach quantified any systematic errors caused by calibrating the probe at one operating point and then reducing data at another. Although the motivation for this study originated from the measurement of total pressure using a seven-hole probe, the effects on flow angle, total pressure, and dynamic pressure using both three- and seven-hole probes were investigated. These additional studies provide a better understanding of general multi-hole probe response.

The final objective of this thesis was the development of a data reduction procedure that accounts for the influence of both Reynolds number and turbulence intensity on multi-hole probes simultaneously. This new reduction scheme is demonstrated by applying it to the seven-hole probe data collected by MacIsaac et al. (2009). 


\subsection{Overview of Thesis}

Chapter 2 provides a review of the previous literature relevant to the present thesis. This review includes a summary of the factors known to affect the aerodynamic response of single- and multi-hole pressure probes and the available literature on turbulence generation techniques. The following chapter provides a description of the experimental apparatus, instrumentation, and data reduction techniques used in this thesis. Chapter 4 presents the work conducted for the present study including the modifications to the calibration rig and the data collected using the Kiel, three-hole, and seven-hole pressure probes. Also included in Chapter 4 is the examination of the seven-hole probe data from MacIsaac et al. (2009) using a new data reduction technique that accounts for the influence of Reynolds number and turbulence intensity on multi-hole probe behaviour. Finally, Chapter 5 summarizes the conclusions from the present study and provides recommendations for future work. 


\section{Chapter 2}

\section{Literature Review}

\subsection{Introduction}

This chapter summarizes the available literature on the effects of some flow field characteristics, such as Reynolds number and turbulence intensity, on measurements made by single- and multi-hole pressure probes. The current work focuses on the effects of Reynolds number and turbulence intensity specifically, but this chapter also summarizes other flow characteristics that could influence measurements.

Finally, the available literature for turbulence generation is summarized. Although this literature guided the development of the High-Turbulence Probe Calibration Rig, the rig used in the present study is believed to be of a novel design and notably different from those in the open literature. This is discussed further in Section 4.2. 


\subsection{Factors Influencing Single-Hole Pressure Probe Flow Measurements}

\subsubsection{Introduction}

Single-hole probes are defined here as probes with a single or several connected pressure ports. Many single-hole pressure probe designs exist. Some examples are shown in Figure 2.1.

Figure 2.1a shows a subsonic static pressure probe. The pressure port is located along the probe body such that the measured pressure is essentially the undisturbed flow static pressure. Design guidelines for static pressure probes are available in Chue (1975). Pressure port size and shape, length from the probe tip to the port $\left(L_{o}\right)$, length from the port to the probe stem $(L)$, and tip shape are common design parameters for these probes.

The Pitot probe, shown in Figure 2.1b, measures total pressure by stagnating the flow at the probe's forward-facing pressure port. Chue (1975) presents guidelines for probe
a)
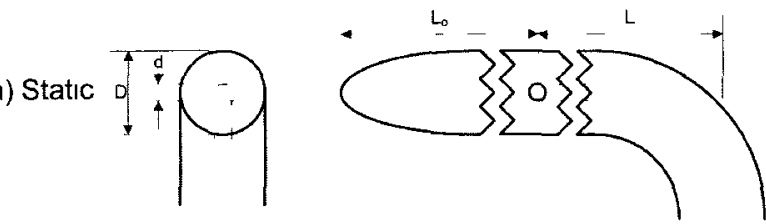

b) Pitot
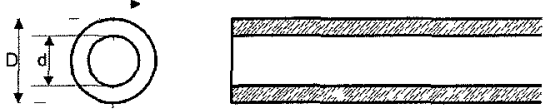

c) Kiel
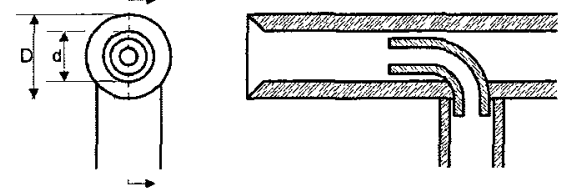

Figure 2.1: Single-Hole Pressure Probes 
The Pitot probe, shown in Figure $2.1 \mathrm{~b}$, measures total pressure by stagnating the flow at the probe's forward-facing pressure port. Chue (1975) presents guidelines for probe design parameters including tip shape, probe diameter, and wall thickness. The Kiel probe, shown in Figure 2.1c, is a variation on the Pitot probe designed to be less sensitive to flow misalignment. The sheath around the Kiel probe pressure port aligns incoming flow with the port. Kiel probes are commonly used for total pressure measurement in flows with unknown or varying flow angles.

Described below is the available literature on how Reynolds number, flow misalignment, Mach number, velocity gradients, turbulence and unsteadiness, and wall proximity influence the aerodynamic response of these probes.

\subsubsection{Influence of Reynolds Number}

Reynolds number is the ratio of inertial to viscous forces defined by:

$$
\mathrm{Re}_{x}=\frac{\rho V x}{\mu}
$$

where $\rho$ is the fluid density, $\mu$ the fluid dynamic viscosity, $V$ the magnitude of velocity, and $x$ the characteristic length. Reynolds number sensitivity of Pitot probes is documented by MacMillan (1954a, b) as shown in Figure 2.2. 

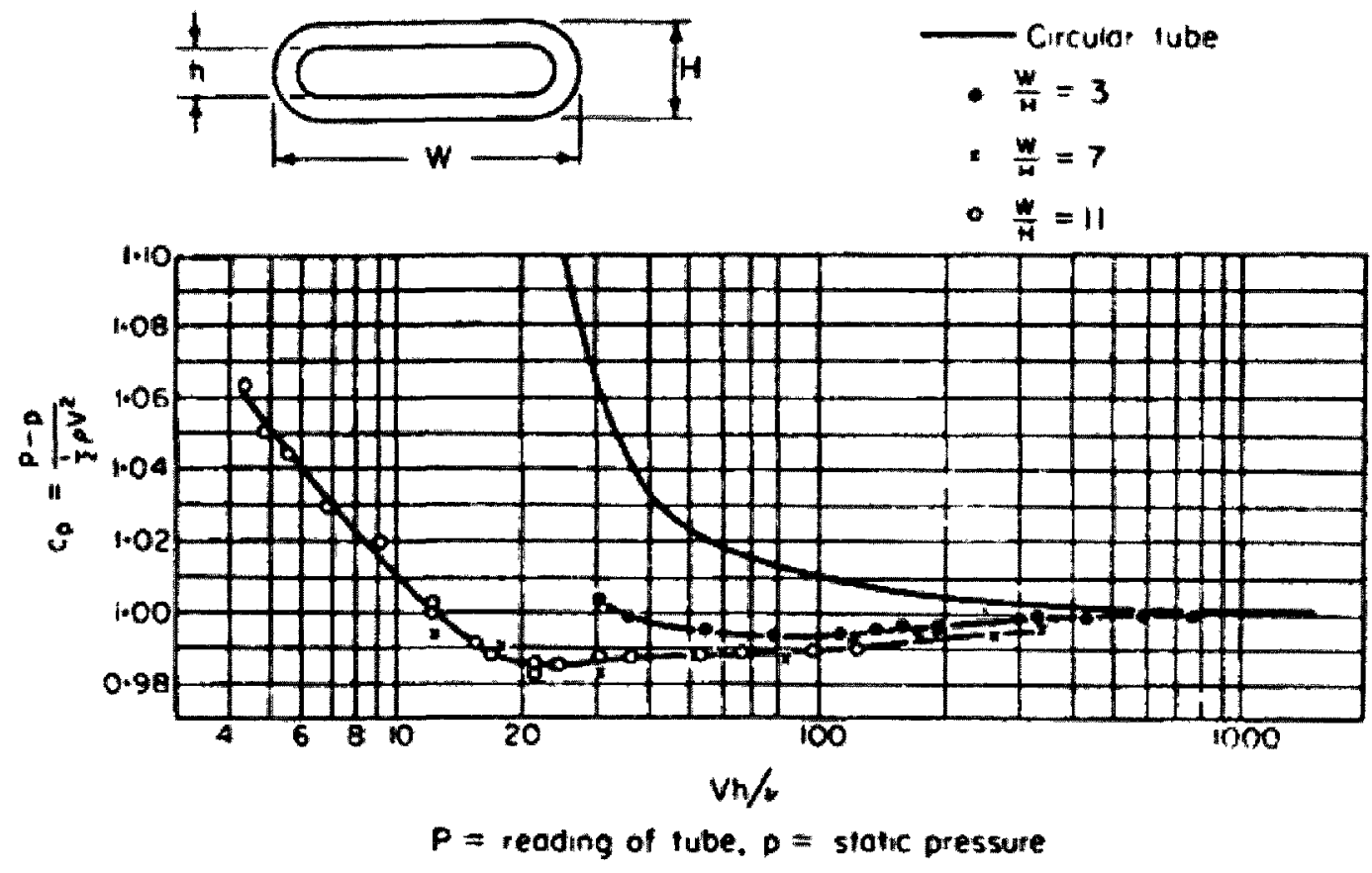

Figure 2.2: Pitot Tube Reynolds Number Effects, from MacMillan (1954)

MacMillan found that Pitot probes are insensitive to Reynolds numbers greater than about 600 when Reynolds number is defined using the height of the probe tip, $H$, as shown in the figure. At lower Reynolds numbers, circular mouthed Pitot probes were found to overestimate total pressure. Flat mouthed probes over or underestimated total pressure at low Reynolds numbers as shown in Figure 2.2. Chue (1975) presents similar results for other total pressure probe geometries.

Chue cites Schulze et al. (1952) stating that static pressure probes are insensitive to Reynolds number for Reynolds numbers between 3000 and 53000 but that probe response is likely dependent on Reynolds number for $\operatorname{Re}_{D}<3000$. Chue does not provide additional information on static probe behaviour at low Reynolds numbers. 


\subsubsection{Influence of Flow Misalignment}

The aerodynamic behaviour of static pressure probes is known to be influenced by flow misalignment. Krause and Gettelman (1952), as summarized by Chue (1975), found the results in Figure 2.3. These results, presented as the error in the measured static pressure normalized by dynamic pressure, show the probe underestimates static pressure when misaligned with the flow. Cho and Becker (1985) present additional results for the Prandtl probe that are in agreement with Krause and Gettelman.

Figure 2.4 shows results from Chue for the influence of flow misalignment on the measurement of total pressure. Included in the figure are a square profile Pitot probe (1), a hemispherical profile Pitot probe (4), a Kiel probe (5), and others. The critical angle for each probe, defined as the angle at which the error in total pressure is equal to $1 \%$ of dynamic pressure, is quoted. Rossow (1991) presents similar results. As seen in Figure 2.4, the Kiel probe is insensitive to flow misalignment up to about $35^{\circ}$. Chue,

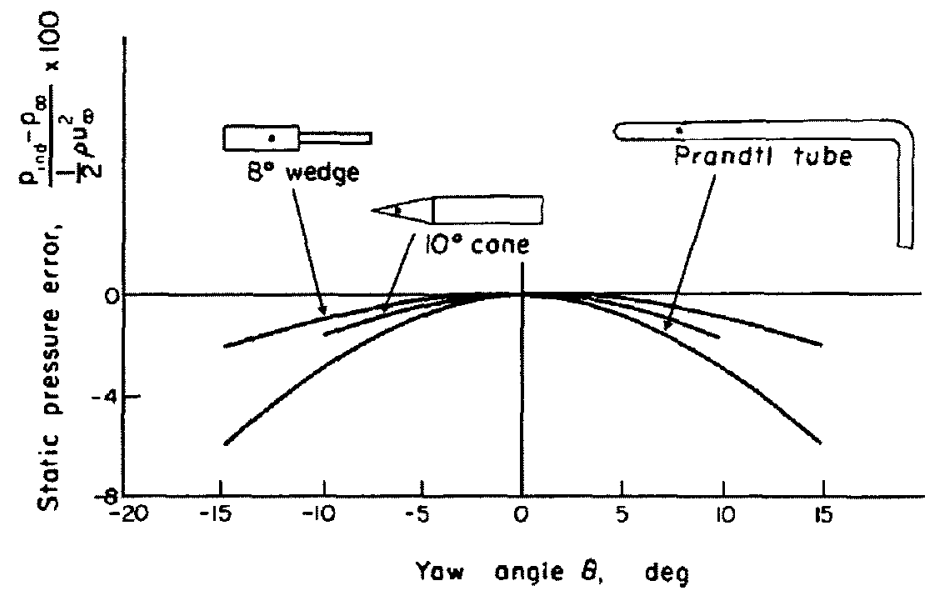

Figure 2.3: Static Pressure Probe Flow Misalignment Effects, from Krause and Gettelman (1952) 
summarizing Walchner (1939) and Gracey et al. (1951), states the critical angles for the Pitot and Kiel probes decrease when $M>0.5$. Ower and Pankhurst (1977) show in Figure 2.5 that the critical angles for hemispherical Pitot probes are also affected by the ratio of inner and outer tip diameter. No literature was found on the influence of turbulence intensity on critical angle; Section 4.3 of the present text discusses this topic in more detail for the Kiel probe.

Becker and Brown (1974) suggest that Pitot probe response to flow misalignment in laminar flows can be modelled as:

$$
P_{s}-P=\frac{1}{2} \rho U^{2}\left[1-K\left(\sin ^{2} \theta\right)^{m}\right]
$$

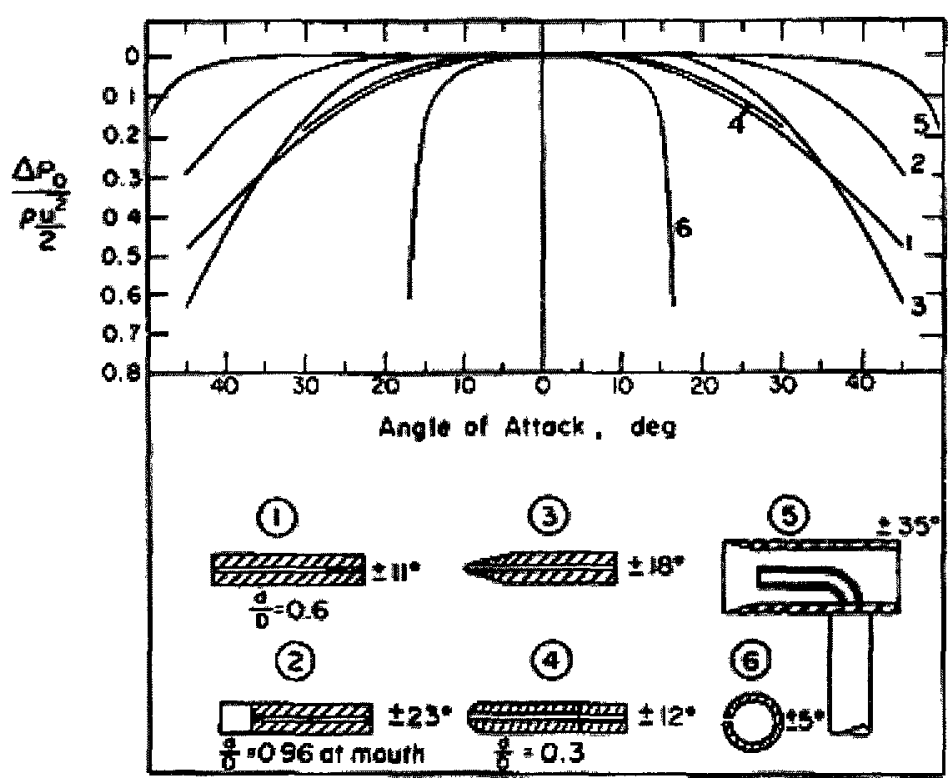

Figure 2.4: Total Pressure Probe Flow Misalignment Effects, from Chue (1975) 


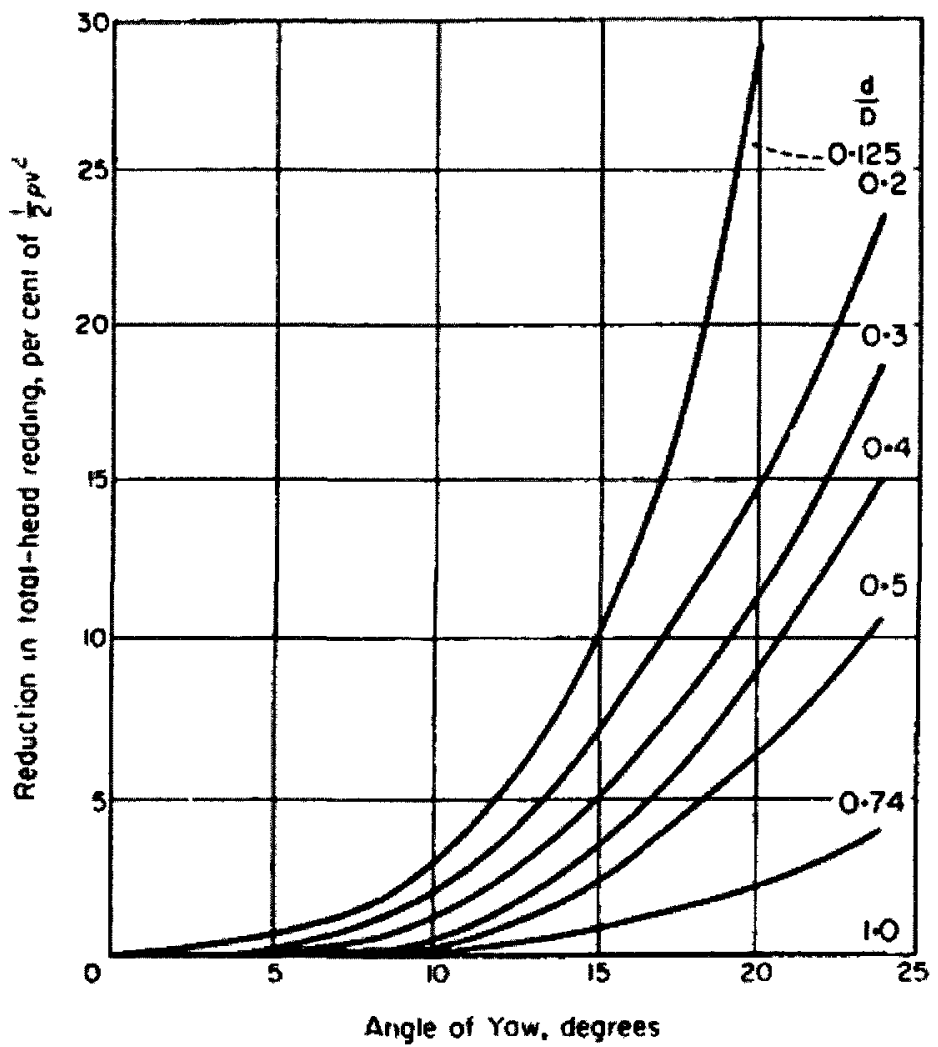

Figure 2.5: Hemispherical Pitot Probe Flow Misalignment Effects with Various Tip Diameter Ratios, from Ower and Pankhurst (1977)

where $P_{s}$ is the pressure sensed by the probe, $P$ is the true static pressure, and $K$ and $m$ are constants that depend on $d / D$ and tip shape. The authors found the values of $K$ and $m$ for various probes experimentally by measuring total pressure in a known flow at different flow angles $(\theta)$. Typical values of $K$ were 1 to 5 . Typical values of $m$ were 1.1 to 3. Experimental validation by Becker and Brown showed Equation 2.2 to be accurate up to $60^{\circ}$ misalignment. 


\subsubsection{Influence of Mach Number}

Chue (1975) describes the effect of Mach number, or compressibility, on single-hole pressure probes. For the measurement of total pressure in subsonic flows, Mach number has a negligible influence on results in aligned flows. Mach numbers greater than about 0.5 however do result in a decrease in the critical angle for Pitot and Kiel probes. Chue also states that total pressure probes underestimate total pressure in supersonic flows due to the presence of a shock upstream of the probe. True total pressure can however be obtained from these supersonic measurements through the use of the Rayleigh Pitot formula, as described in more detail by Chue.

For Mach numbers greater than about 0.5 , the accuracy of static pressure measurements is dependent on Mach number and probe geometry. The optimal probe geometry varies with Mach number and thus high accuracy requires a probe designed for a specific Mach number. Ower and Pankhurst (1977) and Sieverding (1975) provide guidelines for probe design, such as tip shape and hole location, at various Mach numbers. Chue summarizes additional probe designs and presents data showing that static pressure measurement is unaffected by Mach number when Mach number is less than 0.5 .

It should be noted that the present work investigates only incompressible flows of Mach numbers less than 0.15 . Mach number should not influence the results presented here. 


\subsubsection{Influence of Velocity Gradient}

Velocity gradients can significantly influence measurements by pressure probes. The most dominant effect of velocity gradients on total pressure measurement is the deflection of the streamlines near the probe. A schematic example of this is shown in Figure 2.6. Originally documented by Young and Mass (1936) and since investigated by Tavoularis and Szymczak (1989) and others, this downwash causes the probe to measure total pressure along a streamline offset by a distance $\delta$ from the probe axis. The ratio of this deflection to the probe outer diameter, $\delta / D$, is typically presented relative to the shear parameter, $K$, expressed as:

$$
K=\frac{D}{2 V} \frac{d V}{d y}
$$

where $V$ is the mean flow velocity. For flows where $K$ is greater than 0.03 , Tavoularis and Szymczak show that $\delta / D$ depends solely on probe geometry $(d / D$ and tip shape) and is constant for all values of shear parameter. Typical values of $\delta / D$ are in the order of 0.18 . For flows where $K$ is less than 0.03 , the effects of velocity gradients are negligible.

Static pressure probes also experience a downwash effect like that for Pitot probes. Chue (1975) suggests the effect of velocity gradients on static pressure measurement is similar to that of small flow misalignments, citing unpublished works for validation. 


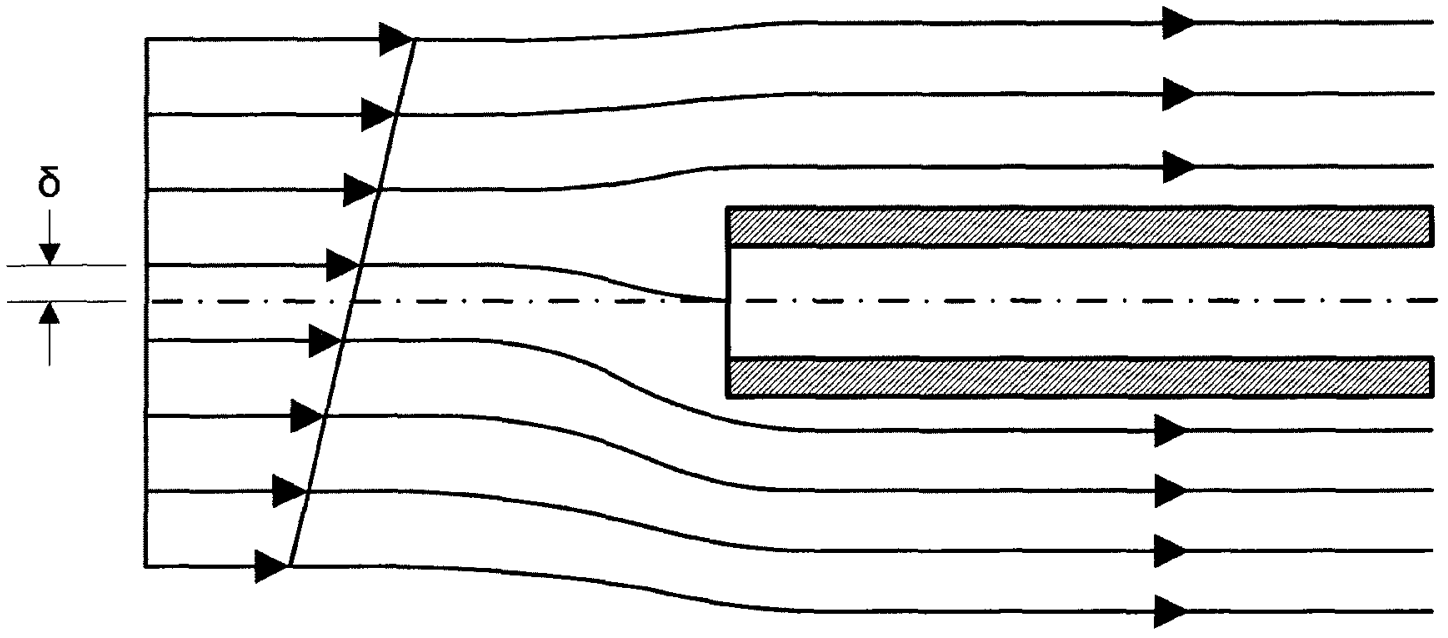

Figure 2.6: Pitot Probe in a Velocity Gradient

It should be noted that the present work investigates flows with minimal velocity gradients. Typical values for shear parameter in the current study are less than 0.01 . Thus, the effects of velocity gradients on results discussed here are expected to be negligible.

\subsubsection{Influence of Turbulence and Unsteadiness}

Figure 2.7 shows results from Christiansen and Bradshaw (1981) for static pressure measurements in laminar and turbulent flows. Data were collected using a "standard" static pressure probe like that shown in Figure 2.1a. Results are presented using $C_{p}$ defined as:

$$
C_{p}=\frac{P_{m}-P_{a}}{P-P_{a}}
$$


where $P_{m}$ is the measured static pressure, $P$ is the true static pressure, and $P_{a}$ is ambient pressure. $C_{p}$ is shown in the figure as a function of flow misalignment, $\psi$. The response in laminar flow is indicated by circles and in turbulent flow by triangles. Christiansen and Bradshaw found that turbulence effects caused an underestimation of static pressure for aligned static pressure probes and a reduced sensitivity to flow misalignment.

Christiansen and Bradshaw (1981) and Cho and Becker (1985) investigated the influence of the integral length scale of turbulence $(\Lambda)$ on measurements using a static pressure probe. Both studies found that the effect turbulence has on measurements increased as $D / \Lambda$ increased. Cho and Becker state this effect is caused by the interaction between the turbulent eddies and the probe body upstream of the pressure port; they found the effect increased with increasing distance between the probe tip and pressure

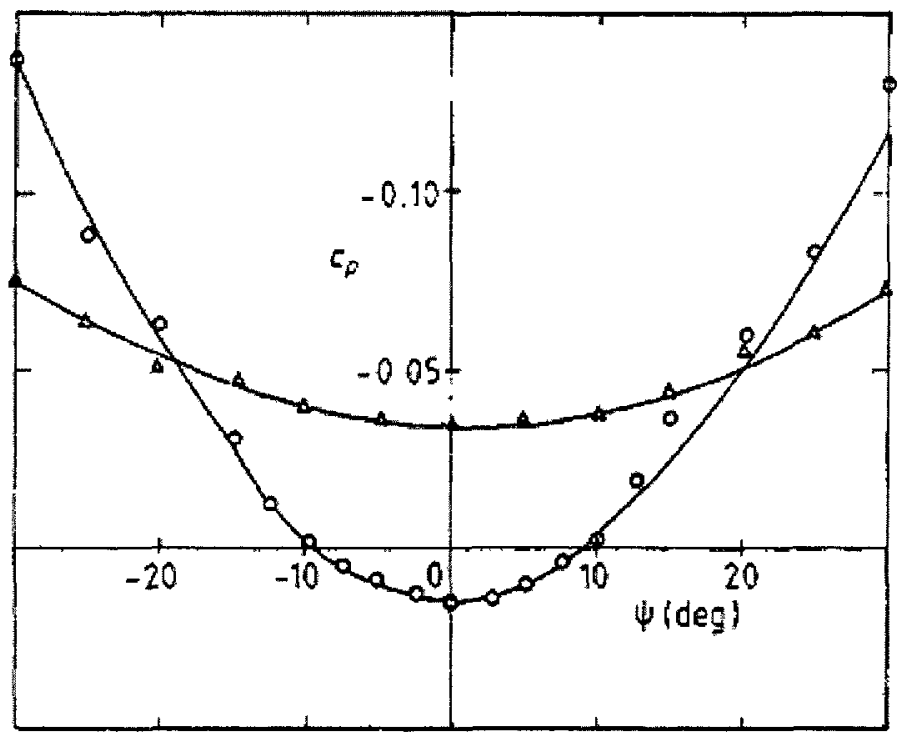

Figure 2.7: "Standard" Static Pressure Probe Flow Misalignment Effects in Turbulent Flow, from Christiansen and Bradshaw (1981) 
ports $\left(L_{o}\right.$ in Figure 2.1). Cho and Becker also recommend the use of probes with a single pressure port to prevent any effect due to flow between ports.

Goldstein (1936) first predicted that Pitot probes would overestimate total pressure in turbulent flows according to:

$$
P_{\text {oMeasured }}=P+\frac{1}{2} \rho \overline{\mathrm{V}^{2}}
$$

where:

$$
\overline{\mathrm{V}^{2}}=U^{2}+V^{2}+W^{2}+\overline{u^{\prime 2}}+\overline{v^{\prime 2}}+\overline{w^{\prime 2}}
$$

$U, V$, and $W$ are the mean velocity components in the $\mathrm{x}, \mathrm{y}$, and $\mathrm{z}$ direction and $u^{\prime}, v^{\prime}$, and $w^{\prime}$ are the instantaneous velocity fluctuations. By combining Equations 2.5 and 2.6 we obtain:

$$
P_{\text {oMeasured }}=P+\frac{1}{2} \rho \overline{\mathrm{V}}^{2}+\frac{1}{2} \rho \overline{\mathrm{V}^{\prime 2}}
$$

where:

$$
\overline{V^{\prime 2}}=\frac{\overline{u^{\prime 2}}+\overline{v^{\prime 2}}+\overline{w^{\prime 2}}}{3}
$$

and thus: 


$$
P_{\text {oMeasured }}=P_{\text {oTrue }}+\frac{1}{2} \rho \overline{\mathrm{V}^{\prime 2}}
$$

Equation 2.9 indicates that the measured total pressure is overestimated proportionally to the sum of the mean square of the velocity fluctuations $\overline{\left(\mathrm{V}^{\prime 2}\right)}$. This relates to the turbulence intensity through:

$$
T u=\frac{\sqrt{\overline{V^{\prime 2}}}}{\bar{V}}
$$

Goldstein proposed this relation irrespective of probe geometry.

Both Becker and Brown (1974) and Rossow (1991) numerically examined the influence of probe geometry on the response of total pressure probes in turbulent flows. Becker and Brown used their model for the response of total pressure probes in misaligned flows, discussed in Section 2.3.3, to describe the effect of turbulence on the probe. They found probe geometry, such as tip shape and inner to outer diameter ratio $(d / D)$ significantly influenced probe response in turbulent flows. Building on this work, Rossow investigated the influence of probe tip geometry on total pressure measurement in turbulent flows analytically. He too found that probe geometry was a significant factor in probe response to turbulent flow. Thus, although a reasonable estimate, Equation 2.9 oversimplifies the response of the probe to turbulence.

Chue (1975), describing the work of Walsche and Garner (1960), states that the Kiel probe overestimates the total pressure in turbulent flows less than the standard Pitot 
probe. Kiel probes are less affected by turbulence because they are less sensitive to flow misalignment. Chue also states that very high levels of turbulence can reduce critical angle for Kiel probes but provides no quantitative analysis.

\subsubsection{Influence of Wall Proximity}

Pressure probes can distort or displace streamlines when near a body. This effect causes errors in near wall measurements. Tavoularis (2005) and Blake (1983) suggest that measurements be made at least two outer probe diameters from a wall, although this may be conservative depending on probe type and size.

In the present work, all data is collected well away from walls. Chapter 4 does not consider wall-proximity effects.

\subsection{Factors Influencing Multi-Hole Pressure Probe Flow Measurements}

\subsubsection{Introduction}

Multi-hole pressure probes incorporate two or more independent pressure ports. Apart from Pitot-static probes, multi-hole pressure probes typically require calibration. The pressure sensed at each port is used in calibration coefficients to infer flow properties. Depending on the number and placement of the ports, total pressure, static pressure, and flow angle may be obtained. Three examples of multi-hole probes are presented in Figure 2.8. 
Figure 2.8a shows a three-hole pressure probe. This probe has a central forward-facing port and two chamfered side ports. Total pressure, static pressure, and flow angle in the plane of the pressure ports (designated here as yaw) can be inferred with this probe. Figures $2.8 \mathrm{~b}$ and $2.8 \mathrm{c}$ show five- and seven-hole pressure probes, respectively. These probes have ports in multiple axes and allow for the inference of total pressure, static pressure, and flow angles in pitch and yaw.

Described below is the available literature on how Reynolds number, flow misalignment, Mach number, velocity gradients, turbulence and unsteadiness, and wall proximity influence the aerodynamic response of these probes

\subsubsection{Influence of Reynolds Number}

Benner (2003) examined the effect of low Reynolds numbers on three-hole pressure probe measurements at yaw angles of -10 to $10^{\circ}$. Figure 2.9 presents his results. Figure

a) Three-hole

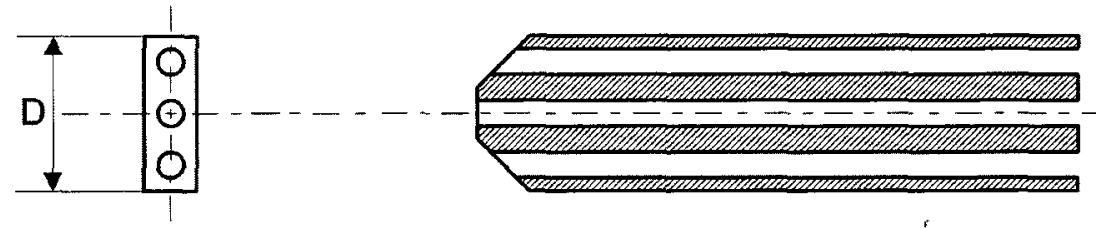

b) Five-hole

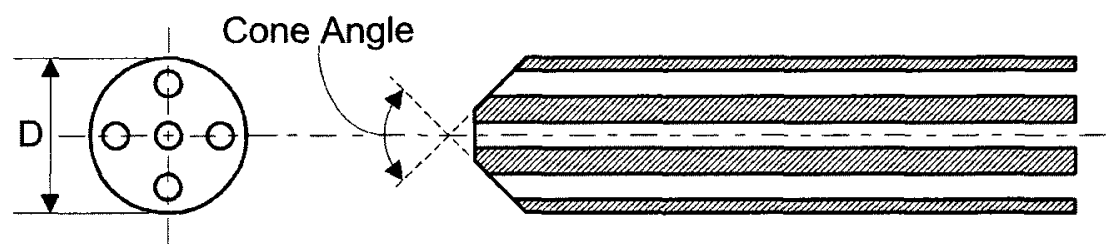

c) Seven-hole

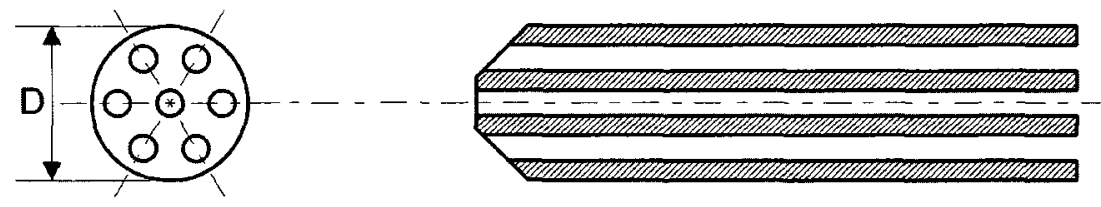

Figure 2.8: Multi-Hole Pressure Probes 
$2.9 \mathrm{a}$ shows the error in flow angle; Figures $2.9 \mathrm{~b}$ and $2.9 \mathrm{c}$ show the errors in dynamic and total pressure normalized by true dynamic pressure. Benner found Reynolds number affected the inferred dynamic pressure for three-hole probes; Reynolds number did not influence the measurement of flow angle or total pressure.

Wenger and Devenport (1999) document the effect of low Reynolds numbers on flow measurement using an aligned seven-hole pressure probe. The authors calibrated their probe at a Reynolds number of 3900 and used this calibration to reduce data taken at a Reynolds number of 2500 . Reducing the lower Reynolds number data using the higher Reynolds number calibration resulted in a $1 \%$ underestimation of the flow velocity. These results are relatively consistent with results from Benner for the three-hole probe. Additional measurements by Wenger and Davenport showed the Reynolds number effects were significant up to Reynolds numbers of at least 8000 .

Figure 2.10 presents the values of the seven-hole probe calibration coefficients for dynamic pressure, $C_{q}$, and total pressure, $C_{o}$, from Maclsaac et al. (2009). These values are shown for five Reynolds numbers over the range of 2500 to 5200 . This figure shows that the calibration coefficient for dynamic pressure varies with Reynolds number for all flow angles. Conversely, the calibration coefficient for total pressure is insensitive to 


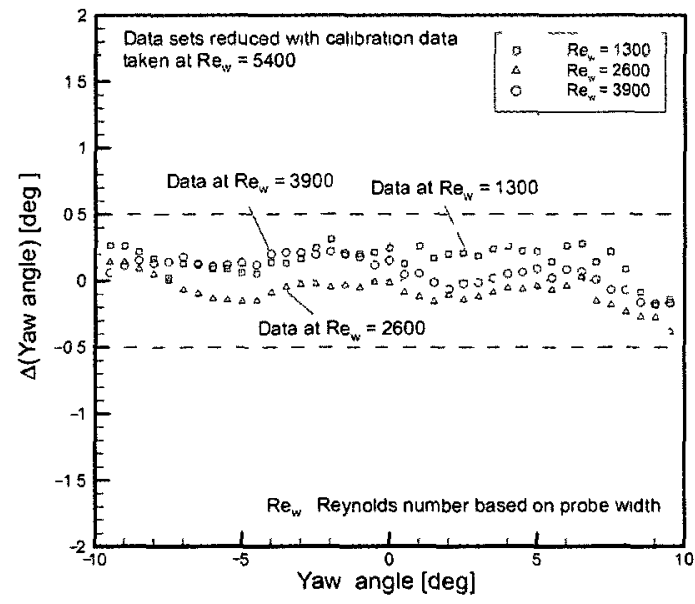

(a)

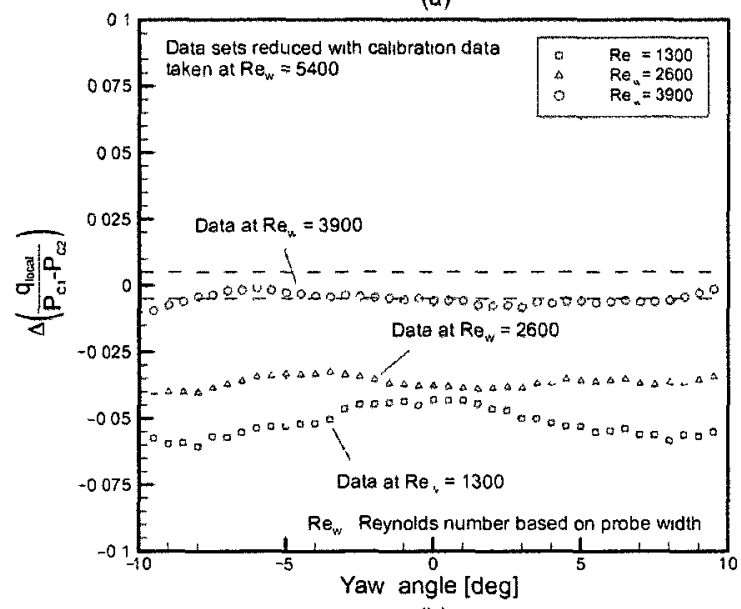

(b)

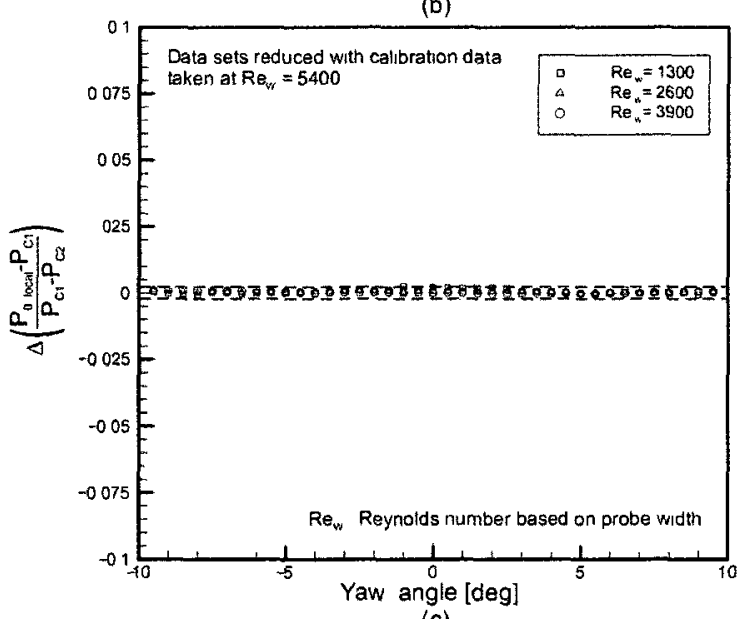

(c)

Figure 2.9: Three-Hole Pressure Probe Reynolds Number Effects, from Benner (2003) 
Reynolds number except at high flow angles. For reducing data collected in flows with varying Reynolds numbers, MacIsaac et al. recommend calibrating the seven-hole probe at several Reynolds numbers and interpolating between these calibrations during data reduction in order to reduce the error caused by Reynolds number effects.

Dominy and Hodson (1993) investigated the effect of Reynolds number on five-hole pressure probes for Reynolds numbers of 10000 to 80000 . Their results are presented
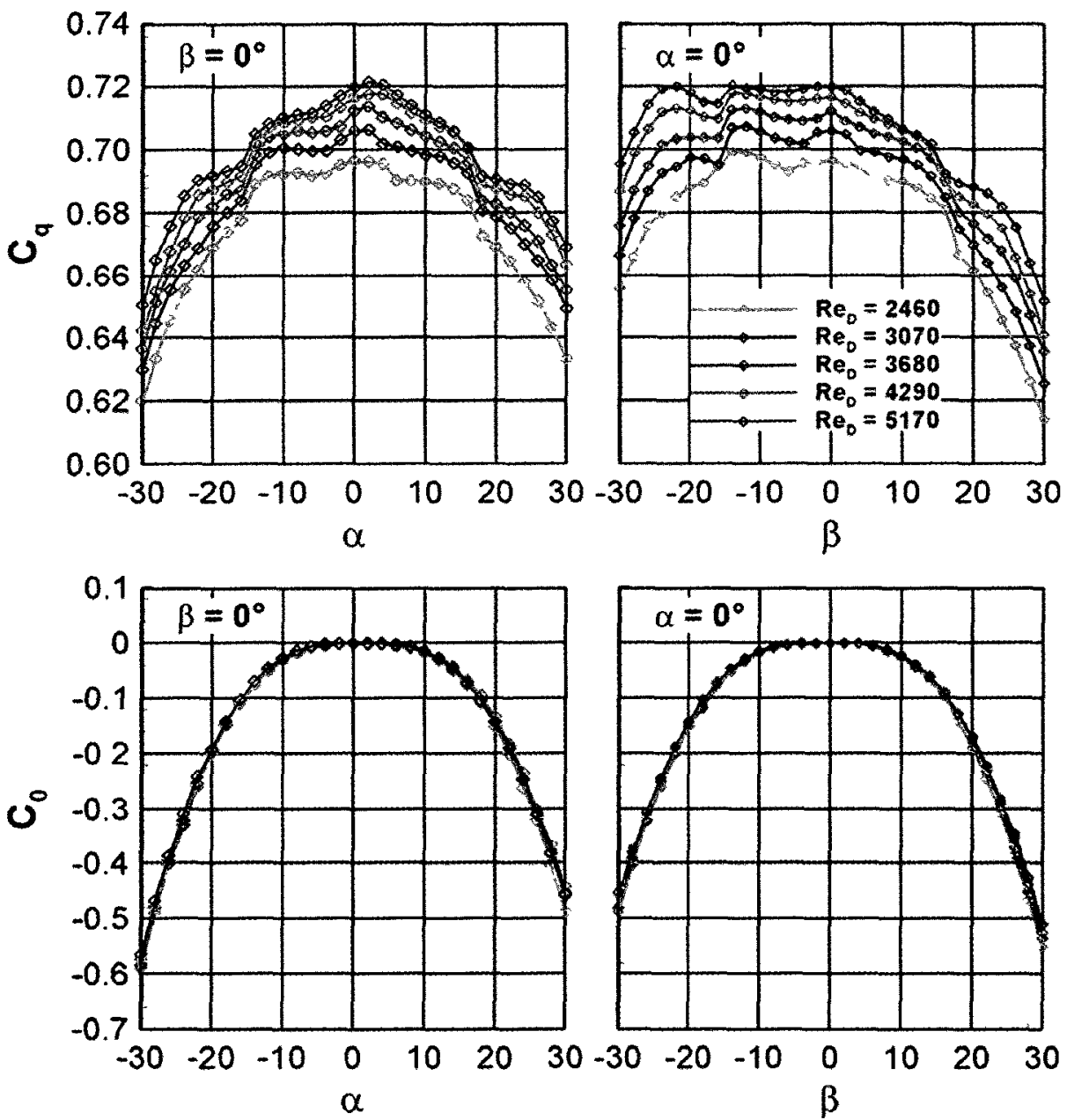

Figure 2.10: Seven-Hole Pressure Probe Reynolds Number Effects, from MacIsaac et al. (2009) 
using the pressure coefficient for each pressure port, defined as:

$$
C_{P_{t}}=\frac{P_{t}-P_{o}}{P_{o}-P}
$$

Where $P_{i}$ is the pressure at the port, $P_{o}$ is total pressure, and $P$ is static pressure. Figure 2.11 shows $C_{p}$ results from Dominy and Hodson for a yaw port with the probe yawed through -20 to $20^{\circ}$ at constant $0^{\circ}$ pitch. Yaw angle is positive when the port is on the windward side of the probe and negative on the leeward side. Figure 2.11a presents contours of $C_{p}$ as a function of yaw angle and Reynolds number. Figure $2.11 \mathrm{~b}$ presents curves of $C_{p}$ at various Reynolds numbers.

Reynolds number effects can be seen in Figure 2.11 for both low and high Reynolds numbers. For Reynolds numbers less than about 21000, Dominy and Hodson note a

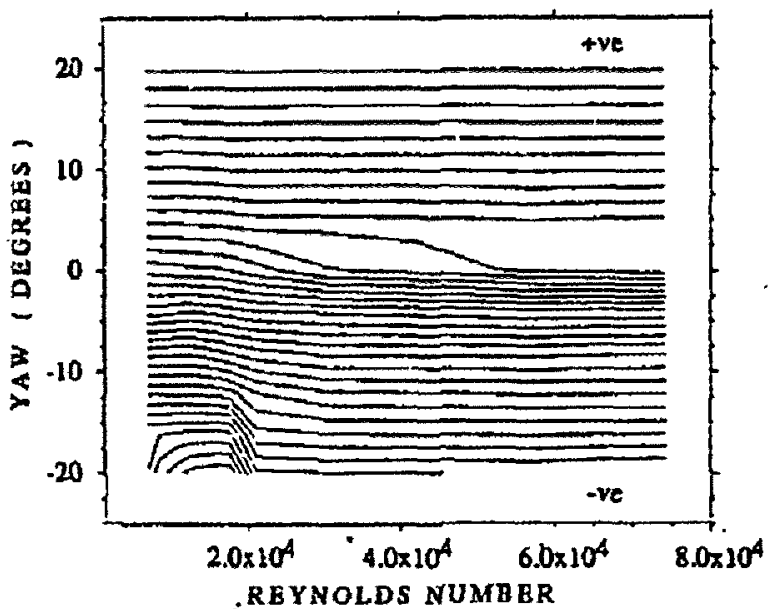

(a)

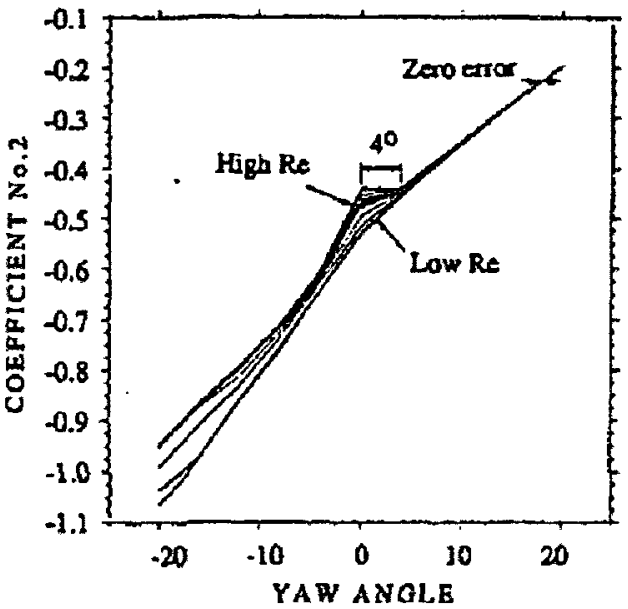

(b)

Figure 2.11: Reynolds Number Effects on a Yaw Hole of a Five-Hole Pressure Probe, from Dominy and Hodson (1993): (a) Contour Interval=0.025; (b) Reynolds Number Interval of $5 \times 10^{3}$ starting at $10 \times 10^{3}$ 
strong Reynolds number influence at large negative yaw angles. This influence causes the yaw hole pressure coefficient to decrease at these Reynolds numbers. Johnson (1985) found that a separation bubble occurs on the leeward side of a five-hole probe at these conditions. Figure 2.12 shows this separation for a probe at a Reynolds number of 13000 and at $8^{\circ}$ yaw, $0^{\circ}$ pitch: the separation originates at the leading edge of the probe and extends over part of the yaw hole before reattaching. Dominy and Hodson concluded that this separation affects the pressure coefficient for the yaw hole and that the influence of Reynolds number on this separation was the mechanism by which Reynolds number affected the yaw calibration at $R e_{D}<21000$. Furthermore, Dominy and Hodson showed that this Reynolds number effect was probe geometry dependent: the Reynolds number at which this effect occurred was lower for probes with larger cone angles, defined in Figure 2.8, because it was comparatively easier for the flow to follow the shape of these probes when inclined with the flow. The effects were also less prominent for probes with forward-facing holes than perpendicular holes, shown in Figure 2.13, for similar reasons.

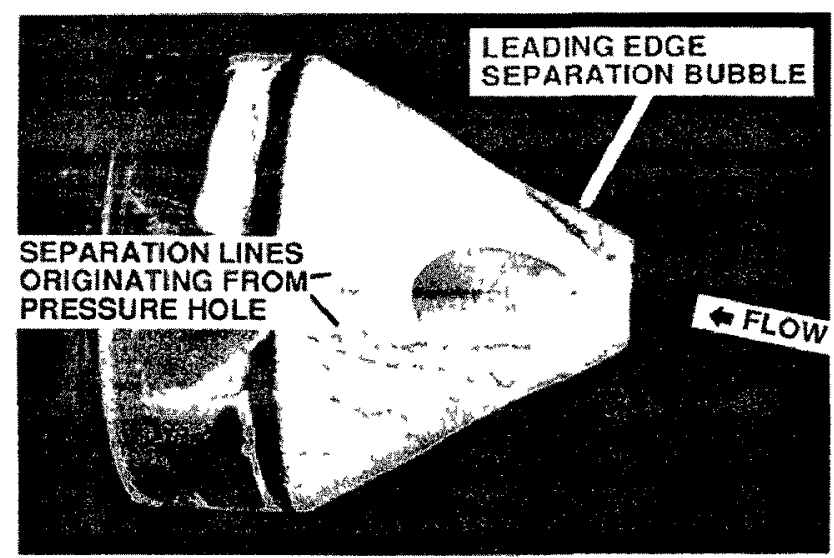

Figure 2.12: Flow Separation on the tip of a Five-Hole Pressure Probe, from Johnson (1985) 

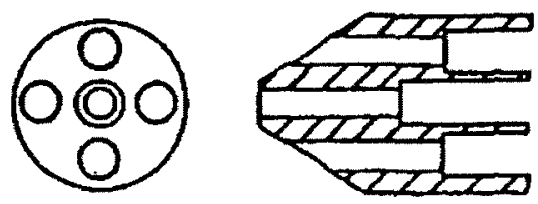

Forward facing cone
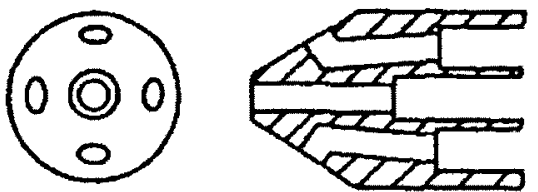

Perpendicular cone

Figure 2.13: Five-hole Probe Geometry, from Dominy and Hodson (1993)

\subsubsection{Influence of Flow Misalignment}

Three-, five-, and seven-hole pressure probes are designed for flow angle measurement. Three-hole probes can measure yaw angles of 20 to $30^{\circ}$ in pitch-aligned flow. Five-hole probes can measure flow angles of 20 to $40^{\circ}$ in both pitch and yaw according to Everett et al. (1983) and others. Flow separation on the leeward side of the probe tip prevents the use of either type of probe beyond these angle ranges.

Seven-hole probes were designed for use in flows with large angle variations. Like with the other multi-hole probes, separation occurs on the leeward side of the seven-hole probe. The difference here is that the redundancy in the seven pressure ports results in at least four ports located in the attached region of the flow even at high flow angles. By using only the ports in the attached region for data reduction, these probes can accurately measure highly angular flows. Gerner et al. (1984) reports accurate flow measurements at up to $70^{\circ}$ misalignment and previously at Carleton University the probes have been calibrated and used for flow angles of up to $50^{\circ}$. The current work investigates multihole probe response for flow angles of less than $35^{\circ}$. 


\subsubsection{Influence of Mach Number}

Gerner et al. (1984) cites Barker et al. (1979) in stating that the seven-hole probe calibration coefficients are affected by compressibility. Zeiger et al. (1998) found sevenhole probe calibration coefficients were a function of Mach number above Mach 0.6. Dominy and Hodson (1993) found five-hole probe calibration is also affected by compressibility. MacIsaac et al. (2009) account for Mach number effects on seven-hole probe measurements by calibrating at several Mach numbers and interpolating between calibrations when reducing data. Measurements in supersonic flows also require shock corrections, similar to Section 2.2.4.

It should be noted that the present work is in the incompressible flow regime $(M<0.3)$ and compressibility effects are expected to be negligible.

\subsubsection{Influence of Velocity Gradient}

Like single-hole probes, multi-hole probes deflect the incoming streamlines as described in Section 2.2.5. When measuring flows with velocity gradients, these probes effectively measure a streamline offset from the probe centre by a distance $\delta . \quad \delta$ is dependent on probe geometry. Multi-hole probes are also affected by the spacing between their ports because all ports measure a slightly different location in the flow. For moderate velocity gradients, minimizing probe size can provide sufficient accuracy. Flow measurements in large gradients however require the use of a correction. 
Based on the work of Ligrani et al. (1989), Chernoray and Hjarne (2008) describe a correction scheme for multi-hole probe measurements in velocity gradients. This correction scheme was shown to have reasonable accuracy through comparison with hotwire measurements. Like Chernoray and Hjarne, Pisterman (2004) also proposes a velocity gradient correction scheme for seven-hole probes and provides experimental validation.

It should be noted that the present work investigates flows with minimal velocity gradients. Probes used here are also less than $2.4 \mathrm{~mm}$ in diameter. Thus, velocity gradients should not influence the results discussed in Chapter 4.

\subsubsection{Influence of Turbulence and Unsteadiness}

Dominy and Hodson (1993) conducted a brief study on the effect of turbulence on five-hole probe response; their results are shown in Figure 2.14 which presents contours of yaw calibration coefficient for a five-hole probe at streamwise turbulence intensities of 0.1 and $10 \%$. They found that turbulence intensity had an effect on five-hole pressure probe yaw coefficient when the probe Reynolds number was less than 20000 . As discussed in Section 2.3.2, Johnson (1985) found a separation bubble on the leeward side of a yawed five-hole probe at a Reynolds number of 13000. Dominy and Hodson suggest that, like with Reynolds number, the mechanism by which turbulence intensity affects the probe yaw coefficient is through influencing this flow separation. 


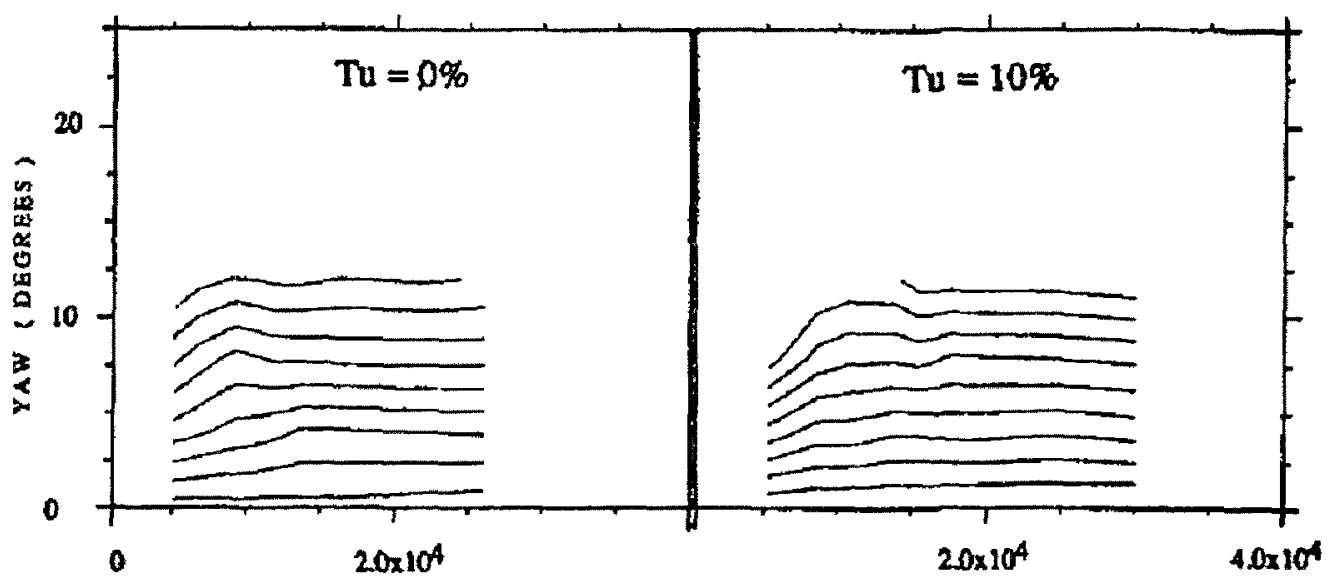

Figure 2.14: Turbulence Intensity Effects on the Yaw Coefficient of a Five-Hole Pressure Probe, from Dominy and Hodson (1993)

Takahashi (1997) provides a brief and mainly qualitative overview of the effect of turbulence and unsteadiness on measurements using seven-hole pressure probes. The author examined these effects by measuring the vortex shedding downstream of a cylinder in cross-flow. By comparing the seven-hole probe results to measurements taken using hot-wire anemometry, Takahashi found that reducing seven-hole probe data at high-turbulence using a low-turbulence calibration resulted in an overestimation of dynamic pressure when the flow's frequency content was above the limit of the pneumatic measuring system. Although the author is not clear, it seems implied that the measurements were taken with the probe aligned with the mean flow direction. He also found that flow angle measurements were unaffected when flow over the tip of the probe was attached but that the flow angle coefficients became very sensitive to small perturbations when the flow was not attached. Thus, measurements of large flow angles 
were subject to greater uncertainty. Results presented by Takahashi provide no mention of turbulence intensity effects on the measurement of total pressure.

Pisterman (2004) examined flow fields that consisted of large velocity gradients, turbulence intensities of up to about $10 \%$, and sometimes wall effects. Pisterman found that turbulence intensity and velocity gradients together increased the uncertainty of seven-hole probe measurements but did not examine either in isolation. Pisterman could not conclude if turbulence intensity alone had a significant effect on results due to the measurement uncertainty present in his results but did conclude that turbulence intensity effects are less than the effects of velocity gradients in the flows he investigated.

Available literature for turbulence effects on multi-hole probes does not discuss the influence of the integral length scale of turbulence.

\subsubsection{Influence of Wall Proximity}

Like with single-hole probes, multi-hole probes can distort or displace streamlines when near a body. This effect causes errors in near wall measurements. Tavoularis (2005) and Blake (1983) suggest that measurements be made at least two outer probe diameters from a wall.

In the present work, all data is collected well away from walls. Wall proximity effects are not considered in Chapter 4. 


\subsection{Turbulence Generation}

In research applications, the turbulence intensity and length scale of a flow are commonly controlled through the use of porous flow blockages such as grids, meshes, or honeycombs. As described by Roach (1987), these blockages have two effects on the flow: they alter the spectrum and thus the length scale of the turbulence, and their wakes contribute turbulent energy to the flow field. Depending on the dimensions of the blockage relative to the turbulence already present in the flow, these blockages can have the effect of increasing or decreasing the level of turbulence.

Laws and Livesey (1978) and Roach both summarize the use of grids to generate turbulence. Turbulence intensity and length scale downstream of a grid is determined by the properties of the upstream flow, the diameter and spacing of the bars in the grid, and downstream distance from the grid. Turbulence intensity increases with increasing grid diameter and decreasing downstream distance. Turbulent length scale increases for increasing grid diameter and increasing downstream distance. Both turbulence intensity and length scale are independent of the Reynolds number through the grid (Roach). According to Kurian and Fransson (2009) it is possible to control the resultant length scale independent of the turbulence intensity, but in practice this is difficult because they are still functions of the same grid dimensions.

Pinker and Herbert (1967) found pressure loss through grids to increase with increasing Reynolds number and decreasing porosity. According to Roach, pressure loss 
caused by grids generally limits the maximum operating velocity of an installation. To minimize pressure loss in their installation, Matsunuma et al. (1999) used perforated sheets to generate turbulence upstream of a flow contraction. Figure 2.15 is a schematic of the wind tunnel used by Matsunuma et al.; screens at positions 1,2, and 3 generated the turbulence. The flow contraction prior to the stator inlet allowed for a low velocity through the screens and a high velocity and turbulence intensity $(\sim 9 \%)$ at the test section. The flow contraction in this setup however will distort the turbulent eddies in the flow and result in anisotropic turbulence at the test section. Ertunc and Durst (2008) summarize the available literature on the distortion of turbulence through an axisymmetric convergence. This literature shows that a flow contraction causes an elongation of the turbulent eddies, a decrease in the streamwise components of turbulence, and an increase in the crosswise components.

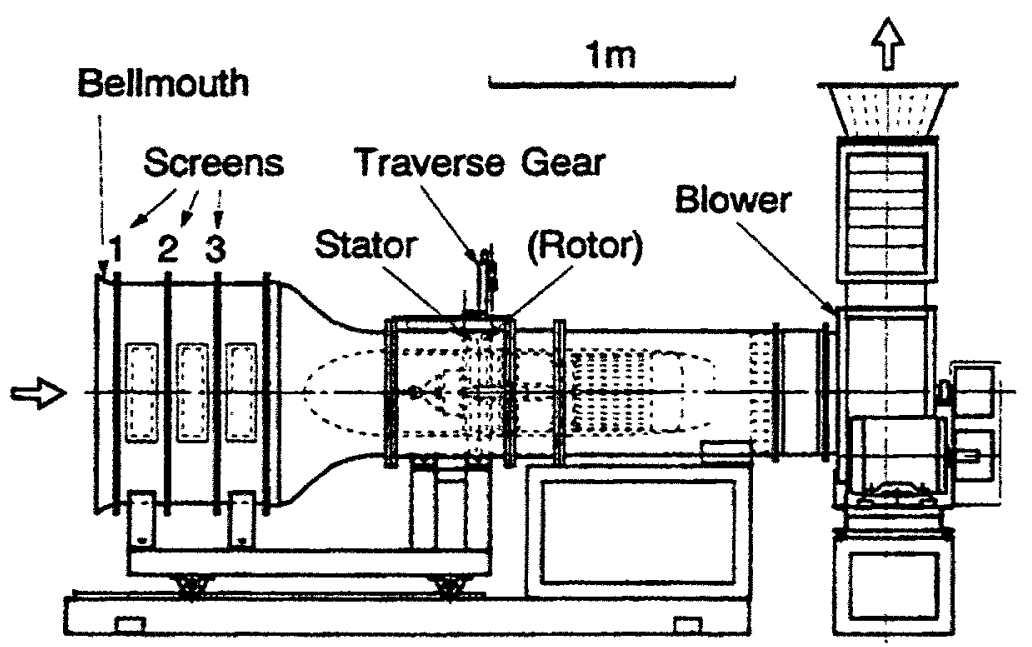

Figure 2.15: Turbulence Generation Upstream of a Flow Convergence, from Matsunuma et al. (1999) 
In addition to passive turbulence generation, active methods are reported by Gad-ElHak and Corrsin (1974) and Fransson et al. (2005). Both designed turbulence generating grids that could inject fluid into the main flow to increase turbulence production. The velocity of the injected flow could be varied to change the turbulence intensity. This method of turbulence generation was shown to be more effective than passive methods at producing turbulent flows with good isotropy. The drawback to this method however is a significant increase in complexity. 


\section{Chapter 3}

\section{Experimental Apparatus and Procedures}

\subsection{Introduction}

This Chapter describes the low-turbulence probe calibration rig and the instrumentation used in the current study. Probe calibration, data reduction, and data collection methods are also discussed. Modifications to the calibration rig to obtain high turbulence levels are described in Section 4.2.

\subsection{Low-Turbulence Probe Calibration Rig}

A schematic of the probe calibration rig used here is shown as Figure 3.1. This setup, originally designed by Benner (2003), was used for all calibrations and data collection at low turbulence. A downstream vacuum cleaner (not shown) provides the flow for the enclosed rig. The vacuum draws air through the inlet bellmouth and nozzle to the measurement plane one to two nozzle diameters downstream. Velocity at the probe location can be varied from 0 to $43 \mathrm{~m} / \mathrm{s}$. The streamwise turbulence intensity of the flow at this location is about $0.5 \%$. 


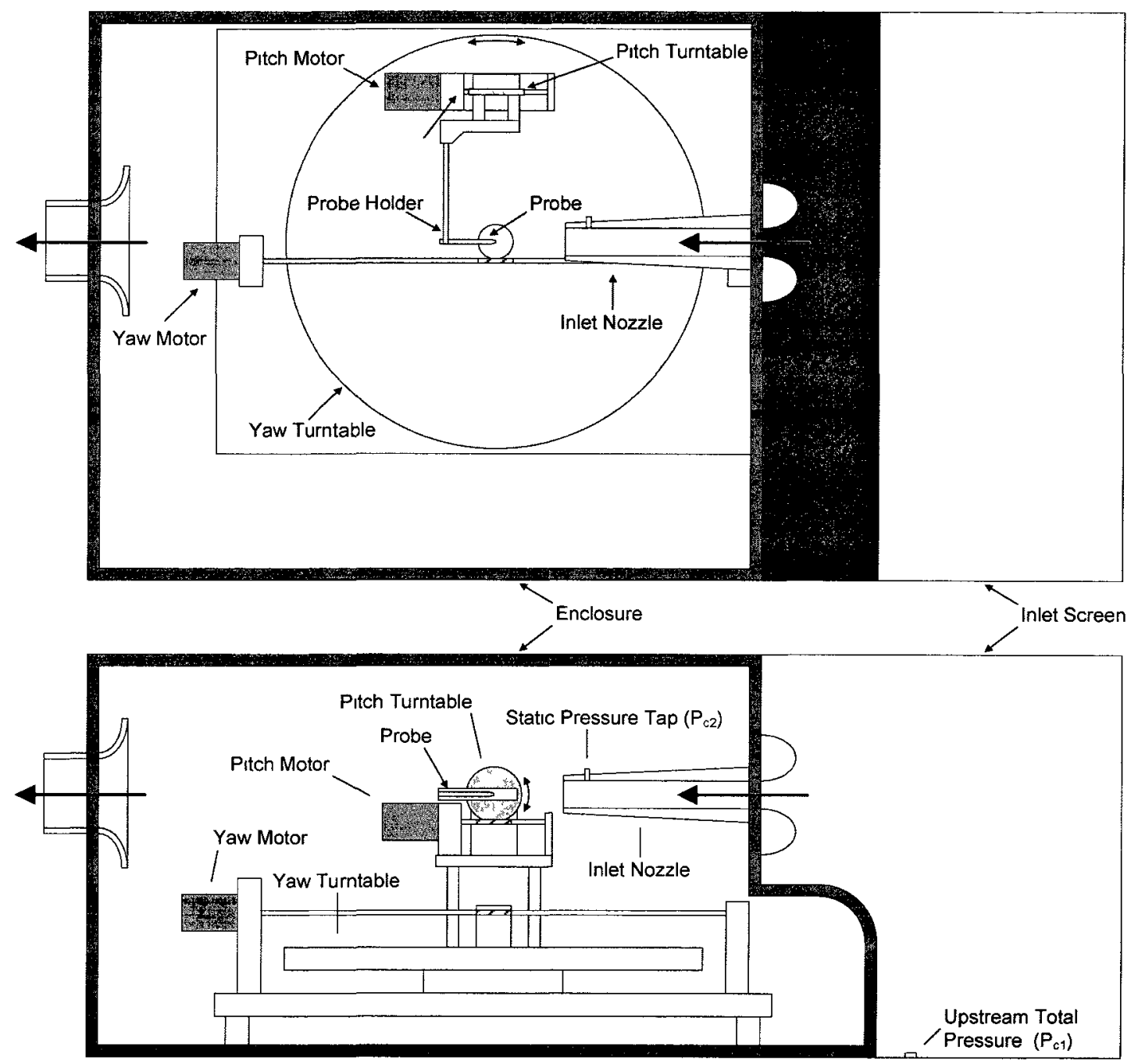

Figure 3.1: Schematic Diagram of the Low-Turbulence Probe Calibration Rig, adapted from Benner (2003)

The rig was calibrated at each operating point to relate the reference total and static pressures, $P_{c 1}$ and $P_{c 2}$, to the true total, static, and dynamic pressures at the probe. For this purpose, total and static pressures in the jet were measured at several Reynolds numbers to determine the values of: 


$$
\begin{gathered}
C_{P o C a l}=\frac{P_{o \text { True }}-P_{c 1}}{P_{c 1}-P_{c 2}} \\
C_{P s \text { Cal }}=\frac{P_{\text {cTrue }}-P_{c 2}}{P_{c 1}-P_{c 2}} \\
C_{q \text { Cal }}=\frac{P_{o \text { True }}-P_{s \text { True }}}{P_{c 1}-P_{c 2}}=\frac{q_{\text {True }}}{P_{c 1}-P_{c 2}}=1+C_{P o \text { Cal }}-C_{P s C a l}
\end{gathered}
$$

where $C_{P o C a l}, C_{P s C a l}$, and $C_{q C a l}$ are the calibration rig total pressure, static pressure, and dynamic pressure coefficients. All three coefficients were found to be relatively constant over all Reynolds numbers for low-turbulence operation. The values for these coefficients found here were $C_{P o C a l} \approx 1.5 \times 10^{-3}, C_{P s C a l} \approx 6.5 \times 10^{-3}$, and $C_{q C a l} \approx 0.992$. These values are in agreement with those obtained by Mahallati (2003) for the same apparatus.

The calibration rig produces an inviscid jet core at least $10 \mathrm{~mm}$ in diameter. Total pressure variation across the jet was less than $0.2 \%$ of dynamic pressure, as shown in Figure 3.2. Dynamic pressure and turbulence intensity had similar uniformity across the jet. These results agree with those of Benner and Mahallati for the same apparatus.

A two-axis motorized turntable built by Yaras (1990) controlled probe pitch and yaw. The probe was mounted on the pitch turntable which in turn was attached to the yaw turntable, as shown in Figure 3.1, providing two degrees of freedom. The probe tip is located at the centre of rotation of both turntables to prevent translation during pitch and yaw motion. Probe pitch and yaw angles are physically limited to approximately $\pm 60^{\circ}$ by 


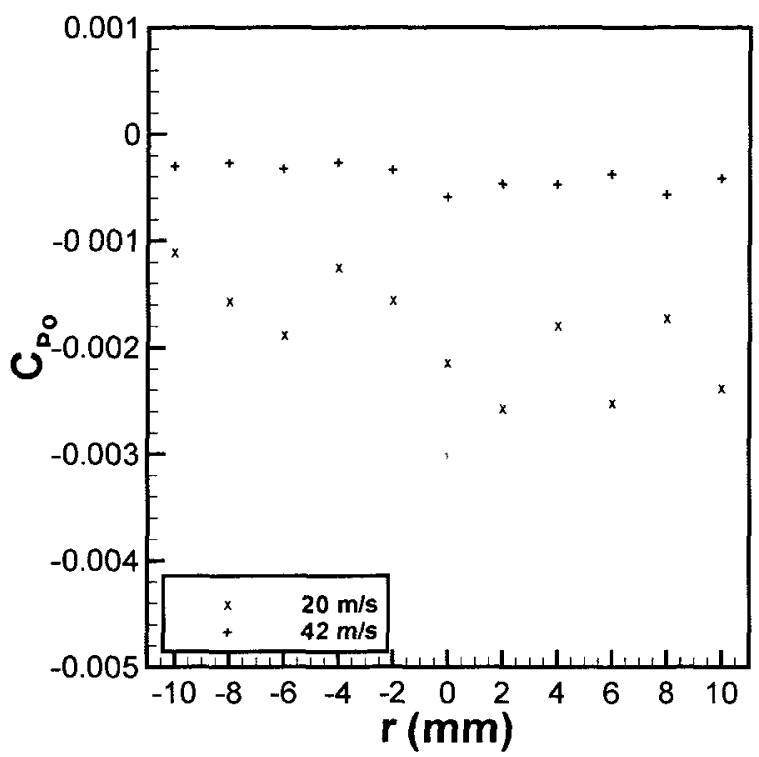

Figure 3.2: Profile of Total Pressure across the Calibration Jet at a Low Turbulence Intensity

the enclosure. Angular uncertainty was found to be less than $0.05^{\circ}$ by Yaras and Benner and minimal backlash was found in either turntable.

Figures 3.3 and 3.4 define the two probe coordinate systems used in the present study. Figure 3.3 shows the tangential coordinate system in terms of angles $\alpha$ and $\beta$, or as some references, such as Gerner et al. (1984), denote them, $\alpha_{T}$ and $\beta_{T} . \alpha$ is the angle between the component of velocity in the probe axis, $u$, and the projection of the resultant velocity, $V$, on the $u w$ plane. Similarly, $\beta$ is the angle between $u$ and the projection of $V$ on the $u v$ plane. Described another way, $\alpha$ and $\beta$ are the angles of rotation for the resultant velocity vector about the $y$ - and z-axes, respectively, in the probe coordinate system. Figure 3.4 shows the polar coordinate system in roll angle $\phi$ and misalignment $\theta$. 


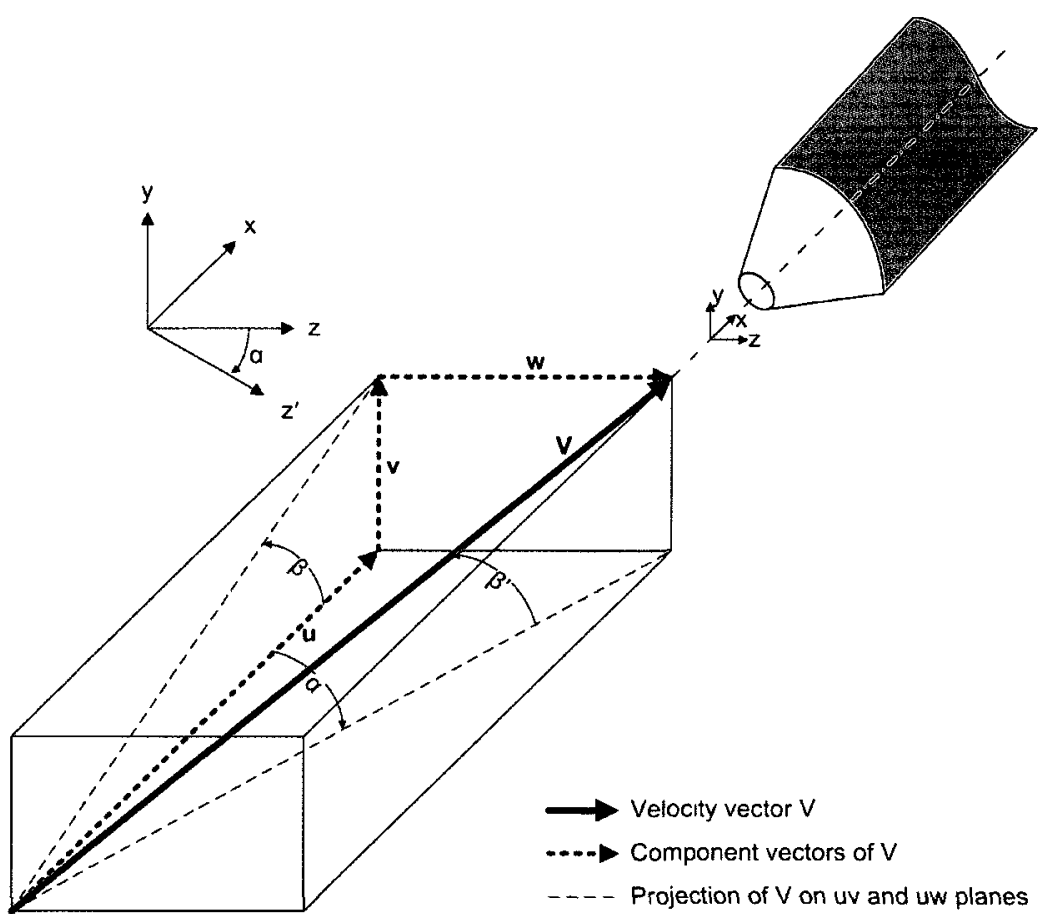

Figure 3.3: Probe Tangential Coordinate System, adapted from Gerner et al. (1984)

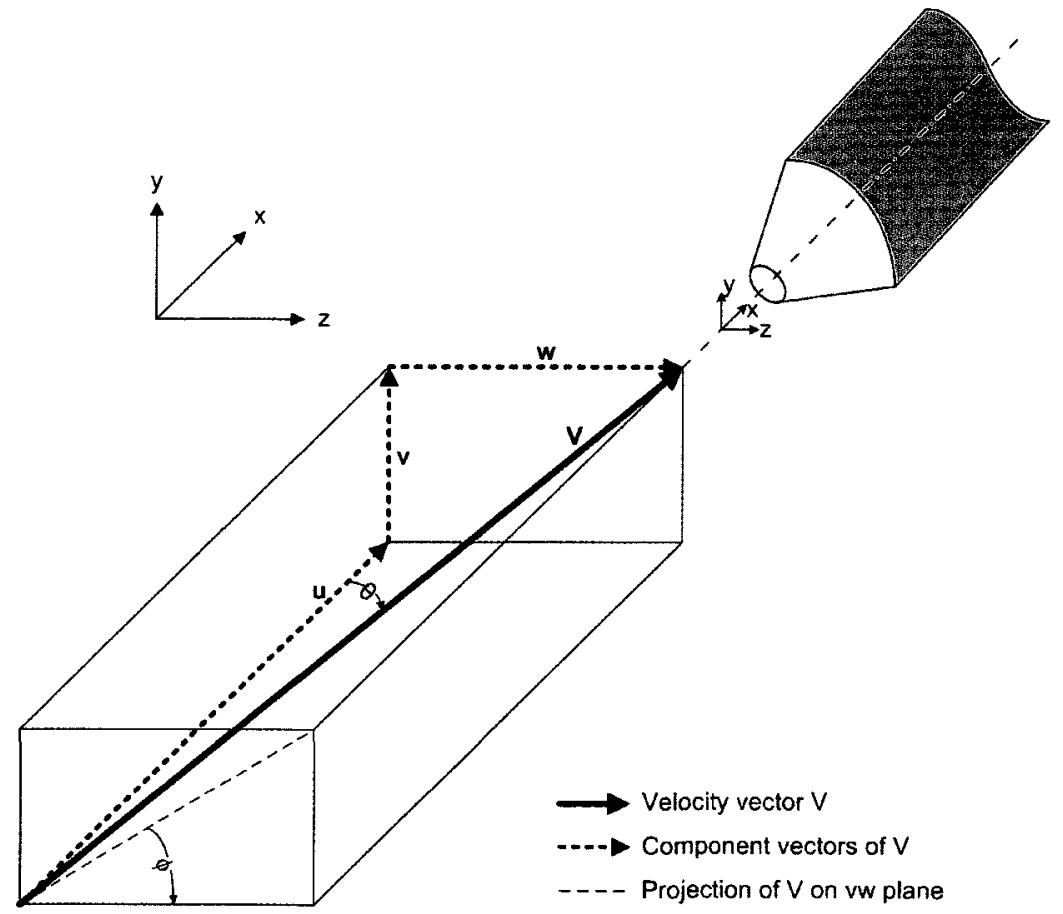

Figure 3.4: Probe Polar Coordinate System, adapted from Gerner et al. (1984) 
It should be noted that, for the two-axis system described here, probe pitch control is coupled to yaw control. The yaw turntable is fixed to ground and always rotates about the y-axis in Figure 3.3. Thus, the yaw turntable independently varies $\alpha$. The pitch turntable however is fixed to the yaw turntable and therefore rotates about the $\mathrm{z}^{\prime}$-axis. This rotation is indicated on the figure by $\beta^{\prime}$. Hence, the tangential coordinate $\beta$ is a function of both pitch and yaw turntable angles according to:

$$
\begin{gathered}
\beta=\tan ^{-1}\left(\frac{\tan \beta^{\prime}}{\cos \alpha}\right) \\
\beta^{\prime}=\tan ^{-1}(\tan \beta \cdot \cos \alpha)
\end{gathered}
$$

\subsection{Instrumentation}

\subsubsection{Data Acquisition System}

Data acquisition used a Windows based computer equipped with a United Electronic Industries PowerDAQ PD2-MFS-8-800/14 data acquisition card. This simultaneous sample-and-hold card can sample 8 analog voltage inputs at a rate of $800 \mathrm{kHz}$ with 14-bit resolution over $\pm 10 \mathrm{~V}$. Two 12-bit analog outputs are also available. The present author and several present and past researchers developed a user interface in Labview to manage all data acquisition and motor controller functions. Specific components of the data acquisition system, such as the temperature monitor, digital barometer, pressure transducers, and probes are described in more detail below. 


\subsubsection{Temperature Monitor}

An Omega DPi8-C24 temperature monitor connected to a type-T (CopperConstantan) thermocouple measured temperature inside the calibration rig during testing. This monitor has a stated accuracy of $\pm 0.4^{\circ} \mathrm{C}$. Data were transferred to the data acquisition system through an RS-232 port. Temperature measurements were used to determine air viscosity during testing.

\subsubsection{Digital Barometer}

An Omega DPI740 digital barometer measured ambient pressure during testing. This barometer has an accuracy of $\pm 0.02 \%$ of the full scale over a range of 77 to $115 \mathrm{kPa}$ (absolute). Data were transferred to the data acquisition system through an RS-232 port. Ambient pressure readings were used along with temperature measurements to determine flow density during testing.

\subsubsection{Hot-wire Anemometer and Probes}

The hot-wire anemometer system and probes used here are described below. This description draws on discussion by Mahallati (2003). Also included is discussion of the basic principles of hot-wire anemometry based on the work of Bruun (1995).

Hot-wire anemometry infers flow velocity across a heated wire from the flow-induced cooling of the wire. The A.A. Lab Systems AN-1003 anemometer used here maintains hot-wire sensors at constant temperature using a Wheatstone Bridge. A 
schematic drawing of this arrangement is shown in Figure 3.5 which is reproduced from Mahallati. The top of bridge voltage $\left(E_{T O B}\right)$ is proportional to the velocity across the wire. Anemometer output $\left(E_{\text {out }}\right)$ is related to the top of bridge voltage through:

$$
E_{\text {out }}=G\left(E_{T O B}-E_{\text {off }}\right)
$$

Output can be adjusted through a user defined gain, $G$, and voltage offset, $E_{\text {off }}$. Typical gain and offset values for the current study were 3.3 and $-5.5 \mathrm{~V}$, respectively, to maintain system output within $\pm 10 \mathrm{~V}$.

The present study used several single-normal hot-wire probes and a single X-wire hot-wire probe. Figure 3.6 shows the dimensions of the probes used here; all single-normal probes had approximately the same dimensions. The frequency response of these hot-wire probes is a function of the wire overheat ratio, $O H R=R_{w} / R_{r}$, and latencies in the anemometry system. Higher overheat ratios lead to higher frequency response but can cause wire oxidization or breakage. Typical values of $O H R$ in air range from 1.3 and 2. Using guidance from Bruun, the current study sampled hot-wire data at $28 \mathrm{kHz}$ with a low-pass filter of $14 \mathrm{kHz}$ and $O H R=1.5$.

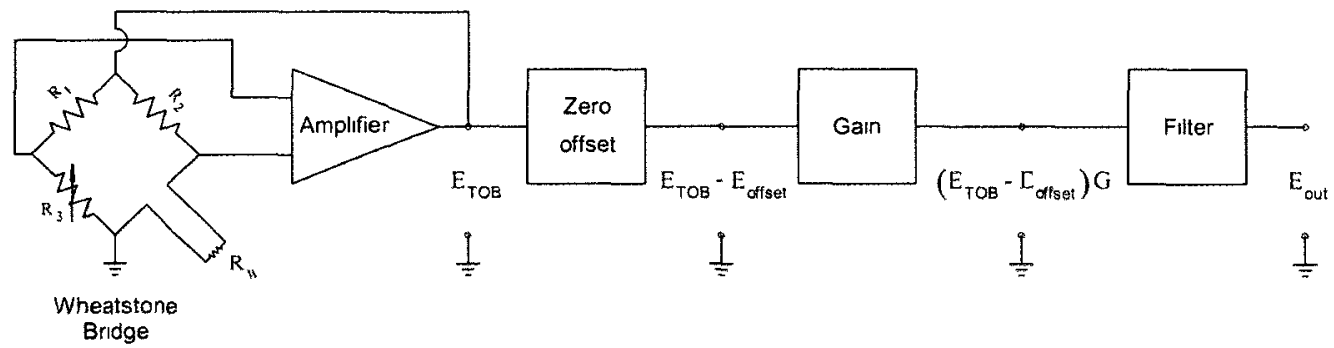

Figure 3.5: Constant Temperature Anemometer Block Diagram, from Mahallati (2003) 
a) Single-normal

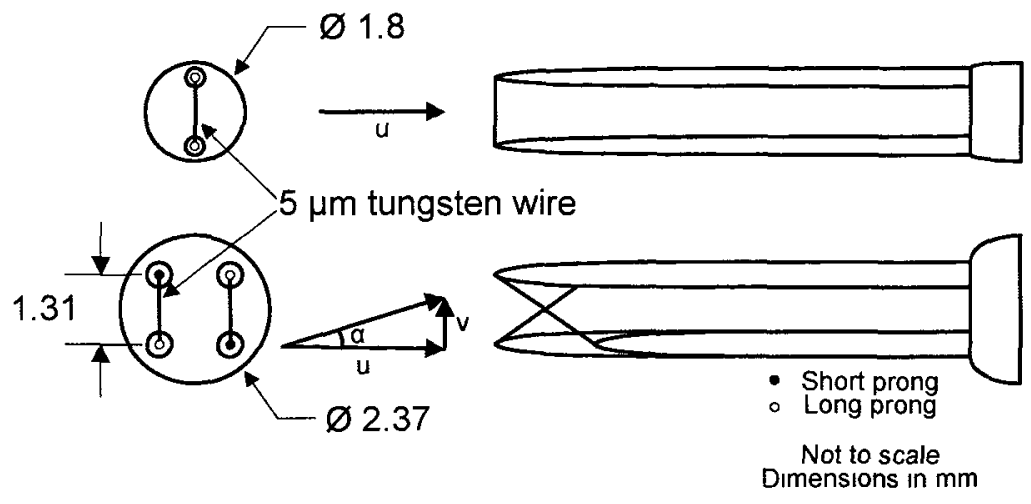

Figure 3.6: Hot-wire Probes

\subsubsection{Pressure Transducers}

The current study used eight Data Instruments DRAL505DN differential pressure transducers. These transducers have a range of $\pm 1250 \mathrm{~Pa}$ with quoted hysteresis error of less than $\pm 0.25 \%$ full scale. The transducers are temperature compensated over a range of 0 to $50^{\circ} \mathrm{C}$. A common $14 \mathrm{VDC}$ power supply powered all transducers; all transducers were attached to the same electrical ground. Output from the transducers was 1 to 6VDC. All transducers were calibrated prior to data collection using the micromanometer designed by Benner (2003) and Mahallati (2003). Calibration procedures and typical calibration coefficients for the transducers are included in Appendix A. Transducer calibrations were checked regularly during testing. 


\subsubsection{Pressure Probes}

\subsubsection{Kiel Probe}

A Kiel probe consists of a Pitot tube surrounded by an outer sheath. The sheath aligns the incoming flow with the Pitot tube to allow for total pressure measurement in misaligned flows. As discussed in Section 2.2.3, Kiel probes typically measure true total pressure at up to $35^{\circ}$ of misalignment. Standard Kiel probes vent flow straight through the sheath along the probe axis. However, the probe used in the current study vents using two holes in the sides of the sheath, as shown in Figure 3.7.

\subsubsection{Three-Hole Pressure Probe}

The three-hole pressure probe designed by Islam (1999) was used here. The probe consists of three hypodermic stainless steel tubes soldered together as shown in Figure 3.8. The outer hypodermic tubes were chamfered; solder was added prior to chamfering to allow for a smooth probe tip. Each tube was connected to its own pressure transducer to measure all pressures simultaneously.

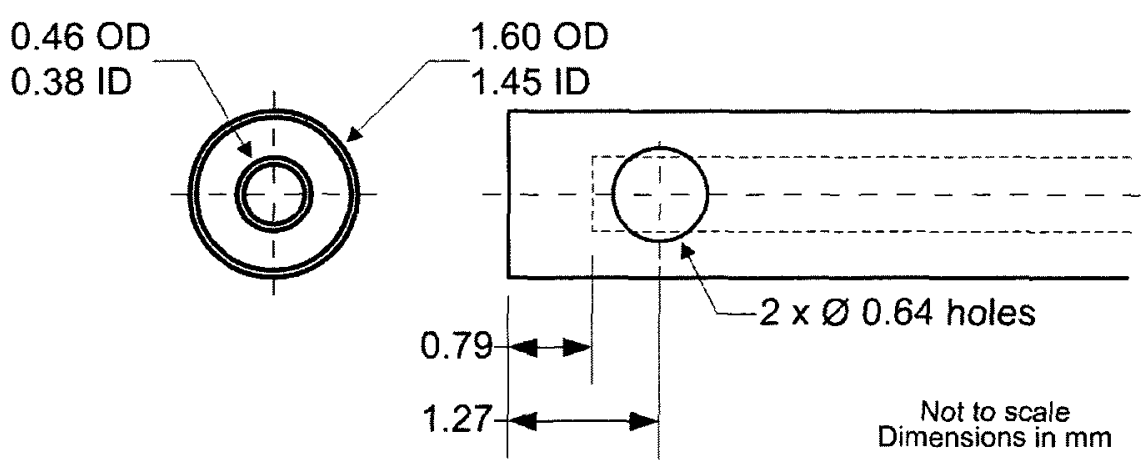

Figure 3.7: Modified Kiel Probe, adapted from Taremi (2005) 


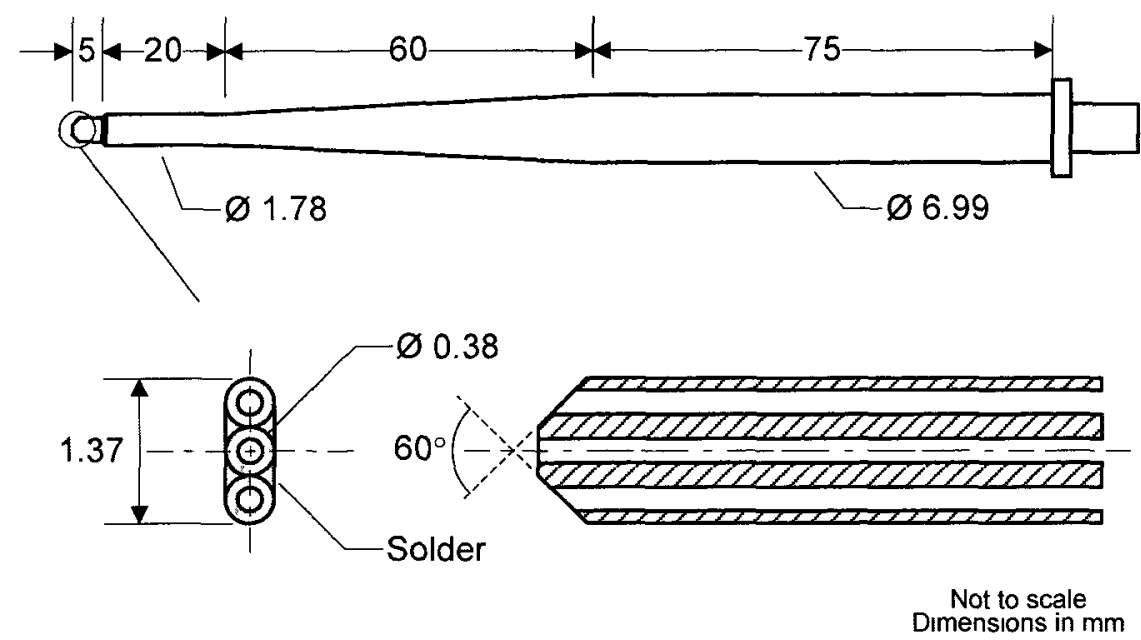

Figure 3.8: Three-hole Pressure Probe

\subsubsection{Seven-Hole Pressure Probes}

Two seven-hole pressure probes, 7HP1 and 7HP2, were used here. Figures 3.9 and 3.10 show the probes, respectively. Each probe is a bundle of seven hypodermic stainless steel tubes inside a stainless steel housing. Six tubes were soldered in $60^{\circ}$ increments circumferentially around the central tube in a natural bundle. The outer tubes were chamfered to a cone angle of $60^{\circ}$; gaps between tubes at the probe tip were filled with solder before chamfering to create a smooth cone. Each tube was connected to its own pressure transducer to measure all pressures simultaneously. The tip of probe 7HP1 is smaller than that of 7HP2. 7HP1 also has a gooseneck to aid in near-wall measurements for other research. 


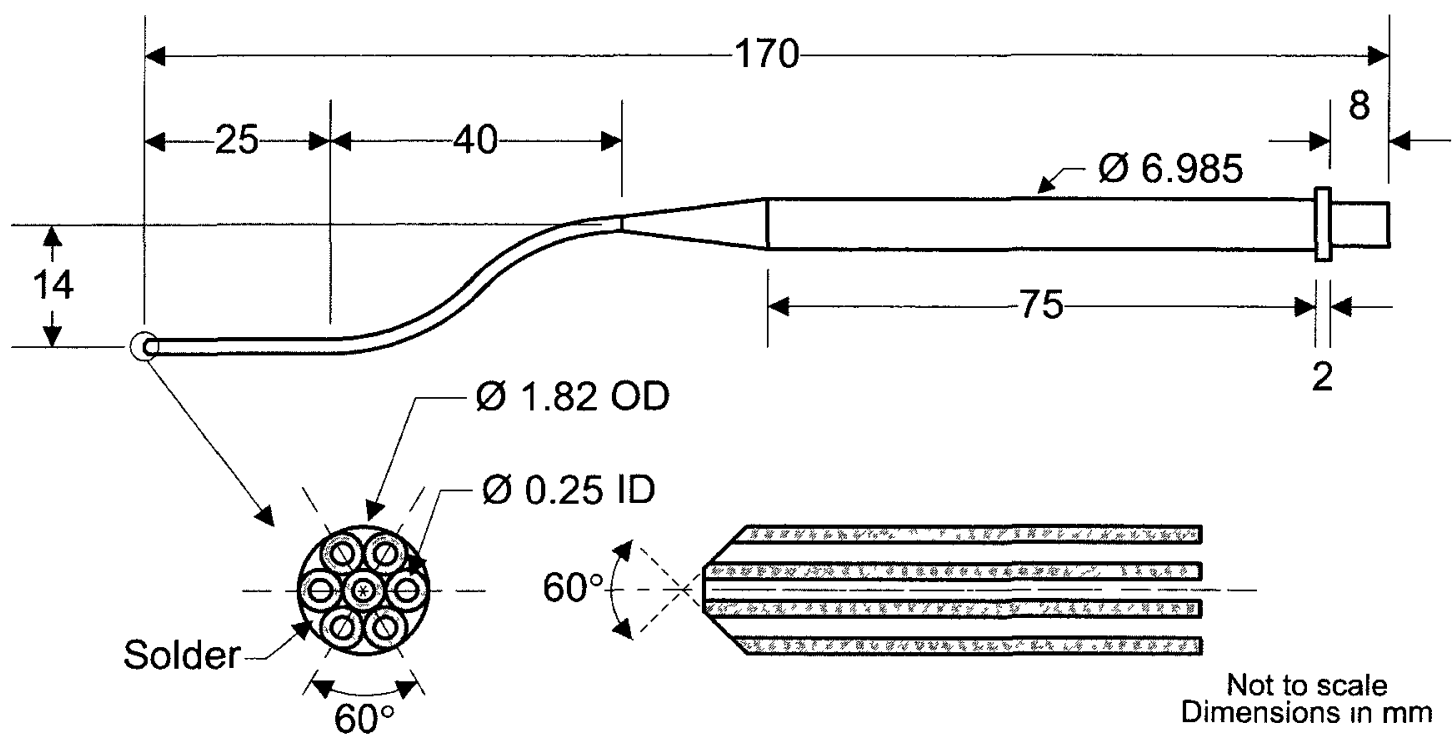

Figure 3.9: Seven-Hole Pressure Probe 7HP1

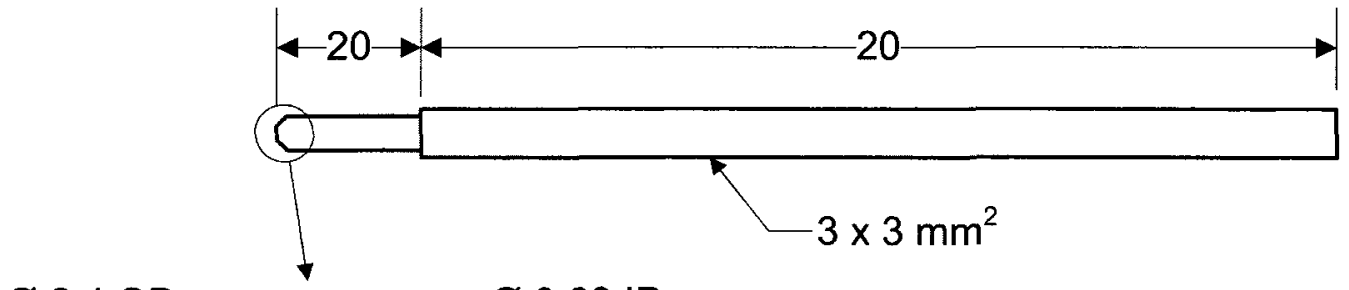

$\varnothing 2.4 \mathrm{OD}$

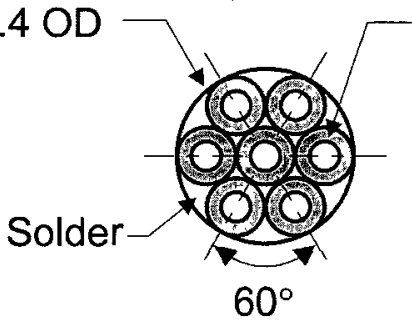

$\varnothing 0.33$ ID

Figure 3.10: Seven-Hole Pressure Probe 7HP2

Not to scale Dimensions in $\mathrm{mm}$

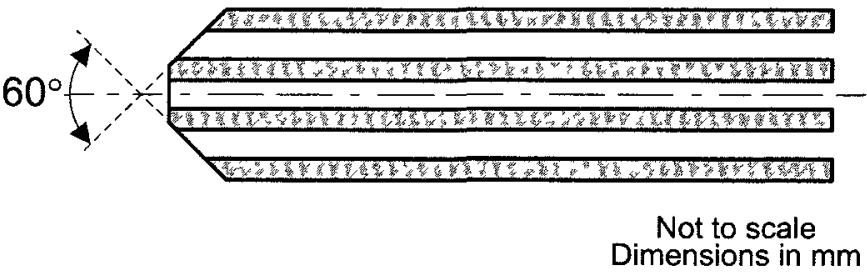




\subsection{Probe Calibration, Data Reduction, and Measurement Uncertainty}

\subsubsection{Single-Normal Hot-wire Probe}

The single-normal hot-wire probes used here were calibrated immediately before any flow measurements using the methods of Bruun (1995). Calibrations consisted of 20 to 30 data points taken over a velocity range of 4 to $43 \mathrm{~m} / \mathrm{s}$. Probes were always aligned with the mean flow direction. Anemometer response was curve fit using King's Law:

$$
E_{T O B}^{2}=A+B V_{e f f}^{n}
$$

where the effective cooling velocity, $V_{\text {eff, }}$, is essentially streamwise velocity and $A, B$, and $n$ are calibration coefficients. Ambient temperature was monitored during testing to ensure there was minimal temperature variation. Figure 3.11 shows three typical calibrations. Bruun estimated that the uncertainty in the results for velocity due to the curve fitting procedure is less than $\pm 1 \%$ for flows with turbulence intensities less than about $15 \%$. The present author estimates repeatability error here to be about $\pm 1 \%$ based on repeated use of the apparatus. Using the root-sum-of-squares method of Taylor and Kuyatt (1994), single-normal hot-wire velocity measurement uncertainty is thus estimated to be less than $\pm 1.5 \%$. 


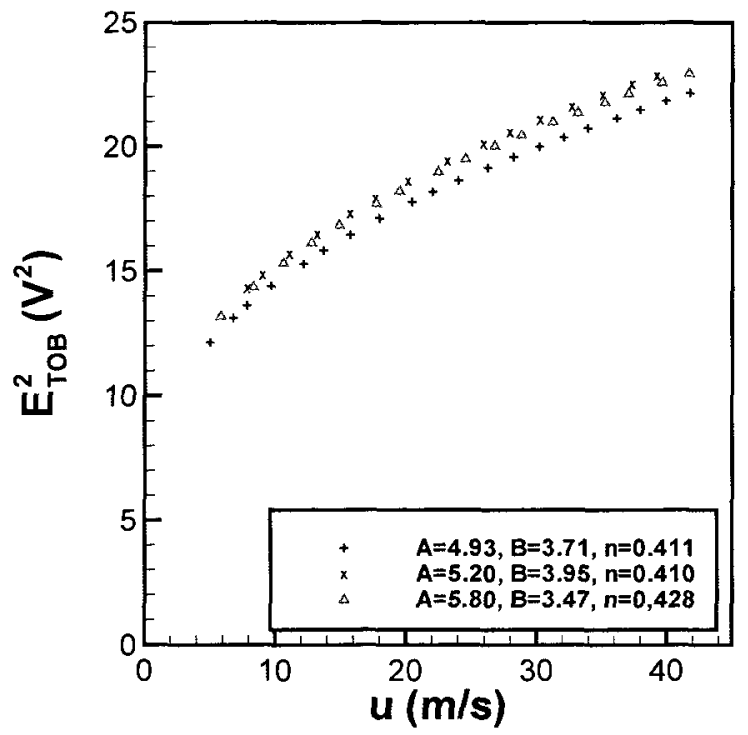

Figure 3.11: Sample Calibration Curves for Single-Normal Hot-wire Probes

Streamwise turbulence intensity, $T u_{u}$, can be obtained from single-normal hot-wire data as follows:

$$
T u_{u}=\frac{\sqrt{\overline{u^{\prime 2}}}}{\bar{V}}
$$

Where $u^{\prime}$ represents the instantaneous velocity fluctuations in the streamwise direction and $\bar{V}$ is the mean flow velocity. The integral length scale of turbulence in the streamwise direction, $\Lambda_{x}$, can be obtained through the methods of Hinze (1975). Using the Taylor frozen-turbulence-pattern hypothesis the integral length scale, also known as the typical large eddy size, is obtained from the autocorrelation of the fluctuating velocity signal. The autocorrelation function is: 


$$
R(\theta)=\frac{\overline{u^{\prime}(t) u^{\prime}(t+\theta)}}{\overline{u^{\prime 2}}}=\frac{\frac{1}{\Delta t} \int_{0}^{\Delta t} u^{\prime}(t) u^{\prime}(t+\theta) d t}{\frac{1}{\Delta t} \int_{0}^{\Delta t} u^{\prime 2}(t) d t}
$$

where $\theta$ is the offset time. Figure 3.12 shows a sample profile of the autocorrelation function. From this relation, the integral length scale can be approximated by:

$$
\Lambda=\bar{U} \int_{0}^{\infty} R(\theta) d \theta
$$

where the integral is typically evaluated until $R(\theta)$ is less than zero. As can be seen in the figure, scatter can play a significant role in determining the point at which $R(\theta)=0$. For data where $R(\theta)$ approached but did not immediately cross zero, autocorrelation results were inspected manually to estimate the likely crossing point in the absence of scatter.

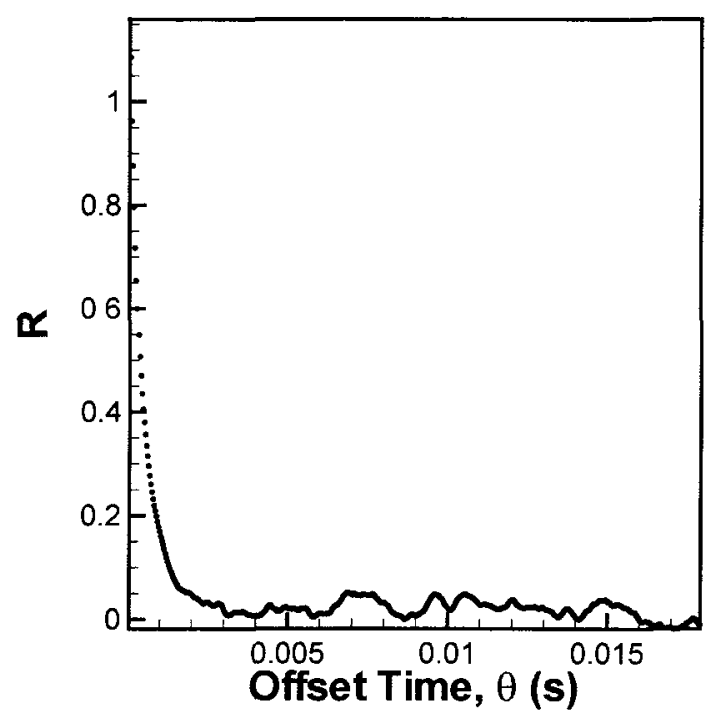

Figure 3.12: Sample Autocorrelation Function 


\subsubsection{X-Wire Type Hot-wire Probe}

The methods and data reduction software of MacIsaac et al. (2009) and MacIsaac (2010) were used here for $\mathrm{X}$-wire probe calibration and data reduction. MacIsaac modified the quadruple-wire probe calibration method of Döbbeling et al. (1990) to calibrate a X-wire probe in multiple roll orientations. Calibration consisted of two parts: a velocity calibration like that discussed in Section 3.4.1 for single-normal hot-wire probes, and a directional calibration.

The directional calibration was performed over $\pm 45^{\circ}$ in pitch and yaw in $2^{\circ}$ increments to find a directional sensitivity function for each wire. The effective cooling velocity in Equation 3.7 for each wire is related to the resultant flow velocity through the directional sensitivity functions. By collecting data in $0^{\circ}, 45^{\circ}$, and $90^{\circ}$ roll orientations MacIsaac simulated a time-averaged six-wire probe and used this to determine time averaged three-dimensional velocities and flow angles. The reader should note that the measurement of all three orientations is not simultaneous. Thus, this method can only determine the time-averaged velocities from a series of samples and not the instantaneous velocity for each sample. MacIsaac also used the procedures of Buresti and Di Cocco (1987) to obtain the Reynolds stresses and thus the flow turbulence from the X-wire data.

Table 3.1 summarizes the measurement uncertainties estimated by Buresti and Di Cocco. These include error from the data reduction technique and their positioning 
errors. Positioning errors in the current study are less than those obtained by Buresti and Di Cocco, but their overall uncertainties serve as conservative estimates.

\begin{tabular}{|c|c|c|}
\cline { 2 - 3 } \multicolumn{1}{c|}{} & $\mathrm{Tu}=10 \%$ & $\mathrm{Tu}=20 \%$ \\
\hline Mean Velocity (\%) & 1.2 & 1.7 \\
\hline Angle $\left({ }^{\circ}\right)$ & 0.77 & 0.87 \\
\hline
\end{tabular}

Table 3.1: X-Wire Hot-wire Probe Measurement Uncertainty, from Buresti and Di Cocco (1987)

\subsubsection{Three-Hole Pressure Probe}

The three-hole probe calibration and data reduction procedures of Benner (2003) were applied here. These procedures were based on those of Lewis (1966). Calibrations consisted of 61 points over the range of $\pm 30^{\circ}$ in yaw in $1^{\circ}$ increments. The probe was aligned in pitch for all data. Figure 3.13 shows the numbering convention for the threehole probe. Flow angle is considered positive for $P_{3}>P_{2}$. Pressure measurements from the three ports during calibration were used to find the following calibration coefficients:

$$
\begin{gathered}
K_{\phi 2}=\frac{P_{1}-P_{2}}{P_{1}-P_{3}} \\
K_{\phi 3}=\frac{P_{1}-P_{3}}{P_{1}-P_{2}} \\
K_{12}=\frac{P_{1}-P_{2}}{q}
\end{gathered}
$$




$$
\begin{gathered}
K_{13}=\frac{P_{1}-P_{3}}{q} \\
K_{1}=\frac{P_{1}-P}{q}
\end{gathered}
$$

where $P_{1}$ through $P_{3}$ are the measured port pressures and $P$ and $q$ are the local static and dynamic pressures. Figures 3.14 through 3.16 show examples of typical three-hole probe calibration curves. Although $K_{\phi 2}$ and $K_{\phi 3}$ are both defined over the full yaw range, Benner shows that $K_{\phi 2}$ is more suitable for obtaining the flow angle at negative flow angles and $K_{\phi 3}$ at positive angles due to their sensitivities to measurement uncertainty. Similarly, Figure 3.15 demonstrates that $K_{12}$ and $K_{13}$ are unsuitable for flow angles greater than about $15^{\circ}$ and less than about $-15^{\circ}$, respectively, since the coefficients are double valued in those ranges.

For data reduction, $K_{\phi 2}$ and $K_{\phi 3}$ are calculated using the measured port pressures at each data point. The more appropriate coefficient is chosen based on their relative magnitudes: if $K_{\phi 2}>K_{\phi 3}, K_{\phi 3}$ is used to find flow angle; if $K_{\phi 2}<K_{\phi 3}, K_{\phi 2}$ is used. The value of $K_{\phi 2}$ (or $K_{\phi 3}$ ) is then compared to the calibration curve in Figure 3.14 to infer the flow angle for the data point. Using this flow angle, the values of $K_{12}$ (or $K_{13}$ ) and $K_{1}$ are then interpolated for using the calibration curves Figures 3.15 and 3.16. Finally, dynamic and static pressures are calculated from these coefficients. Total pressure is found through the summation of dynamic and static pressure. 

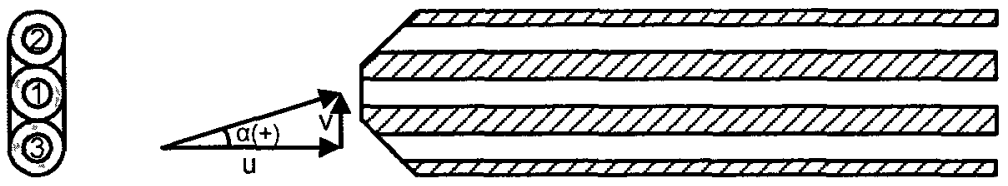

Figure 3.13: Three-Hole Pressure Probe Port Numbering Convention

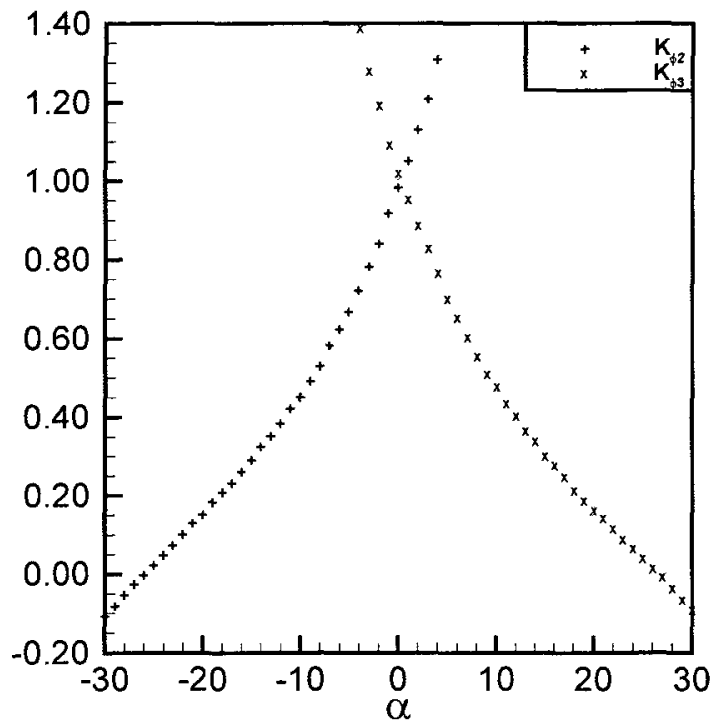

Figure 3.14: Sample $K_{\phi 2}$ and $K_{\phi 3}$ Calibration Curves for the Three-Hole Pressure Probe

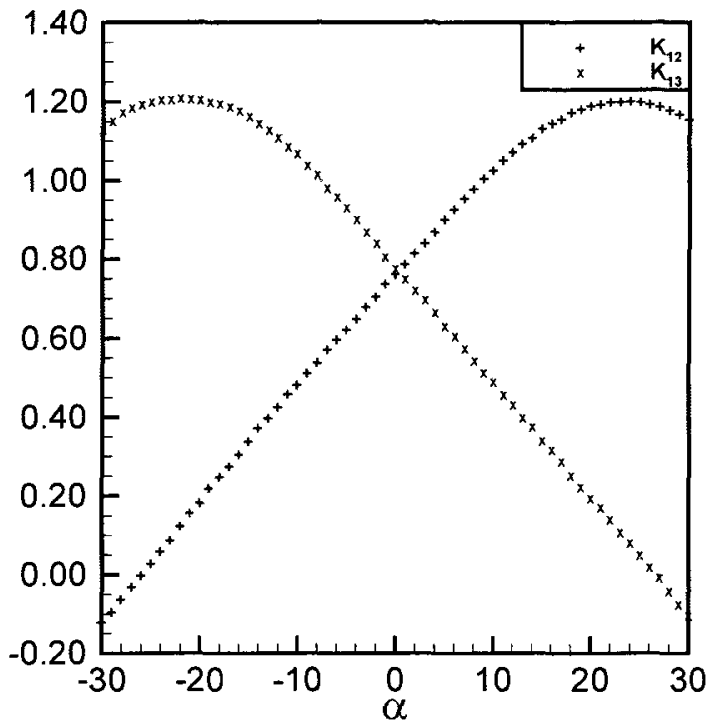

Figure 3.15: Sample $K_{12}$ and $K_{13}$ Calibration Curves for the Three-Hole Pressure Probe 


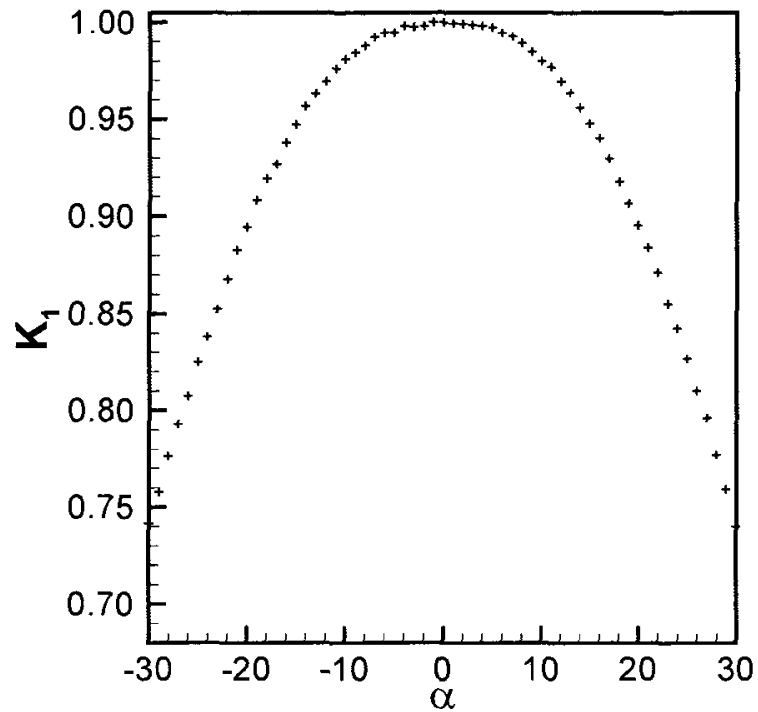

Figure 3.16: Sample $K_{1}$ Calibration Curve for the Three-hole Pressure Probe

The experimental uncertainty in the three-hole probe measurements was determined using the root-sum-of-squares method from Taylor and Kuyatt (1994) to combine the effects of all sources of error. These sources included the precision error due to fluctuations in the flow and the bias errors in the pressure transducers. To identify the precision error, data were collected in a known flow with a calibrated three-hole probe. The differences between the results inferred from these measurements and the true flow properties indicated the precision error at that operating point. This procedure was repeated multiple times at each Reynolds number and turbulence intensity investigated in the present study; the combined results of these tests were used to assess the overall precision errors. 
Table 3.2 presents the uncertainty in the three-hole probe measurements. As can be seen from the table, uncertainty in the measurements increased with turbulence intensity. This was due to an increase in the precision error as turbulence intensity increased; all other sources of error were relatively constant for all operating points. Reynolds number did not affect precision error in the range investigated here $\left(1850<R e_{D}<4100\right)$.

\begin{tabular}{|c|c|c|c|c|c|}
\cline { 2 - 6 } \multicolumn{1}{c|}{} & \multicolumn{5}{c|}{ Uncertainty (all Reynolds numbers) } \\
\cline { 2 - 6 } \multicolumn{1}{c|}{} & $T u=1.5 \%$ & $T u=3 \%$ & $T u=5 \%$ & $T u=8 \%$ & $T u=12 \%$ \\
\hline $\begin{array}{c}\text { Total Pressure } \\
(\% \text { of true dynamic pressure) }\end{array}$ & \pm 0.3 & \pm 0.3 & \pm 0.6 & \pm 0.6 & \pm 1.0 \\
\hline $\begin{array}{c}\text { Dynamic Pressure } \\
(\% \text { of true dynamic pressure) }\end{array}$ & \pm 0.5 & \pm 0.5 & \pm 0.7 & \pm 0.7 & \pm 1.1 \\
\hline Flow Angle $\left({ }^{\circ}\right)$ & \pm 0.5 & \pm 0.5 & \pm 0.7 & \pm 0.7 & \pm 1.1 \\
\hline
\end{tabular}

Table 3.2: Three-Hole Pressure Probe Measurement Uncertainty

To minimize the effect of the increase in precision error, the sample times used for data collection at the higher turbulence intensities were also increased. By collecting longer samples for each data point, the effects of the random fluctuations in the flow can be partially damped out. Longer samples are more representative of the true timeaveraged value. The uncertainties shown in Table 3.2 were established using the sample times listed in Table 3.3. Greater increases in the sample times could have further reduced the precision errors, but this was deemed impractical given the time required to collect each data set. 


\begin{tabular}{|c|c|}
\hline$T u(\%)$ & Sample Length $(\mathrm{s})$ \\
\hline 1.5 & 10 \\
\hline 3 & 30 \\
\hline 5 & 40 \\
\hline 8 & 55 \\
\hline 12 & 60 \\
\hline
\end{tabular}

Table 3.3: Pressure Probe Data Sample Lengths

\subsubsection{Seven-Hole Pressure Probe}

Seven-hole pressure probe calibration procedures originate from Gallington (1980). Everett et al. (1983), Gerner et al. (1984), Yaras (1990), Zilliac (1993), DeCecco (1995), and Benner (2003) applied methods similar to Gallington's with different interpolation schemes. Calibration procedures exist for both low-angle $\left(\alpha, \beta<20-30^{\circ}\right)$ and high-angle $\left(\alpha\right.$ or $\left.\beta>20-30^{\circ}\right)$ regimes. The procedures outlined here are taken from Benner and focus only on the low-angle regime. Probe response in the high-angle regime was omitted from the present study to simplify the analysis and shorten the time required for testing.

Figure 3.17 shows the port number convention used for the seven-hole probe. Positive yaw angles result in $P_{4}>P_{1}$. Positive pitch angles result in $P_{2}, P_{3}>P_{5}, P_{6}$. Seven-hole probe calibrations each consisted of a 961 -point grid over $\pm 30^{\circ}$ in pitch and yaw in $2^{\circ}$ increments. Calibration data were used to find:

$$
C_{\alpha 1}=\frac{P_{4}-P_{1}}{P_{7}-\overline{P_{1-6}}}
$$




$$
\begin{gathered}
C_{\alpha 2}=\frac{P_{3}-P_{6}}{P_{7}-\overline{P_{1-6}}} \\
C_{\alpha 3}=\frac{P_{2}-P_{5}}{P_{7}-\overline{P_{1-6}}}
\end{gathered}
$$

where $P_{1}$ through $P_{7}$ are the port pressures and:

$$
\overline{P_{1-6}}=\frac{\left(P_{1}+P_{2}+P_{3}+P_{4}+P_{5}+P_{6}\right)}{6}
$$

In the low-angle regime, $P_{7}-\overline{P_{1-6}}$ is referred to as the "pseudo-dynamic" pressure since $\overline{P_{1-6}}$ approximates the local static pressure and $P_{7}$ approximates the total pressure. The numerators in Equations 3.16 to 3.18 use opposing port pressures. Thus, each coefficient is most sensitive to flow angle along the plane of those ports.

Using Equations 3.16 through 3.18 we define:

$$
\begin{gathered}
C_{\alpha}=\frac{2 C_{\alpha 1}+C_{\alpha 2}-C_{\alpha 3}}{3} \\
C_{\beta}=\frac{C_{\alpha 2}+C_{\alpha 3}}{\sqrt{3}}
\end{gathered}
$$
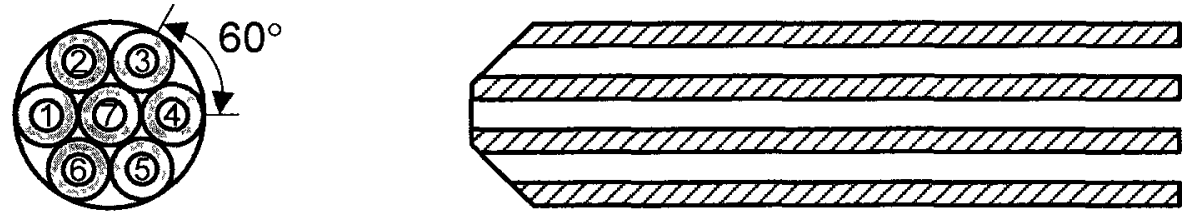

Figure 3.17: Seven-Hole Pressure Probe Port Numbering Convention 
as the coefficients of yaw and pitch, respectively. We can then find:

$$
\begin{gathered}
\alpha=f_{1}\left(C_{\alpha}, C_{\beta}\right) \\
\beta=f_{2}\left(C_{\alpha}, C_{\beta}\right) \\
C_{q 7 h p}=f_{3}\left(C_{\alpha}, C_{\beta}\right) \\
C_{P_{o} 7 h p}=f_{4}\left(C_{\alpha}, C_{\beta}\right)
\end{gathered}
$$

where the dynamic and total pressure coefficients of the seven-hole probe are defined as:

$$
\begin{gathered}
C_{q 7 h p}=\frac{P_{7}-\overline{P_{1-6}}}{P_{o}-P} \\
C_{P_{0} 7 h p}=\frac{P_{7}-P_{o}}{P_{7}-\overline{P_{1-6}}}
\end{gathered}
$$

The values of the coefficients $C_{\alpha}, C_{\beta}, C_{P o 7 h p}$, and $C_{q 7 h p}$ calculated using sample calibration data are included in Figures 3.18 through 3.21. Subplots show the value of each coefficient along $\alpha=0$ and $\beta=0$ with three separate calibrations overlaid to demonstrate the repeatability error. 


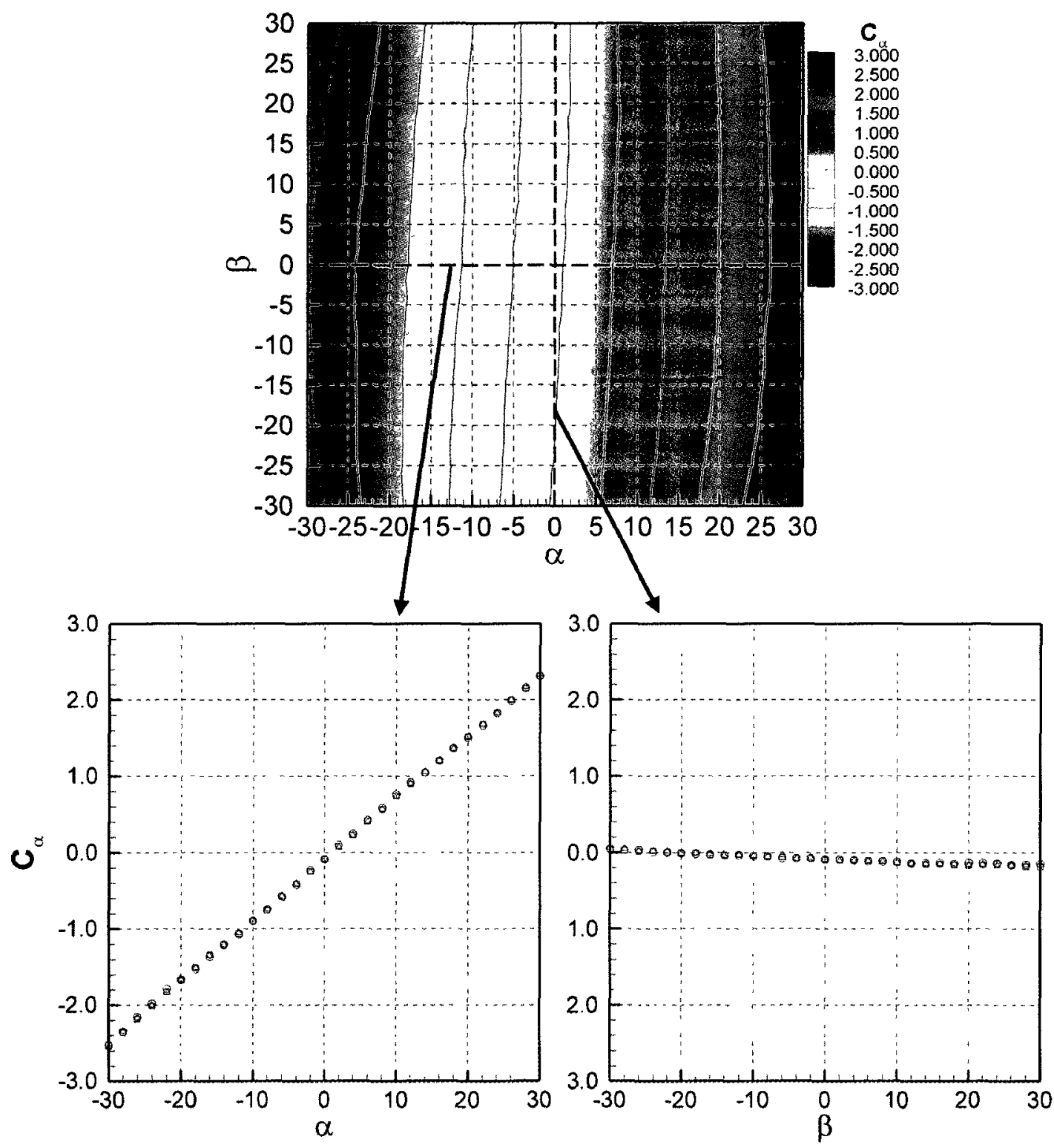

Figure 3.18: Sample Yaw Coefficient Calibration Surface for the Seven-Hole Pressure Probe (7HP1) 


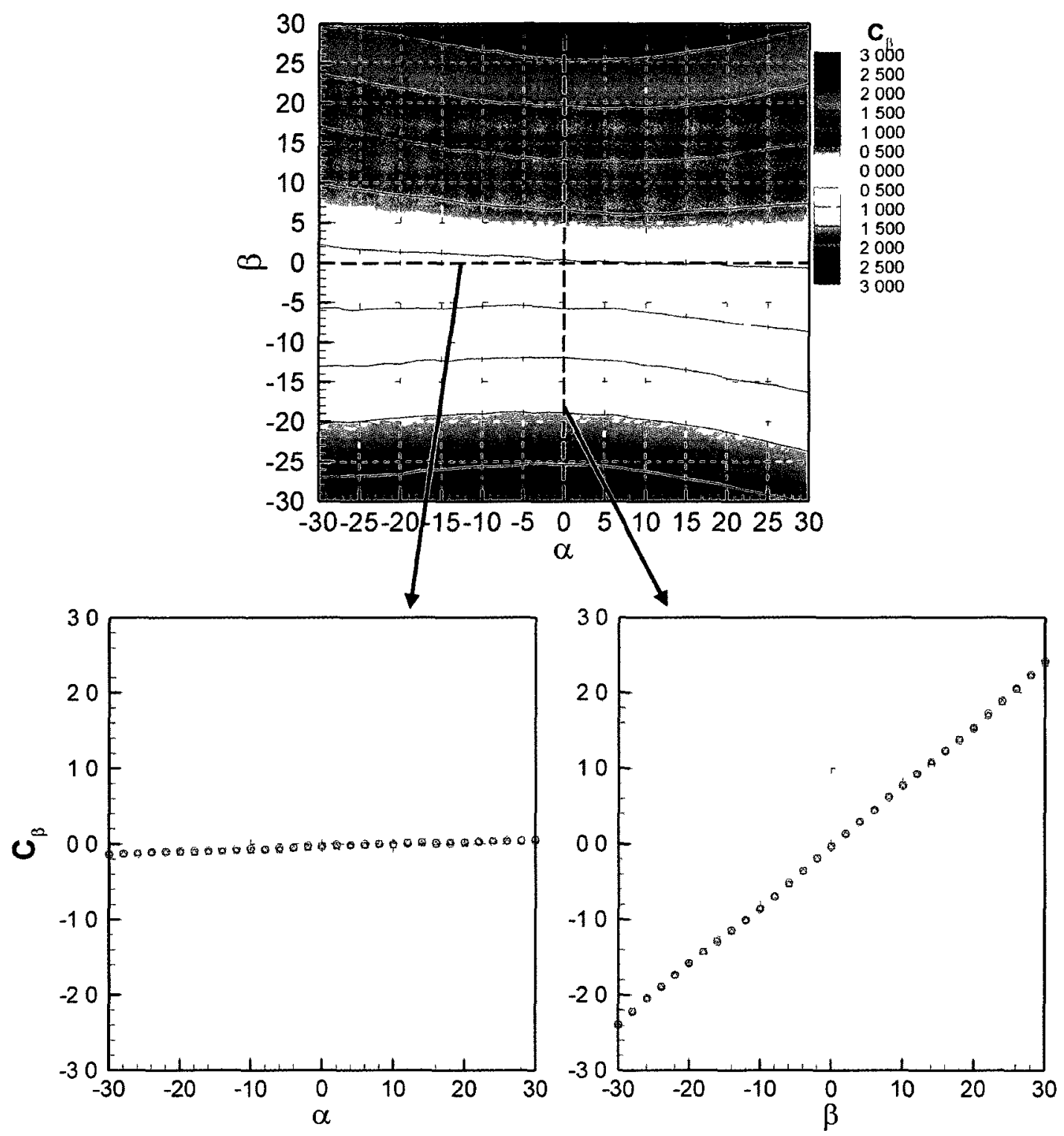

Figure 3.19: Sample Pitch Coefficient Calibration Surface for the Seven-Hole Pressure Probe (7HP1) 


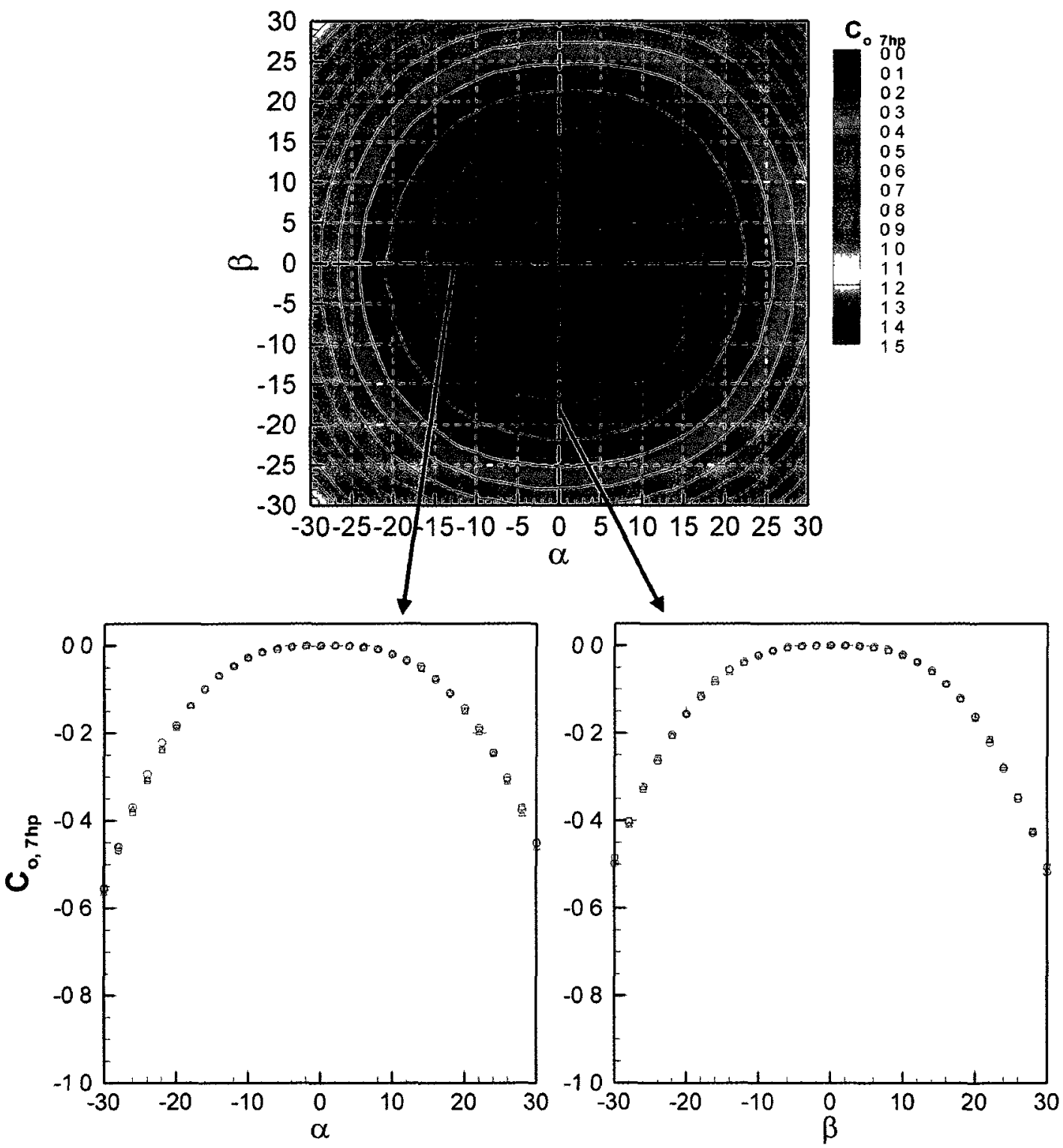

Figure 3.20: Sample Total Pressure Coefficient Calibration Surface for the Seven-Hole Pressure Probe (7HP1) 


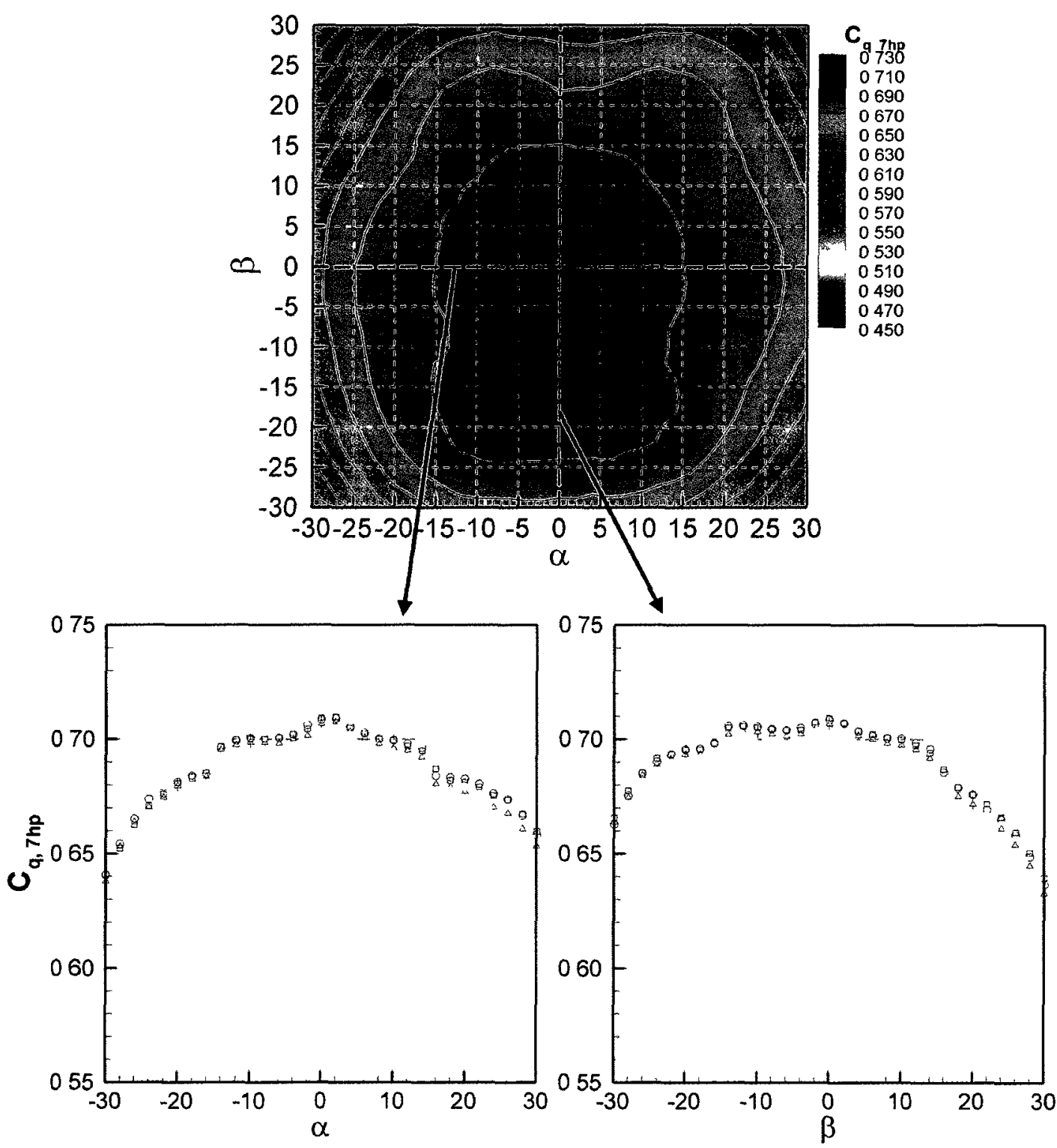

Figure 3.21: Sample Dynamic Pressure Calibration Surface for the Seven-Hole Pressure Probe (7HP1) 
For data reduction, $C_{\alpha}$ and $C_{\beta}$ are calculated at each data point from the measured port pressures. $\alpha$ and $\beta$ are inferred through bi-linear interpolation of Figures 3.18 and 3.19. Figures 3.20 and 3.21 are then interpolated using the flow angle to determine the values of $C_{P o 7 h p}$ and $C_{q 7 h p}$. Finally, the value of total pressure is calculated from $C_{P o 7 h p}$ and the value of dynamic pressure from $C_{q 7 h p}$.

Table 3.4 shows the experimental uncertainty in the seven-hole probe measurements here for all flow conditions. These uncertainties were determined using the same method as described for the three-hole probe. All data were collected using the same sample times as the three-hole probe (listed in Table 3.3).

\begin{tabular}{|c|c|c|c|c|c|}
\cline { 2 - 6 } \multicolumn{1}{c|}{} & \multicolumn{5}{c|}{ Uncertainty (all Reynolds numbers) } \\
\cline { 2 - 6 } \multicolumn{1}{c|}{} & $T u=1.5 \%$ & $T u=3 \%$ & $T u=5 \%$ & $T u=8 \%$ & $T u=12 \%$ \\
\hline $\begin{array}{c}\text { Total Pressure } \\
\text { (\% of true dynamic pressure) }\end{array}$ & \pm 0.5 & \pm 0.5 & \pm 0.5 & \pm 0.8 & \pm 1.0 \\
\hline $\begin{array}{c}\text { Dynamic Pressure } \\
(\% \text { of true dynamic pressure) }\end{array}$ & \pm 0.7 & \pm 0.7 & \pm 0.7 & \pm 0.9 & \pm 1.1 \\
\hline Flow Angle $\left({ }^{\circ}\right)$ & \pm 0.6 & \pm 0.6 & \pm 0.8 & \pm 0.8 & \pm 1.2 \\
\hline
\end{tabular}

Table 3.4: Seven-Hole Pressure Probe Measurement Uncertainty 


\title{
Chapter 4
}

\section{The Effect of Turbulence Intensity and}

\author{
Reynolds Number on the Aerodynamic
}

\section{Behaviour of Kiel, Three-Hole, and Seven-}

\section{Hole Pressure Probes}

\subsection{Introduction}

This chapter describes the modifications made to the low-turbulence calibration rig for high-turbulence operation and the experimental results for the response of the Kiel, three-hole, and seven-hole pressure probes to turbulence intensity and Reynolds number. These results are then applied in a re-examination of the wind tunnel data from Maclsaac et al. (2009) to demonstrate the influence of turbulence intensity on typical results from a linear turbine cascade. Also included here is technique for data reduction that can account for the effects of turbulence. 


\subsection{High-Turbulence Probe Calibration Rig}

The present study modified the existing low-turbulence probe calibration rig to generate turbulent calibration flows. Figure 4.1 shows a schematic of the modified rig.

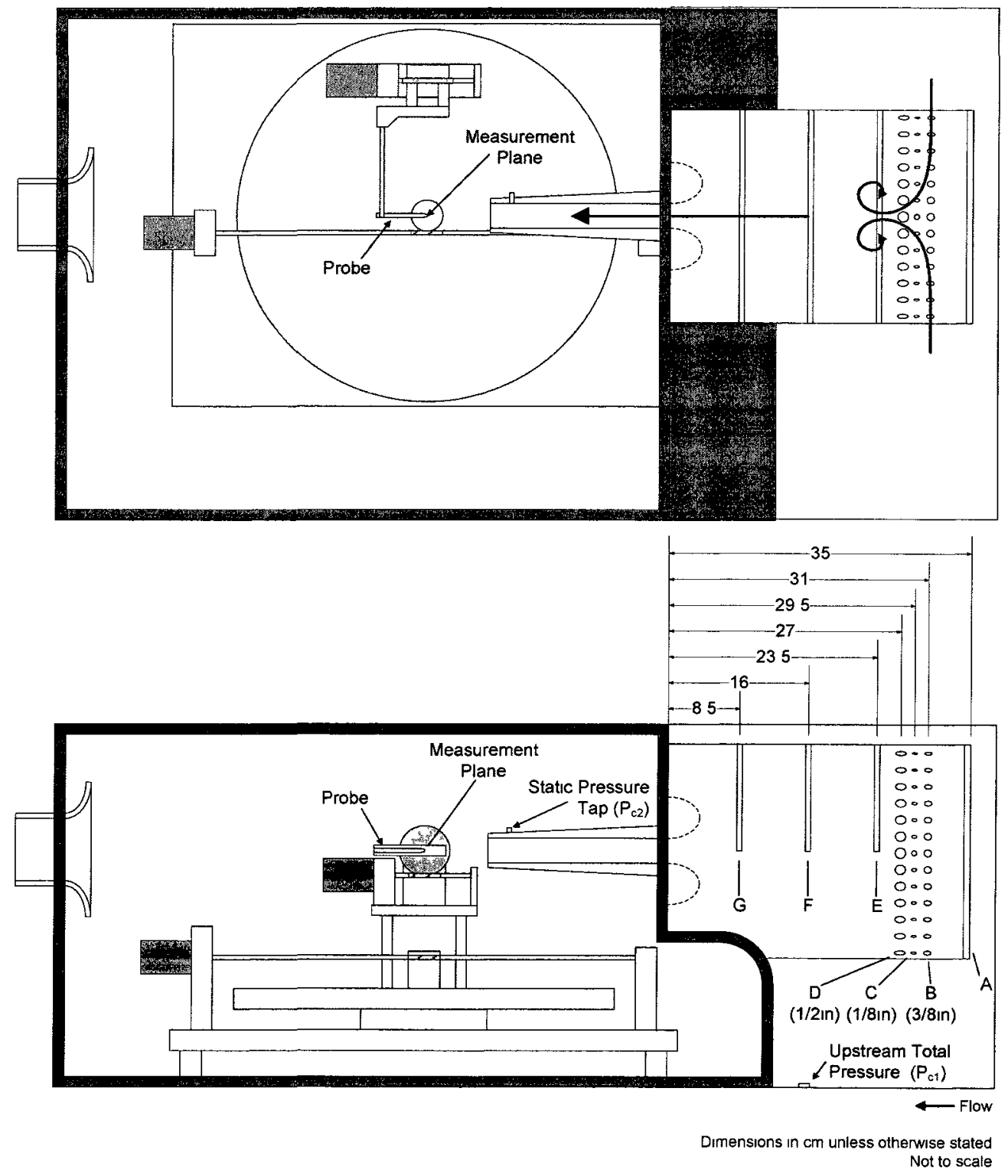

Figure 4.1: Schematic Diagram of the High-Turbulence Calibration Rig 
An inlet chamber made from 12 in diameter PVC pipe was added upstream of the bellmouth inlet of the original setup. This pipe had an upstream inlet (position A), three rows of circumferentially drilled holes (positions $\mathrm{B}, \mathrm{C}$, and $\mathrm{D}$, with hole diameters as indicated on the figure), and three slots (positions E, F, and G). The inlet, holes, and slots could all be sealed independently; when the inlet was open and all holes and slots were sealed the difference between the flows from the low- and high-turbulence rigs was negligible. This design allowed for the generation of turbulent flows through three mechanisms: (a) the use of one or more turbulence generating grids fixed to the inlet at $\mathrm{A}$ or inserted into slots $E, F$, or G, (b) mixing caused by the impinging jets from the circumferential holes (shown schematically in red in Figure 4.1), or (c) a combination of grids and mixing jets. Turbulence intensity was controlled by changing the grids and holes used; for a given combination of grids and holes, turbulence intensity was found to be nearly constant for all operating velocities.

The present study used several combinations of grids and holes to generate the turbulent flows examined here. Table 4.1 lists the primary arrangements employed. In this table, the term " $1 / 2$ open" indicates that alternating holes in that row were sealed and the term " $1 / 4$ open" indicates that three of every four holes were sealed. It should be noted that all setups shown in this table have the slots at E, F, and G sealed and without grids. These slots were unused for all data collection because it was found that multiple grids increased pressure losses, and thus decreased maximum operating velocities, without any appreciable increase in turbulence intensity. For any setup with two or more grids, the 
grid closest to the probe was dominant and the turbulence intensity and length scale generated by that setup was nearly identical to that which would be generated by the dominant grid on its own.

\begin{tabular}{|c|c|c|c|c|c|c|c|c|c|c|}
\hline \multirow[b]{2}{*}{$\begin{array}{c}\text { Setup } \\
\text { Number }\end{array}$} & \multirow{2}{*}{$\begin{array}{c}\text { Inlet } \\
\mathrm{A}\end{array}$} & \multicolumn{3}{|c|}{ Hole Rows } & \multicolumn{6}{|c|}{ Flow Properties at Measurement Plane } \\
\hline & & $\begin{array}{c}\text { B } \\
(3 / 8 \text { in })\end{array}$ & $\begin{array}{c}C \\
(1 / 8 \text { in })\end{array}$ & $\begin{array}{c}\mathrm{D} \\
(1 / 2 \mathrm{in})\end{array}$ & $\begin{array}{c}V \\
(\mathrm{~m} / \mathrm{s})\end{array}$ & $\begin{array}{c}T u \\
(\%)\end{array}$ & $\begin{array}{l}T u_{u} \\
(\%)\end{array}$ & $\begin{array}{l}T u_{v} \\
(\%)\end{array}$ & $\begin{array}{l}T u_{w} \\
(\%)\end{array}$ & $\begin{array}{c}\Lambda_{x} \\
(\mathrm{~mm})\end{array}$ \\
\hline \multirow{2}{*}{1} & Open & Sealed & Sealed & Sealed & 20 & 1.7 & 0.6 & 2.5 & 1.3 & - \\
\hline & Open & Sealed & Sealed & Sealed & 42 & 1.7 & 0.7 & 2.4 & 1.6 & - \\
\hline \multirow{2}{*}{2} & Open & Open & Open & $1 / 4$ Open & 21 & 2.7 & 2.0 & 3.9 & 3.1 & 7.2 \\
\hline & Open & Open & Open & $1 / 4$ Open & 41 & 3.1 & 2.0 & 3.2 & 2.7 & 4.6 \\
\hline \multirow[b]{2}{*}{3} & $\begin{array}{c}1 / 2 \text { in holes lin } \\
\text { apart }\end{array}$ & Sealed & Sealed & $1 / 4$ Open & 20 & 5.3 & 4.0 & 5.6 & 6.0 & 4.6 \\
\hline & $\begin{array}{c}1 / 2 \text { in holes } 1 \text { in } \\
\text { apart }\end{array}$ & Sealed & Sealed & $1 / 4$ Open & 39 & 4.8 & 3.6 & 5.3 & 5.4 & 4.0 \\
\hline \multirow{2}{*}{4} & Sealed & $1 / 2$ Open & Sealed & $1 / 2$ Open & 21 & 7.6 & 5.7 & 8.4 & 8.6 & 5.4 \\
\hline & Sealed & $1 / 2$ Open & Sealed & $1 / 2$ Open & 33 & 8.1 & 5.7 & 9.0 & 9.2 & 5.3 \\
\hline \multirow{2}{*}{5} & Sealed & Open & Sealed & Sealed & 21 & 12.2 & 7.3 & 14.3 & 13.7 & 7.4 \\
\hline & Sealed & Open & Sealed & Sealed & 32 & 12.5 & 7.3 & 14.7 & 14.0 & 7.5 \\
\hline
\end{tabular}

Table 4.1: High-Turbulence Probe Calibration Rig Hole and Grid Combinations

As can be seen from Table 4.1, the turbulence in all the flows examined here was anisotropic: crosswise turbulence intensities $\left(T u_{v}\right.$ and $\left.T u_{w}\right)$ were much greater than the streamwise component $\left(T u_{u}\right)$ for all cases. These values relate to the resultant turbulence intensity $(T u)$ through:

$$
\begin{aligned}
& T u_{u}=\frac{\sqrt{\overline{u^{\prime 2}}}}{\overline{\mathrm{V}}} \times 100 \\
& T u_{v}=\frac{\sqrt{\overline{v^{\prime 2}}}}{\overline{\mathrm{V}}} \times 100
\end{aligned}
$$




$$
\begin{gathered}
T u_{w}=\frac{\sqrt{\overline{w^{\prime 2}}}}{\bar{V}} \times 100 \\
T u=\frac{\sqrt{\frac{u^{\prime 2}+\overline{v^{\prime 2}}+\overline{w^{\prime 2}}}{3}}}{\sqrt{U^{2}+V^{2}+W^{2}}} \times 100
\end{gathered}
$$

where $U, V$, and $W$ are the mean velocities and $u^{\prime}, v^{\prime}$, and $w^{\prime}$ are their fluctuating components. The most plausible cause of this anisotropy in the turbulence is the accelerating nozzle located between the inlet chamber and the measurement plane. As summarized by Ertunc and Durst (2008), flow accelerations through nozzles are known to elongate the turbulent structures, decrease the streamwise component of turbulence, and increase the crosswise components. The effect this anisotropy had on the results presented in the following sections is unknown, but comparisons between results at different operating points in the current study are not expected to be greatly affected because the ratio of crosswise to streamwise turbulence intensities was relatively constant throughout the testing. Given the observed anisotropy, the reader should note that most discussion in the following sections is presented using the resultant turbulence intensity (Tu). Some discussion however is also included using the streamwise turbulence intensity $\left(T u_{u}\right)$ for readers interested in that relation.

To ensure that there were no spacial gradients in the calibration jet at the measurement plane, the total pressure, velocity, and streamwise turbulence intensity in the flow were evaluated over a distance of $\pm 6 \mathrm{~mm}$ from the centreline of the jet. Figures 
4.2 through 4.4 show results from this testing along the horizontal cut-plane for a typical operating point; similar measurements were taken for all operating points along multiple cut-planes but are omitted here because results were comparable to those shown. Total pressure is presented as the difference relative to the total pressure at the centre of the jet, $r=0$, and is normalized by centreline dynamic pressure. Velocity is given normalized by centreline velocity. The variation in turbulence intensity across the jet was evaluated using the streamwise component rather than the resultant turbulence intensity because it was relatively difficult to traverse the $\mathrm{X}$-wire hot-wire probe across the jet. Variations in the resultant and streamwise turbulence intensities across the jet were expected to be comparable. Given that the probes examined here are 1.37 to $2.4 \mathrm{~mm}$ in diameter, the

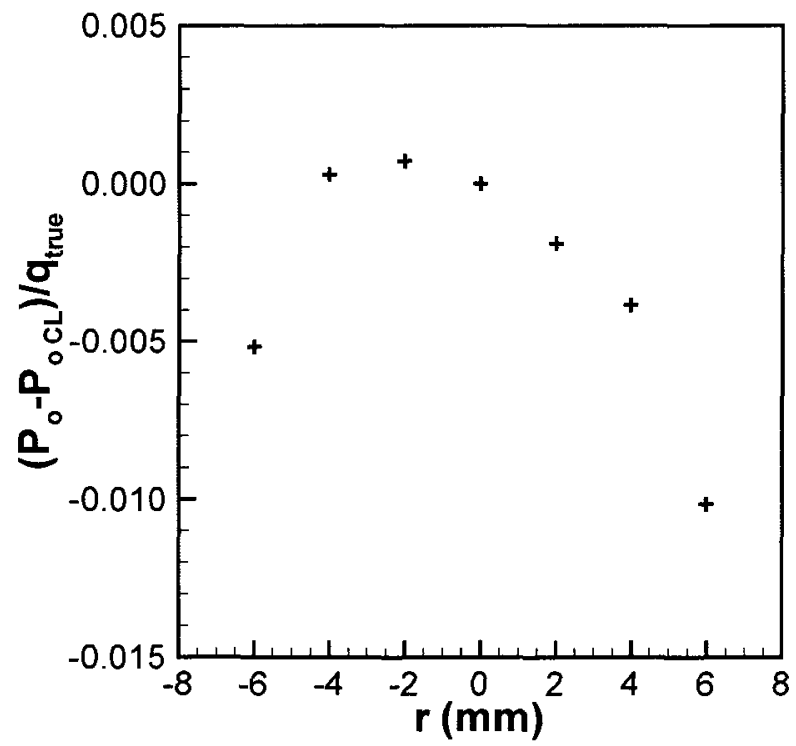

Figure 4.2: Profile of Total Pressure across the Calibration Jet at an Elevated Turbulence Intensity 


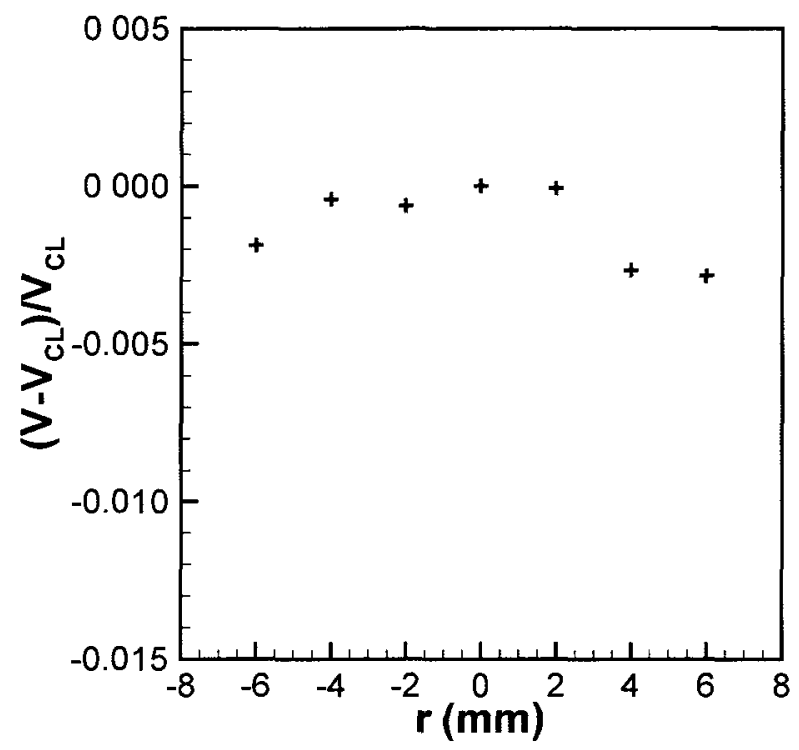

Figure 4.3: Profile of Velocity across the Calibration Jet at an Elevated Turbulence Intensity

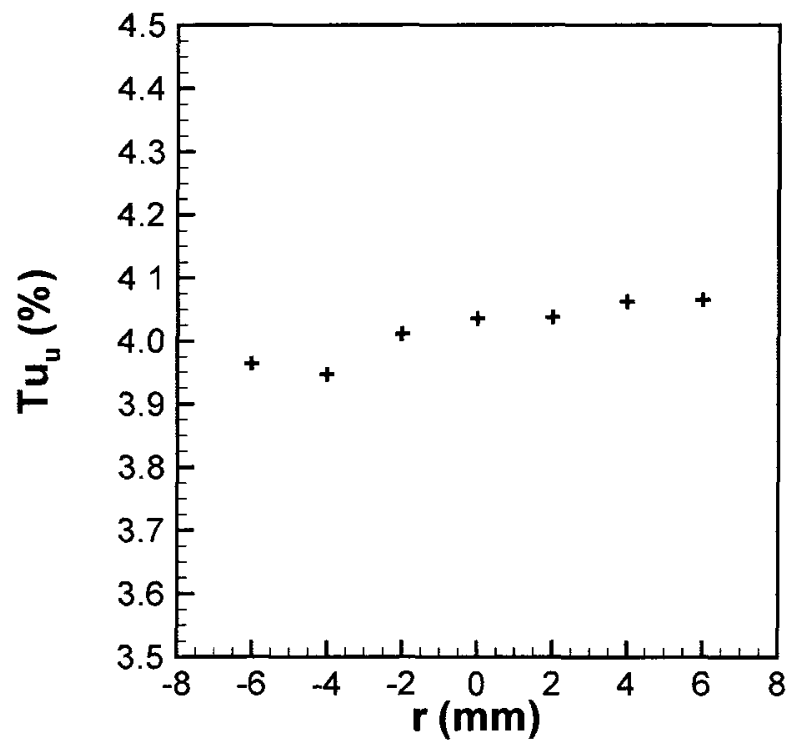

Figure 4.4: Profile of Streamwise Turbulence Intensity across the Calibration Jet at an Elevated Turbulence Intensity 
slight lateral variations in flow properties shown in the figures are expected to have negligible effects on results.

To the knowledge of the author, the use of impinging jets to generate turbulence for probe calibration is novel; most examples of turbulence generation in the open literature use turbulence generating grids. To ensure both grids and jets produced equivalent flows, the present study compared both methods at several turbulence intensities and Reynolds numbers. Results showed that the isotropy and length scales of turbulence produced by both methods were comparable at the measurement plane. The only notable difference between the turbulence generation methods was their pressure loss: losses from impinging jets were much less than those from grids when generating flows at a similar turbulence intensity and velocity. For this reason, jet generated turbulence was used almost exclusively in the current study.

\subsection{Effects of Turbulence Intensity and Reynolds Number on Flow Angle Sensitivity of the Kiel Probe}

Total pressure measurements were taken using the Kiel probe for the operating points listed in Table 4.2. Data were collected at each operating point over flow angles of $\pm 40^{\circ}$ in $2^{\circ}$ increments. 


\begin{tabular}{|cc|cc|c|c|c|c|}
\hline \multicolumn{2}{|c|}{$\begin{array}{c}\mathrm{Re}_{\mathrm{D}} \\
\text { [nominal] }\end{array}$} & \multicolumn{2}{|c|}{$\begin{array}{c}T u(\%) \\
\text { [nominal] }\end{array}$} & $\begin{array}{c}T u_{u} \\
(\%)\end{array}$ & $\begin{array}{c}T u_{v} \\
(\%)\end{array}$ & $\begin{array}{c}T u_{w} \\
(\%)\end{array}$ & $\begin{array}{c}\Lambda_{x} \\
(\mathrm{~mm})\end{array}$ \\
\hline 1060 & {$[1050]$} & 1.3 & {$[1.5]$} & 0.6 & 1.7 & 1.6 & - \\
\hline 1600 & {$[1600]$} & 1.3 & {$[1.5]$} & 0.6 & 1.7 & 1.6 & - \\
\hline 2180 & {$[2200]$} & 1.7 & {$[1.5]$} & 0.6 & 2.5 & 1.3 & - \\
\hline 2980 & {$[3000]$} & 1.7 & {$[1.5]$} & 0.6 & 2.3 & 1.7 & - \\
\hline 3640 & {$[3650]$} & 1.8 & {$[1.5]$} & 0.6 & 2.2 & 2.1 & - \\
\hline 4340 & {$[4350]$} & 1.7 & {$[1.5]$} & 0.7 & 2.4 & 1.6 & - \\
\hline 3000 & {$[3000]$} & 2.9 & {$[3]$} & 2.1 & 3.5 & 3.0 & 4.6 \\
\hline 2170 & {$[2200]$} & 5.3 & {$[5]$} & 4.0 & 5.6 & 3.1 & 4.6 \\
\hline 2960 & {$[3000]$} & 5.1 & {$[5]$} & 3.9 & 5.5 & 3.0 & 4.2 \\
\hline 2960 & {$[3000]$} & 7.9 & {$[8]$} & 5.6 & 8.7 & 8.9 & 5.3 \\
\hline 2150 & {$[2200]$} & 12.2 & {$[12]$} & 7.3 & 14.3 & 13.7 & 7.4 \\
\hline 2970 & {$[3000]$} & 12.4 & {$[12]$} & 7.3 & 14.7 & 14.0 & 7.5 \\
\hline
\end{tabular}

Table 4.2: Kiel Probe Test Matrix

Discussion below refers to each operating point using the nominal Reynolds numbers and resultant turbulence intensities included in brackets in Table 4.2. Nominal Reynolds numbers were chosen by rounding to the nearest 50 for each data set. Nominal turbulence intensities were chosen by rounding the mean of the resultant turbulence intensity for each inlet chamber configuration to the nearest $0.5 \%$. For example, data sets collected at a nominal $12 \%$ turbulence intensity were all obtained using inlet chamber setup number 5 in Table 4.1; this configuration produced turbulence intensities ranging from $11.8 \%$ to $12.4 \%$ depending on operating velocity.

The components of turbulence intensity for each operating point are notably anisotropic. The most likely cause of this anisotropy is the accelerating nozzle as discussed in Section 4.2. The effect this had on the results presented here is unknown, but comparisons between results at different operating points in the current study are not 
expected to be greatly affected because the ratio of crosswise to streamwise turbulence intensities was relatively constant throughout the testing.

The integral length scale of turbulence, $\Lambda_{x}$, represents the size of the dominant or most energetic turbulent structures in a flow. It was not possible in the present study to control the integral length scale of the turbulence independent of Reynolds number and turbulence intensity. Thus, the size of the eddies in the flow varied between the operating points examined. Eddy size is known to influence pressure probe measurements when the length scale is comparable to the diameter of the probe: turbulent structures of similar size to the probe can result in large local gradients in flow properties across the probe tip. As eddy size increases relative to the probe diameter, the influence of length scale decreases because the effective gradient over the probe becomes small. Although the variation in length scale between operating points seen here may have an influence on the results discussed; that influence is expected to be small as the dominant eddies observed are relatively large compared to the diameter of the Kiel probe $\left(\Lambda_{x} / D \approx 2.6\right.$ to 4.7$)$. It is recognized, however, that the full spectrum of turbulent structures likely includes some eddies that are of a comparable size to the probe tip.

Results are discussed below using the non-dimensional pressure coefficient, $C_{P_{0} \text { Kiel }}$, defined by:

$$
C_{P o \text { Kiel }}=\frac{P_{K i e l}-P_{c 2}}{q_{\text {true }}}
$$


where $P_{K i e l}$ is the pressure measured by the probe and $P_{c 2}$ is the reference nozzle static pressure. The effect of flow angularity is expressed as a change in $C_{P_{0} \text { Kiel }}$ as follows:

$$
\Delta C_{P o K i e l}=C_{P o K i e l}-C_{P o K t e l 0^{\circ}}
$$

where $C_{P o K i e l} 0^{\circ}$ is a reference value obtained by averaging the measured values of $C_{P o \text { Ktel }}$ over flow angles of $-10^{\circ} \leq \theta \leq 10^{\circ}$, a range for which the probe was essentially insensitive to flow angularity at all operating points. Figures below show $\Delta C_{P o K i e l}$ as a function of probe misalignment, $\theta$, and highlight the critical angle, $\theta_{c}$, defined as the flow angle at which misalignment effects are equal to one percent of dynamic pressure: that is, $\theta=\theta_{c}$ when $\Delta C_{P o K t e l}=-0.01$.

Figure 4.5 presents data collected for a turbulence intensity of $1.5 \%$ at three Reynolds numbers; data from three additional Reynolds numbers are included in Appendix B but omitted here as they show similar trends. These results demonstrate that Reynolds number effects on the flow angle sensitivity of the Kiel probe are minimal for $1050 \leq R e_{D} \leq 4350$ and $T u=1.5 \%$. The critical angle for all Reynolds numbers examined at this turbulence intensity was approximately $35^{\circ}$. Scatter in the $R e_{D}=1050$ results is greater than that for the higher Reynolds number results. This is due to poorer controllability in the operating point at lower Reynolds numbers. Figures 4.6 and 4.7 present results at turbulence intensities of $5 \%$ and $12 \%$, respectively, at two Reynolds numbers. These results suggest that the flow angle sensitivity of the Kiel probe is not a function of Reynolds number at any turbulence intensity. 


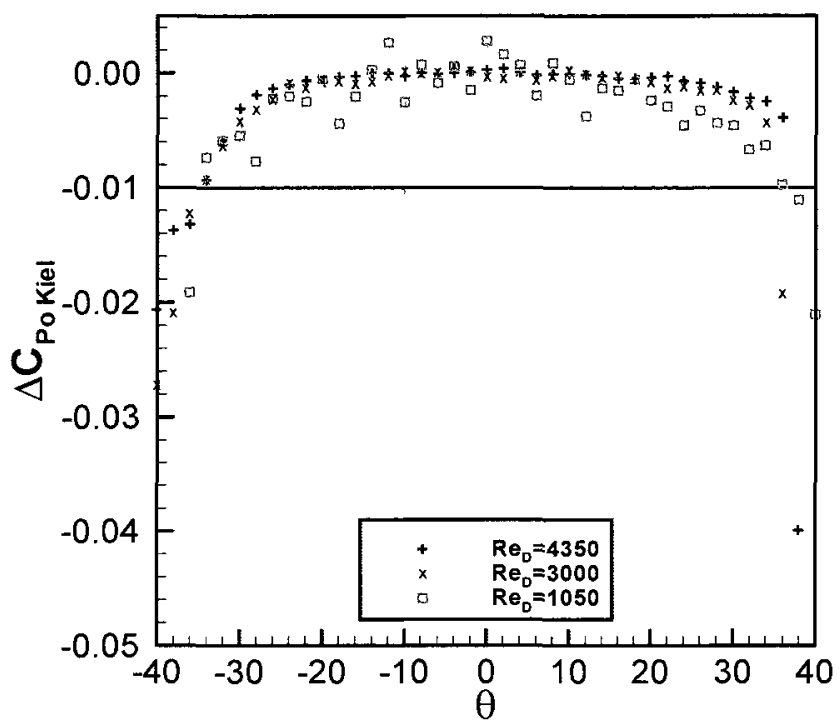

Figure 4.5: Kiel Probe Results for Total Pressure at Various Reynolds Numbers and $\mathrm{Tu}=\mathbf{1 . 5 \%}$

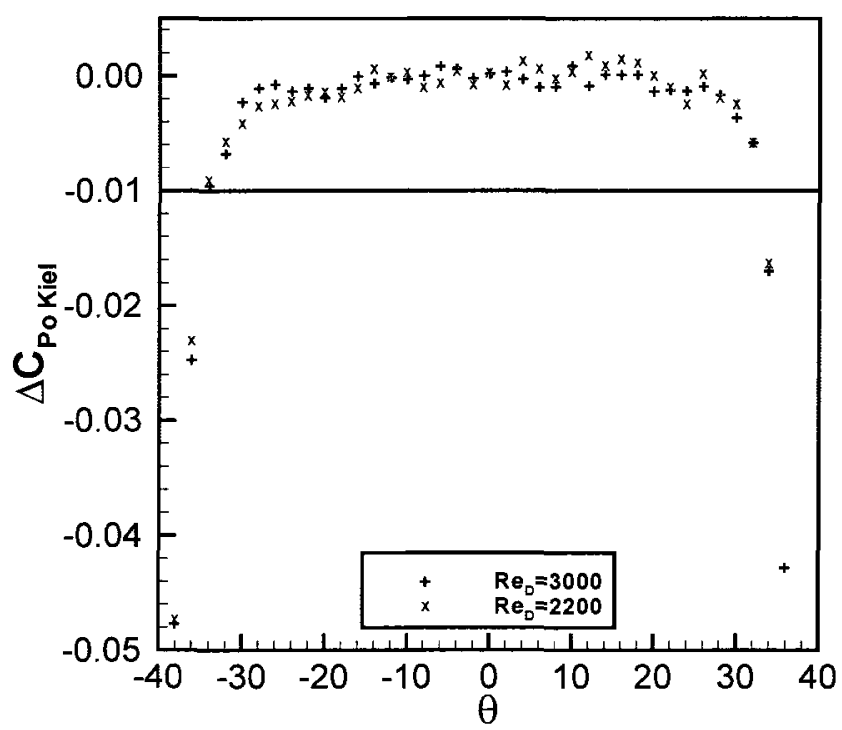

Figure 4.6: Kiel Probe Results for Total Pressure at Various Reynolds Numbers and $\mathbf{T u}=\mathbf{5 \%}$ 


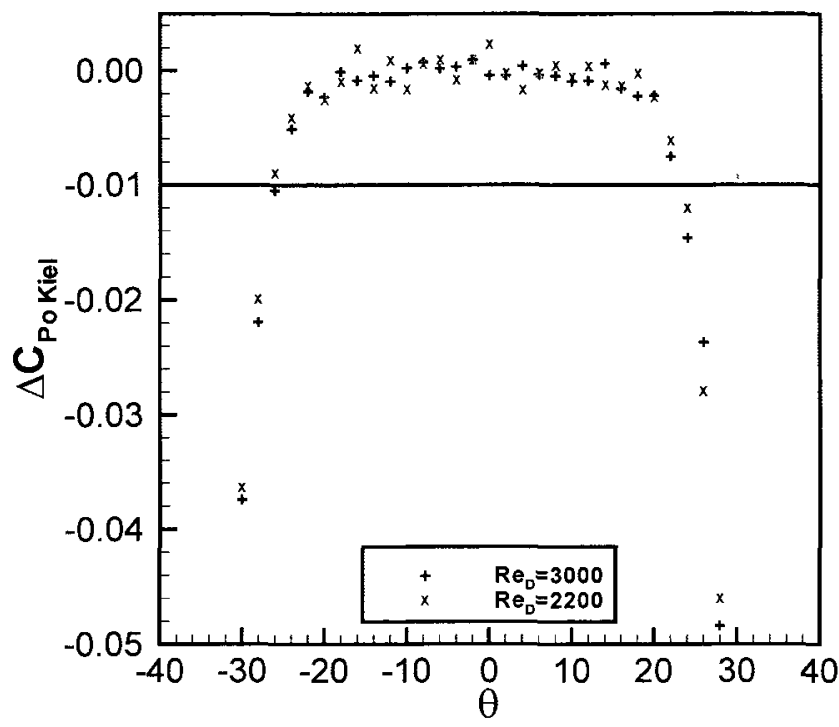

Figure 4.7: Kiel Probe Results for Total Pressure at Various Reynolds Numbers and $\mathrm{Tu}=\mathbf{1 2 \%}$

Figure 4.8 presents data collected at a Reynolds number of 3000 for five turbulence intensities. Figure 4.9 shows the critical angles for the measurements as a function of the resultant and streamwise turbulence intensities. The effect of turbulence intensity is small at low intensities but increases non-linearly as turbulence intensity increases. Critical angles for the Kiel probe here decreased from $35^{\circ}$ at $1.5 \%$ to $24^{\circ}$ at $12 \%$. These results are in agreement with similar results collected at a Reynolds number of 2200 , included in Appendix B, and demonstrate that the Kiel probe becomes more sensitive to flow misalignment as turbulence intensity increases. 


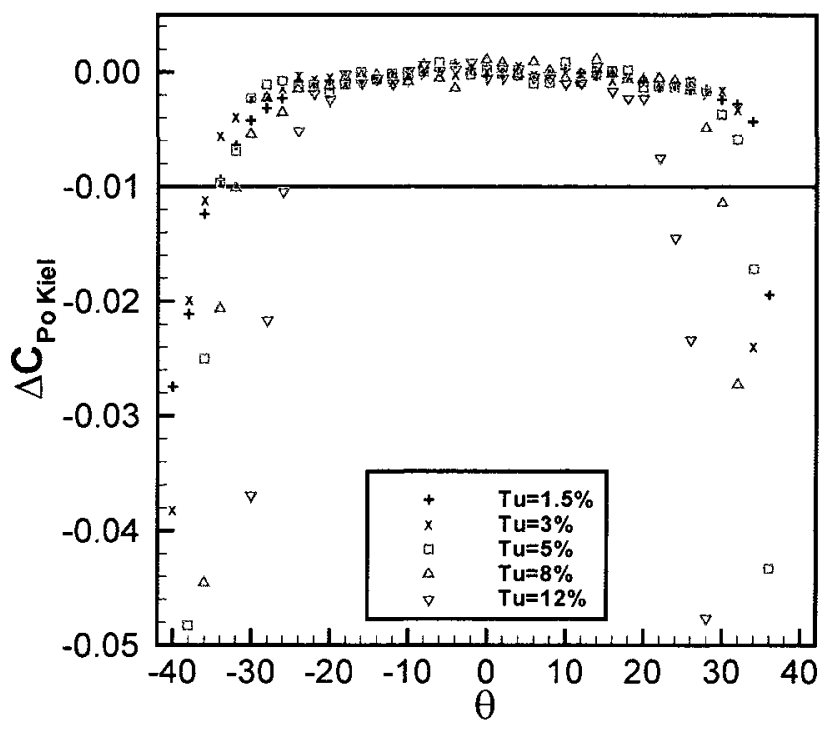

Figure 4.8: Kiel Probe Results for Total Pressure at $\mathbf{R e}_{\mathrm{D}}=\mathbf{3 0 0 0}$ and Various Turbulence Intensities

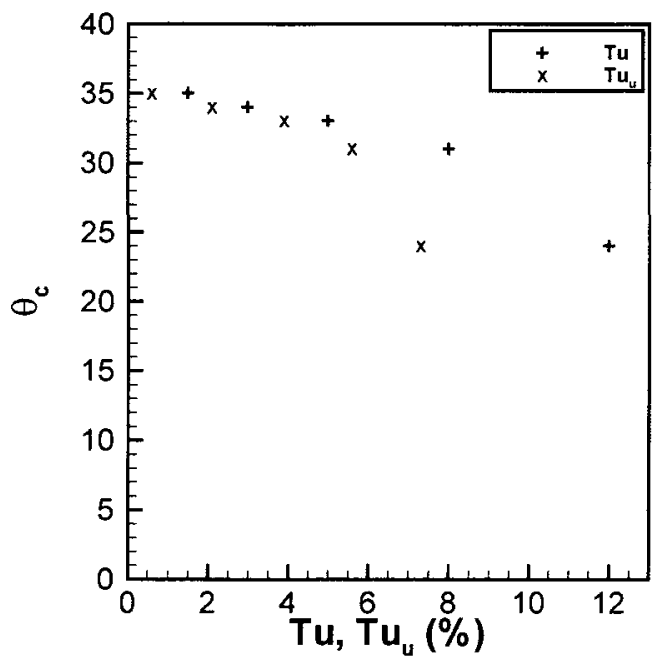

Figure 4.9: Kiel Probe Results for Critical Angle as a function of Turbulence Intensity 


\subsection{Effects of Turbulence Intensity and Reynolds Number on Three-Hole Pressure Probes}

\subsubsection{Introduction}

In the past, researchers using three-hole pressure probes to investigate highturbulence flows have typically calibrated their probes in low-turbulence flows. To the knowledge of the present author, the error introduced by this data reduction at "off-calibration" turbulence intensities has not been quantified in the open literature. Thus, the following section presents an estimate of this error. In addition, the error resulting from data reduction at off-calibration Reynolds numbers is also investigated. It should be noted however that while the turbulence intensity study presented here is believed to be novel, previous studies, such as that by Benner (2003), have investigated the effects of off-calibration Reynolds numbers.

The standard procedure for data collection using a multi-hole pressure probe is a twostep process: calibrate the probe in a known flow and then use this calibration to reduce data collected in a flow of interest. The current study applies this procedure with one notable difference: both the calibration data sets and the data of interest, referred to here as a "validation data sets", are collected in a known flow using the calibration rig. This means that the true values of total pressure, dynamic pressure, and flow angle are known for all validation data points. Thus, the error in any results can be determined by 
comparing the results inferred from the three-hole probe measurements to the true values. This comparison is presented using:

$$
\begin{gathered}
\Delta \alpha=\alpha_{\text {Inferred }}-\alpha_{\text {True }} \\
\Delta C_{P_{o}}=\frac{P_{o \text { Inferred }}-P_{o \text { True }}}{q_{\text {True }}} \\
\Delta C_{q}=\frac{q_{\text {Inferred }}-q_{\text {True }}}{q_{\text {True }}}
\end{gathered}
$$

where $\Delta C_{P o}$ and $\Delta C_{q}$ are the errors in total and dynamic pressure normalized by true dynamic pressure and $\Delta \alpha$ is the flow angle error in degrees.

Calibration and validation data sets were collected at the operating points listed in Table 4.3. Each calibration data set consisted of measurements at yaw misalignment angles of -30 to $30^{\circ}$ in $1^{\circ}$ steps. Validation data sets included measurements at -25.5 to $25.5^{\circ}$ in $1^{\circ}$ steps. To determine the effects of off-calibration data reduction on results, each calibration was used to reduce all validation data sets including those at offcalibration conditions.

Although the off-calibration results could be presented using any calibration, the main text details only one case for each of the turbulence intensity and Reynolds number discussions for brevity. These cases are chosen to represent the most likely scenarios encountered by the users of such probes. For turbulence intensity, off-calibration results 


\begin{tabular}{|c|c|c|c|c|c|}
\hline $\begin{array}{c}\operatorname{Re}_{\mathrm{D}} \\
\text { [nominal] }\end{array}$ & $\begin{array}{c}T u(\%) \\
\text { [nominal] }\end{array}$ & $\begin{array}{l}T u_{u} \\
(\%)\end{array}$ & $\begin{array}{l}T u_{v} \\
(\%)\end{array}$ & $\begin{array}{l}T u_{w} \\
(\%)\end{array}$ & $\begin{array}{c}\Lambda_{x} \\
(\mathrm{~mm})\end{array}$ \\
\hline $1840 \quad[1850]$ & $1.7 \quad[1.5]$ & 0.6 & 2.5 & 1.3 & - \\
\hline $2530[2500]$ & $1.7 \quad[1.5]$ & 0.6 & 2.3 & 1.7 & - \\
\hline $4100[4100]$ & $1.7 \quad[1.5]$ & 0.7 & 2.4 & 1.6 & - \\
\hline $1860[1850]$ & 3.1 & 2.0 & 3.9 & 3.1 & 7.2 \\
\hline $2530[2500]$ & 2.9 & 2.1 & 3.5 & 3.0 & 4.6 \\
\hline $1830[1850]$ & 5.3 & 4.0 & 5.6 & 6.0 & 4.6 \\
\hline $2510[2500]$ & 5.1 & 3.9 & 5.5 & 5.7 & 4.2 \\
\hline $3810[3800]$ & 4.8 & 3.6 & 5.3 & 5.4 & 4.0 \\
\hline $1850[1850]$ & 7.7 & 5.7 & 8.4 & 8.6 & 5.4 \\
\hline 2550 [2500] & 7.9 & 5.7 & 8.7 & 8.9 & 5.3 \\
\hline $1870[1850]$ & $12.2[12]$ & 7.3 & 14.3 & 13.7 & 7.4 \\
\hline $2570[2500]$ & $12.4[12]$ & 7.3 & 14.6 & 14.1 & 7.5 \\
\hline
\end{tabular}

Table 4.3: Three-Hole Pressure Probe Calibration and Validation Test Matrix

are discussed using the low-turbulence calibration $(T u=1.5 \%)$ to reduce data at all other turbulence intensities. For Reynolds number, results are discussed using a calibration collected at the highest Reynolds number $\left(R e_{D}=4100\right)$. Readers looking for quantitative results for the other cases should refer to Appendix C, but in general, the results given in the text describe the trends observed for all results and qualitative deductions can be made from them. For example, if reducing validation data at $T u=12 \%$ with a calibration at $T u=1.5 \%$ yields $\Delta C_{P o}=-0.05$, the reader can expect that reducing validation data at $12 \%$ using a calibration at $5 \%$ would result in $0>\Delta C_{P_{0}}>-0.05$ and reducing data at $1.5 \%$ using a calibration at $12 \%$ would yield $\Delta C_{P o} \approx+0.05$.

As discussed previously in Sections 4.2 and 4.3, the components of the turbulence intensities at each operating point are notably anisotropic and the length scale of the turbulence varies for each operating point investigated. The nature and magnitude of the 
effects that these factors had on the results presented here are unknown, but the effect of the length scale variation is expected to be small since the dominant eddy size at all operating points was larger than the diameter of the three-hole probe $\left(\Lambda_{x} / D \approx 2.9\right.$ to 5.5$)$. It is recognized, however, that smaller eddies are likely also present in the flow. Because of the anisotropy in the turbulence intensity, the reader should note that most discussion presented here uses the nominal values of the resultant turbulence intensity $(T u)$ included in brackets in Table 4.3; these nominal values have been assigned in the same way as in Section 4.3. Because it is recognized that past research has often been characterized by the streamwise turbulence intensity $\left(T u_{u}\right)$, some discussion is also included using this flow parameter.

\subsubsection{Effects of Turbulence Intensity on Three-Hole Pressure Probe Flow Measurements at Off-Calibration Conditions}

This section demonstrates the effects of reducing data at off-calibration turbulence intensities by using a calibration taken at a resultant turbulence intensity of $1.5 \%$ to reduce validation data collected at five intensities ranging from 1.5 to $12 \%$. The effects of this procedure on flow angle, total pressure, and dynamic pressure are discussed separately below. All data shown in the main text were collected at a Reynolds number of 2500; similar results were obtained at $R e_{D}=1850$ and are included in Appendix C.

Figure 4.10 shows results for flow angle from the validation data collected at $1.5 \%$. Figures 4.11 and 4.12 show results from reducing validation data at $5 \%$ and $12 \%$ using the same calibration. The on-calibration results in Figure 4.10 are as expected: the 
precision error in the measurements is within the estimated uncertainty of the experiment and there are no systematic errors observed. Figure 4.11 however shows a slight positive slope and results in Figure 4.12 show a sinusoidal variation in $\Delta \alpha$. Although omitted here, results for validation data taken at $8 \%$ were similar to the $12 \%$ results and thus neither appears to be a false trend in the measurements.

The cause of these flow angle results is not apparent from the data presented here. The following section however presents off-calibration Reynolds number results which also showed similar trends. The cause of these results is discussed in more detail in this next section, but in summary the sinusoidal variation may be related to flow separation on the tip of the probe.

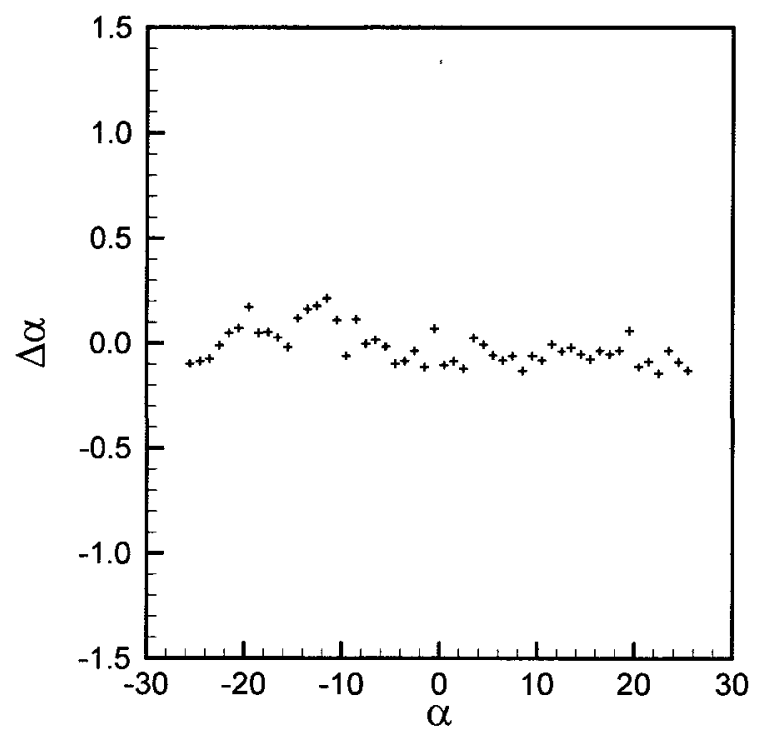

Figure 4.10: Three-Hole Pressure Probe Results for Flow Angle using Calibration and Validation Data at $\operatorname{Re}_{\mathrm{D}}=\mathbf{2 5 0 0}, \mathrm{Tu}=\mathbf{1 . 5 \%}$ 


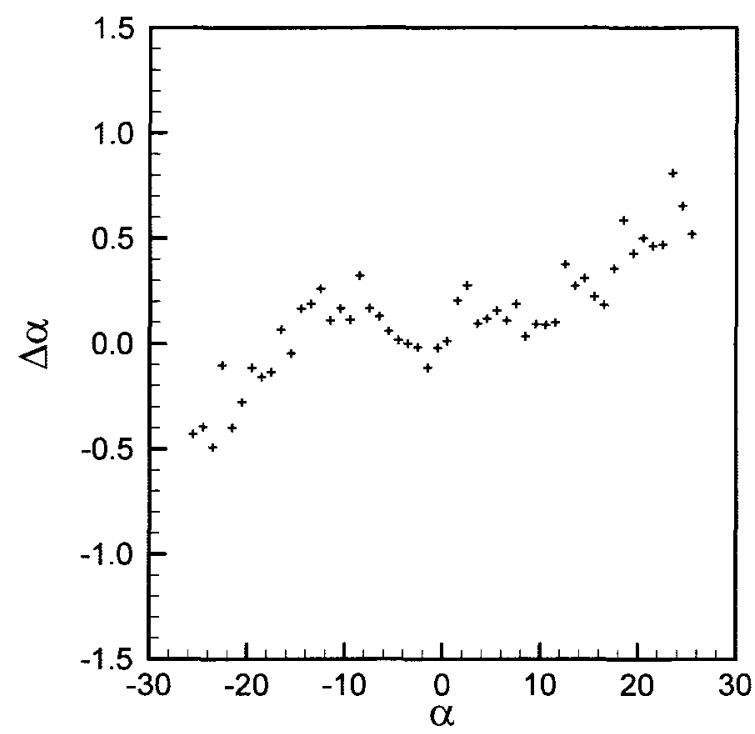

Figure 4.11: Three-Hole Pressure Probe Flow Angle Error due to Turbulence Intensity using a Calibration at $\operatorname{Re}_{\mathrm{D}}=\mathbf{2 5 0 0}, \mathrm{T} u=1.5 \%$ and Validation Data at $\operatorname{Re}_{\mathrm{D}}=\mathbf{2 5 0 0}, \mathrm{Tu}=5 \%$

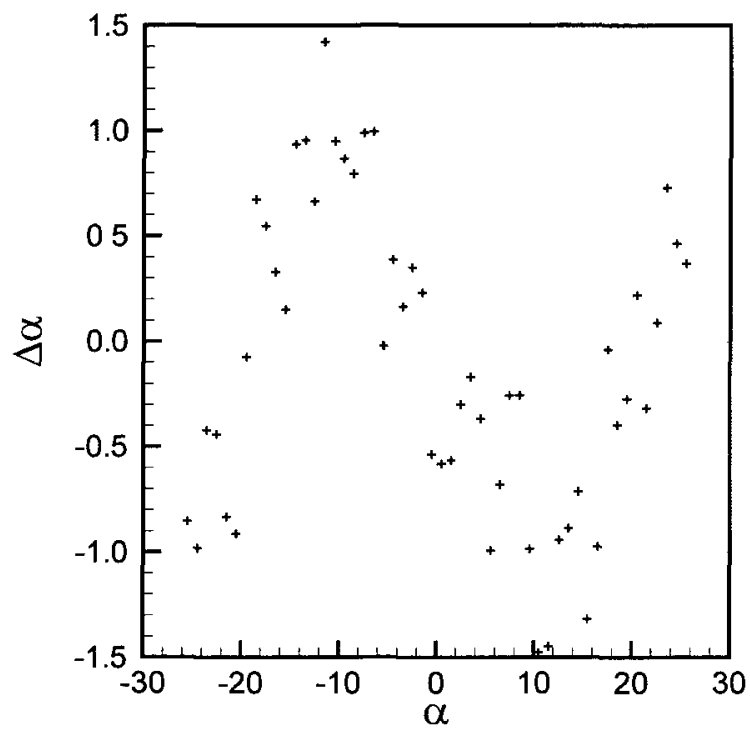

Figure 4.12: Three-Hole Pressure Probe Flow Angle Error due to Turbulence Intensity

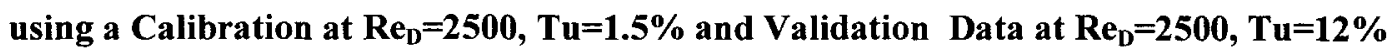


Figure 4.13 shows results for total pressure at off-calibration conditions. Results for validation data sets from five resultant turbulence intensities are shown in the figure; all data is reduced using the calibration at $1.5 \%$. These results show that reducing data at a high turbulence intensity using a low turbulence calibration results in a systematic error in total pressure for measurements away from $\alpha=0$. This underestimation of total pressure increases with the magnitude of flow angle; the maximum error in total pressure at the flow conditions observed here occurred at $\alpha \approx \pm 18^{\circ}$.

The errors in total pressure at positive flow angles were found to be greater than those at negative flow angles. The specific cause of this is unknown. One plausible explanation is asymmetry of the tip of the probe. Although probe tip asymmetry at on-calibration conditions is accounted for in the probe calibration, this asymmetry can

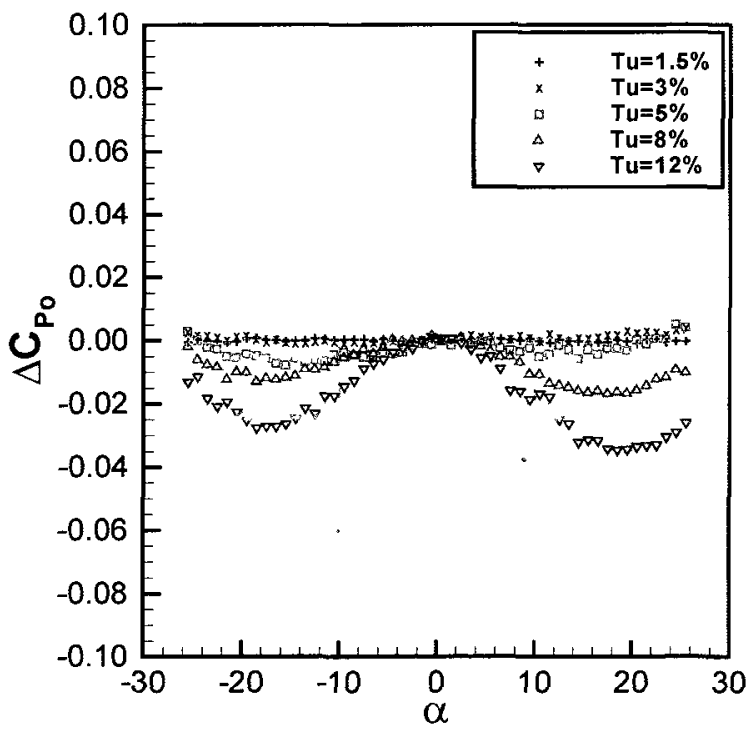

Figure 4.13: Three-Hole Pressure Probe Total Pressure Error due to Turbulence Intensity using a Calibration at $\operatorname{Re}_{\mathrm{D}}=\mathbf{2 5 0 0}, \mathrm{Tu}=\mathbf{1 . 5 \%}$ 
result in the two sides of the probe responding differently to changes in the flow. Thus, the effect of turbulence at positive flow angles may be different from that at negative flow angles, leading to the asymmetry seen here.

To summarize the results above, Figures 4.14 and 4.15 plot the root-mean-square (RMS) and maximum values of total pressure error (in this case, the minimum value of $\left.\Delta C_{P o}\right)$ versus resultant and streamwise turbulence intensities; approximate trend lines have also been included. The RMS values are defined as:

$$
\Delta C_{P o r m s}=\sqrt{\frac{\left(\Delta C_{P o 1}\right)^{2}+\left(\Delta C_{P o 2}\right)^{2}+\cdots+\left(\Delta C_{P o n}\right)^{2}}{n}}=\sqrt{\overline{\left(\Delta C_{P o}\right)^{2}}}
$$

These figures show that the systematic error in total pressure at off-calibration conditions increases non-linearly with turbulence intensity. Thus, calibrating at the correct turbulence intensity is more important when collecting data at higher turbulence intensities.

$\Delta C_{P o}=0$ at $\alpha=0^{\circ}$ for all off-calibration results shown above. This, however, is a product of the data reduction procedures and not necessarily the actual probe response. The parameter $\Delta C_{P o}$, previously defined as:

$$
\Delta C_{P_{o}}=\frac{P_{\text {oInferred }}-P_{\text {oTrue }}}{q_{\text {True }}}
$$




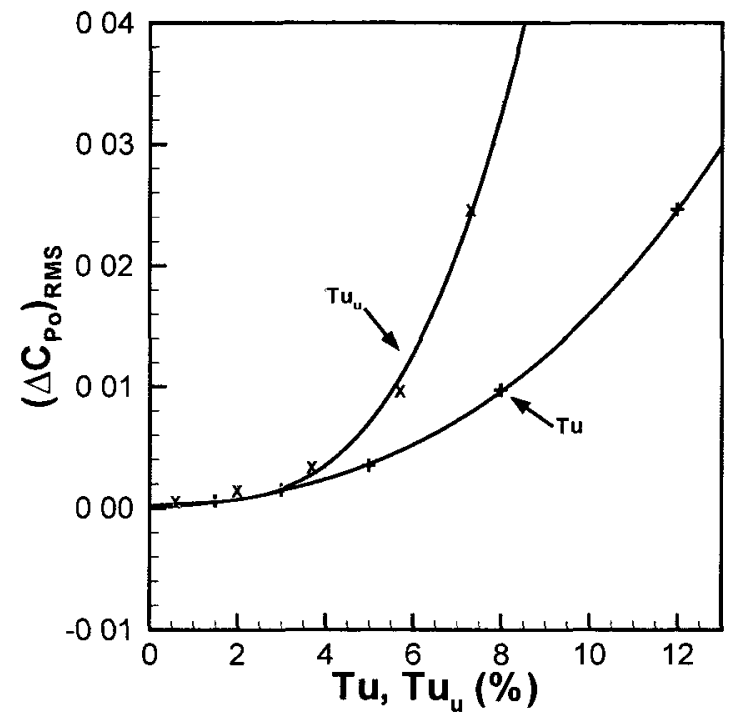

Figure 4.14: Three-Hole Pressure Probe RMS Total Pressure Error due to Turbulence Intensity using a Calibration at $\operatorname{Re}_{\mathrm{D}}=\mathbf{2 5 0 0}$ and $\mathrm{Tu}=\mathbf{1 . 5 \%}$

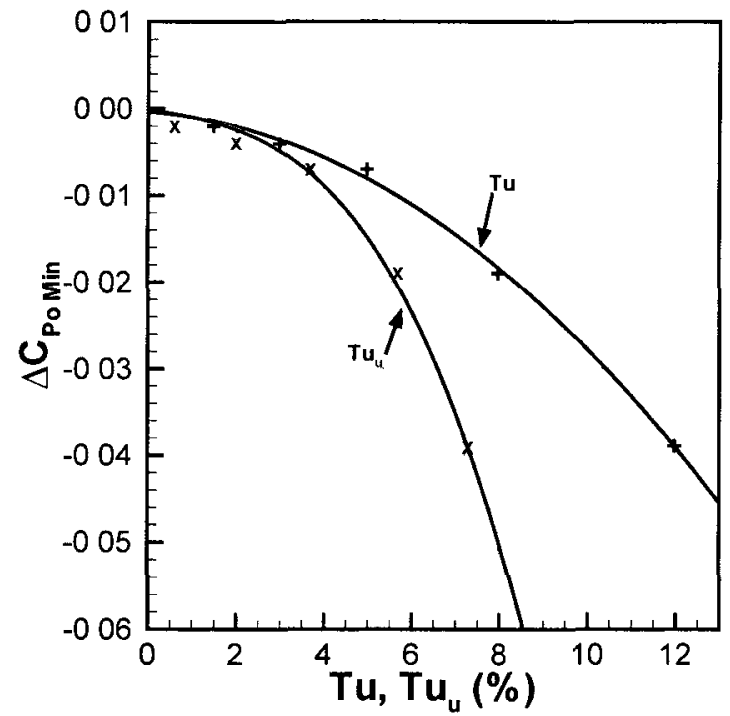

Figure 4.15: Three-Hole Pressure Probe Maximum Total Pressure Error due to Turbulence Intensity using a Calibration at $\mathrm{Re}_{\mathrm{D}}=\mathbf{2 5 0 0}$ and $\mathrm{Tu}=\mathbf{1 . 5 \%}$ 
is the difference between the total pressure inferred from the three-hole probe measurements and the true total pressure. True total pressure was obtained using the coefficient $C_{P o C a l}$ defined previously by Equation 3.1 as:

$$
C_{P o \text { Cal }}=\frac{P_{o \text { True }}-P_{c 1}}{P_{c 1}-P_{c 2}}
$$

Prior to collecting calibration or validation data, $C_{P o C a l}$ was determined by measuring the value of total pressure at the measurement plane for each operating point. These measurements were taken using the centre port of the three-hole probe which behaves essentially as a Pitot probe when aligned with the flow. The by-product of this procedure is that the values for true total pressure include any systematic error present in the aligned three-hole probe values.

As discussed in Section 2.2.6, several researchers have shown that Pitot probes tend to overestimate total pressure in turbulent flows. Although no research states it explicitly, it is likely that the centre port of the three-hole probe will similarly overestimate total pressure in turbulence. As such, the true total pressure values used here may be overestimated for high-turbulence operating points. Thus, results presented in Figure 4.13 include a bias error in $\Delta C_{P_{0}}$ that is constant across all flow angles for a given turbulence intensity. 
A simple estimate of the bias due to turbulence can be developed using the work of Goldstein (1936) which states that the total pressure measured by a Pitot tube in turbulent flow overestimates total pressure according to:

$$
P_{\text {oMeasured }}=P_{o \text { True }}+\frac{1}{2} \rho \overline{\mathrm{V}^{\prime 2}}
$$

The influence of the fluctuating component can then be written as:

$$
\begin{aligned}
\Delta C_{\text {Po Bras }} & \leq-\frac{\frac{1}{2} \rho \overline{\mathrm{V}^{\prime 2}}}{q_{\text {True }}} \\
& \leq-\frac{\frac{1}{2} \rho \overline{\mathrm{V}^{\prime 2}}}{\frac{1}{2} \rho \mathrm{V}^{2}} \\
& \leq-(T u)^{2}
\end{aligned}
$$

Table 4.4 lists the estimate of this bias at each resultant turbulence intensity. It should be noted that the biases estimated for $T u \geq 8 \%$ are greater than the overall uncertainty quoted for total pressure measurements using the three-hole probe.

\begin{tabular}{|c|c|}
\hline$T u(\%)$ & $\Delta \mathrm{C}_{\text {PoBaas }}$ \\
\hline 1.5 & -0.0002 \\
\hline 3 & -0.0009 \\
\hline 5 & -0.0025 \\
\hline 8 & -0.0064 \\
\hline 12 & -0.014 \\
\hline
\end{tabular}

Table 4.4: Estimated Bias in Three-Hole Probe Total Pressure Results due to Turbulence 
Results for dynamic pressure at off-calibration conditions are shown in Figure 4.16. These results show that reducing high-turbulence data using a low-turbulence calibration results in a systematic error in dynamic pressure at most flow angles. $\Delta C_{q}$ was found to vary significantly over the flow angles investigated; maximum error in dynamic pressure occurred around $\alpha \approx \pm 18^{\circ}$.

Unlike the total pressure results previously discussed, off-calibration results for dynamic pressure do not all pass through $\Delta C_{q}=0$ at $\alpha=0$. This may be the genuine probe response to turbulence intensity, but it may also be the result of uncertainty in the true dynamic pressure. Results here are shown using the parameter $\Delta C_{q}$, previously defined as:

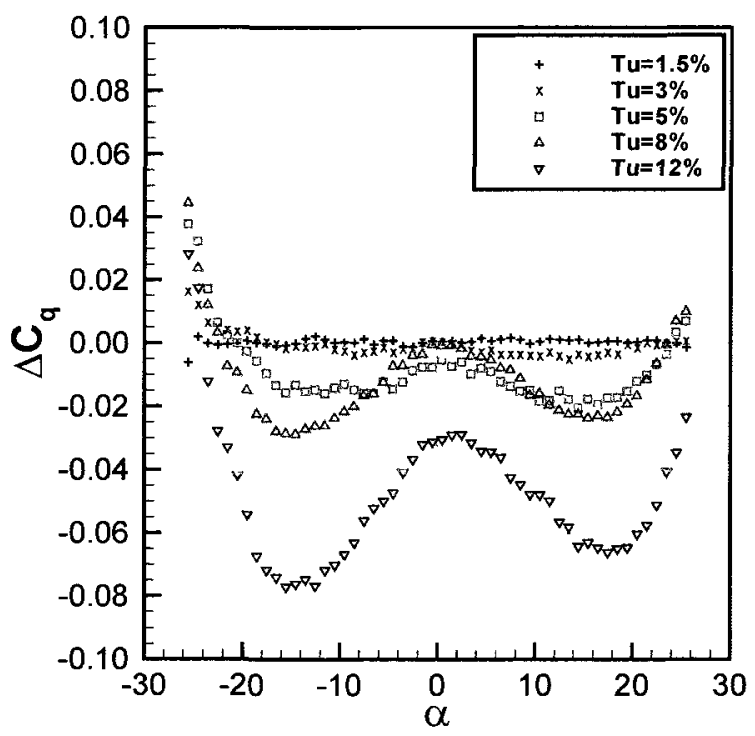

Figure 4.16: Three-Hole Pressure Probe Dynamic Pressure Error due to Turbulence Intensity using a Calibration at $\operatorname{Re}_{\mathrm{D}}=\mathbf{2 5 0 0 , T u = 1 . 5 \%}$ 


$$
\Delta C_{q}=\frac{q_{\text {Inferred }}-q_{\text {True }}}{q_{\text {True }}}
$$

This parameter compares the dynamic pressure inferred from the three-hole probe to the true dynamic pressure. Thus, uncertainty in $q_{\text {True }}$ directly influences the results shown here.

The value of true dynamic pressure was determined for each test using the coefficient $C_{q C a l}$ previously defined by:

$$
C_{q C a l}=\frac{q_{\text {True }}}{P_{c 1}-P_{c 2}}
$$

Prior to data collection, $C_{q \text { Cal }}$ was established for each operating point by finding $q_{\text {True }}$ at the measurement plane. For the operating points at turbulence intensities of $1.5 \%$ and $3 \%, q_{\text {True }}$ was obtained by measuring the total and static pressures in the jet. For all other operating points, $q_{\text {True }}$ was calculated using velocity measurements from the X-wire hotwire probe. Table 4.5 presents the values of $C_{q C a l}$ obtained here; the uncertainties quoted for $C_{q C a l}$ were estimated using the uncertainties in the contributing pressure or $\mathrm{X}$-wire measurements. The value of $C_{q C a l}$ was found to depend solely on the combination of grids and holes used to generate the turbulence intensity, irrespective of Reynolds number, thus $C_{q \mathrm{Cal}}$ is constant for each nominal turbulence intensity. 


\begin{tabular}{|c|c|}
\hline$T u(\%)$ & $\mathrm{C}_{\mathrm{qCal}}$ \\
\hline 1.5 & $0.992 \pm 0.005$ \\
\hline 3 & $0.987 \pm 0.010$ \\
\hline 5 & $0.932 \pm 0.047$ \\
\hline 8 & $0.581 \pm 0.029$ \\
\hline 12 & $0.452 \pm 0.023$ \\
\hline
\end{tabular}

Table 4.5: Values of $\mathrm{C}_{q \mathrm{Cal}}$ for Different Turbulence Intensities

Figure 4.17 shows the effect of this uncertainty in $C_{q \text { Cal }}$ using the calibration at $1.5 \%$ to reduce the validation data taken at $12 \%$. The validation data in this figure have been reduced using the minimum, nominal, and maximum estimated values of $C_{q \mathrm{Cal}}$. Because a single value of $C_{q C a l}$ applies to all data points within a given validation data set, error in $C_{q \mathrm{Cal}}$ results in a bias in the $\Delta C_{q}$ results but does not distort the shape of the original curve. Similar results can also be obtained for the other validation data sets. This means that although the exact values of $\Delta C_{q}$ presented here are uncertain, the differences in $\Delta C_{q}$ between two flow angles in the same data set are not. Thus, it is clear that the error in dynamic pressure varies considerably over the flow angles observed here, regardless of the value of this error. 


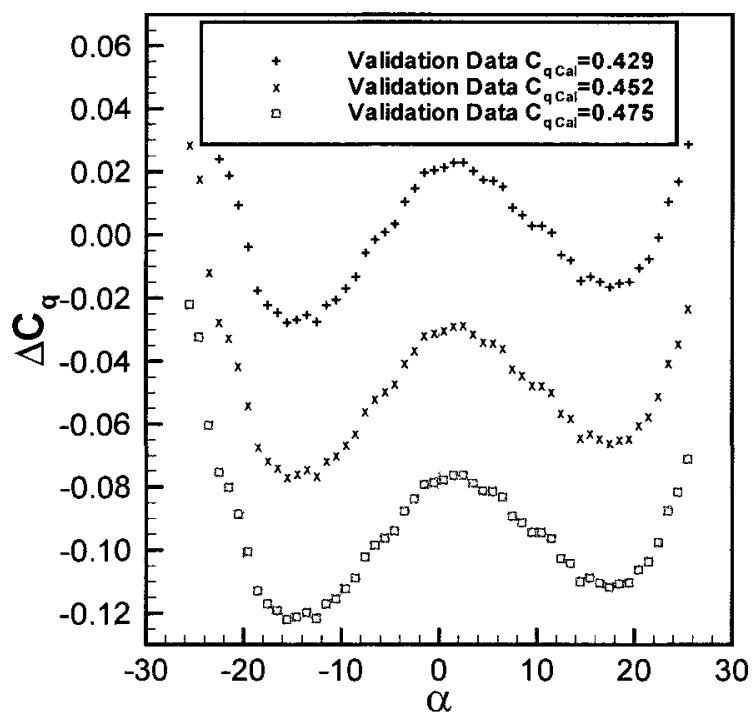

Figure 4.17: Effect of Uncertainty in $\mathrm{C}_{\mathrm{q} C a l}$ on Three-Hole Probe Dynamic Pressure Results using a Calibration at $\operatorname{Re}_{\mathrm{D}}=1850, T u=1.5 \%$ and Validation data at $\operatorname{Re}_{\mathrm{D}}=1850, T u=12 \%$

To compare the results for dynamic pressure with the previous results for total pressure, Figure 4.18 shows the off-calibration data from Figure 4.16 adjusted such that $\Delta C_{q}=0$ at $\alpha=0$. This allows for the RMS and maximum values of $\Delta C_{q}$ to be presented similar to those for total pressure shown in Figures 4.14 and 4.15. Figures 4.19 and 4.20 plot the RMS and maximum dynamic pressure errors, respectively, as a function of the resultant and streamwise turbulence intensities of the validation data; approximate curve fits are also included in each figure to highlight trends in the results. These figures show that, similar to results for total pressure, the magnitude of the error in dynamic pressure increases non-linearly with turbulence intensity. This non-linearity, however, is less pronounced than that for the error in total pressure due to turbulence intensity. 


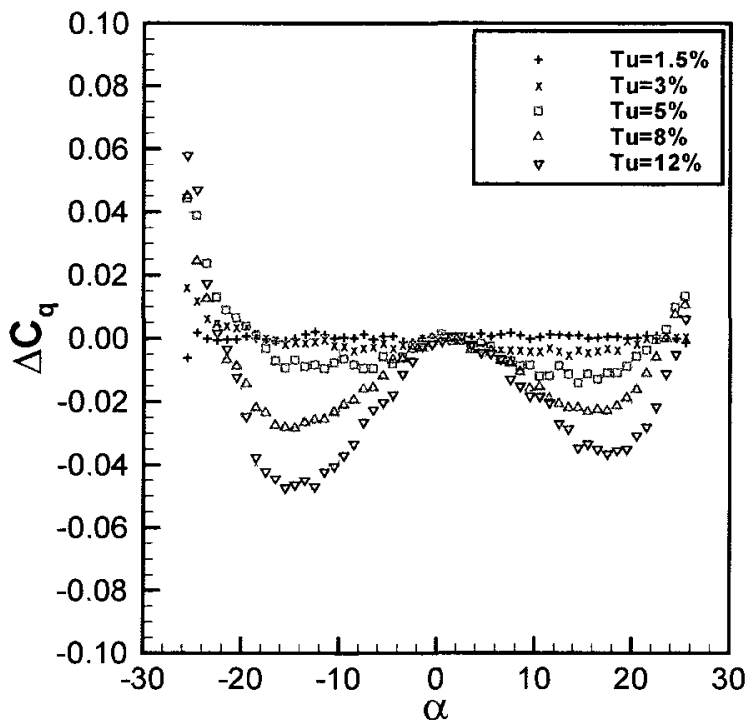

Figure 4.18: Three-Hole Pressure Probe Dynamic Pressure Error due to Turbulence Intensity using a Calibration at $\operatorname{Re}_{\mathrm{D}}=2500, \mathrm{Tu}=1.5 \%$, Modified to $\Delta \mathrm{C}_{\mathrm{q}}=0$ at $\alpha=0$

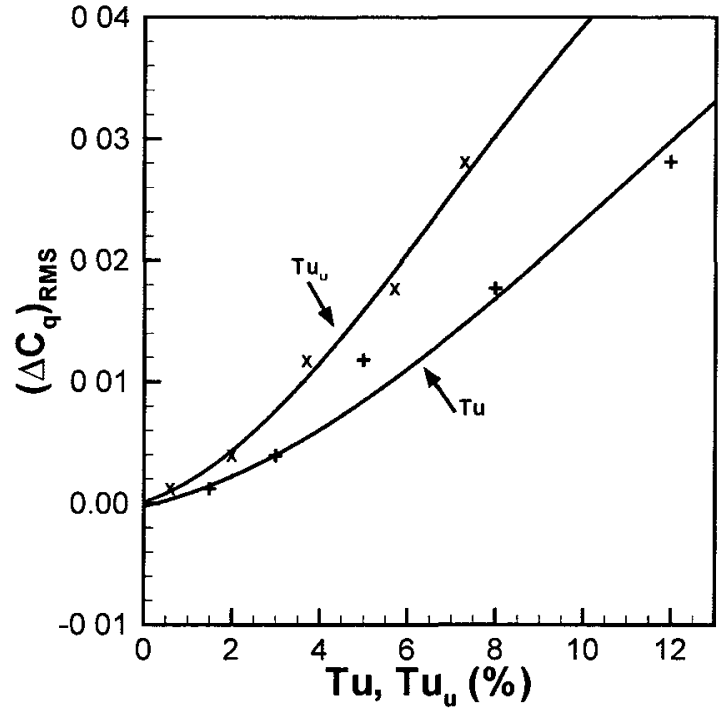

Figure 4.19: Three-Hole Pressure Probe RMS Dynamic Pressure Error due to Turbulence Intensity using a Calibration at $\operatorname{Re}_{D}=2500$ and $\mathrm{Tu}=1.5 \%$ 


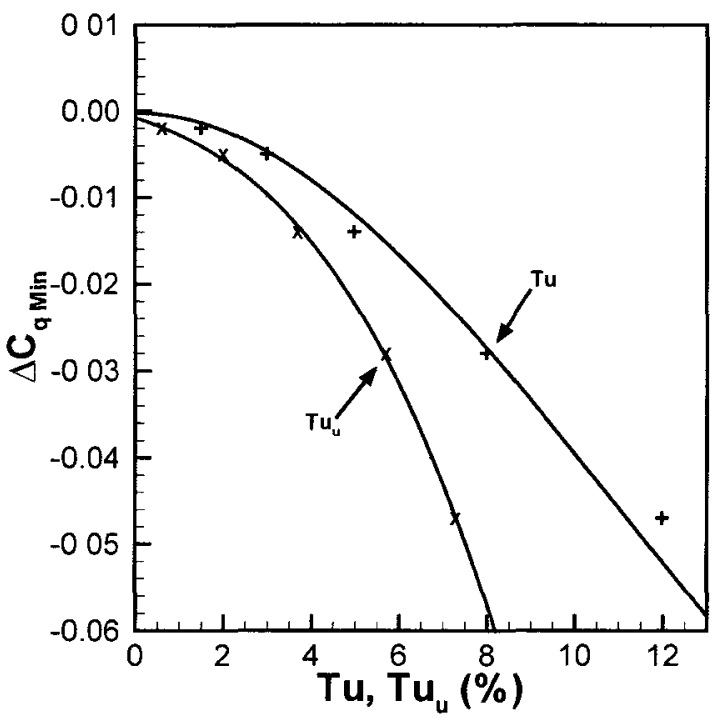

Figure 4.20: Three-Hole Pressure Probe Maximum Dynamic Pressure Error due to Turbulence Intensity using a Calibration at $R_{D}=2500$ and $T u=1.5 \%$

\subsubsection{Effects of Reynolds Number on Three-Hole Pressure Probe Flow Measurements at Off-Calibration Conditions}

The effects of reducing three-hole probe data at off-calibration Reynolds numbers are shown below by reducing data collected at three Reynolds numbers from 1850 to 4100 using a calibration at a Reynolds number of 4100 . All data sets presented here were collected at a resultant turbulence intensity of $1.5 \%$; results for other turbulence intensities are shown in Appendix C. The reader should note that the results shown below are not entirely novel: Section 2.3 .2 previously discussed the work of Benner (2003) who investigated the effects of Reynolds number on three-hole probes over yaw angles of $\pm 10^{\circ}$. Although the present study explores a wider range of angles $\left( \pm 25.5^{\circ}\right)$, comparison to Benner's results is important and also included below. 
Figure 4.21 shows the results for flow angle from the present study using a calibration taken $\operatorname{Re}_{D}=4100$ to reduce data also collected at that Reynolds number. Figures 4.22 and 4.23 show results using the same calibration to reduce validation data Reynolds numbers of 2500 and 1850 . For comparison, flow angle results from Benner are included below as Figure 4.24 .

Figure 4.24 shows that, at off-calibration conditions, flow angle results from Benner were centred about $\Delta \alpha \approx 0^{\circ}$ and displayed no systematic error due to Reynolds number. Results from the present study in Figures 4.22 and 4.23 however are centred about $\Delta \alpha \approx-0.4^{\circ}$ and show sinusoidal trends in $\Delta \alpha$. The translation of the data by $\Delta \alpha \approx-0.4^{\circ}$ is likely a result of probe misalignment during data collection, but the sinusoidal trend is not as easily explained.

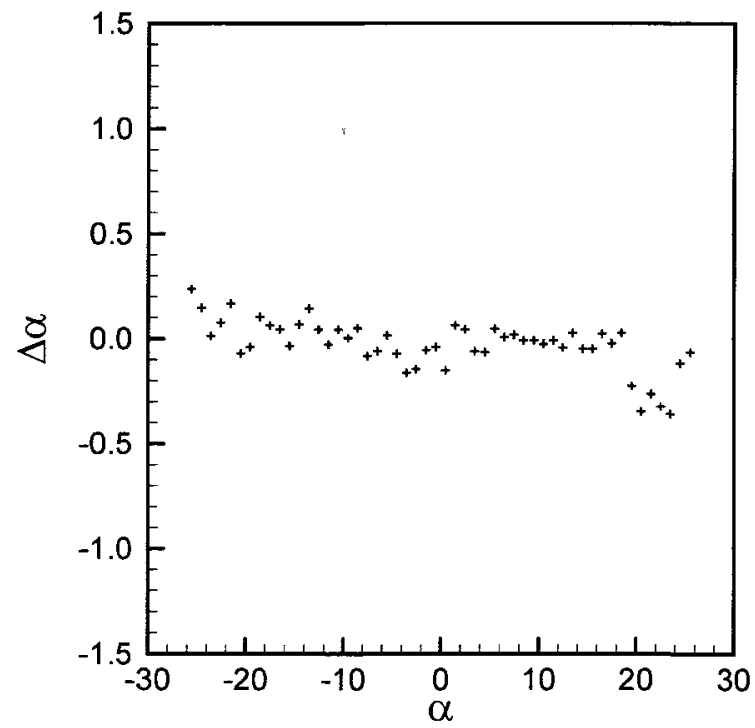

Figure 4.21: Three-Hole Pressure Probe Results for Flow Angle using Calibration and Validation Data at $\operatorname{Re}_{\mathrm{D}}=\mathbf{4 1 0 0 ,} \mathrm{Tu}=1.5 \%$ 


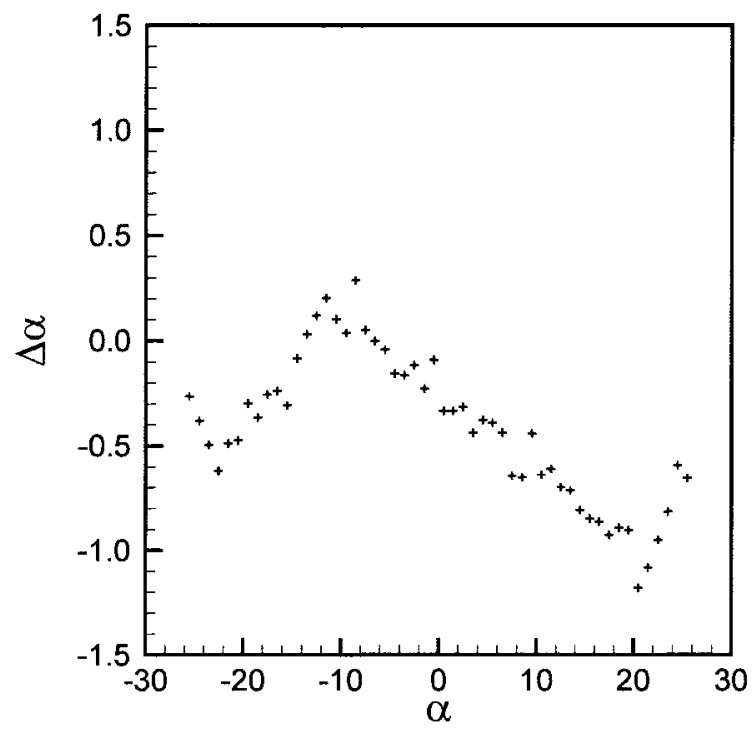

Figure 4.22: Three-Hole Pressure Probe Flow Angle Error due to Reynolds Number using a Calibration at $\operatorname{Re}_{\mathrm{D}}=4100, T u=1.5 \%$ and Validation Data $\operatorname{Re}_{\mathrm{D}}=2500, \mathrm{Tu}=1.5 \%$

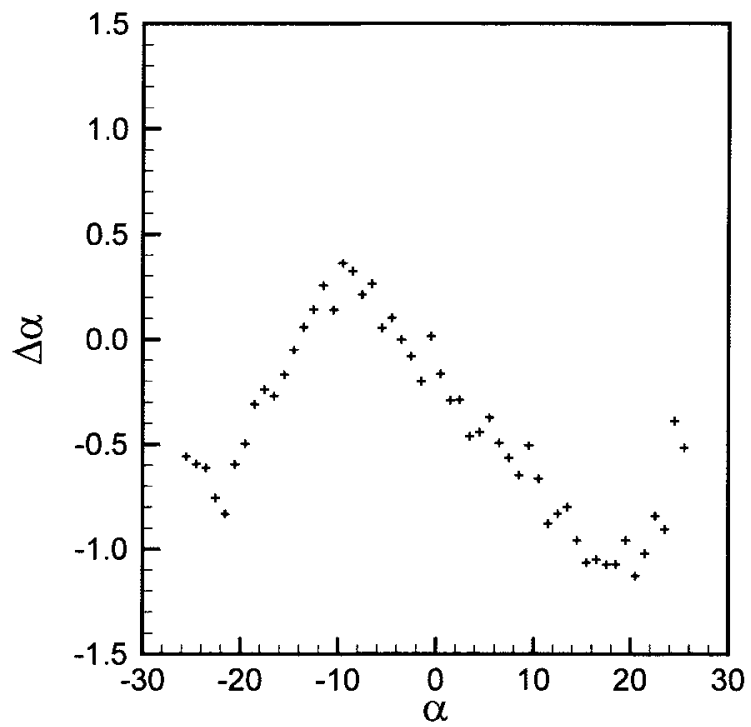

Figure 4.23: Three-Hole Pressure Probe Flow Angle Error due to Reynolds Number using a Calibration at $\operatorname{Re}_{\mathrm{D}}=\mathbf{4 1 0 0}, \mathrm{Tu}=1.5 \%$ and Validation Data at $\operatorname{Re}_{\mathrm{D}}=1850, \mathrm{Tu}=1.5 \%$ 


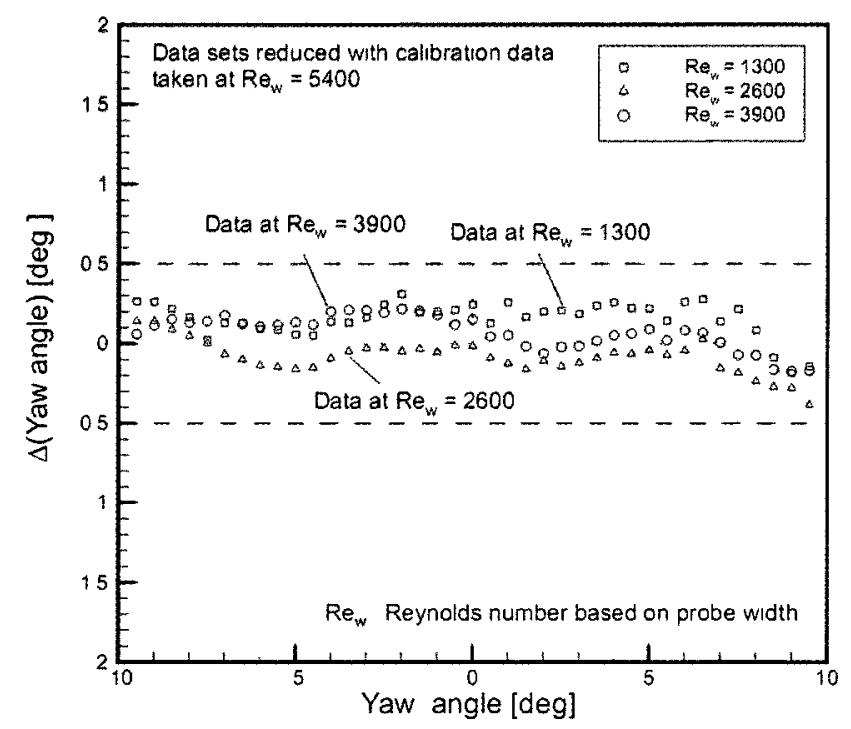

Figure 4.24: Three-Hole Pressure Probe Flow Angle Results for Various Reynolds Numbers, from Benner (2003)

Reynolds number is an indicator of the relative influence of the viscous and inertial forces in a flow. Large Reynolds numbers indicate flows with dominant inertial forces; small Reynolds numbers indicate flows with dominant viscous forces. The dependence of any flow phenomenon on Reynolds number typically indicates that the phenomenon is dependent on the relative effect of the viscosity in the flow. Thus, the dependence of the results for flow angle here on Reynolds number suggests that they are influenced by viscosity. In addition to this viscous influence, Section 4.4 .2 previously discussed the influence turbulence intensity on flow angle results reduced at off-calibration conditions and found similar sinusoidal trends. One example of these results, previously shown as Figure 4.12 , is repeated below as Figure 4.25 . Given that off-calibration turbulence intensities and Reynolds numbers both cause similar errors in the results for flow angle, it 
is suggestive that the mechanism by which the three-hole probe calibration is changing is related to transition or flow separation.

As discussed in Section 2.3.2, Johnson (1985) and Dominy and Hodson (1993) both investigated the effect of Reynolds number on five-hole pressure probes. Johnson found that, even at yaw angles as small as $8^{\circ}$, flow separation occurred on the leeward side of the tip of a five-hole probe. This separation originated at the leading edge of the probe and, depending on the flow angle and Reynolds number, could extend over part or all of the downstream yaw port. Building on these results, Dominy and Hodson found that this separation affected the yaw calibration coefficient of the probe and therefore the measurement of yaw angles. Dominy and Hodson thus concluded that Reynolds number influenced the results for flow angle from the probe by influencing this flow separation.

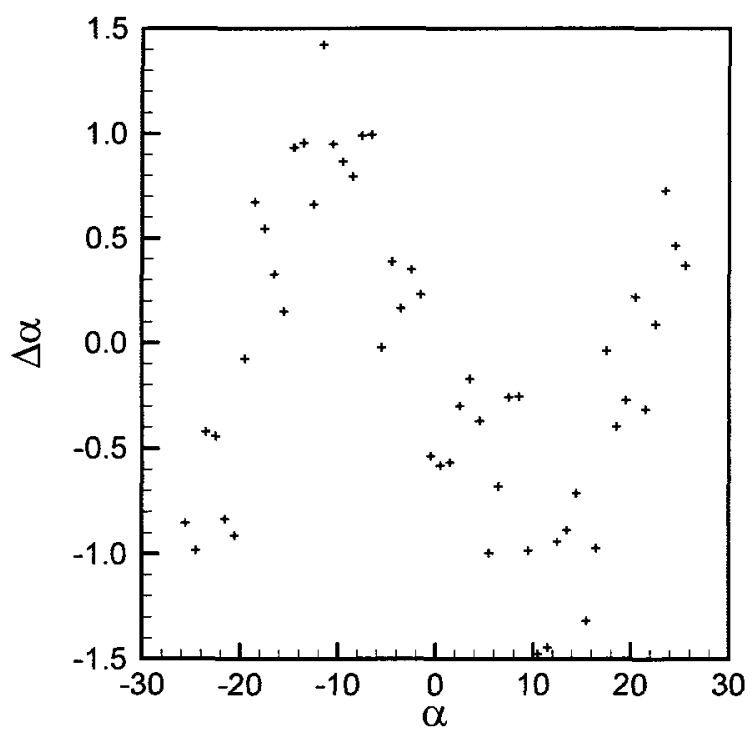

Figure 4.25: Three-Hole Pressure Probe Flow Angle Error due to Turbulence Intensity using a Calibration at $\operatorname{ReD}=2500, T u=1.5 \%$ and Validation Data at $\operatorname{ReD}=2500, T u=12 \%$ 
In addition to Reynolds number effects, Dominy and Hodson briefly investigated the influence turbulence intensity had on probe calibration. Because free-stream turbulence is known to influence the transition process, they proposed that it will also influence the nature of the flow separation on the probe and thus the yaw calibration. By comparing results obtained at turbulence intensities of less than $0.1 \%$ and approximately $10 \%$, Dominy and Hodson demonstrated that turbulence intensity did in fact influence the flow angle calibration of a five-hole probe. They did not however elaborate further on the magnitude of this effect or on its influence on the measurement of other flow properties using the probe.

Dominy and Hodson examined their five-hole probes at probe Reynolds numbers of 10000 and greater, but the present study examines probe behaviour at Reynolds numbers of less than about 5000. Given that smaller Reynolds numbers indicate flows in which viscosity has a greater influence, however, it is likely that the conclusions of Dominy and Hodson are also applicable to the present study. This suggests that the sinusoidal trends in the results for flow angle here may be related to a flow separation on the tip of the probe. To determine why the present results differ from those of Benner, we can compare the geometry of the probes used in both studies. Figure 4.26 shows the tip geometry of the two probes.

The most significant difference between the probes is their cone angles: the probe from the present study has a cone angle of $60^{\circ}$; the probe used by Benner has a cone angle of $90^{\circ}$. Typically, probes with smaller cone angles are more sensitive to changes in 


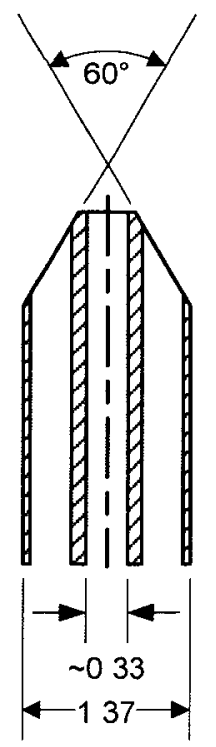

Dimensions in $\mathrm{mm}$

a) Probe from Present Study

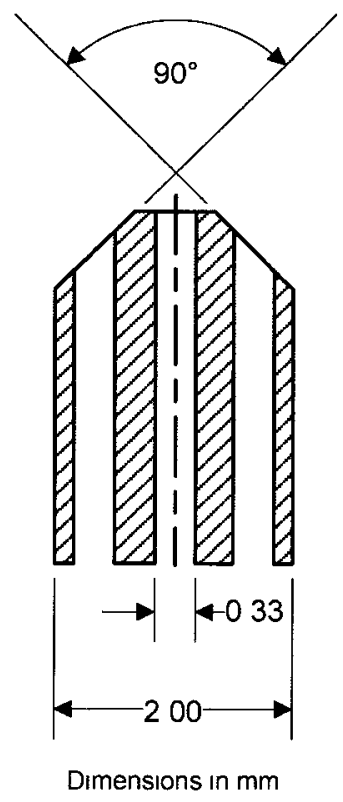

b) Probe from Benner (2003)

Figure 4.26: Comparison of the Three-Hole Probes from the Present Study and Benner (2003)

flow angle than are probes with larger cone angles. While comparing five-hole probes with different cone angles, Dominy and Hodson found that probes with a cone angle of $60^{\circ}$ were especially sensitive to the effects of Reynolds number on flow angle measurements but that probes with cone angles of $90^{\circ}$ were almost insensitive to these effects. They concluded that this difference was because flows were less likely to separate from the tips of probes with larger cone angles than from those with smaller cone angles. Thus, although the results for flow angle from Benner are in disagreement with those shown here, both are still consistent with previous research.

Figure 4.27 presents results for total pressure at off-calibration Reynolds numbers. Validation data collected at three Reynolds numbers was reduced using a calibration at a 
Reynolds number of 4100 . For comparison, similar measurements for total pressure from Benner are included as Figure 4.28.

The results for total pressure from Benner show that, for flow angles of less than $10^{\circ}$, his probe is insensitive to Reynolds number. These results are comparable to those collected by the present author at small flow angles. At large flow angles, however, the present study found that reducing validation data taken at a lower Reynolds number using a calibration at a higher Reynolds number resulted in a systematic underestimation of total pressure that increased with flow angle. This underestimation was as much as $2 \%$ of dynamic pressure for the operating points examined here. The response of the probe investigated here was also asymmetric (errors at positive flow angles were greater than errors at negative flow angles). This is likely due to some asymmetry in the probe tip.

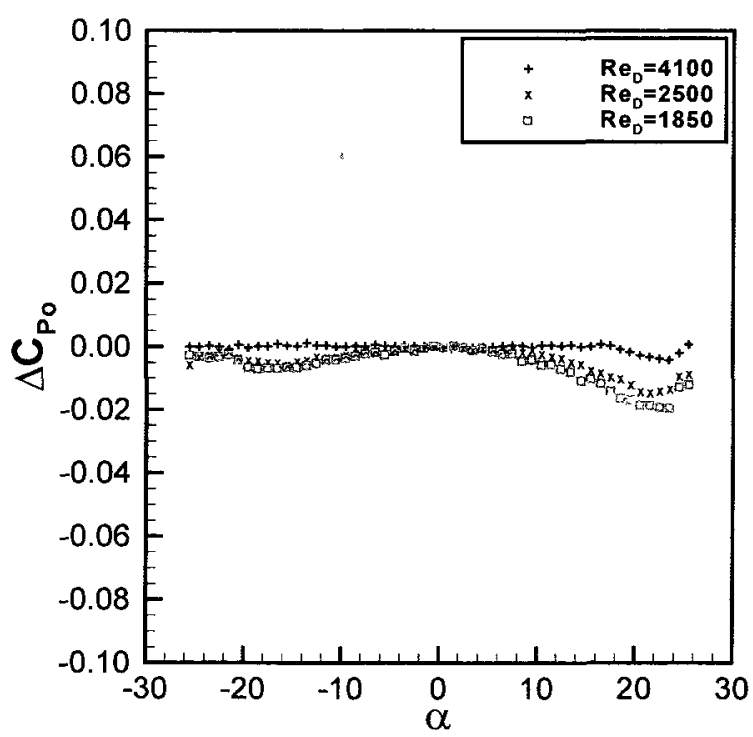

Figure 4.27: Three-Hole Pressure Probe Total Pressure Error due to Reynolds Number using a Calibration at $\operatorname{Re}_{\mathrm{D}}=\mathbf{4 1 0 0}, \mathrm{Tu}=\mathbf{1 . 5 \%}$ 


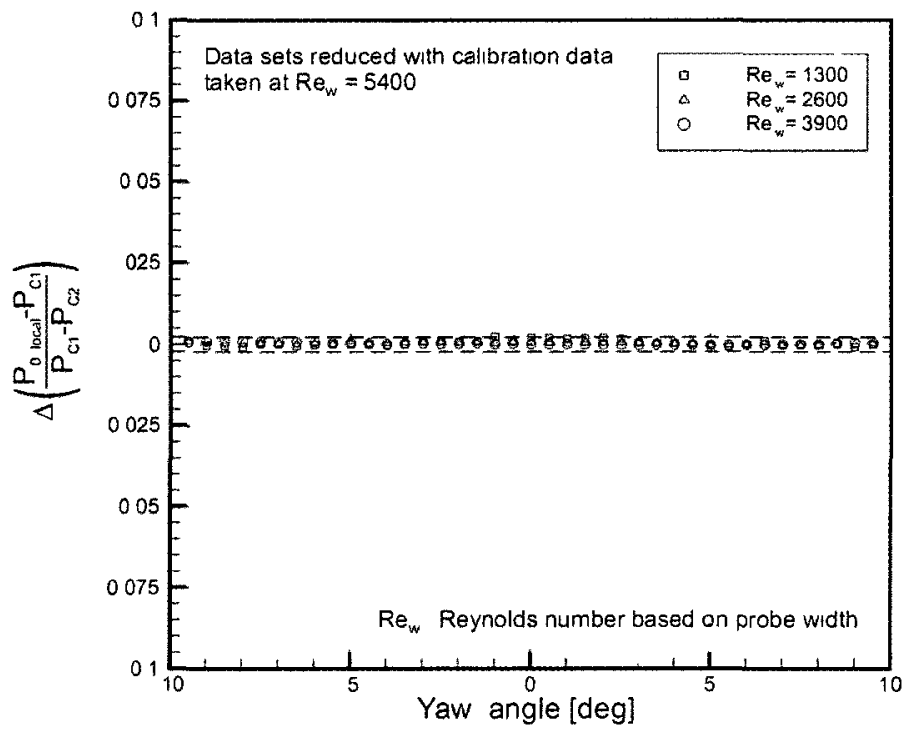

Figure 4.28: Three-Hole Pressure Probe Total Pressure Results for Various Reynolds Numbers, from Benner (2003)

Off-calibration results for dynamic pressure are shown in Figure 4.29; Figure 4.30 presents results collected by Benner at similar flow conditions. Results from the present study show that calibrating at $\operatorname{Re}_{D}=4100$ to reduce validation data at $R e_{D}=2500$ and $R e_{D}=1850$ results in a bias error in dynamic pressure of approximately $-4 \%$ and $-7 \%$, respectively, for $|\alpha|<20^{\circ}$. These trends are in reasonable agreement with those shown by Benner who reduced data collected at Reynolds numbers of 1300 to 3900 using a calibration collected at 5400 . Probe behaviour for $|\alpha|>20^{\circ}$ is erratic and may suggest some sort of flow separation on the probe tip, such as described previously for flow angle, although this is speculative. It is unclear why this erratic behaviour is only present in results for dynamic pressure. 


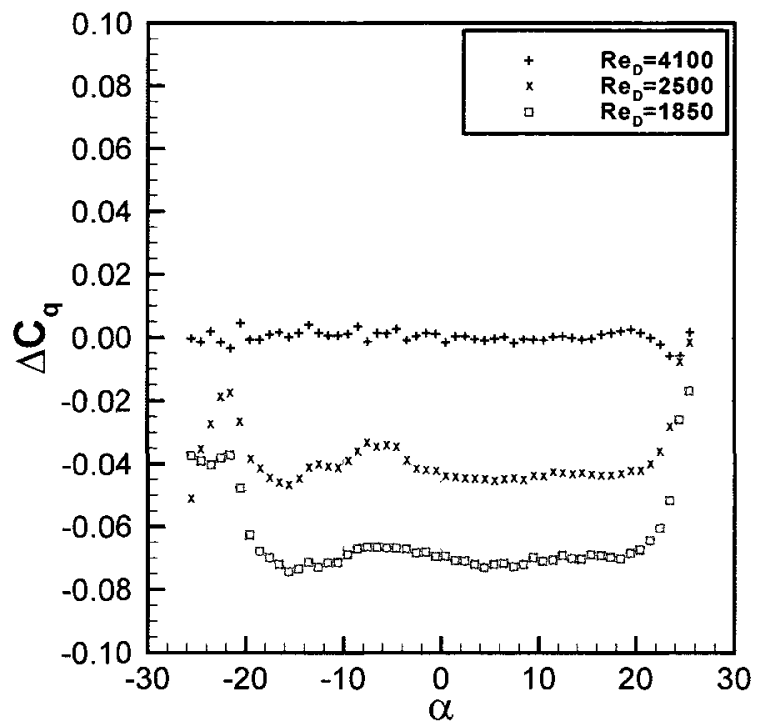

Figure 4.29: Three-Hole Pressure Probe Dynamic Pressure Error due to Reynolds Number using a Calibration at $\operatorname{Re}_{\mathrm{D}}=\mathbf{4 1 0 0}, \mathrm{Tu}=\mathbf{1 . 5 \%}$

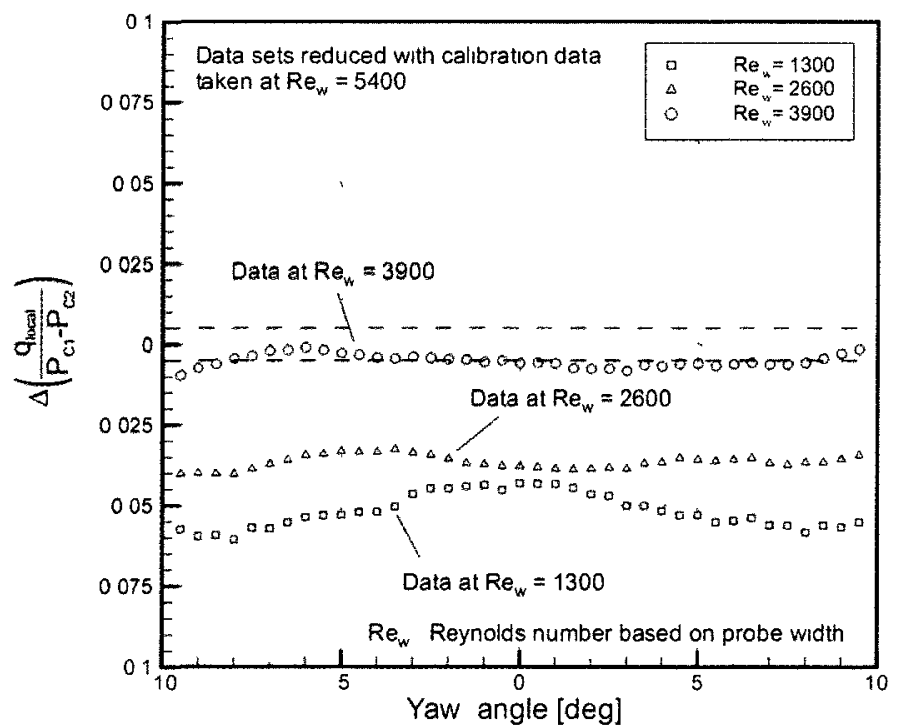

Figure 4.30: Three-Hole Pressure Probe Dynamic Pressure Results for Various Reynolds Numbers, from Benner (2003) 


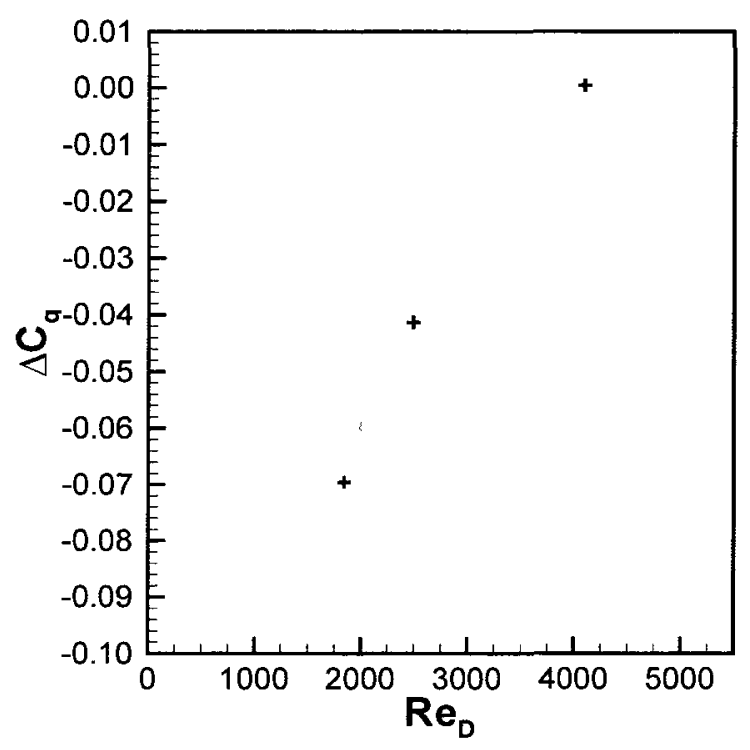

Figure 4.31: Three-Hole Pressure Probe Dynamic Pressure Error due to Reynolds Number using a Calibration at $\mathrm{Re}_{\mathrm{D}}=\mathbf{4 1 0 0}$ and $\mathrm{T} u=1.5 \%$

Figure 4.31 summarizes the results shown above for dynamic pressure by plotting them against the Reynolds numbers of the data. Given the limited number of data points, it is unclear whether the relation between the error and Reynolds number is linear or parabolic. It seems likely, however, that the influence of Reynolds number should diminish at higher Reynolds numbers as inertial forces become dominant in the flow.

\subsubsection{Summary}

The effects of turbulence intensity and Reynolds number on the three-hole pressure probe are summarized qualitatively in Tables 4.6 and 4.7. These tables include a summary of the results obtained using the high-turbulence and low-Reynolds number calibrations in Appendix $\mathrm{C}$ and the precision errors as discussed in Section 3.4.3. 


\begin{tabular}{|c|c|c|c|}
\hline & \multicolumn{2}{|c|}{ Validation Data at: } \\
\hline & & Low Turbulence & High Turbulence \\
\hline 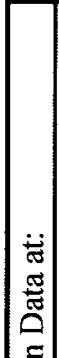 & 总 & $\begin{array}{l}\text { Precision Error: minimal } \\
\alpha: \text { no systematic error } \\
P_{o}: \text { no systematic error } \\
q: \text { no systematic error }\end{array}$ & $\begin{array}{l}\text { Precision Error: significant } \\
\alpha: \text { complex, sinusoidal systematic error related } \\
\text { to flow separation on the probe. See Section } \\
4.4 .2 \\
\text { Po: minimal error at } \alpha=0^{\circ} \text {, significant } \\
\text { underestimation that increases with } \alpha \text { for } \alpha>0^{\circ} \\
q: \text { complex response including significant } \\
\text { error. See Section } 4.4 .2\end{array}$ \\
\hline | & 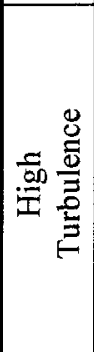 & $\begin{array}{l}\text { Precision Error: significant } \\
a: \text { complex, sinusoidal systematic error related } \\
\text { to flow separation on the probe. See Section } \\
4.4 .2 \\
P_{o}: \text { minimal error at } \alpha=0^{\circ} \text {, significant } \\
\text { overestimation that increases with } \alpha \text { for } \alpha>0^{\circ} \\
q: \text { complex response including significant } \\
\text { error. See Section } 4.4 .2\end{array}$ & $\begin{array}{l}\text { Precision Error: significant } \\
a: \text { no systematic error } \\
\boldsymbol{P}_{o}: \text { no systematic error } \\
q: \text { no systematic error }\end{array}$ \\
\hline
\end{tabular}

Table 4.6: Summary of the Effects of Turbulence on Three-Hole Pressure Probe

Measurements

\begin{tabular}{|c|c|c|c|}
\hline & \multicolumn{2}{|c|}{ Validation Data at: } \\
\hline & & Low Reynolds Number & High Reynolds Number \\
\hline 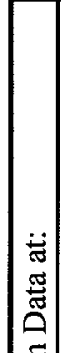 & 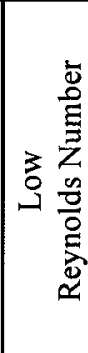 & $\begin{array}{l}\text { Precision Error: minimal } \\
\alpha: \text { no systematic error } \\
P_{o}: \text { no systematic error } \\
q: \text { no systematic error }\end{array}$ & $\begin{array}{l}\text { Precision Error: minimal } \\
\alpha: \text { complex, sinusoidal systematic error related } \\
\text { to flow separation on the probe. See Section } \\
4.4 .3 \\
\boldsymbol{P}_{o}: \text { no error at } \alpha=0^{\circ} \text {, very small } \\
\text { overestimation that increases with } \alpha \text { for } \alpha \gg 0^{\circ} \\
\text { and } R e_{C a l} \ll R e_{V a l} \\
q: \text { significant overestimation bias at all } \alpha\end{array}$ \\
\hline 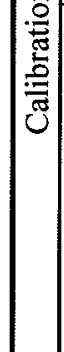 & 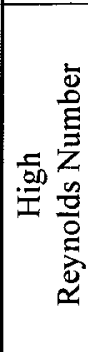 & $\begin{array}{l}\text { Precision Error: minimal } \\
\alpha: \text { complex, sinusoidal systematic error related } \\
\text { to flow separation on the probe. See Section } \\
4.4 .3 \\
P_{o}: \text { no error at } \alpha=0^{\circ} \text {, very small } \\
\text { underestimation that increases with } \alpha \text { for } \\
\alpha \gg 0^{\circ} \text { and } R e_{C a l} \gg R e_{V a l} \\
q: \text { significant underestimation bias at all } \alpha\end{array}$ & $\begin{array}{l}\text { Precision Error: minimal } \\
\alpha: \text { no systematic error } \\
\boldsymbol{P}_{o}: \text { no systematic error } \\
q: \text { no systematic error }\end{array}$ \\
\hline
\end{tabular}

Table 4.7: Summary of the Effects of Reynolds Number on Three-Hole Pressure Probe Measurements 


\subsection{Effects of Turbulence Intensity and Reynolds Number on Seven-Hole Pressure Probes}

\subsubsection{Introduction}

To the knowledge of the present author, the effects of turbulence intensity on the aerodynamic behaviour of the seven-hole pressure probe at off-calibration conditions have not been fully described in the open literature. Past investigations, such as those by Dominy and Hodson (1993) and Takahashi (1997), qualitatively describe some of the effects of turbulence intensity on five- and seven-hole probes, but none provide full quantitative details. The following section estimates these effects using the same methodology as applied in the previous section for the three-hole probe. In addition to the turbulence effects, the effects of Reynolds numbers are also investigated. It should be noted, however, that previous studies on the effects of Reynolds numbers on seven-hole probe behaviour include Wenger and Devenport (1999) and MacIsaac et al. (2009).

Calibration and validation data sets were collected at five resultant turbulence intensities varying from 1.5 to $12 \%$ and Reynolds numbers ranging from 2000 to 5150 . Each calibration data set consisted of measurements collected in a known flow over a grid of pitch and yaw misalignment angles ranging from -30 to $30^{\circ}$ in $2^{\circ}$ steps. Validation data were collected in a similar manner over angles of -25.5 to $25.5^{\circ}$ in $3^{\circ}$ steps. Tables 4.8 and 4.9 list the calibration and validation data sets used to investigate 
the effects of turbulence intensity on the probe; Tables 4.10 and 4.11 list the data sets used for Reynolds number investigations.

\begin{tabular}{|c|c|cc|c|c|c|c|}
\hline $\begin{array}{c}\mathrm{Re}_{\mathrm{D}} \\
\text { [nominal] }\end{array}$ & \multicolumn{2}{|c|}{$\begin{array}{c}T u(\%) \\
{[\text { nominal] }}\end{array}$} & $\begin{array}{c}T u_{u} \\
(\%)\end{array}$ & $\begin{array}{c}T u_{v} \\
(\%)\end{array}$ & $\begin{array}{c}T u_{w} \\
(\%)\end{array}$ & $\begin{array}{c}\Lambda_{x} \\
(\mathrm{~mm})\end{array}$ \\
\hline 2500 & {$[2500]$} & 1.7 & {$[1.5]$} & 0.6 & 2.5 & 1.3 & - \\
\hline 3920 & {$[3900]$} & 1.7 & {$[1.5]$} & 0.6 & 2.4 & 1.7 & - \\
\hline 2490 & {$[2500]$} & 3.1 & {$[3]$} & 2.0 & 3.9 & 3.1 & 7.3 \\
\hline 3910 & {$[3900]$} & 2.9 & {$[3]$} & 2.0 & 3.6 & 2.9 & 5.6 \\
\hline 2490 & {$[2500]$} & 5.3 & {$[5]$} & 4.0 & 5.6 & 6.0 & 4.6 \\
\hline 3930 & {$[3900]$} & 5.0 & {$[5]$} & 3.7 & 5.4 & 5.6 & 4.0 \\
\hline 2470 & {$[2500]$} & 7.7 & {$[8]$} & 5.7 & 8.4 & 8.6 & 5.4 \\
\hline 3910 & {$[3900]$} & 8.1 & {$[8]$} & 5.7 & 9.0 & 9.2 & 5.3 \\
\hline 2470 & {$[2500]$} & 12.2 & {$[12]$} & 7.3 & 14.2 & 13.7 & 7.4 \\
\hline 3890 & {$[3900]$} & 12.4 & {$[12]$} & 7.3 & 14.7 & 14 & 7.5 \\
\hline
\end{tabular}

Table 4.8: Seven-Hole Pressure Probe Calibration Test Matrix for Investigating the Influence of Turbulence Intensity

\begin{tabular}{|c|c|cc|c|c|c|c|}
\hline $\begin{array}{c}\mathrm{Re}_{\mathrm{D}} \\
\text { [nominal] }\end{array}$ & $\begin{array}{c}|c| \\
\text { [nominal] }\end{array}$ & $\begin{array}{c}T u_{u} \\
(\%)\end{array}$ & $\begin{array}{c}T u_{v} \\
(\%)\end{array}$ & $\begin{array}{c}T u_{w} \\
(\%)\end{array}$ & $\begin{array}{c}\Lambda_{x} \\
(\mathrm{~mm})\end{array}$ \\
\hline 2500 & {$[2500]$} & 1.7 & {$[1.5]$} & 0.6 & 2.5 & 1.3 & - \\
\hline 3930 & {$[3900]$} & 1.7 & {$[1.5]$} & 0.6 & 2.4 & 1.7 & - \\
\hline 2480 & {$[2500]$} & 3.1 & {$[3]$} & 2.0 & 3.9 & 3.1 & 7.3 \\
\hline 3890 & {$[3900]$} & 2.9 & {$[3]$} & 2.0 & 3.6 & 2.9 & 5.6 \\
\hline 2500 & {$[2500]$} & 5.3 & {$[5]$} & 4.0 & 5.6 & 6.0 & 4.6 \\
\hline 3930 & {$[3900]$} & 5.0 & {$[5]$} & 3.7 & 5.4 & 5.6 & 4.0 \\
\hline 2480 & {$[2500]$} & 7.7 & {$[8]$} & 5.7 & 8.4 & 8.6 & 5.4 \\
\hline 3930 & {$[3900]$} & 8.1 & {$[8]$} & 5.7 & 9.0 & 9.2 & 5.3 \\
\hline 2470 & {$[2500]$} & 12.2 & {$[12]$} & 7.3 & 14.2 & 13.7 & 7.4 \\
\hline 3870 & {$[3900]$} & 12.4 & {$[12]$} & 7.3 & 14.7 & 14 & 7.5 \\
\hline
\end{tabular}

Table 4.9: Seven-Hole Pressure Probe Validation Test Matrix for Investigating the Influence of Turbulence Intensity 


\begin{tabular}{|c|c|c|c|c|c|}
\hline $\begin{array}{c}\operatorname{Re}_{\mathrm{D}} \\
\text { [nominal] }\end{array}$ & $\begin{array}{c}T u(\%) \\
\text { [nominal] }\end{array}$ & $\begin{array}{l}T u_{u} \\
(\%)\end{array}$ & $\begin{array}{l}T u_{v} \\
(\%)\end{array}$ & $\begin{array}{l}T u_{w} \\
(\%)\end{array}$ & $\begin{array}{c}\Lambda_{x} \\
(\mathrm{~mm})\end{array}$ \\
\hline $1980 \quad[2000]$ & $1.4 \quad[1.5]$ & 0.6 & 1.8 & 1.1 & - \\
\hline $2490 \quad[2500]$ & $1.7 \quad[1.5]$ & 0.6 & 2.5 & 1.3 & - \\
\hline $2890 \quad[2900]$ & $1.7 \quad[1.5]$ & 0.6 & 2.5 & 1.5 & - \\
\hline$[3400]$ & $1.7 \quad[1.5]$ & 0.6 & 2.4 & 1.7 & - \\
\hline $4020 \quad[4000]$ & $1.8 \quad[1.5]$ & 0.6 & 2.2 & 2.1 & - \\
\hline $4390 \quad[4400]$ & [1.5] & 0.6 & 2.2 & 2.1 & - \\
\hline $5140 \quad[5150]$ & $1.7 \quad[1.5]$ & 0.7 & 2.4 & 1.6 & - \\
\hline$[2000]$ & $11.8 \quad[12]$ & 7.3 & 13.6 & 13.4 & 7.7 \\
\hline [2450] & $12.2[12]$ & 7.3 & 14.2 & 13.7 & 7.4 \\
\hline [2850] & 12.3 & 7.4 & 14.4 & 14.0 & 7.6 \\
\hline [3400] & [12] & 7.3 & 14.6 & 14 & 7.5 \\
\hline $3810 \quad[3800]$ & 12.4 & 7.3 & 14.7 & 14 & 7.5 \\
\hline
\end{tabular}

Table 4.10: Seven-Hole Pressure Probe Calibration Test Matrix for Investigating the Influence of Reynolds Number

\begin{tabular}{|c|c|c|c|c|c|c|c|}
\hline $\begin{array}{c}\mathrm{Re}_{\mathrm{D}} \\
\text { [nominal] }\end{array}$ & \multicolumn{2}{|c|}{$\begin{array}{c}T u(\%) \\
{[\text { nominal] }}\end{array}$} & $\begin{array}{c}T u_{u} \\
(\%)\end{array}$ & $\begin{array}{c}T u_{v} \\
(\%)\end{array}$ & $\begin{array}{c}T u_{w} \\
(\%)\end{array}$ & $\begin{array}{c}\Lambda_{x} \\
(\mathrm{~mm})\end{array}$ \\
\hline 2480 & {$[2500]$} & 1.7 & {$[1.5]$} & 0.6 & 2.5 & 1.3 & - \\
\hline 3410 & {$[3400]$} & 1.7 & {$[1.5]$} & 0.6 & 2.4 & 1.7 & - \\
\hline 4010 & {$[4000]$} & 1.8 & {$[1.5]$} & 0.6 & 2.2 & 2.1 & - \\
\hline 1960 & {$[1950]$} & 11.8 & {$[12]$} & 7.3 & 13.6 & 13.4 & 7.7 \\
\hline 2440 & {$[2450]$} & 12.2 & {$[12]$} & 7.3 & 14.2 & 13.7 & 7.4 \\
\hline 3340 & {$[3350]$} & 12.4 & {$[12]$} & 7.3 & 14.6 & 14 & 7.5 \\
\hline 3780 & {$[3800]$} & 12.4 & {$[12]$} & 7.3 & 14.7 & 14 & 7.5 \\
\hline
\end{tabular}

Table 4.11: Seven-Hole Pressure Probe Validation Test Matrix for Investigating the Influence of Reynolds Number

Similar to the flows investigated in the Kiel and three-hole probes studies, there is a notable anisotropy in the turbulence intensity of all operating points listed above. The length scale of the turbulence was also found to vary between operating points. As discussed previously in Sections 4.2 and 4.3 , the effects of these phenomena on the results presented here are expected to be small because, at all operating points, the 
anisotropy is similar and the dominant length scales are larger than the probe diameter $\left(\Lambda_{x} / \mathrm{D} \approx 2.9\right.$ to 5.5 ). It should still be noted, however, that most discussion is presented using the resultant turbulence intensity $(T u)$. Some results are also shown using the streamwise component of turbulence intensity $\left(T u_{u}\right)$.

Given their small dimensions, seven-hole probes are sensitive to minor surface damage, such as scratches, or the collection of debris on their tips that can change their aerodynamic behaviour and thus their calibrations. Scratches and debris by themselves are not problematic because the calibration procedure accounts for the condition of the probe at the time of calibration. If fouling occurs after the probe has been calibrated, however, it can cause errors in the results inferred from the probe. Therefore, it is critical to regularly check the calibrations of seven-hole probes to ensure fouling has not occurred. In the event that the probe becomes fouled or damaged, the probe must be cleaned and possibly recalibrated if cleaning does not return it to the original aerodynamic behaviour.

At one point during the present study it was identified that the seven-hole probe used here had become fouled. The probe was immediately cleaned and recalibrated, but the cleaned probe did not return to the same aerodynamic behaviour as the pre-fouled probe. This could be due to some debris that was on the probe prior to the original calibration or the result of minor damage. This fouling occurred after data collection for the Reynolds number testing was complete (Tables 4.10 and 4.11) and before data collection for the turbulence intensity had begun (Tables 4.8 and 4.9 ). Thus, the probe fouling does not 
affect the results presented here for either case but it does prevent the use of calibrations from the Reynolds number test group to reduce validation data from the turbulence intensity test group and vice versa. The fouling also emphasizes the need to periodically test the calibration of seven-hole probes to detect when fouling or damage occurs.

The results in this section compare the values of flow angle, total pressure, and dynamic pressure inferred from the seven-hole probe measurements to the true values. These comparisons are presented using both a tangential (Figure 4.32) and polar coordinate system (Figure 4.33) through the equations:

$$
\begin{gathered}
\Delta \theta=\theta_{\text {Inferred }}-\theta_{\text {True }} \\
\Delta C_{P_{o}}=\frac{P_{\text {oInferred }}-P_{\text {oTrue }}}{q_{\text {True }}} \\
\Delta C_{q}=\frac{q_{\text {Inferred }}-q_{\text {True }}}{q_{\text {True }}}
\end{gathered}
$$

where $\Delta C_{P o}$ and $\Delta C_{q}$ are the errors in total and dynamic pressure normalized by true dynamic pressure and $\Delta \theta$ is the angle in the resultant flow angle in degrees. All results were first calculated in the tangential coordinate system using the data reduction procedures described in Section 3.4.4 and then converted to the polar coordinate system. The polar coordinate system was better suited to identify and present the trends in the results because probe response was known to be approximately axisymmetric about $\theta=0^{\circ}$. Most of the results in this section are presented using the polar coordinate 
system; some colourflood contour plots in the tangential coordinate system are also included to demonstrate the axisymmetric nature of the results. It should be noted, however, that the author is not advocating that the data reduction be conducted in the polar coordinate system but simply using it to conveniently present the data.

Along with the results shown here for the seven-hole probe $7 \mathrm{HP1}$, some data were also collected using the larger probe 7HP2. This second probe was examined to determine if diameter influenced the effects of Reynolds number or turbulence intensity on probe aerodynamic behaviour. The present study found that results for $7 \mathrm{HP} 2(2.4 \mathrm{~mm}$ in diameter) were similar to those of 7HP1 (1.82 $\mathrm{mm}$ in diameter). Thus, results for 7HP2 have been omitted from the main text for brevity but are included in Appendix D.

\subsubsection{Effects of Turbulence Intensity on Seven-Hole Pressure Probe Flow Measurements at Off-Calibration Conditions}

The effect of reducing data at off-calibration turbulence intensities is demonstrated using a calibration collected at a resultant turbulence intensity of $1.5 \%$ to reduce validation data sets at 1.5 to $12 \%$. The data presented here were collected at a Reynolds number of 3900 ; results for a Reynolds number of 2500 were similar and are omitted. Given the large number of data points collected for each validation data set, all plots are presented using just trend lines for clarity. Plots with the full scatter for each case are available in Appendix D. 


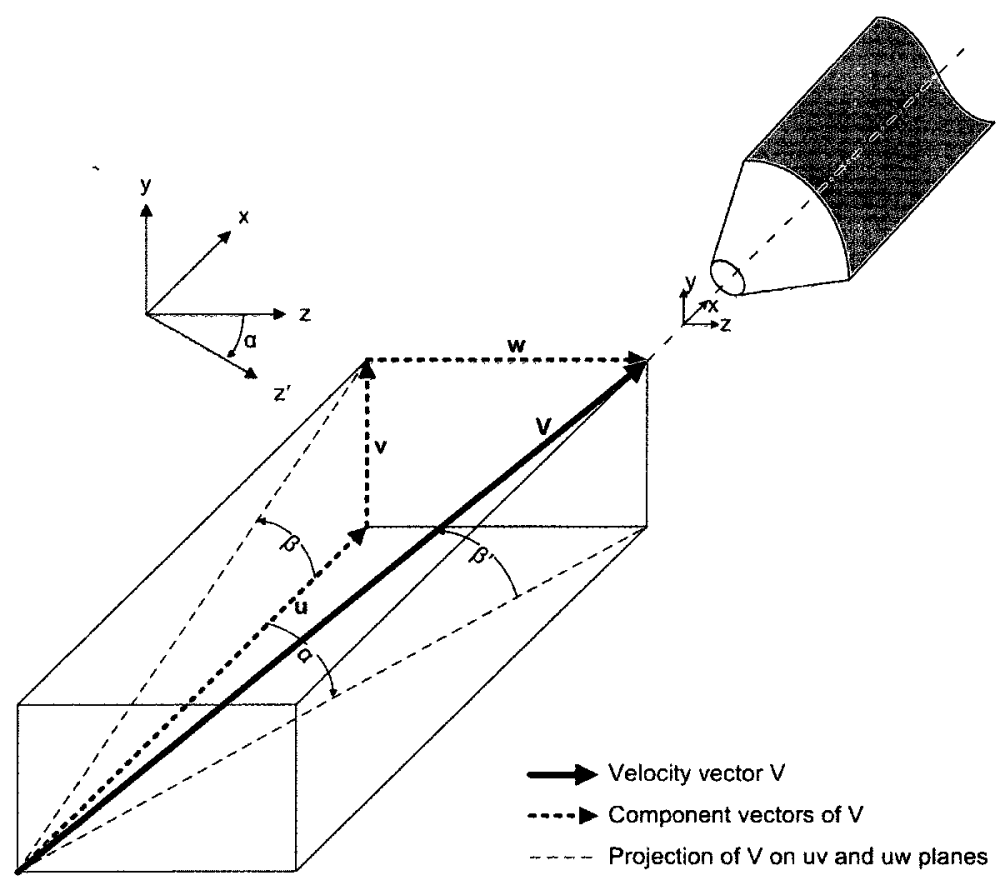

Figure 4.32: Probe Tangential Coordinate System, adapted from Gerner et al. (1984)

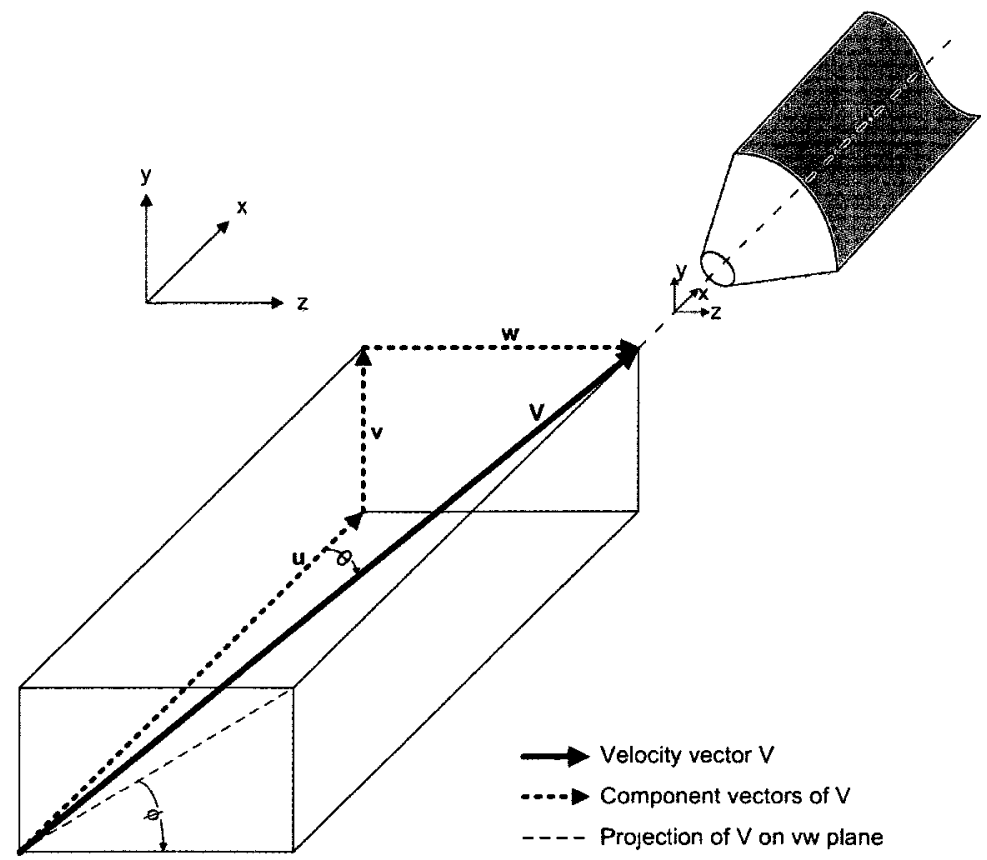

Figure 4.33: Probe Polar Coordinate System, adapted from Gerner et al. (1984) 
The off-calibration results for flow angle at all five turbulence intensities are shown in Figure 4.34. These results show that, for data collected at turbulence intensities of $8 \%$ or less, turbulence intensity has a relatively small effect on data reduction. Results from the $12 \%$ validation data show some underestimation of flow angle for $\theta<15^{\circ}$, but given that the scatter in those results was as much as $\pm 1.0^{\circ}$ (as shown in the full scatter plots included in Appendix D) it is difficult to determine if the trend shown here is the actual probe response or experimental uncertainty. Thus, in general, flow angle appears essentially unaffected by off-calibration turbulence intensities.

Figure 4.35 shows results for total pressure using the same validation data sets as discussed above. Additionally, Figure 4.36 presents detailed results for the $12 \%$ validation data using a colourflood contour plot of $\Delta C_{P o}$ as a function of $\alpha$ and $\beta$.

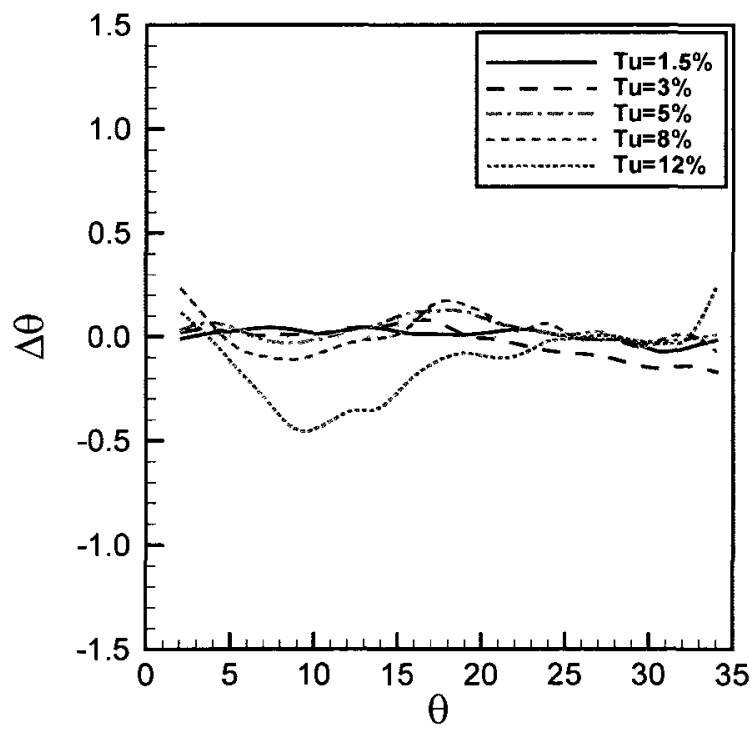

Figure 4.34: Seven-Hole Pressure Probe Flow Angle Error due to Turbulence Intensity using a Calibration at $\operatorname{Re}_{\mathrm{D}}=3900, \mathrm{Tu}=1.5 \%$ 
Colourflood plots for the other turbulence intensities are included in Appendix D and demonstrate similar trends. These results for total pressure show that reducing data at high turbulence intensities using a low-turbulence calibration results in an underestimation of total pressure. This systematic error is axisymmetric about $\alpha=0$, $\beta=0$ and minimal near $\theta=0$.

Figure 4.37 plots the maximum error for total pressure (in this case, the minimum values of $\Delta C_{P o}$ ) as a function of the resultant and streamwise turbulence intensities of the flow. This figure demonstrates that the effect increases non-linearly with turbulence intensity. The trend seen here for the seven-hole probe is similar to that discussed previously for the three-hole probe. The maximum errors in the seven-hole probe results, however, are greater than those in the three-hole probe results at comparable turbulence intensities.

$\Delta C_{P o}$ approaches 0 at $\theta=0^{\circ}$ for all operating points shown in Figure 4.35. As discussed in Section 4.4.2 for the three-hole probe, however, this result is a product of the method by which the true total pressure is determined and may not be the actual probe response. The values of true total pressure $\left(P_{o \text { True }}\right)$ at each operating point may include a bias error due to the effects of turbulence. This would in turn cause a bias in the results for $\Delta C_{P o}$ presented above. An estimate of the bias in $\Delta C_{P o}$ for each operating point is shown in Table 4.12; a full description of the cause of this bias and the methodology used to its estimate is included in Section 4.4.2. 


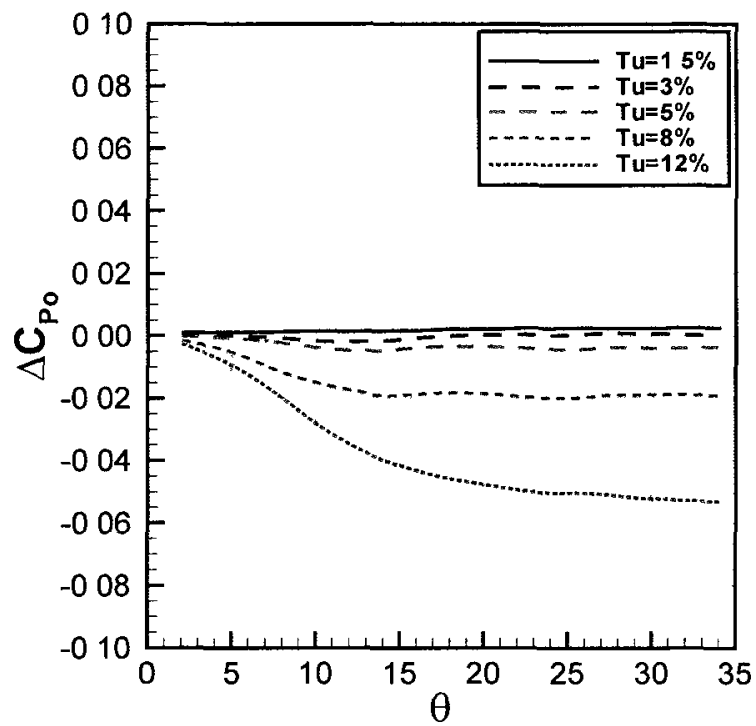

Figure 4.35: Seven-Hole Pressure Probe Total Pressure Error due to Turbulence Intensity using a Calibration at $\mathrm{Re}_{\mathrm{D}}=3900, \mathrm{Tu}=1.5 \%$

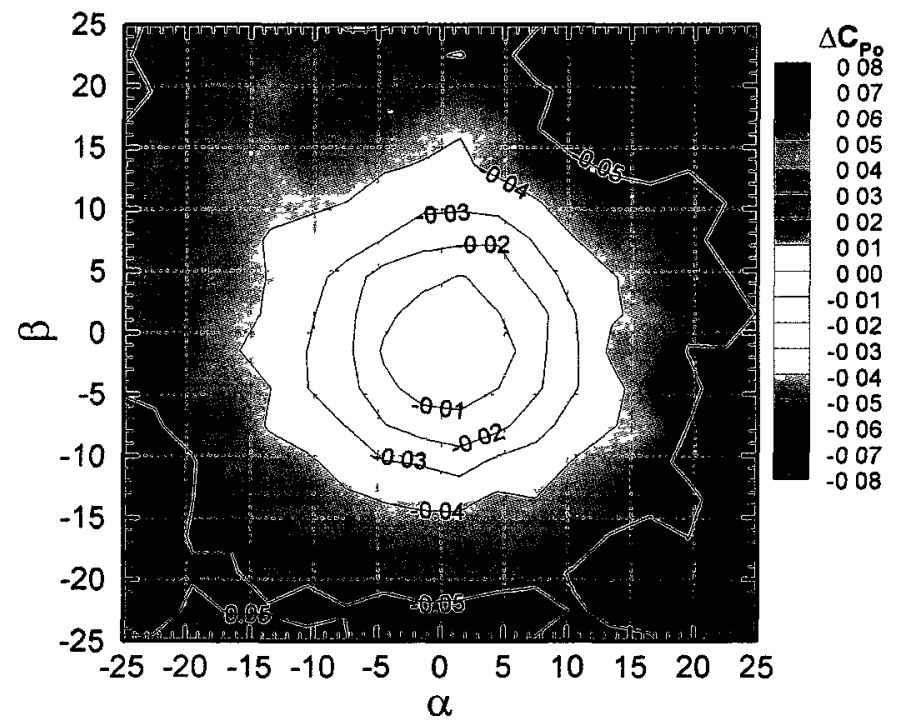

Figure 4.36: Seven-Hole Pressure Probe Total Pressure Error due to Turbulence Intensity using a Calibration at $R_{D}=3900, T u=1.5 \%$ and Validation Data at $\operatorname{Re}_{D}=3900, T u=12 \%$ 


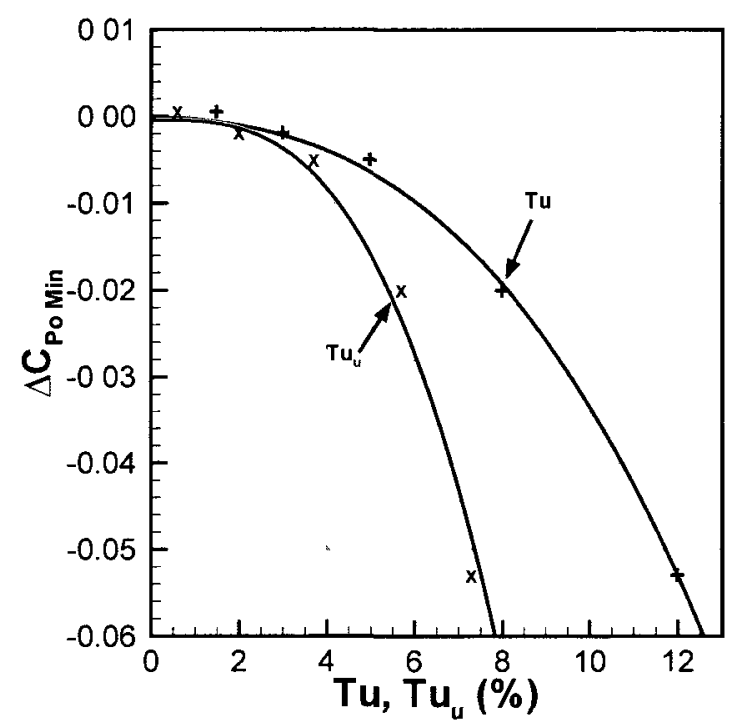

Figure 4.37: Seven-Hole Pressure Probe Maximum Total Pressure Error due to Turbulence Intensity using a Calibration at $\operatorname{Re}_{\mathrm{D}}=3900$ and $\mathrm{Tu}=\mathbf{1 . 5 \%}$

\begin{tabular}{|c|c|}
\hline$T u(\%)$ & $\Delta \mathrm{C}_{\text {PoBas }}$ \\
\hline 1.5 & -0.0002 \\
\hline 3 & -0.0009 \\
\hline 5 & -0.0025 \\
\hline 8 & -0.0064 \\
\hline 12 & -0.014 \\
\hline
\end{tabular}

Table 4.12: Estimated Bias in Total Pressure Results due to Turbulence

Results for dynamic pressure at off-calibration turbulence intensities are shown in Figure 4.38. Detailed results at $12 \%$ are also included in Figure 4.39. These results have three distinct features: (1) an overestimation of dynamic pressure for most flow angles when high-turbulence data were reduced using a low turbulence calibration; (2) an increase in $\Delta C_{q}$ near $\theta \approx 17^{\circ}$ for results at elevated turbulence intensities; and (3) a 
negative slope in the results for the $8 \%$ and $12 \%$ validation data sets. These three features were found in all off-calibration results for dynamic pressure regardless of the Reynolds number investigated or the seven-hole probe used.

The overestimation of dynamic pressure at low flow angles is in agreement with previous work by Takahashi (1997). Takahashi also found that seven-hole probes calibrated at low-turbulence conditions overestimated the dynamic pressure in highturbulence flows. However, similar to the results presented for the three-hole probe, the values of $\Delta C_{q}$ shown here at higher turbulence intensities include a significant uncertainty due to the uncertainty in the value of the true dynamic pressure $C_{q}$ Cal . This uncertainty is described briefly below and in full detail in Section 4.4.2.

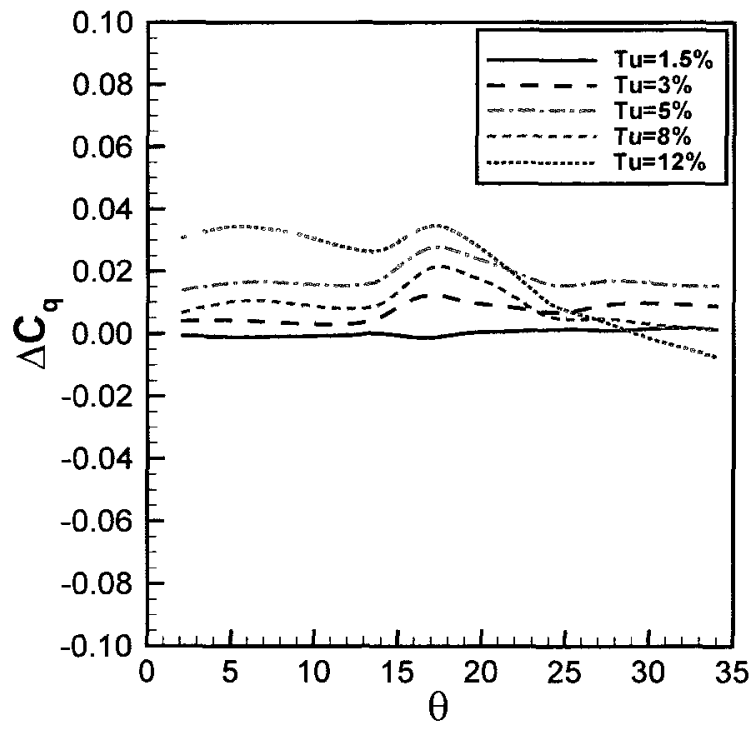

Figure 4.38: Seven-Hole Pressure Probe Dynamic Pressure Error due to Turbulence Intensity using a Calibration at $\operatorname{Re}_{\mathrm{D}}=3900, \mathrm{Tu}=1.5 \%$ 


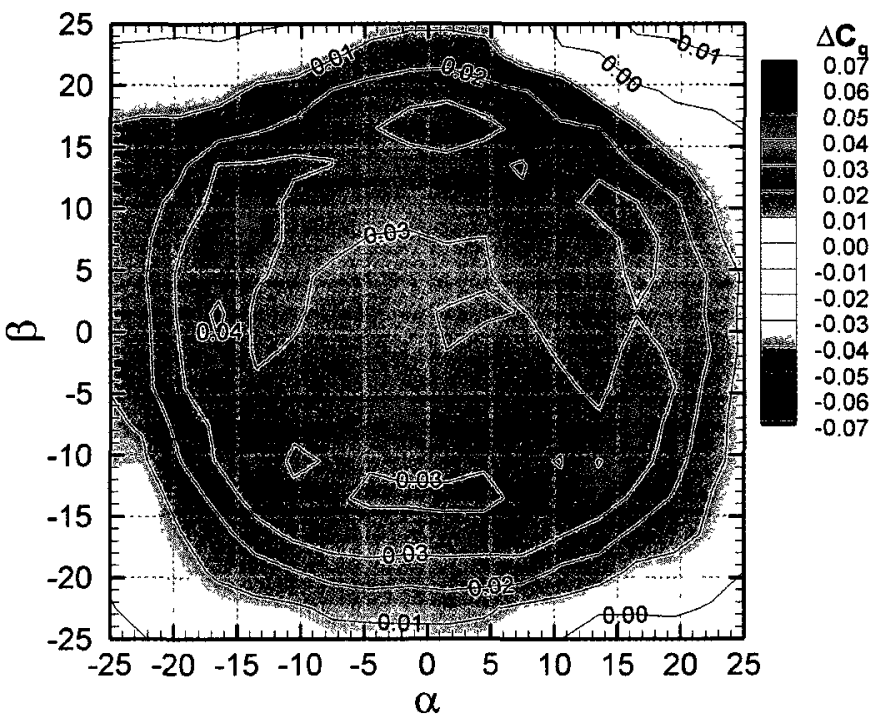

Figure 4.39: Seven-Hole Pressure Probe Dynamic Pressure Error due to Turbulence Intensity using a Calibration at $\operatorname{Re}_{\mathrm{D}}=3900, \mathrm{Tu}=1.5 \%$ and Validation Data at $\operatorname{Re}_{\mathrm{D}}=3900$, $\mathrm{Tu}=12 \%$

$\Delta C_{q}$ is defined as:

$$
\Delta C_{q}=\frac{q_{\text {Inferred }}-q_{\text {True }}}{q_{\text {True }}}
$$

and it compares the value of dynamic pressure inferred from the probe measurements to the true value. Thus, uncertainty in $q_{\text {True }}$ directly influences the results for $\Delta C_{q}$ shown here. The value of true dynamic pressure was determined for each test using the coefficient $C_{q C a l}$, defined as:

$$
C_{q \mathrm{Cal}}=\frac{q_{\text {rrue }}}{P_{c 1}-P_{c 2}}
$$


where $P_{c 1}$ and $P_{c 2}$ are the reference total and static pressures on the calibration rig. $C_{q C a l}$ was determined by finding $q_{\text {True }}$ for each operating point using pressure and hot-wire measurements obtained prior to data collection with the seven-hole probe. Table 4.5, repeated below as Table 4.13 , shows the values of $C_{q C a l}$ determined for each turbulence intensity and their estimated uncertainties. These values of $C_{q \mathrm{Cal}}$ were found to be independent of the Reynolds number of the flow but varied with turbulence intensity.

\begin{tabular}{|c|c|}
\hline$T u(\%)$ & $\mathrm{C}_{\mathrm{qCal}}$ \\
\hline 1.5 & $0.992 \pm 0.005$ \\
\hline 3 & $0.987 \pm 0.010$ \\
\hline 5 & $0.932 \pm 0.047$ \\
\hline 8 & $0.581 \pm 0.029$ \\
\hline 12 & $0.452 \pm 0.023$ \\
\hline
\end{tabular}

Table 4.13: Values of $\mathrm{C}_{\mathrm{q} C a l}$ for Different Turbulence Intensities

The uncertainty in $C_{q C a l}$ is significant for data collected at resultant turbulence intensities of $5 \%$ or greater. To quantify the effect this has on results, Figure 4.40 shows the $12 \%$ data reduced using the minimum, nominal, and maximum values quoted for $C_{q C a l}$. Because the same value of $C_{q C a l}$ applies to all data at a given turbulence intensity, error in this value results in a bias error and does not change the shape of the curves shown. Thus, although the magnitude of $\Delta C_{q}$ at any given flow angle is uncertain, the trends in the curves are not. This means that the rise in $\Delta C_{q}$ near $\theta \approx 17^{\circ}$ and the negative slopes in the high-turbulence results in Figure 4.38 not a result of this uncertainty and require further explanation. 


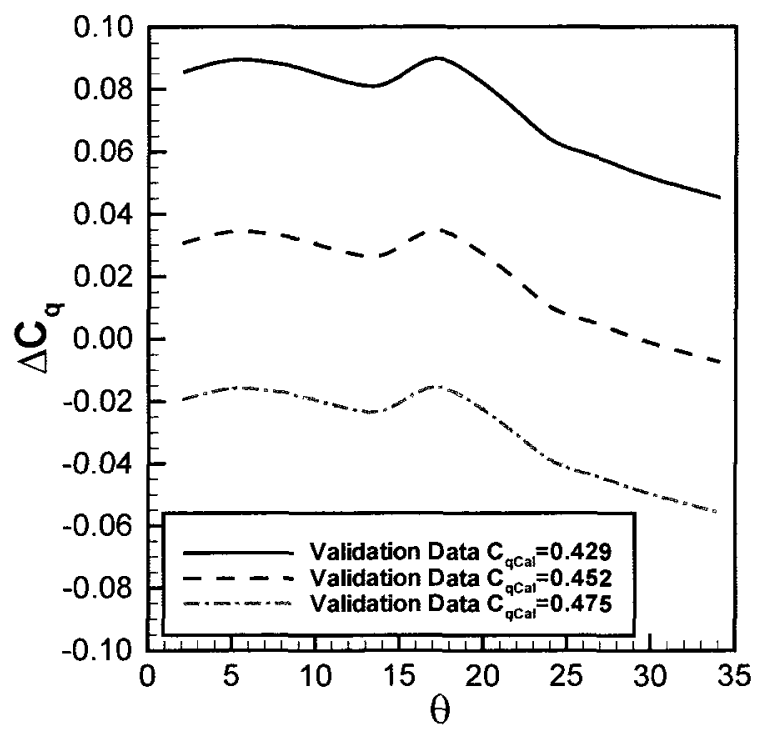

Figure 4.40: Effect of Uncertainty in $\mathrm{C}_{\mathrm{q} \text { Cal }}$ on Seven-Hole Probe Dynamic Pressure Results using a Calibration at $\operatorname{Re}_{\mathrm{D}}=3900, \mathrm{Tu}=1.5 \%$ and Validation data $\operatorname{Re}_{\mathrm{D}}=3900, \mathrm{Tu}=12 \%$

As discussed previously in Section 4.4.3, Johnson (1985) found through flow visualization that five-hole probes inclined relative to a flow were prone to the flow separating from their leeward side. This flow separation began at the tip of the probe and, depending on the inclination angle, could extend over the peripheral hole on the leeward side. Dominy and Hodson (1993) found that the presence of this separation affected the calibration coefficients for the probe and that because turbulence intensity can influence the conditions at which a flow will separate from a body, turbulence intensity thus influenced the probe calibration through this separation.

The change in $\Delta C_{q}$ near $\theta \approx 17^{\circ}$ in Figure 4.38 may be the result of a separation similar to that described by Johnson (1985) and Dominy and Hodson (1993). If the flow is separating from the seven-hole probe in low-turbulence flows but not in high- 
turbulence flows, this should be reflected in the shape of the calibration surfaces at the different turbulence conditions. Figure 4.41 shows the value of the dynamic pressure calibration coefficient for the seven-hole probe at the five turbulence intensities: the two plots included show cut-planes of the $C_{q 7 h p}$ surface along $\beta=0$ and $\alpha=0$, where $C_{q 7 h p}$ was previously defined in Equation 3.26 as:

$$
C_{q 7 h p}=\frac{P_{7}-\overline{P_{1-6}}}{P_{o}-P}
$$

$P_{o}$ is the total pressure, $P$ is the static pressure, $\overline{P_{1-6}}$ is the mean of the pressures at ports 1 through 6 , and $P_{7}$ is the pressure at the centre port.

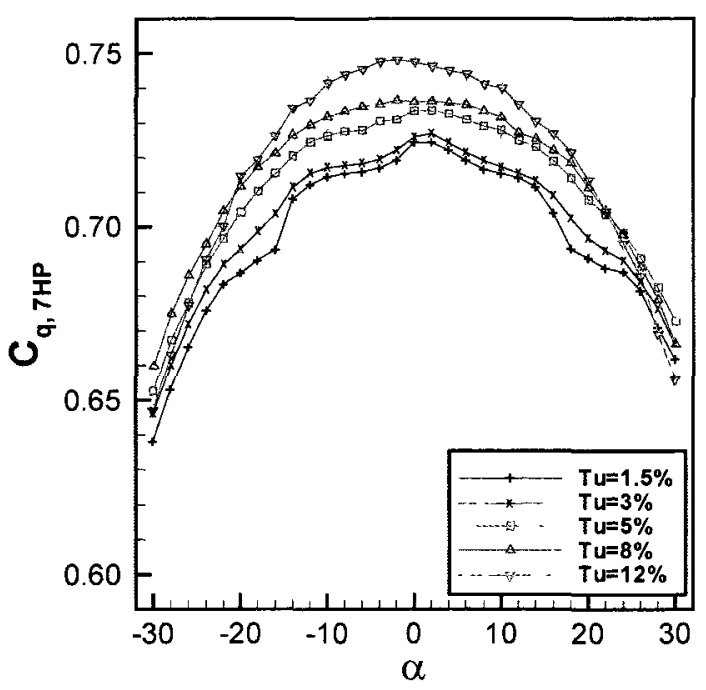

a) Cut-plane at $\beta=0$

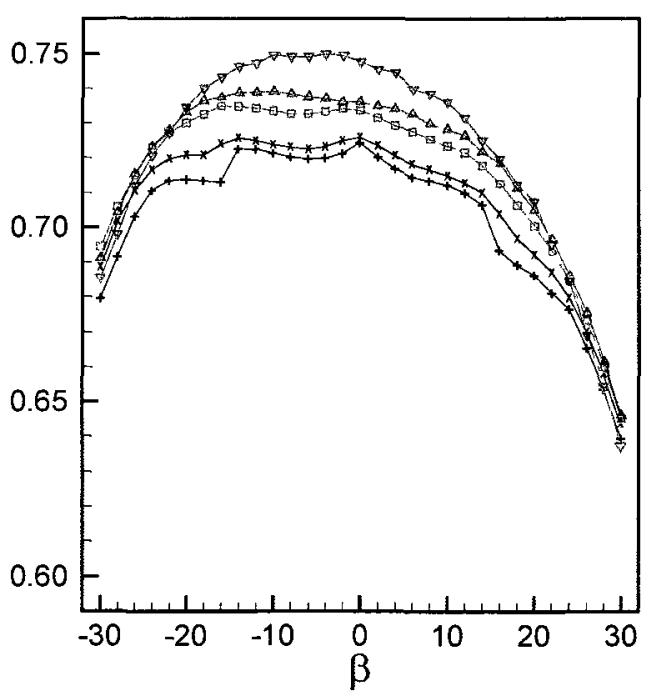

b) Cut-plane at $\alpha=0$

Figure 4.41: Cut-Planes of the Dynamic Pressure Calibration Coefficient for the Seven-Hole Pressure Probe at a Reynolds Number of 3900 and Various Turbulence Intensities 
Figure 4.41 shows that the values of $C_{q 7 h p}$ at turbulence intensities of $3 \%$ or greater are offset from one another but follow similar, relatively smooth, curves. The curve for $1.5 \%$ however includes a discontinuity near $\theta \approx 17^{\circ}$ that is not present at the other operating points. The response at $1.5 \%$ is likely an indication of a flow separation on the tip of the probe that is not present for the other turbulence intensities. This conclusion is speculative, however, as no direct evidence of a flow separation, such as flow visualization, has been collected here. Provided it is correct, this conclusion is also only applicable to seven-hole probes with the same cone angle as the probe used here $\left(60^{\circ}\right)$ and a similarly sharp probe leading edge; flows over probes with different geometries may separate but these separations would likely occur at different misalignments angles.

Finally, apart from the rise in $\Delta C_{q}$ at $\theta \approx 17^{\circ}$, the trends in the results for the $8 \%$ and $12 \%$ data in Figure 4.38 show that $\Delta C_{q}$ decreases with increasing $\theta$. This means that, regardless of the actual value of $\Delta C_{q}$ at $\theta=0^{\circ}$, the accuracy of the probe changes with increasing flow angle. This trend is in general agreement with the results previously discussed for the three-hole probe.

\subsubsection{Effects of Reynolds Number on Seven-Hole Pressure Probe Flow Measurements at Off-Calibration Conditions}

Results are presented below for data reduction at off-calibration Reynolds numbers: data collected at three Reynolds numbers were reduced using a calibration at a Reynolds number of 5150. Results shown in the main text use trend lines for clarity but full scatter plots are included in Appendix D. 
Results for flow angle are shown in Figure 4.42. For angles less than about $25^{\circ}$, reducing the data at off-calibration Reynolds numbers resulted in no notable effect: all results were within the uncertainty for the measurements. A small overestimation of flow angle is observed for flow angles greater than about $25^{\circ}$. These angles are outside the region typically considered to be the low-angle regime and thus this is not unexpected since the flow is likely separated from one or more of the pressure ports on the leeward side of the probe.

Trend lines showing the results for total pressure coefficient $\Delta C_{P o}$ at off-calibration Reynolds numbers are given in Figure 4.43; Figure 4.44 shows the results for the validation data collected $R e_{D}=2500$ using a colourflood contour plot. These results show that Reynolds number has little influence on the measurement of total pressure,

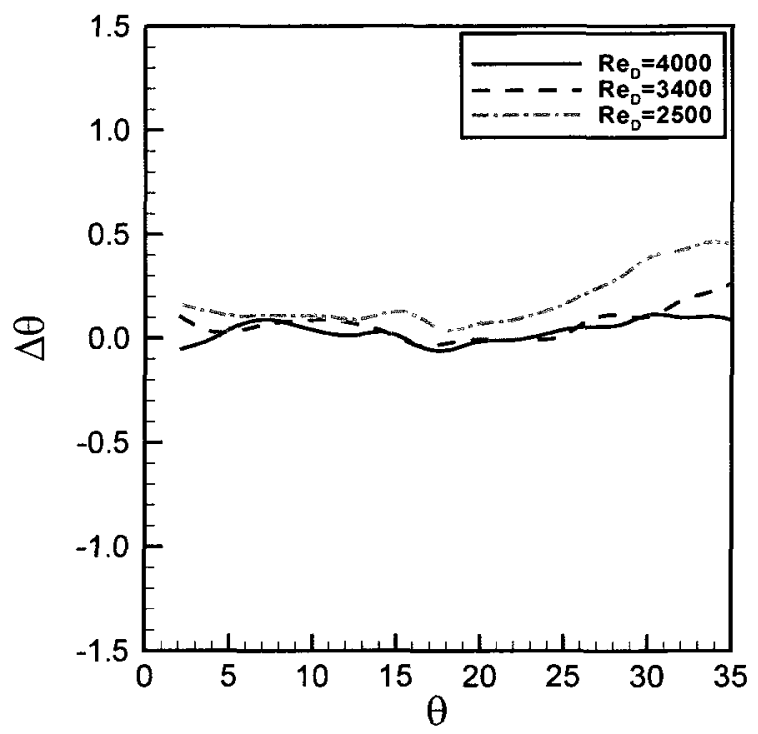

Figure 4.42: Seven-Hole Pressure Probe Flow Angle Error due to Reynolds Number using a Calibration at $\operatorname{Re}_{\mathrm{D}}=\mathbf{5 1 5 0}, \mathrm{Tu}=\mathbf{1 . 5 \%}$ 
especially for small flow angles. Results collected at flow angles of less than about 10$15^{\circ}$ for all Reynolds numbers investigated here were within the uncertainty in the measurements. For larger flow angles, reducing data using a calibration at a higher Reynolds number resulted in a small underestimation of total pressure. These results are similar to the trends previously discussed for the three-hole probe and also in general agreement with discussion from MacIsaac et al. (2009). MacIsaac et al. found that Reynolds number had a small effect on the total pressure calibration coefficient for the seven-hole probe at large angles but that this effect was negligible at small angles.

Figure 4.45 presents results for dynamic pressure at off-calibration Reynolds numbers. A colourflood contour plot for the results at $R e_{D}=2500$ is also included below as Figure 4.46. Both figures show that the measurement of dynamic pressure is quite sensitive to the Reynolds number of the calibration: reducing data using a calibration that was collected at a higher Reynolds number results in an underestimation of dynamic pressure at all flow angles. This underestimation was relatively constant for a given Reynolds number over flow angles of $\theta<15^{\circ}$ and $\theta>25^{\circ}$ but increased over the range of $15^{\circ}<\theta<25^{\circ}$.

At low and high flow angles, the response of the seven-hole probe to off-calibration Reynolds numbers was very similar to that of the three-hole probe. Data reduction with the three-hole probe at off-calibration Reynolds numbers resulted in a constant bias error in the values of $\Delta C_{q}$ at all flow angles. The seven-hole probe response is also in 


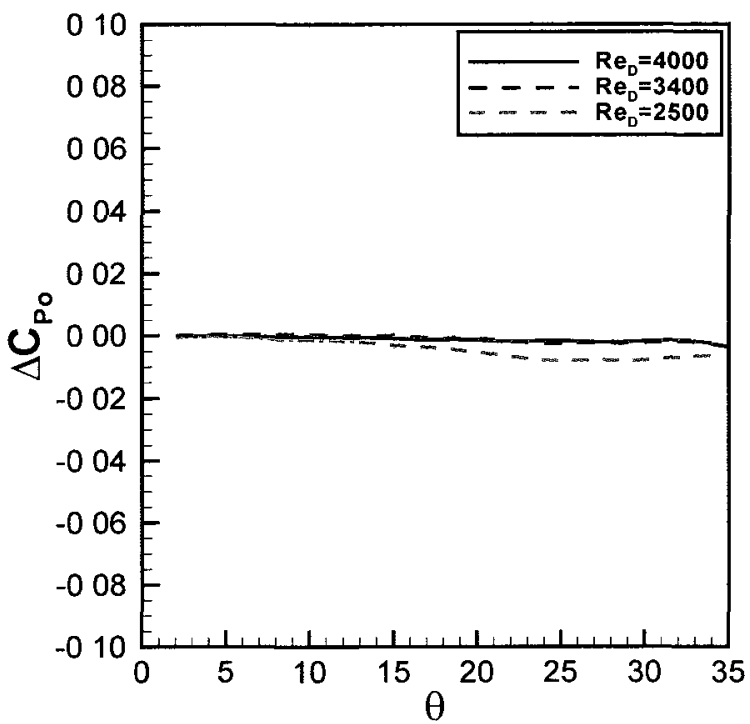

Figure 4.43: Seven-Hole Pressure Probe Total Pressure Error due to Reynolds Number using a Calibration at $\operatorname{Re}_{\mathrm{D}}=\mathbf{5 1 5 0}, \mathrm{Tu}=\mathbf{1 . 5 \%}$

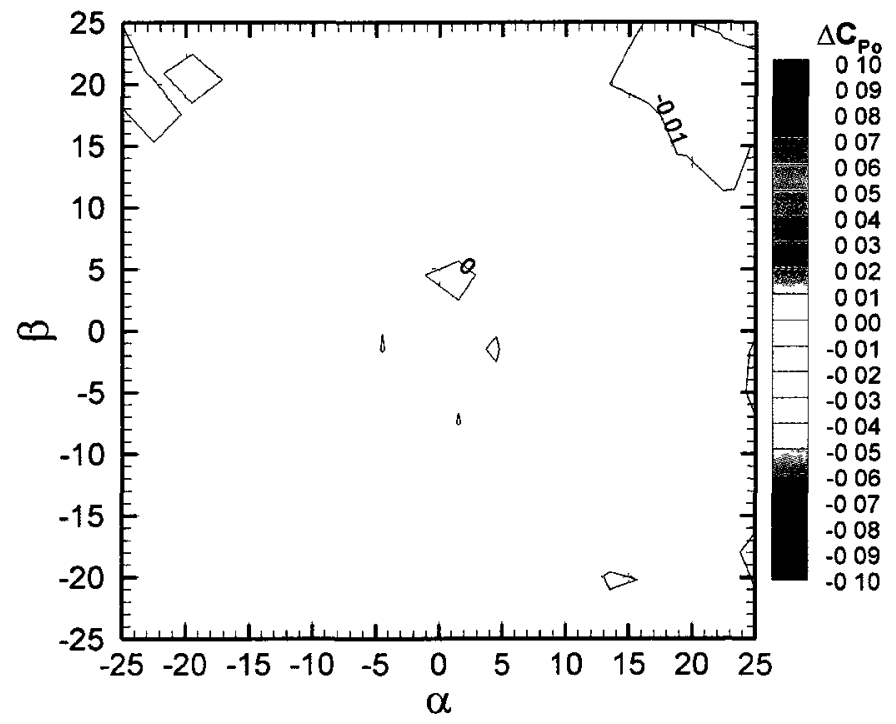

Figure 4.44: Seven-Hole Pressure Probe Total Pressure Error due to Reynolds Number using a Calibration at $R e_{D}=5150, T u=1.5 \%$ and Validation Data at $R e_{D}=2500, T u=1.5 \%$ 


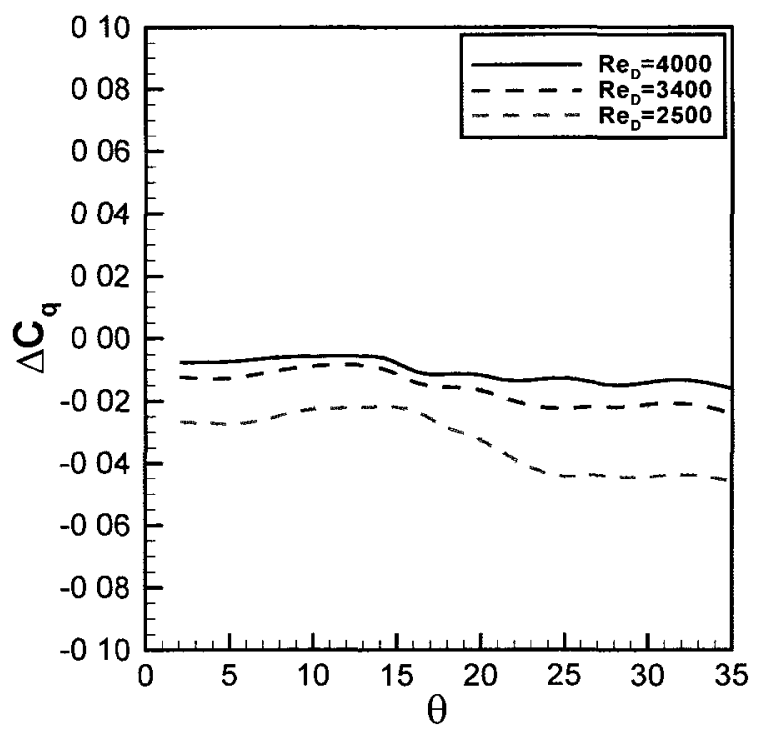

Figure 4.45: Seven-Hole Pressure Probe Dynamic Pressure Error due to Reynolds Number using a Calibration at $\operatorname{Re}_{\mathrm{D}}=\mathbf{5 1 5 0}, \mathrm{Tu}=\mathbf{1 . 5 \%}$

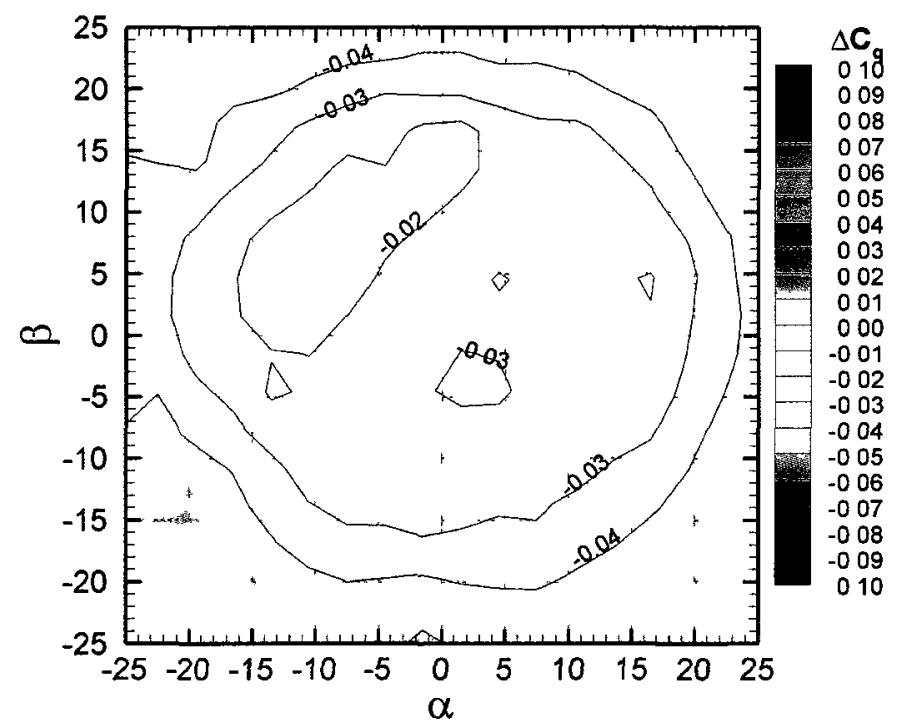

Figure 4.46: Seven-Hole Pressure Probe Dynamic Pressure Error due to Reynolds Number using a Calibration at $\operatorname{Re}_{\mathrm{D}}=\mathbf{5 1 5 0}, \mathrm{T} u=1.5 \%$ and Validation Data at $\operatorname{Re}_{\mathrm{D}}=2500, T u=1.5 \%$ 
agreement with previous seven-hole probe research by MacIsaac et al. (2009) and Wenger and Devenport (1999). MacIsaac et al. found that changes in Reynolds number caused a bias in the dynamic pressure coefficient for the seven-hole probe similar to the bias observed in the present study. Additionally, Wenger and Davenport found that reducing aligned seven-hole probe data $\left(\theta=0^{\circ}\right)$ collected at a Reynolds number of 2500 using a calibration at 3900 resulted in velocity being underestimated by about $1 \%$. Although omitted from the main text here, the present author examined a similar case using a calibration collected at $\mathrm{Re}_{D}=4000$ to reduce data at 2500 and found dynamic pressure was underestimated by about $2 \%$. This equates to an underestimation of velocity of about $1 \%$ and thus is in close agreement with results from Wenger and Davenport.

Figure 4.47 plots the values of $\Delta C_{q}$ for the seven-hole probe at $\theta \approx 0^{\circ}$ and $\theta=25^{\circ}$ as a function of the Reynolds number of the validation data. It should be noted that although validation data were not collected at the same Reynolds number as the calibration, $R e_{D}=5150$, it is reasonable to assume that $\Delta C_{q} \approx 0$ when $R e_{\text {Calbraton }}=R e_{\text {Valdatton }}$ and thus this point has been added to both curves. This figure shows that, as Reynolds number decreases, the effect of Reynolds number on data reduction increases. This is to be expected as viscous forces have a greater influence relative to inertial forces at lower Reynolds numbers. The shape of these curves also indicates that the influence of Reynolds number on the seven-hole probe used here is minimal for Reynolds numbers greater than about 5500 . 
In contrast with results for the three-hole probe, probe response for the seven-hole probe at moderate flow angles $15^{\circ}<\theta<25^{\circ}$ varied with flow angle. One possible explanation for this is the influence of a flow separation on the leeward side of the tip of the probe. As discussed previously in several sections, Johnson (1985) and Dominy and Hodson (1993) found that flow separation on the tips of five-hole probes affected the probe calibration. These separations, and thus the probe calibrations, were influenced by the probe Reynolds number.

\subsubsection{Summary}

The effects of turbulence intensity and Reynolds number on the seven-hole pressure probe are summarized qualitatively in Tables 4.14 and 4.15 . These tables include a summary of the results obtained using the high-turbulence and low-Reynolds number

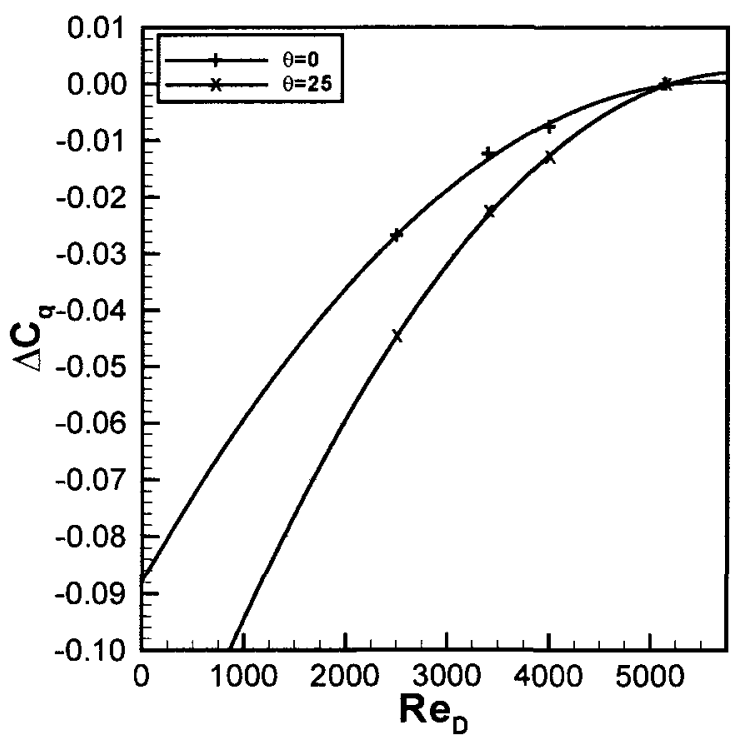

Figure 4.47: Seven-Hole Pressure Probe Dynamic Pressure Error due to Reynolds Number using a Calibration at $R e_{D}=5150$ and $T u=1.5 \%$ 
calibrations in Appendix D and the precision errors as discussed in Section 3.4.4.

\begin{tabular}{|c|c|c|c|}
\hline & \multicolumn{2}{|c|}{ Validation Data at: } \\
\hline & & Low Turbulence & High Turbulence \\
\hline \multirow{2}{*}{ 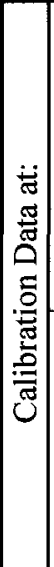 } & 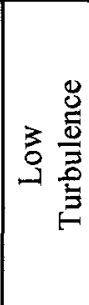 & $\begin{array}{l}\text { Precision Error: minimal } \\
\theta: \text { no systematic error } \\
\boldsymbol{P}_{o}: \text { no systematic error } \\
q: \text { no systematic error }\end{array}$ & $\begin{array}{l}\text { Precision Error: significant } \\
\theta \text { : no systematic error } \\
\text { Po: minimal error at } \theta=0^{\circ} \text {, significant } \\
\text { underestimation that increases with } \theta \text { for } \\
\theta>0^{\circ} \\
q: \text { complex response, see Section } 4.5 .2\end{array}$ \\
\hline & 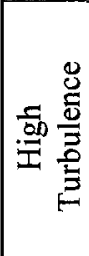 & $\begin{array}{l}\text { Precision Error: significant } \\
\theta: \text { no systematic error } \\
\boldsymbol{P}_{o}: \text { minimal error at } \theta=0^{\circ} \text {, significant } \\
\text { overestimation that increases with } \theta \text { for } \theta>0^{\circ} \\
q \text { : complex response, see Section } 4.5 .2\end{array}$ & $\begin{array}{l}\text { Precision Error: significant } \\
\boldsymbol{\theta}: \text { no systematic error } \\
\boldsymbol{P}_{o}: \text { no systematic error } \\
q \text { : no systematic error }\end{array}$ \\
\hline
\end{tabular}

Table 4.14: Summary of the Effects of Turbulence on Seven-Hole Pressure Probe

\section{Measurements}

\begin{tabular}{|c|c|c|c|}
\hline & \multicolumn{2}{|c|}{ Validation Data at: } \\
\hline & & Low Reynolds Number & High Reynolds Number \\
\hline \multirow{2}{*}{ 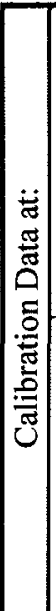 } & 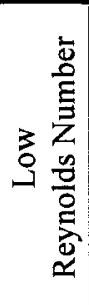 & $\begin{array}{l}\text { Precision Error: minimal } \\
\theta: \text { no systematic error } \\
P_{o}: \text { no systematic error } \\
q: \text { no systematic error }\end{array}$ & $\begin{array}{l}\text { Precision Error: minimal } \\
\theta \text { : no systematic error for } \theta<25^{\circ} \\
P o: \text { no error at } \theta=0^{\circ} \text {, very small } \\
\text { overestimation that increases with } \theta \text { for } \\
\theta \gg 0^{\circ} \text { and } R e_{C a l} \ll R e_{V a l} \\
q: \text { significant overestimation at all } \theta\end{array}$ \\
\hline & 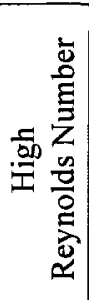 & $\begin{array}{l}\text { Precision Error: minimal } \\
\theta \text { : no systematic error for } \theta<25^{\circ} \\
\boldsymbol{P}_{v} \text { : no error at } \theta=0^{\circ} \text {, very small } \\
\text { underestimation that increases with } \theta \text { for } \\
\theta \gg 0^{\circ} \text { and } \operatorname{Re} e_{C a l} \gg \operatorname{Re}_{V a l} \\
q \text { : significant underestimation at all } \theta\end{array}$ & $\begin{array}{l}\text { Precision Error: minimal } \\
\theta: \text { no systematic error } \\
P_{o}: \text { no systematic error } \\
q: \text { no systematic error }\end{array}$ \\
\hline
\end{tabular}

Table 4.15: Summary of the Effects of Reynolds Number on Seven-Hole Pressure Probe Measurements 


\subsection{Method to Account for the Effects of Turbulence Intensity and Reynolds Number on Seven-Hole Pressure Probe Data Reduction}

Seven-hole pressure probes have been used extensively at Carleton University to make detailed flow field measurements in low-speed linear turbine cascades. Examples of these measurements include results presented by Benner et al. (2004), Knezevici et al. (2010) and MacIsaac et al. (2009). The standard procedure for reducing seven-hole probe data in recent studies has been to calibrate the probe at several Reynolds numbers and interpolate between these calibrations to account for the effects of Reynolds number on data reduction. This procedure is explained in more detail by MacIsaac et al. To account for the effects of both Reynolds number and turbulence intensity on results, the present author has extended the data reduction method of MacIsaac et al. to a twodimensional interpolation on both flow parameters. This new technique has the added requirement that the turbulence intensity at each data point be measured prior to data reduction, for example using a hot-wire probe, but ensures that the effects of Reynolds number and turbulence intensity are both accounted for as accurately as possible.

The following section revisits the results for total pressure collected by MacIsaac et al. in a low-speed linear cascade. The authors used the same seven-hole probe as discussed in Section 4.5 (7HP1) to obtain data in a three-dimensional flow field with probe Reynolds numbers of 2500 to 4000 and resultant turbulence intensities of 1.5 to $9.5 \%$. The present author has reduced this seven-hole probe data using the calibrations 
collected here and two data reduction procedures: the procedure used by MacIsaac et al. that accounts for the effects of Reynolds number only, and the procedure proposed above that accounts for the effects of both Reynolds number and turbulence intensity. Table 4.16 lists the calibrations employed for this analysis: the Reynolds number interpolation used the calibrations taken at a turbulence intensity of $1.5 \%$, and the two-dimensional interpolation used calibrations at all turbulence intensities.

\begin{tabular}{|c|cc|c|c|c|c|c|}
\hline $\begin{array}{c}\mathrm{Re}_{\mathrm{D}} \\
\text { nominal] }\end{array}$ & \multicolumn{2}{|c|}{$\begin{array}{c}T u(\%) \\
\text { [nominal] }\end{array}$} & $\begin{array}{c}T u_{u} \\
(\%)\end{array}$ & $\begin{array}{c}T u_{v} \\
(\%)\end{array}$ & $\begin{array}{c}T u_{w} \\
(\%)\end{array}$ & $\begin{array}{c}\Lambda_{x} \\
(\mathrm{~mm})\end{array}$ \\
\hline 2500 & {$[2500]$} & 1.7 & {$[1.5]$} & 0.6 & 2.5 & 1.3 & - \\
\hline 3920 & {$[3900]$} & 1.7 & {$[1.5]$} & 0.6 & 2.4 & 1.7 & - \\
\hline 2490 & {$[2500]$} & 3.1 & {$[3]$} & 2.0 & 3.9 & 3.1 & 7.3 \\
\hline 3910 & {$[3900]$} & 2.9 & {$[3]$} & 2.0 & 3.6 & 2.9 & 5.6 \\
\hline 2490 & {$[2500]$} & 5.3 & {$[5]$} & 4.0 & 5.6 & 6.0 & 4.6 \\
\hline 3930 & {$[3900]$} & 5.0 & {$[5]$} & 3.7 & 5.4 & 5.6 & 4.0 \\
\hline 2470 & {$[2500]$} & 7.7 & {$[8]$} & 5.7 & 8.4 & 8.6 & 5.4 \\
\hline 3910 & {$[3900]$} & 8.1 & {$[8]$} & 5.7 & 9.0 & 9.2 & 5.3 \\
\hline 2470 & {$[2500]$} & 12.2 & {$[12]$} & 7.3 & 14.2 & 13.7 & 7.4 \\
\hline 3890 & {$[3900]$} & 12.4 & {$[12]$} & 7.3 & 14.7 & 14 & 7.5 \\
\hline
\end{tabular}

Table 4.16: Seven-Hole Pressure Probe Calibrations used to Reduce Data from MacIsaac et al. (2009)

Results for total pressure are presented below in terms of the total pressure coefficient, $C_{P o W T}$, defined as:

$$
C_{P_{o} W T}=\frac{P_{o}-P_{o r e f}}{q_{r e f}}
$$

where $P_{o}$ is the value of total pressure from the probe and $P_{\text {oref }}$ and $q_{\text {ref }}$ are upstream reference values of total and dynamic pressure. Figure $4.48 \mathrm{a}$ shows the results for total 
pressure obtained using the Reynolds number interpolation method at $T u=1.5 \%$; Figure 4.48b shows the results obtained using the two-dimensional interpolation on Reynolds number and turbulence intensity. Both figures present the distribution of the total pressure coefficient from a plane located at 1.4 axial chords downstream of the leading edge of the cascade. The measurements extended over half the blade $\operatorname{span}(z / h=0.0$ to 0.5$)$ and 1.2 blade spacings $(\Delta y / s=1.2)$.

Although the plots are qualitatively very similar, the local values of $C_{P o W T}$ in Figures $4.48 \mathrm{a}$ and $4.48 \mathrm{~b}$ differ by as much as $16 \%$ of the reference dynamic pressure in some regions of the flow. Figure 4.49 shows these differences using a point-by-point comparison defined by:

$$
\Delta C_{P_{o} W T}=\frac{P_{o 1.5 \%}-P_{o R e f}}{q_{\text {Ref }}}-\frac{P_{o A l l T u}-P_{o R e f}}{q_{R e f}}=C_{P_{o 1.5 \%}}-C_{P o A l l T u}
$$

The values of $\Delta C_{P O W T}$ are greater than the quoted uncertainty in the measurements of $\pm 0.3 \%$. According to discussion in Section 4.5 , these differences should be largest in regions of high resultant turbulence intensity $(T u)$ and high flow angle $(\theta)$. To highlight these areas, contours of a turbulence factor, $T F$, defined as:

$$
T F=\frac{T u}{T u_{\max }} \times \frac{\theta}{\theta_{\max }}
$$

are also included in Figure 4.49. As expected, the magnitude of $\Delta C_{P_{O} W T}$ is larger in regions of high $T F$. 


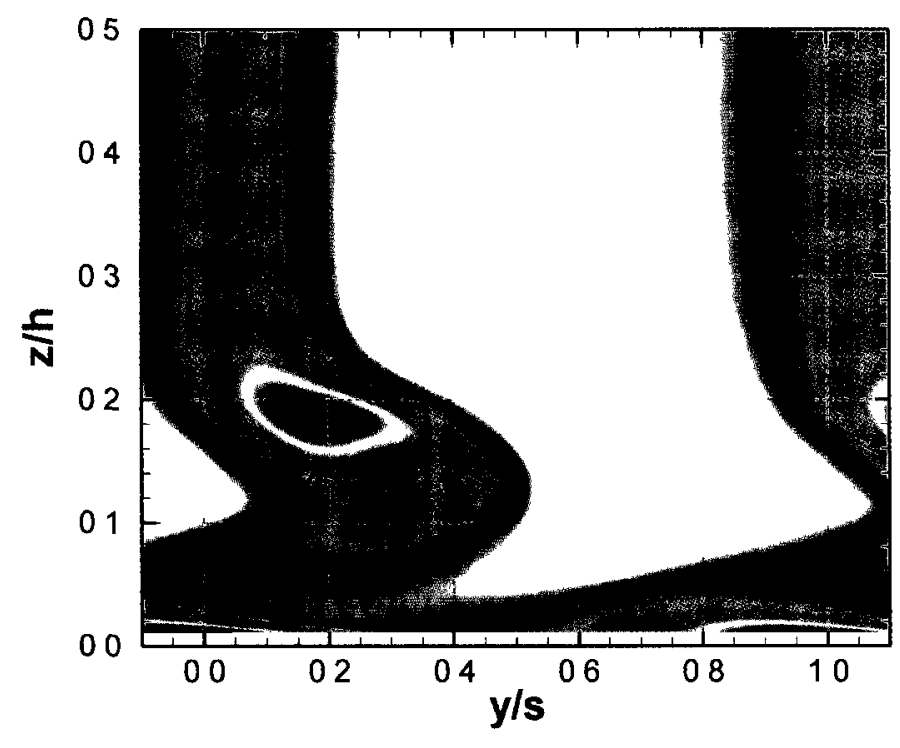

a) Reduced by Interpolating on Reynolds Number at $\mathrm{Tu}=1.5 \%$

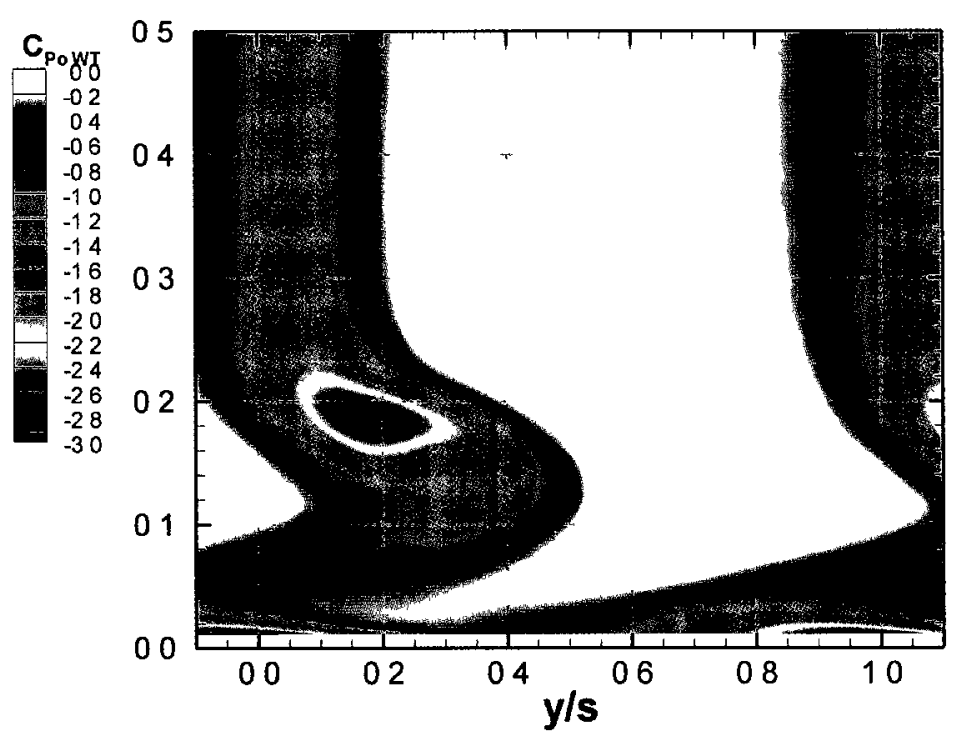

b) Reduced by Interpolating on Reynolds Number and Turbulence Intensity

Figure 4.48: Total Pressure Results using Seven-Hole Probe Data from MacIsaac et al. (2009) 


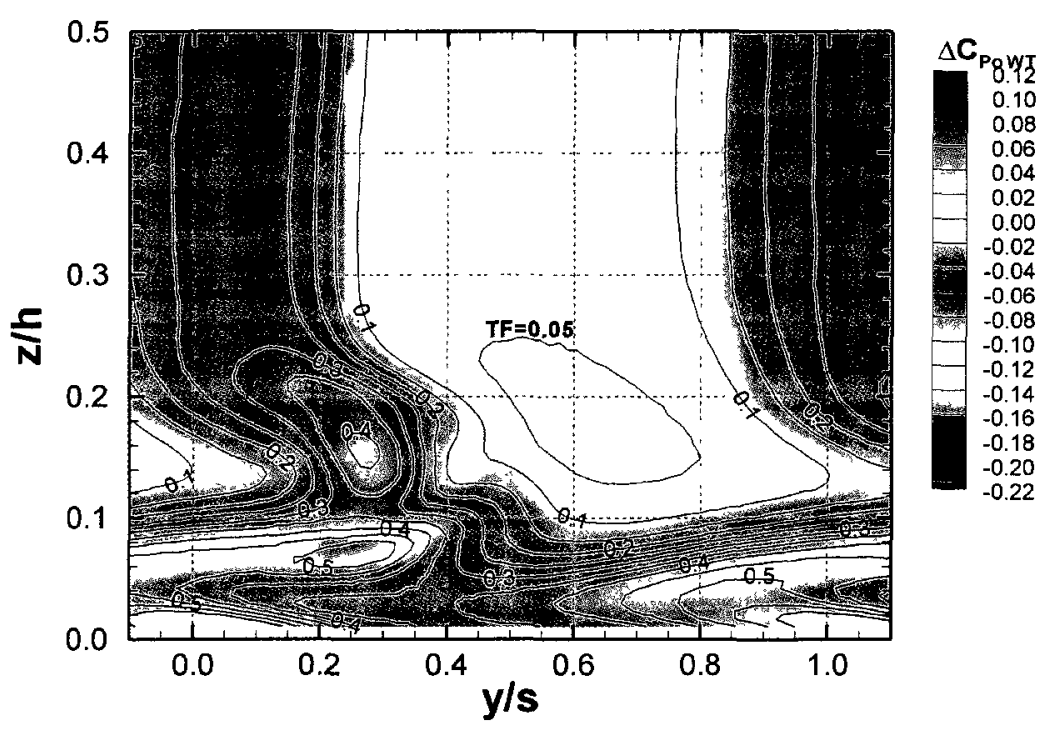

Figure 4.49: Comparison between the Results for Total Pressure from Interpolating on Reynolds Number at $\mathrm{Tu}=\mathbf{1 . 5 \%}$ and Interpolating on Both Turbulence Intensity and Reynolds Number

In addition to collecting seven-hole probe data, MacIsaac et al. also measured the total pressure in the same flow using a Kiel probe. As discussed by Chue (1975) (summarized in Chapter 2) and shown in Section 4.3, the Kiel probe is relatively insensitive to the effects of flow angle, turbulence intensity, and other flow parameters. Therefore, Kiel results should closely approximate the true values of total pressure in the flow. While results from the Kiel probe were qualitatively similar to those from the seven-hole probe, Figure 4.50 shows the differences between these results using:

$$
\Delta C_{P o W T}^{\prime}=C_{P o 7 h p}-C_{P o K i e l}
$$


Figure $4.50 \mathrm{a}$ presents this comparison with respect to the results from the interpolation on Reynolds number at $T u=1.5 \%$ (Figure $4.48 \mathrm{a}$ ); Figure $4.50 \mathrm{~b}$ with respect to the results from the interpolation on Reynolds number and turbulence intensity (Figure 4.48b).

Results produced by interpolating on Reynolds number only underestimate total pressure relative to the Kiel probe by as much as $21 \%$ of reference dynamic pressure. In contrast, results from interpolating on both Reynolds number and turbulence intensity differ from the Kiel values by less than $10 \%$. The two-dimensional interpolation did overestimate total pressure relative to the Kiel probe in some portions of the flow, but this overestimation was less than about $4 \%$ of reference dynamic pressure. This indicates that reducing the data by interpolating on Reynolds number and turbulence intensity provides the most accurate results for the total pressure.

Although accounting for the influence of turbulence intensity in addition to Reynolds number improved the agreement between the Kiel and seven-hole probe results, these results do not agree equally well in all regions of the flow. This is likely due in part to the influence of other factors, such as shear, vorticity, the isotropy of the turbulence, or the turbulent length scale, that have not been accounted for here. Furthermore, while comparing calibrations collected by the present study and MacIsaac et al. at similar operating points it became evident that the response of the seven-hole probe changed slightly between the studies. This change resulted in a systematic difference between the calibrations that was negligible at low flow angles but increased to $4 \%$ of the reference 


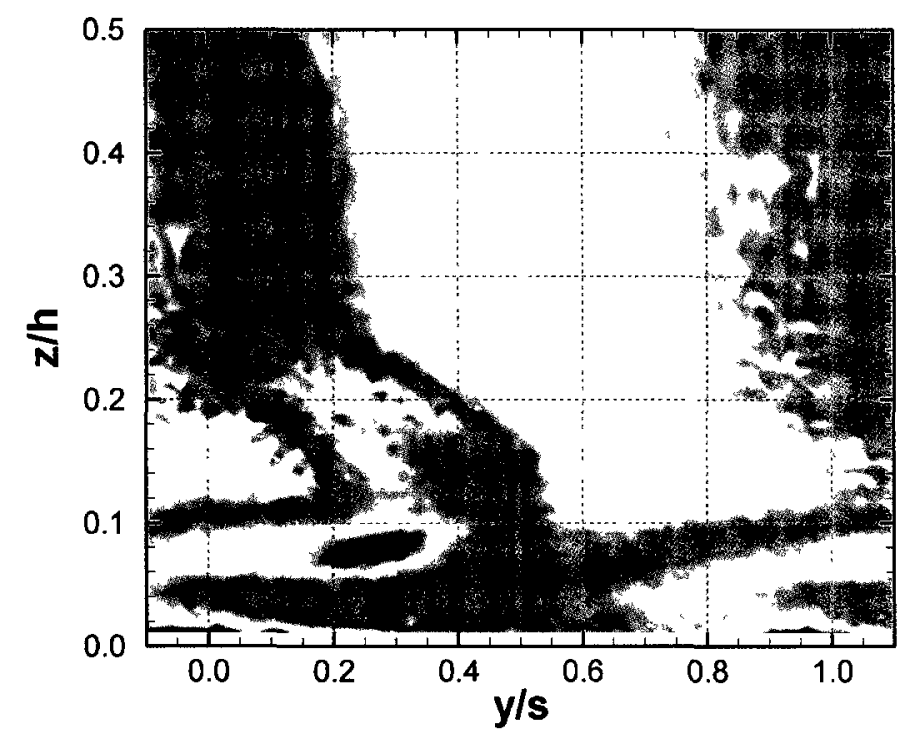

a) Seven-Hole Probe Data Reduced by Interpolating on Reynolds Number at Tu=1.5\%

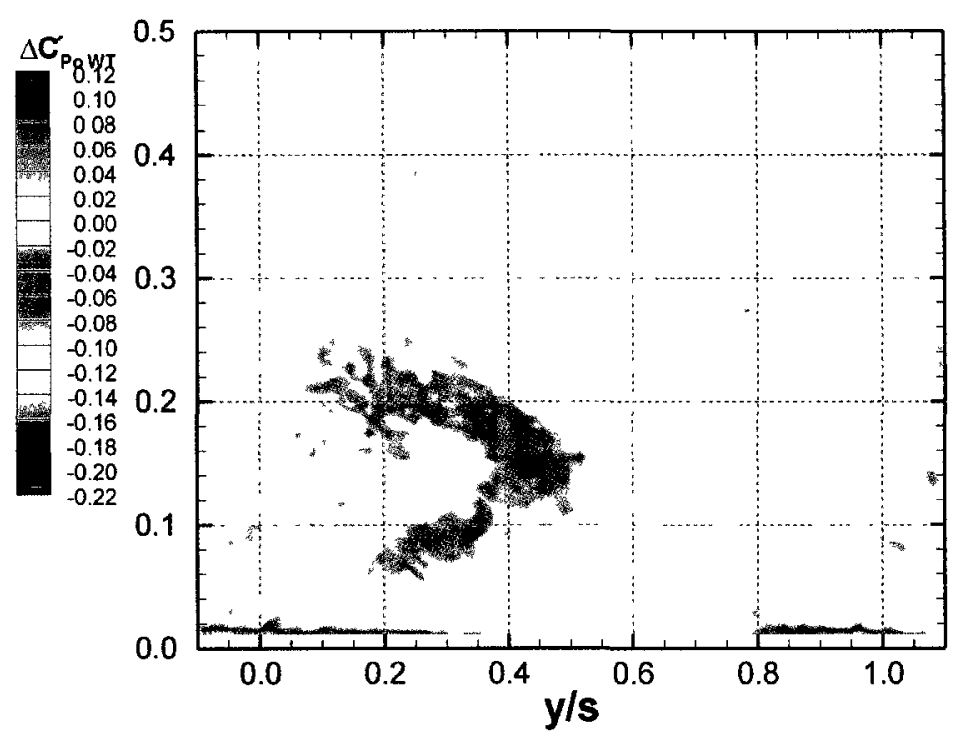

b) Seven-Hole Probe Data Reduced by Interpolating on Reynolds Number and Turbulence Intensity

Figure 4.50: Comparison of Total Pressure Results from the Seven-Hole and Kiel Probes 
dynamic pressure at the highest flow angles observed. The most likely cause of this change in probe behaviour is the fouling of the seven-hole probe described in Section 4.5.1. 


\section{Chapter 5}

\section{Conclusions and Recommendations}

\subsection{Conclusions}

The aerodynamic behaviour of pressure probes is influenced by the flows they measure. Examples of the flow parameters that influence these probes include the turbulence intensity, turbulent length scale, Reynolds number, shear, and Mach number. The present thesis investigated the effects of turbulence intensity and Reynolds number on the behaviour of the Kiel, three-hole, and seven-hole probes.

The quantification of these effects was conducted by collecting data with each probe over a range of turbulence intensities and Reynolds numbers and comparing the probeinferred results to the true values. To generate these flows, a calibration rig capable of producing flows with turbulence intensities of up to $12 \%$ was developed. This rig, which is believed to be of a novel design, combines the use of grids and a perforated pipe upstream of the measurement plane to generate the turbulence. The perforated pipe was preferred over the more traditional turbulence generating grids because, for a given turbulence intensity, the pipe caused lower pressure losses and thus allowed for higher 
flow velocities to be investigated. The turbulent flows generated by this setup were notably anisotropic: the streamwise components of the turbulence intensities were greater than the transverse components. This is believed to be a product of the flow acceleration through the nozzle immediately preceding the measurement plane and not a product of the turbulence generation method because all turbulent flows generated in the rig using either the pipe or grids had similar levels of anisotropy.

The present study found that the Kiel probe became more sensitive to flow misalignments at higher turbulence intensities. While Kiel measurements at turbulence intensities of $1.5 \%$ were insensitive to flow angles of less than about $34^{\circ}$, this angle decreased to about $24^{\circ}$ for data collected at an intensity of $12 \%$. These results were unaffected by the Reynolds number over the probe Reynolds number range of 1050 to 4350.

For the three- and seven-hole probes, both turbulence intensity and Reynolds number affected the calibration coefficients. These effects caused systematic errors in the results for total and dynamic pressure when data were reduced at off-calibration conditions. For total pressure, the errors due to both parameters were negligible for aligned measurements but increased with increasing flow angle. For dynamic pressure, Reynolds number caused a constant bias error in the results at all flow angles and turbulence intensity caused an error that varied with flow angle. The exact magnitude of the error in dynamic pressure due to turbulence intensity was uncertain, however, due to uncertainties in the value of the true dynamic pressure in the flow. The only notable difference 
between the responses of the two probes was in the results for flow angle. Both turbulence intensity and Reynolds number affected the results for flow angle from the three-hole probe but neither affected the results for flow angle from the seven-hole probe.

Lastly, a new data reduction procedure for the seven-hole probe was developed to account for the effects of turbulence intensity and Reynolds number on the calibration coefficients. This procedure, which used a two-dimensional interpolation between calibrations collected at different turbulence intensities and Reynolds numbers, was found to improve the accuracy of results from the seven-hole probe and should be applicable to other multi-hole probes. The main drawbacks to this procedure are that the user must calibrate their multi-hole probe at several turbulence intensities prior to data collection and that the turbulence intensity of the flow being investigated must be measured separately prior to reducing the multi-hole probe data.

\subsection{Recommendations for Future Work}

The present study clearly demonstrates that turbulence intensity affects the results for dynamic pressure obtained using the three- and seven-hole pressure probes. At high turbulence operating points, however, the nature of this effect relative to the true values could not be determined due to experimental uncertainties in the true dynamic pressure. Thus, it is recommended that future investigations of this nature use a more accurate method to determine the true dynamic pressure of the high-turbulence flows so that more specific conclusions may be drawn. 
In addition to the effects of turbulence intensity and Reynolds number, it is recognized that other flow parameters may influence probe behaviour. For example, the turbulent length scale and isotropy of the turbulent flow may also affect these probes. Further investigation into the effects of these parameters would be useful to improve the accuracy of the current data reduction techniques. It is, however, acknowledged that the experimental study of these parameters may be challenging because it is difficult to decouple the turbulence intensity from the turbulent length scale or to control the isotropy of the turbulent flow.

The calibration rig used here was sufficient for the present thesis, but improvements to this rig could make it more useful for future research. For instance, modifications to the rig may improve the isotropy of the turbulent flow. As the flow acceleration through the nozzle ahead of the measurement plane is believed to be the cause of the anisotropy in the flow, revising the rig design to remove this nozzle may result in more isotropic flow. Furthermore, if the change or removal of the nozzle alters the isotropy of the turbulence in the flow, this may yield a method by which the effect of isotropy on probe behaviour can be investigated. The use of different sized holes in the perforated pipe, or different diameters of pipe altogether, could also be investigated with the aim of obtaining different turbulence intensities and length scales in the flow. 


\section{References}

Barker, K. W., Gallington, R. W., and Minster, S. N. (1979), "Calibration of Five-hole Probes for On-line Data Reduction," Aeronautics Digest, USAFA-TR-79-7, USAF Academy.

Becker, H. A., and Brown, A. P. G. (1974), "Response of Pitot Probes in Turbulent Streams," Journal of Fluid Mechanics, Vol. 62, pp. 85-114.

Benner, M. W. (2003), "The Influence of Leading-Edge Geometry on Profile and Secondary Losses in Turbine Cascades," Ph.D. Thesis, Department of Mechanical \& Aerospace Engineering, Carleton University, Ottawa, Canada.

Benner, M. W., Sjolander, S. A., and Moustapha, S. H. (2004), "The Influence of Leading-Edge Geometry on Secondary Losses in a Turbine Cascade at the Design Incidence," Journal of Turbomachinery, Vol. 126, pp. 277-287.

Blake, W. K. (1983), "Differential Pressure Measurement." Fluid Mechanics Measurement. R. J. Goldstein. New York, Hemisphere Publishing Corporation: pp. 72.

Bruun, H. H. (1995), Hot-Wire Anemometry: Principles and Signal Analysis, Oxford University Press, New York.

Buresti, G., and Di Cocco, N. R. (1987), "Hot-wire Measurement Procedures and their Appraisal through a Simulation Technique," J. Phys. E: Sci. Instrum., Vol. 20, pp. 8799. 
Chernoray, V., and Hjarne, J. (2008), "Improving the Accuracy of Multihole Probe Measurements in Velocity Gradients." ASME Turbo Expo, Berlin, Germany, ASME.

Cho, S. H., and Becker, H. A. (1985), "Response of Static Pressure Probes in Turbulent Streams," Experiments in Fluids, Vol. 3, pp. 93-102.

Christiansen, T., and Bradshaw, P. (1981), "Effect of Turbulence on Pressure Probes," Journal of Physics E: Scientific Instruments, Vol. 14, pp. 992.

Chue, S. H. (1975), "Pressure Probes for Fluid Measurement," Progress in Aerospace Sciences, Vol. 16, pp. 147-223.

DeCecco, S. (1995), "Behavior of Tip-Leakage Flows at Large Clearances," M.A.Sc. Thesis, Department of Mechanical \& Aerospace Engineering, Carleton University, Ottawa, Canada.

Döbbeling, K., Lenze, B., and Leuckel, W. (1990), "Computer-aided Calibration and Measurements with a Quadruple Hotwire Probe," Experiments in Fluids, Vol. 8, pp. 257-262.

Dominy, R. G., and Hodson, H. P. (1993), "An Investigation of Factors Influencing the Calibration of Five-Hole Probes for Three-Dimensional Flow Measurements," Journal of Turbomachinery, Vol. 115, pp. 513-519.

Ertunc, O., and Durst, F. (2008), "On the High Contraction Ratio Anomaly of Axisymmetric Contraction of Grid-Generated Turbulence," Physics of Fluids, Vol. 20, pp. 025103.

Everett, K. N., Gerner, A. A., and Durston, D. A. (1983), "Seven-Hole Cone Probes for High Angle Flow Measurement: Theory and Calibration," AIAA Journal, Vol. 21, pp. 992-998. 
Fransson, J. H. M., Matsubara, M., and Alfredsson, P. H. (2005), "Transition Induced by Free-Stream Turbulence," Journal of Fluid Mechanics, Vol. 527, pp. 1-25.

Gad-El-Hak, M., and Corrsin, S. (1974), "Measurements of the Nearly Isotropic Turbulence Behind a Uniform Jet Grid," Journal of Fluid Mechanics, Vol. 62, pp. $115-143$

Gallington, R. W. (1980), "Measurement of Very Large Flow Angles with Non-Nulling Seven-Hole Probes," USAFA paper TR-80-17.

Gerner, A. A., Maurer, C. L., and Gallington, R. W. (1984), "Non-Nulling Seven-Hole Probes for High Angle Flow Measurement," Experiments in Fluids, Vol. 2, pp. 95103.

Goldstein, S. (1936), "A Note on the Measurement of Total Head and Static Pressure in a Turbulent Stream." Proceedings of the Royal Society of London. Series A, Mathematical and Physical Sciences, The Royal Society, Vol. 155, pp. 570-575.

Gracey, W., Coletti, D. E., and Russell, W. R. (1951), "Wind Tunnel Investigations of a number of Total Pressure Tubes at High Angle of Attack - Supersonic Speeds," NACA TN 2261.

Hinze, J. O. (1975), Turbulence, 2nd edition, McGrad-Hill, New York.

Islam, A. M. T. (1999), "An Experimental and Computational Study of the Aerodynamics of Turbine Blades With Damage," Ph.D. Thesis, Department of Mechanical \& Aerospace Engineering, Carleton University, Ottawa, Canada.

Johnson, A. B. (1985), "Reynolds Number Dependence of 5-Hole 3-Dimensional Truncated Cone Probes," Project report, Part II, Engineering Tripos, Cambridge University. 
Knezevici, D. C., Sjolander, S. A., Praisner, T. J., Allen-Bradley, E., and Grover, E. A. (2010), "Measurements of Secondary Losses in a Turbine Cascade With the Implementation of Nonaxisymmetric Endwall Contouring," Journal of Turbomachinery, Vol. 132, pp. 011013.

Krause, L. N., and Gettelman, C. C. (1952), "Considerations Entering into the Selection of Probes for Pressure Measurement in Jet Engines." ISA Proc., Vol. 7, pp. 134.

Kurian, T., and Fransson, J. H. M. (2009), "Grid-Generated Turbulence Revisited," Fluid Dynamics Research, Vol. 41, pp. 021403.

Laws, E. M., and Livesey, J. L. (1978), "Flow Through Screens," Annual Review of Fluid Mechanics, Vol. 10, pp. 247-266.

Lewis, W. E. (1966), "Fixed-Direction Probes for Aerodynamic Measurements." Proceedings of the Institution of Mechanical Engineers, 180 Part 3J, pp. 141-151.

Ligrani, P. M., Singer, B. A., and Baun, L. R. (1989), "Spatial Resolution and Downwash Velocity Corrections for Multiple-Hole Pressure Probes in Complex Flows," Experiments in Fluids, Vol. 7, pp. 424-426.

MacIsaac, G. D. (2010), "Calibration and Data Reduction Methods for a X-Wire Hotwire Probe," private communication.

MacIsaac, G. D., Taremi, F., Knezevici, D. C., Scribner, C. A., and Sjolander, S. A. (2009), "Challenges in using Kiel and Seven-hole Pressure Probes in Highly ThreeDimensional Flows: Application to Turbumachinery Cascade Measurements." ISABE, Montreal, Canada.

MacMillan, F. A. (1954a), "Viscous Effects on Flattened Pitot Tubes at Low Speeds," Journal of the Royal Aeronautical Society, Vol. 58, pp. 570-572. 
MacMillan, F. A. (1954b), "Viscous Effects on Pitot Tubes at Low Speeds," Journal of the Royal Aeronautical Society, Vol. 58, pp. 570-572.

Mahallati, A. (2003), "Aerodynamics of a Low-Pressure Turbine Airfoil under Steady and Periodically Unsteady Conditions," Ph.D. Thesis, Department of Mechanical \& Aerospace Engineering, Carleton University, Ottawa, Canada.

Matsunuma, T., Hiroyuki, A., and Tsutsui, Y. (1999), "Influence of Turbulence Intensity on Annular Turbine Stator Aerodynamics at Low Reynolds Numbers." International Gas Turbine \& Aeroengine Congress \& Exhibition, Indianapolis, Indiana, ASME.

Ower, E., and Pankhurst, R. C. (1977), The Measurement of Air Flow, 5th edition, Pergamon, Oxford.

Pinker, R. A., and Herbert, M. V. (1967), "Pressure Loss Associated with Compressible Flow through Square-Mesh Wire Gauzes.," Journal of Mechanical Engineering Science, Vol. 9, pp. 11-23.

Pisterman, K. (2004), "Use of a Seven-Hole Pressure Probe in Highly Turbulent FlowFields," M.A.Sc. thesis, Virginia Polytechnic Institute and State University, Blacksburg, United States of America.

Roach, P. E. (1987), "The Generation of Nearly Isotropic Turbulence by means of Grids," International Journal of Heat and Fluid Flow, Vol. 8, pp. 82-92.

Rossow, V. J. (1991), Probe Shapes for Streamwise Momentum and Cross-stream Turbulence Intensity, American Institute of Aeronautics and Astronautics, Reston, VA.

Schulze, W. M., Ashby, G. C., Jr., and Erwin, J. R. (1952), "Several Combination Probes for Surveying Static and Total Pressure and Flow Direction," NACA TN 2830. 
Sieverding, C. (1975), "Modern Methods of Testing Rotating Components of Turbomachines (Instrumentation)," North Atlantic Treaty Organization Advisory Group for Aerospace Research and Development, AGARD-AG-207.

Takahashi, T. T. (1997), "Measurement of Air Flow Characteristics using Seven Hole Cone Probes." Aerospace Sciences Meeting \& Exhibit, Reno, NV, AIAA.

Taremi, f. (2005), "Endwall Flows in Transonic Linear Turbine Cascades," Ph.D. Thesis Proposal, Department of Mechanical \& Aerospace Engineering, Carleton University, Ottawa, Canada.

Tavoularis, S. (2005), Measurement in Fluid Mechanics. New York, Cambridge University Press.

Tavoularis, S., and Szymczak, M. (1989), "Displacement Effect of Square-Ended Pitot Tubes in Shear Flows," Experiments in Fluids, Vol. 7, pp. 33-37.

Taylor, B. N., and Kuyatt, C. E. (1994), "Guidelines for Evaluating and Expressing the Uncertainty of NIST Measurement Results," NIST-TN-1297.

Walchner, O. (1939), "The Effect of Compressibility on the Pressure Reading of a Prandtl Pitot Tube at Subsonic Flow Velocity," NACA Technical Memorandum 917.

Walsche, D. E., and Garner, H. C. (1960), "Usefulness of Various Pressure Probes in Fluctuating Low Speed Flows," Brit. ARC 21714.

Wenger, C. W., and Devenport, W. J. (1999), "Seven-Hole Pressure Probe Calibration Method utilizing Look-Up Error Tables," AIAA Journal, Vol. 37, pp. 675-679.

Yaras, M. I. (1990), "Measurement and Prediction of Tip-Clearance Effects in a Linear Turbine Cascade," Ph.D. Thesis, Department of Mechanical \& Aerospace Engineering, Carleton University, Ottawa, Canada. 
Young, A. D., and Mass, J. N. (1936), "The Behavior of a Pitot Tube in a Transverse Total Pressure Gradient," ARC R\&M 1770.

Zeiger, M. D., Chalmeta, L. P., and Telionis, D. P. (1998), "Tip Geometry Effects on Calibration and Performance of Seven-Hole Probes," AIAA 98-2810.

Zilliac, G. G. (1993), "Modelling, Calibration, and Error Analysis of Seven-Hole Pressure Probes," Experiments in Fluids, Vol. 14, pp. 104-120. 


\section{Appendix A:}

\section{Pressure Transducer Calibration}

The pressure transducers used here were calibrated with the water micromanometer constructed by Benner (2003) and shown in Figure A.1. This micromanometer has a fullscale range of $0-30 \mathrm{~cm}$ of water and was filled with fresh distilled water prior to use to reduce water density uncertainty. Large diameter water columns were used to reduce capillary attraction for improved accuracy; the micromanometer also stands on screwtype feet to allow for levelling prior to use. Benner states that the micromanometer positional tolerances are $\pm 0.025 \mathrm{~mm}_{2} \mathrm{O}$, but frequent use has bent the needle on the vacuum side of the apparatus. The present author, through repeated use of the setup, estimates positional tolerances of about $\pm 0.05 \mathrm{~mm} \mathrm{H}_{2} \mathrm{O}$.

Using this micromanometer, curve fits for applied pressure versus pressure transducer output voltage were generated. This relation was described by:

$$
P=C_{1} V^{3}+C_{2} V^{2}+C_{3} V+C_{4}
$$


where $P$ is pressure, $V$ is the output voltage, and $C_{1}$ through $C_{4}$ are calibration coefficients. $C_{1}$ through $C_{3}$ are found through calibration; $C_{4}$ is measured before and after transducer use to account for any shift in the zero offset of the transducers. Table A.1 shows typical calibration coefficients for the eight transducers used here. Figure A.2 provides sample calibration curves for three of these transducers.

\begin{tabular}{|c|c|c|c|c|}
\hline Transducer & $C_{1}$ & $C_{2}$ & $C_{3}$ & $C_{4}$ \\
\hline 1 & 0.077 & -0.805 & 493.0 & -1701.1 \\
\hline 2 & 0.085 & -0.930 & 497.1 & -1737.4 \\
\hline 3 & 0.080 & -0.885 & 488.2 & -1703.5 \\
\hline 4 & 0.839 & -9.01 & 519.9 & -1746.4 \\
\hline 5 & 0.117 & -1.29 & 495.7 & -1705.6 \\
\hline 6 & 0.119 & -1.30 & 492.2 & -1710.7 \\
\hline 7 & 0.507 & -5.65 & 507.7 & -1728.2 \\
\hline 8 & 0.439 & -4.81 & 503.4 & -1718.8 \\
\hline
\end{tabular}

Table A.1: Sample Transducer Calibration Coefficients

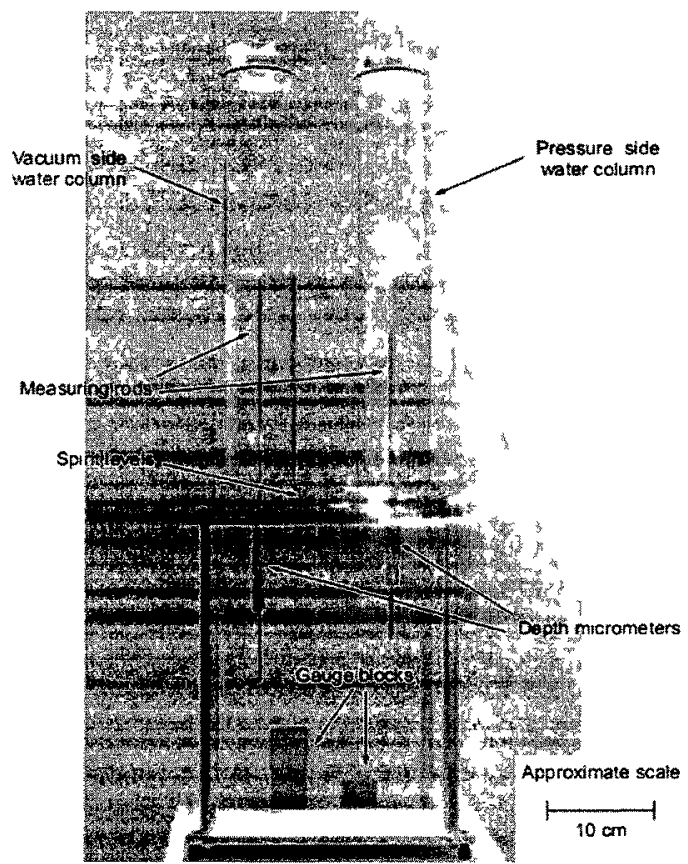

Figure A.1: Water Micromanometer, from Benner (2003) 


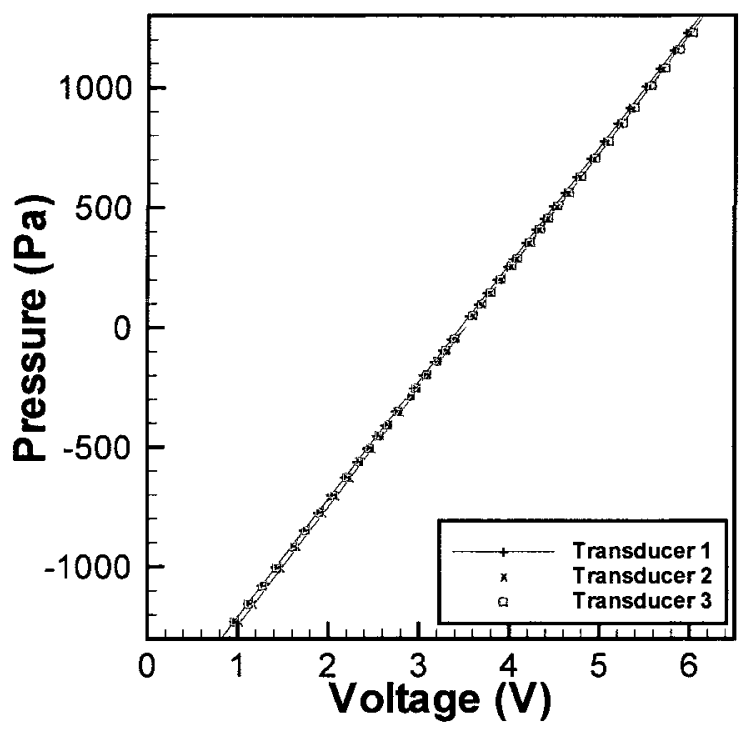

Figure A.2: Sample Transducer Calibration Curve 


\section{Appendix B:}

\section{Supplemental Results from the Kiel Probe}

This appendix includes results from the Kiel probe that were omitted from the main text for brevity. These results, as referenced in Section 4.3, show the same trends as previously discussed.

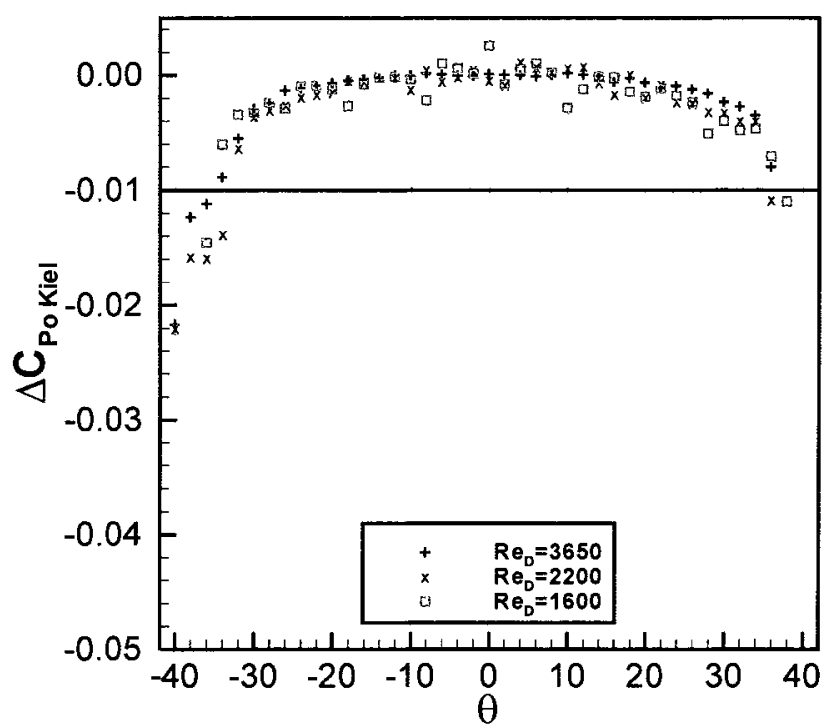

Figure B.1: Kiel Probe Results for Total Pressure at Various Reynolds Numbers and

$$
\mathrm{Tu}=\mathbf{1 . 5 \%}
$$




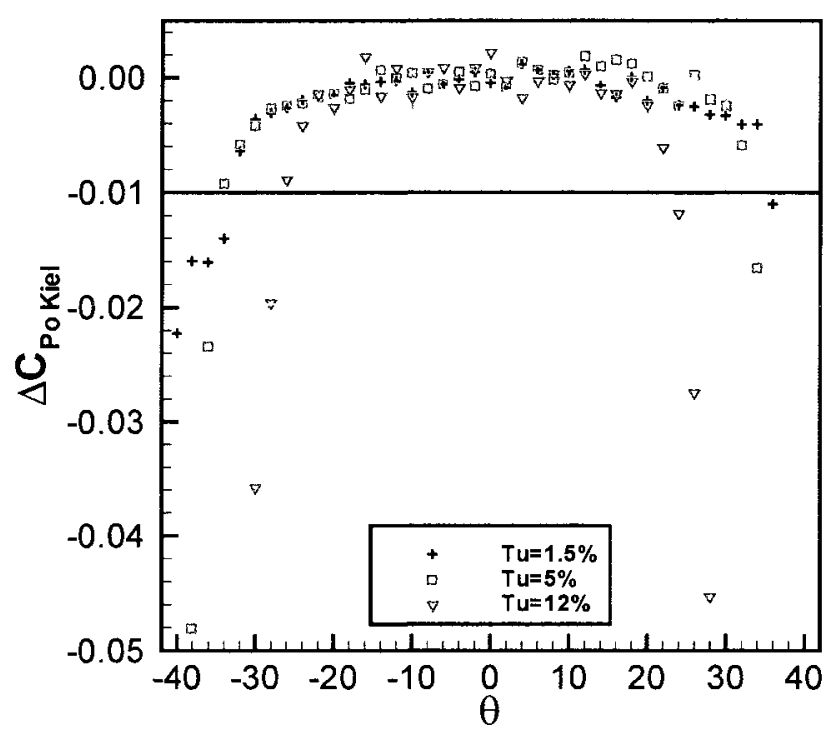

Figure B.2: Kiel Probe Results for Total Pressure at $\mathrm{Re}_{\mathrm{D}}=\mathbf{2 2 0 0}$ and Various Turbulence Intensities
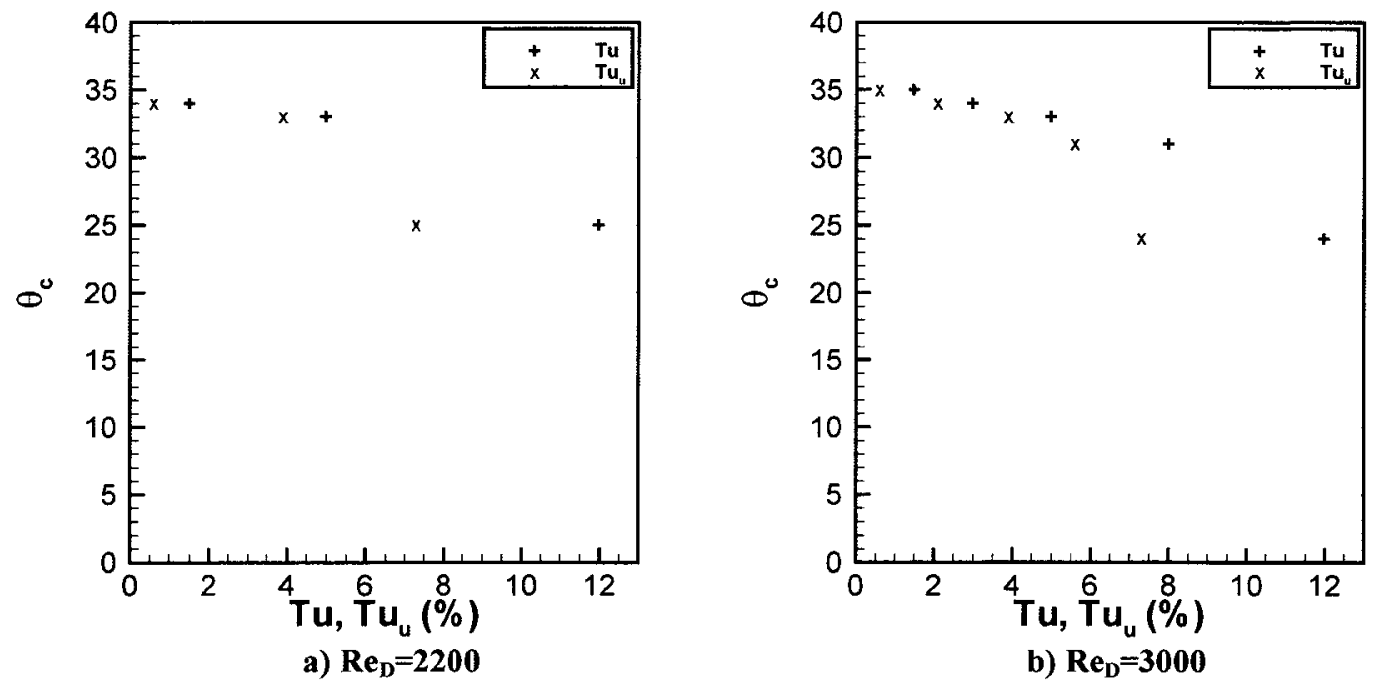

Figure B.3: Kiel Probe Results for Critical Angle as a function of Turbulence Intensity 


\section{Appendix C:}

\section{Supplemental Results from the Three-Hole}

\section{Pressure Probe}

This appendix provides all the results collected using the three-hole pressure probe. These results include those discussed in Section 4.4 as well as those omitted from the main text for brevity. Figures C.1 through C.10 show the effects of turbulence intensity at constant Reynolds number; Figures C.11 and C.20 show the effects of Reynolds number at constant turbulence intensity. Figures C.21 and C.22 show a sample of the combined effects of both flow parameters. 


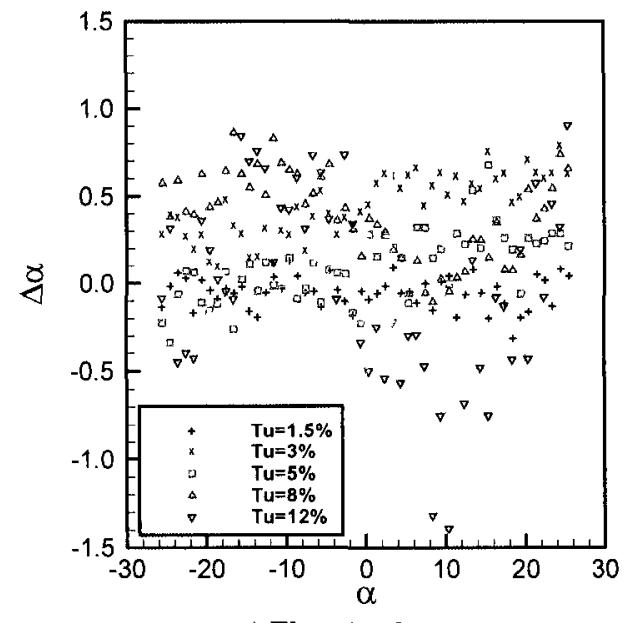

a) Flow Angle

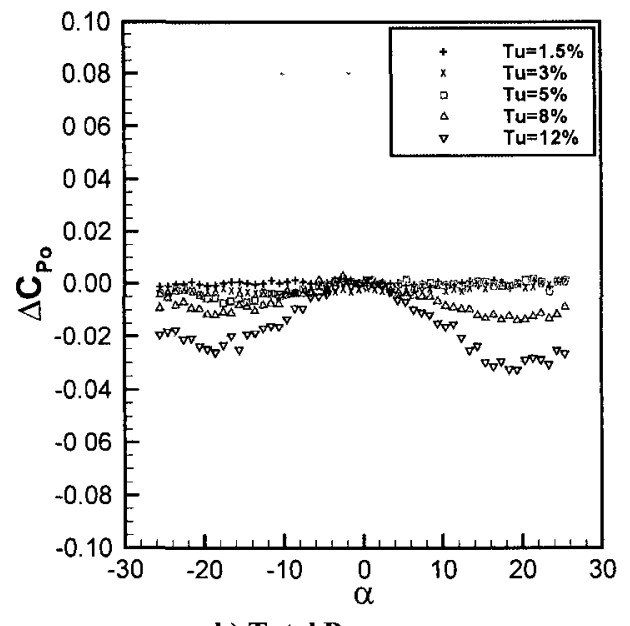

b) Total Pressure

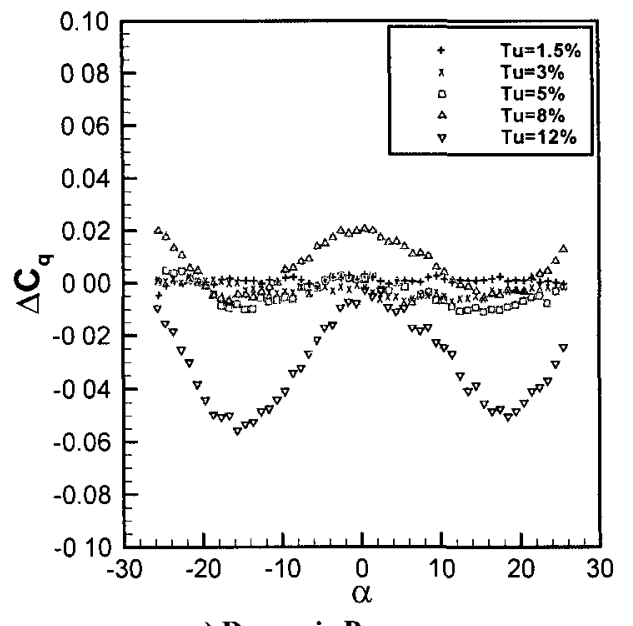

c) Dynamic Pressure

Figure C.1: Three-Hole Pressure Probe Results: Calibration at $\operatorname{Re}_{\mathrm{D}}=1850, T u=1.5 \%$; $\mathrm{Data}_{\mathrm{at}} \operatorname{Re}_{\mathrm{D}}=1850, \mathrm{Tu}$ as shown
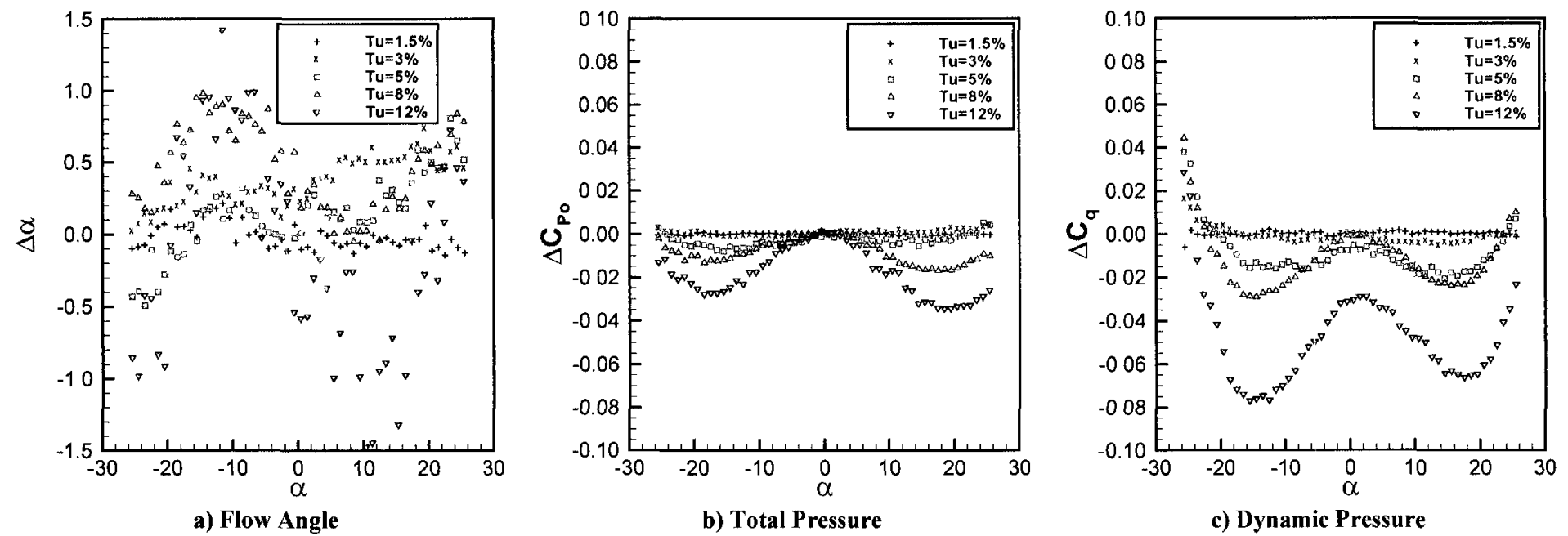

Figure C.2: Three-Hole Pressure Probe Results: Calibration at $\operatorname{Re}_{\mathrm{D}}=2500, T u=1.5 \%$; Data at $\operatorname{Re}_{\mathrm{D}}=2500$, $T u$ as shown 

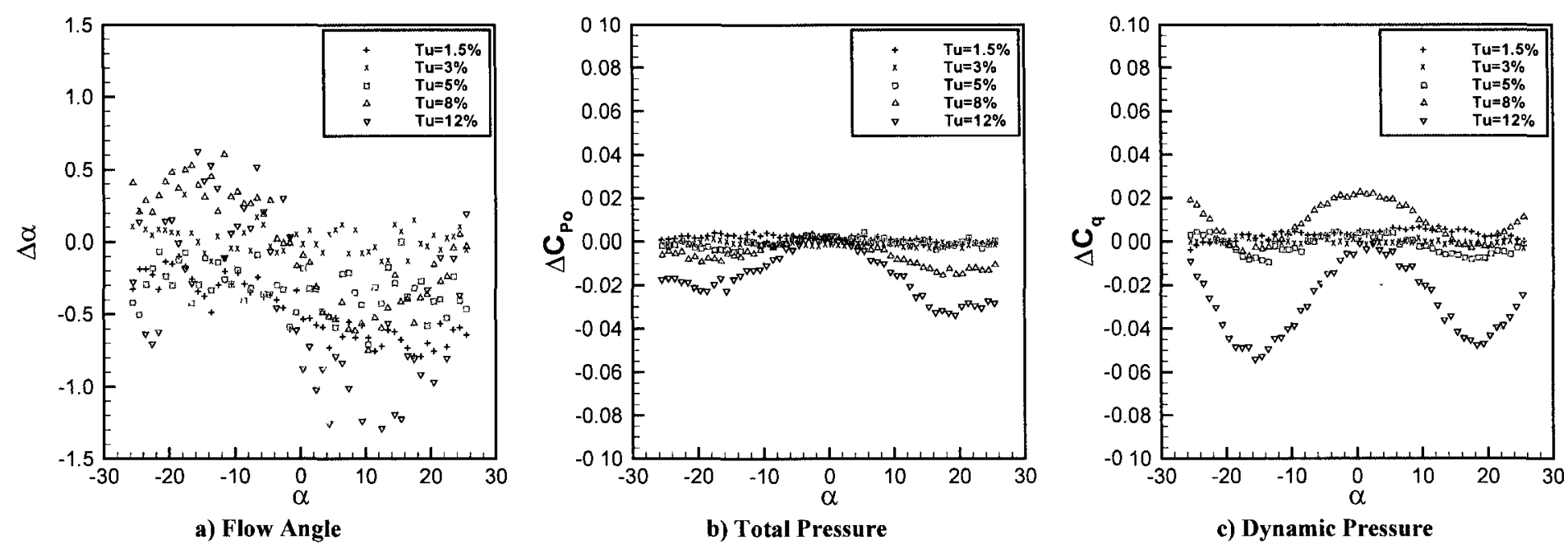

Figure C.4: Three-Hole Pressure Probe Results: Calibration at $R_{\mathrm{D}}=2500, \mathrm{Tu}=3 \%$; Data at $\operatorname{Re}_{\mathrm{D}}=2500$, $\mathrm{Tu}$ as shown 


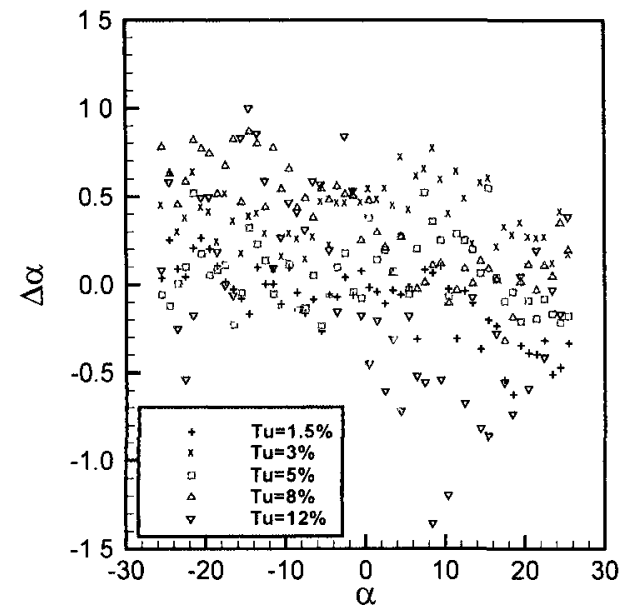

a) Flow Angle

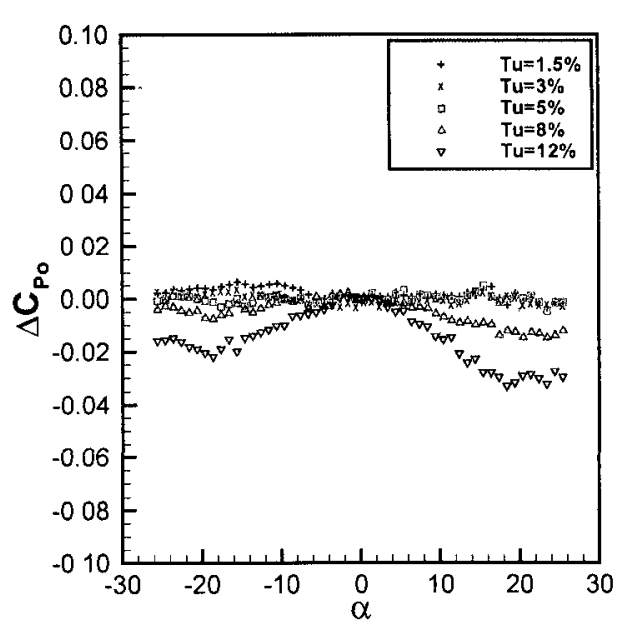

b) Total Pressure

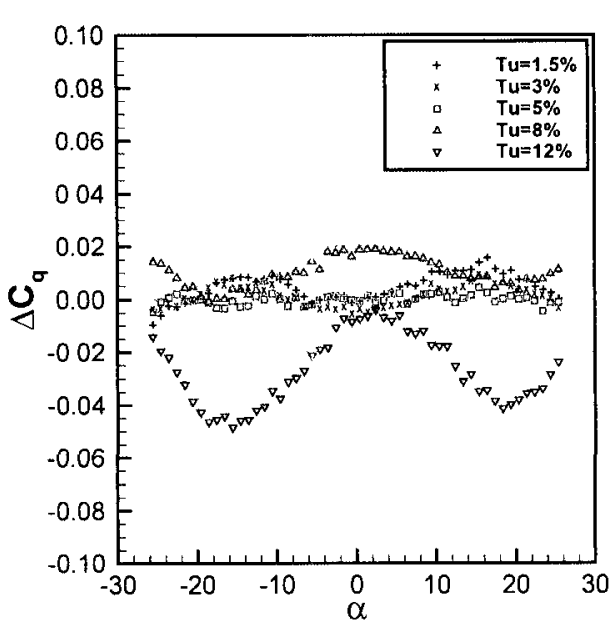

c) Dynamic Pressure

Figure C.5: Three-Hole Pressure Probe Results: Calibration at $\operatorname{Re}_{\mathrm{D}}=1850, T u=5 \%$; $\mathrm{Data}_{\mathrm{at}} \operatorname{Re}_{\mathrm{D}}=1850$, $\mathrm{Tu}$ as shown
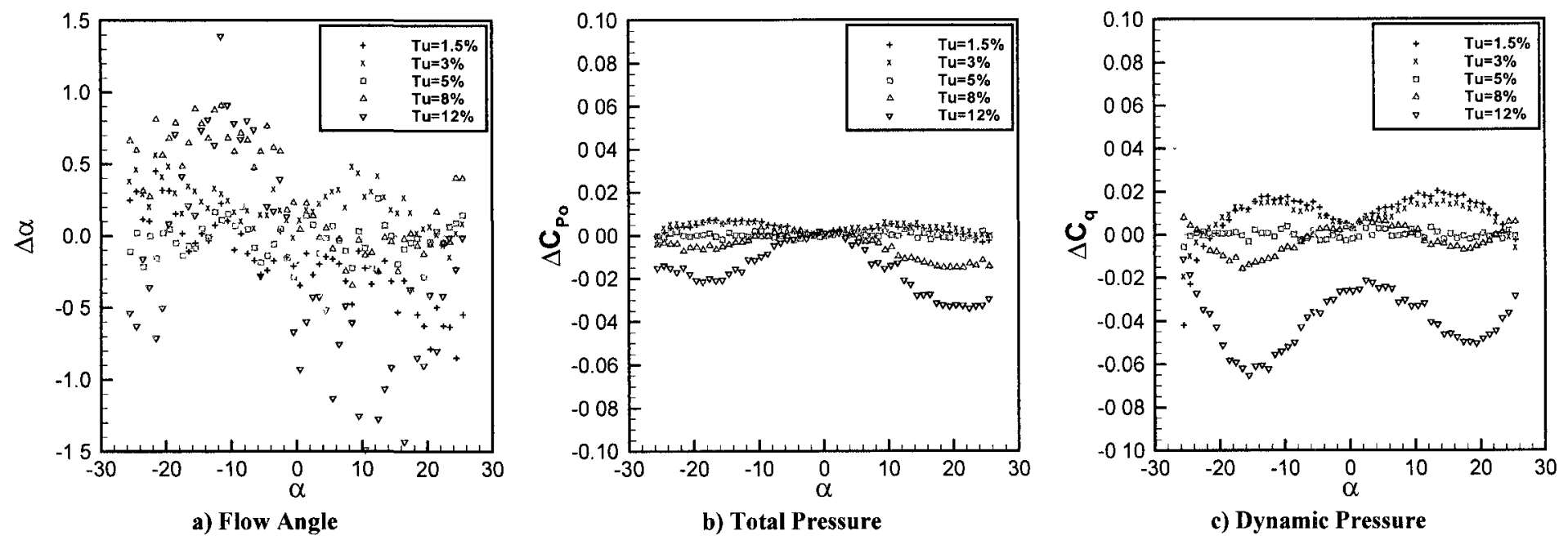

Figure C.6: Three-Hole Pressure Probe Results: Calibration at $\operatorname{Re}_{D}=2500, T u=5 \%$; $D a t a$ at $\operatorname{Re}_{D}=2500, T_{u}$ as $\operatorname{shown}$ 

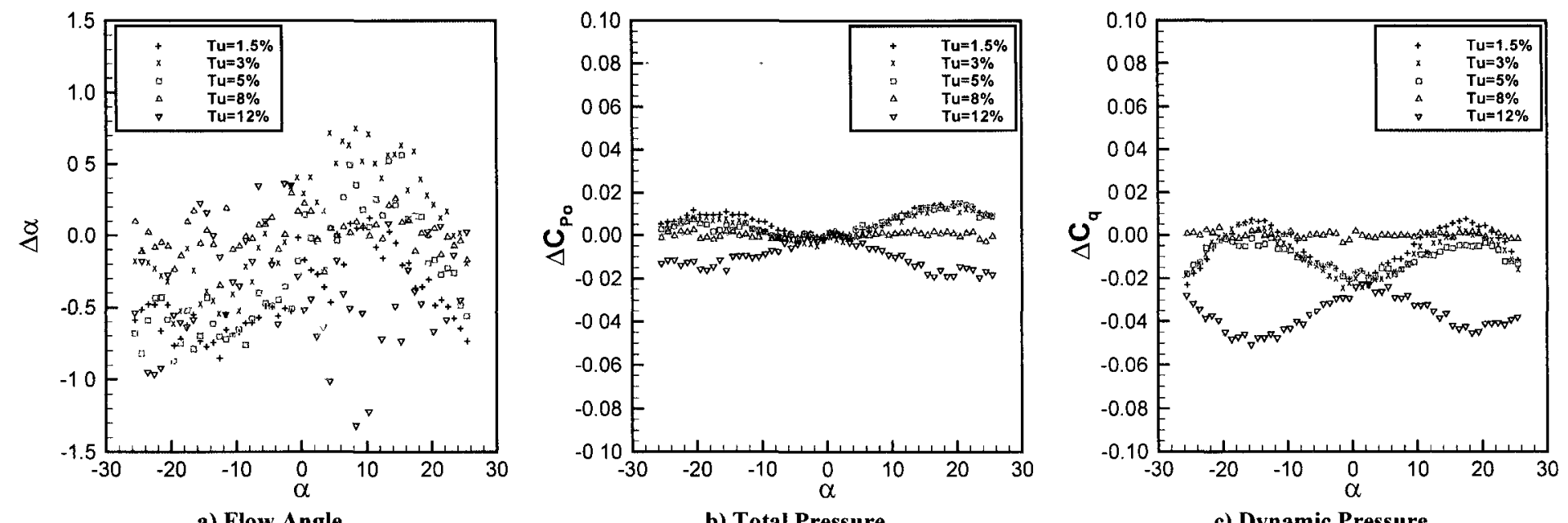

Figure C.8: Three-Hole Pressure Probe Results: Calibration at $R_{D}=2500, T u=8 \%$; Data at $R_{D}=2500$, $T u$ as shown

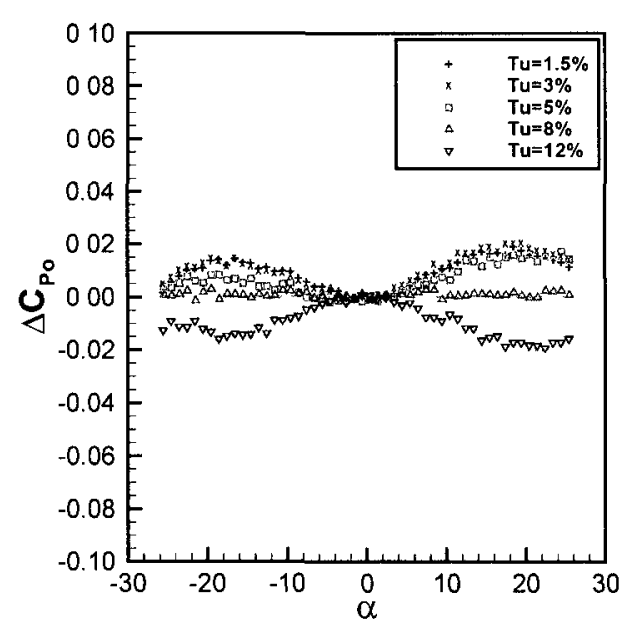

b) Total Pressure

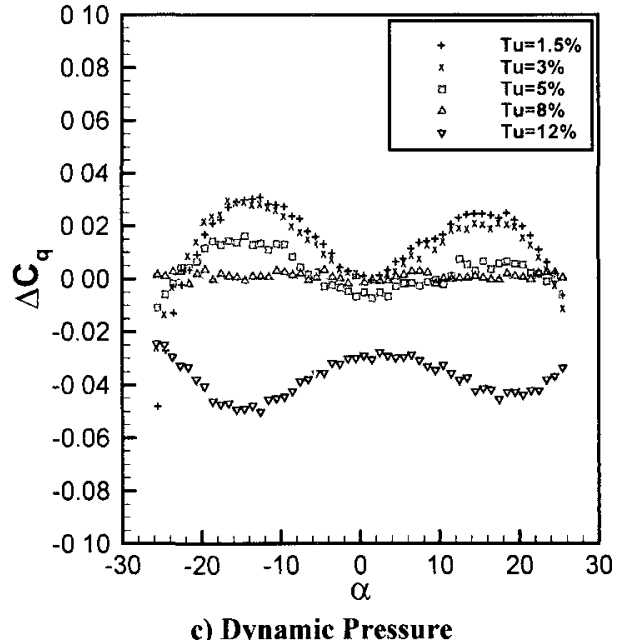

c) Dynamic Pressure 

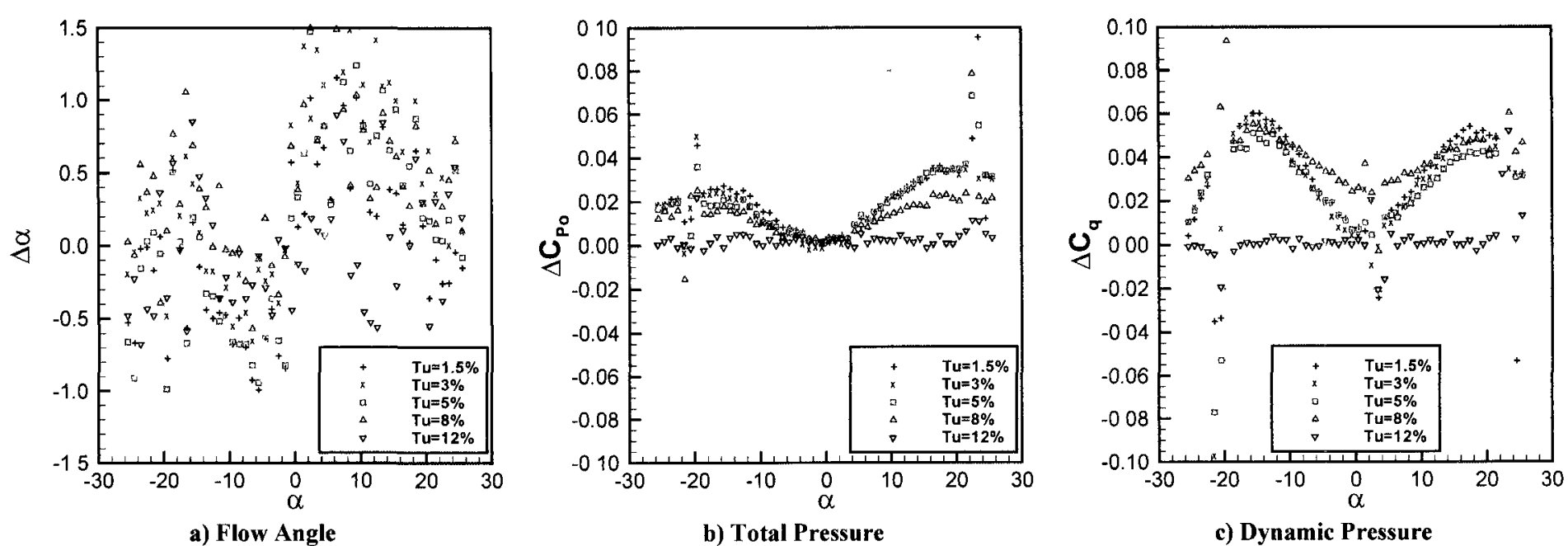

Figure C.10: Three-Hole Pressure Probe Results: Calibration at $\operatorname{Re}_{\mathrm{D}}=\mathbf{2 5 0 0}, \mathrm{Tu}=12 \%$; Data at $\mathrm{Re}_{\mathrm{D}}=\mathbf{2 5 0 0}$, $\mathrm{Tu}$ as shown 

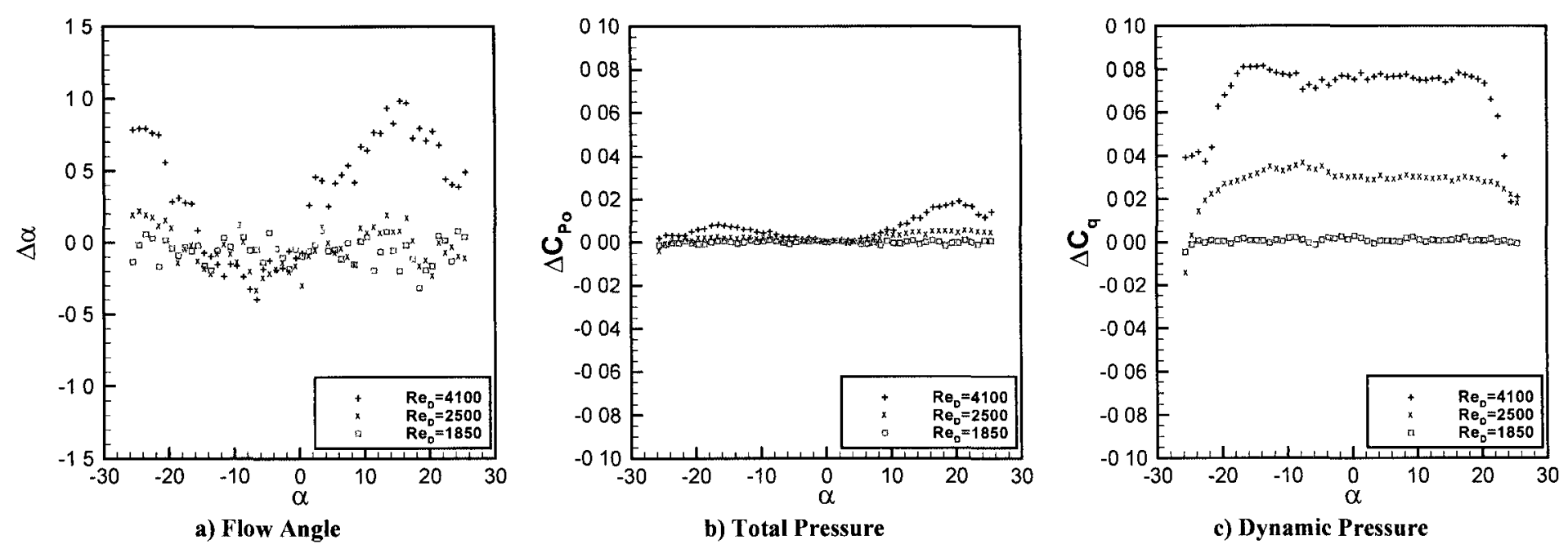

Figure C.12: Three-Hole Pressure Probe Results: Calibration at $\operatorname{ReD}=4100, T u=1.5 \%$; Data at $\operatorname{ReD}$ as shown, $T u=1.5 \%$ 

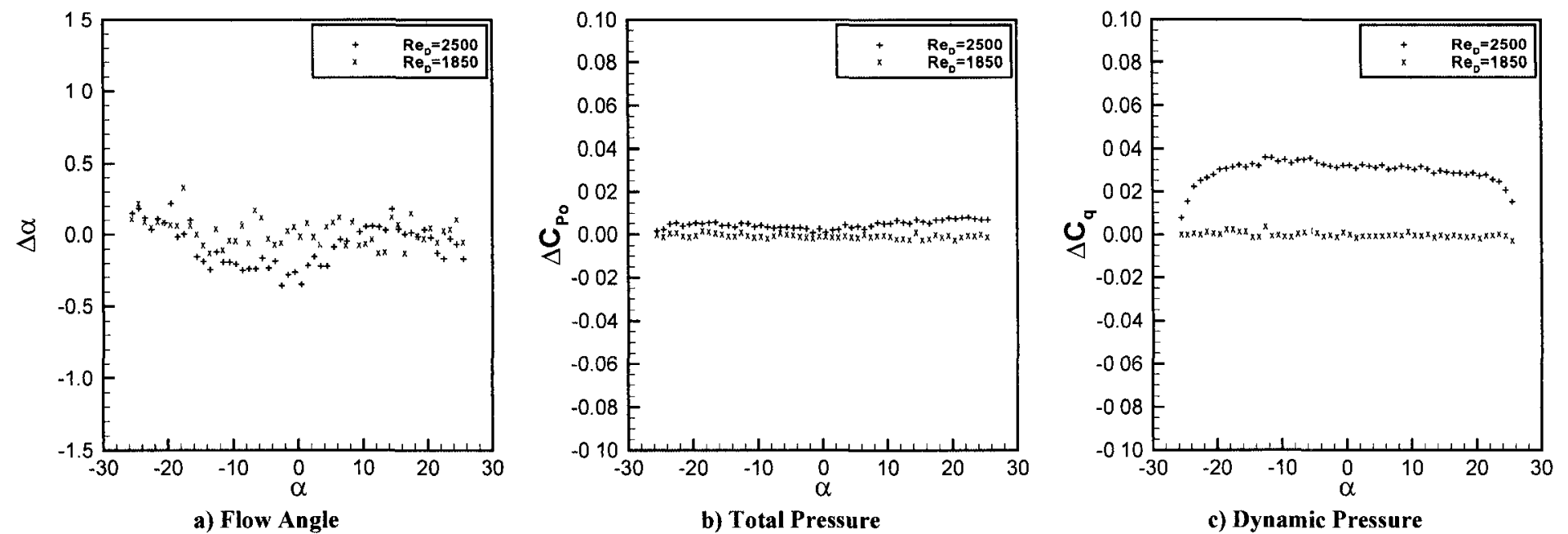

Figure C.14: Three-Hole Pressure Probe Results: Calibration at $\operatorname{Re}_{\mathrm{D}}=\mathbf{2 5 0 0}, \mathrm{Tu}=3 \%$; Data at $\mathrm{Re}_{\mathrm{D}}$ as shown, $\mathrm{Tu}=3 \%$ 

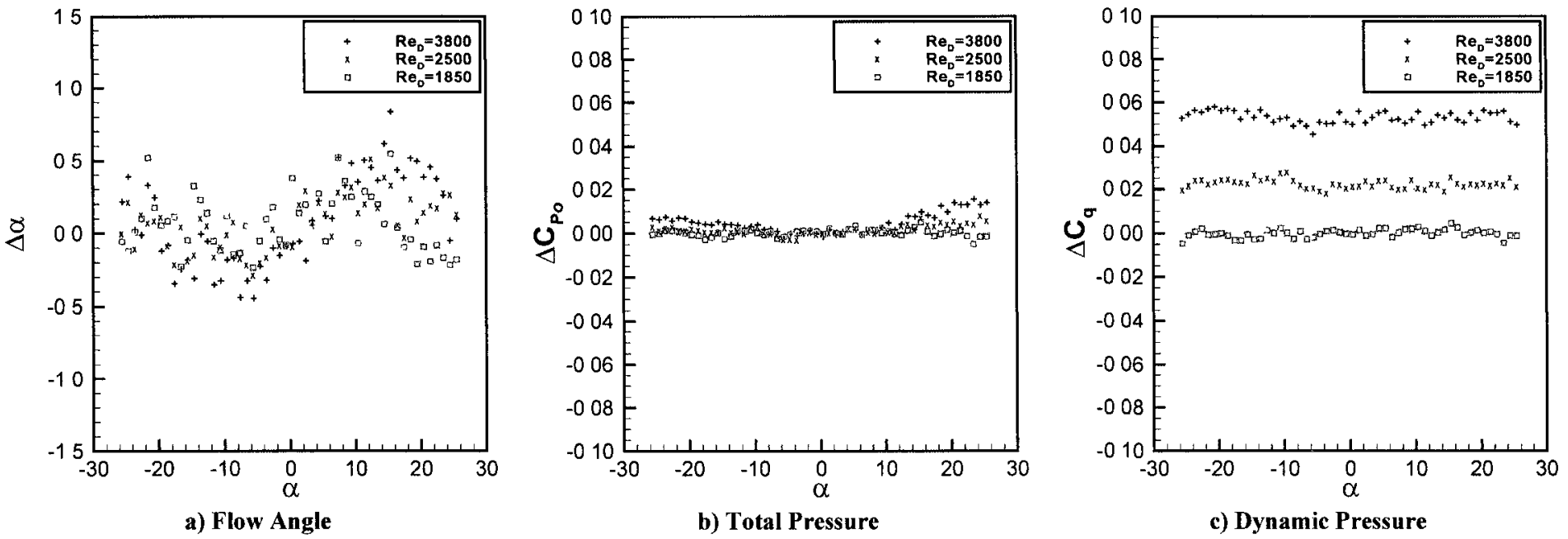

Figure C.16: Three-Hole Pressure Probe Results: Calibration at $\operatorname{Re}_{\mathrm{D}}=3800, \mathrm{Tu}=5 \%$; Data at $\operatorname{Re}_{\mathrm{D}}$ as shown, $\mathrm{Tu}=5 \%$ 

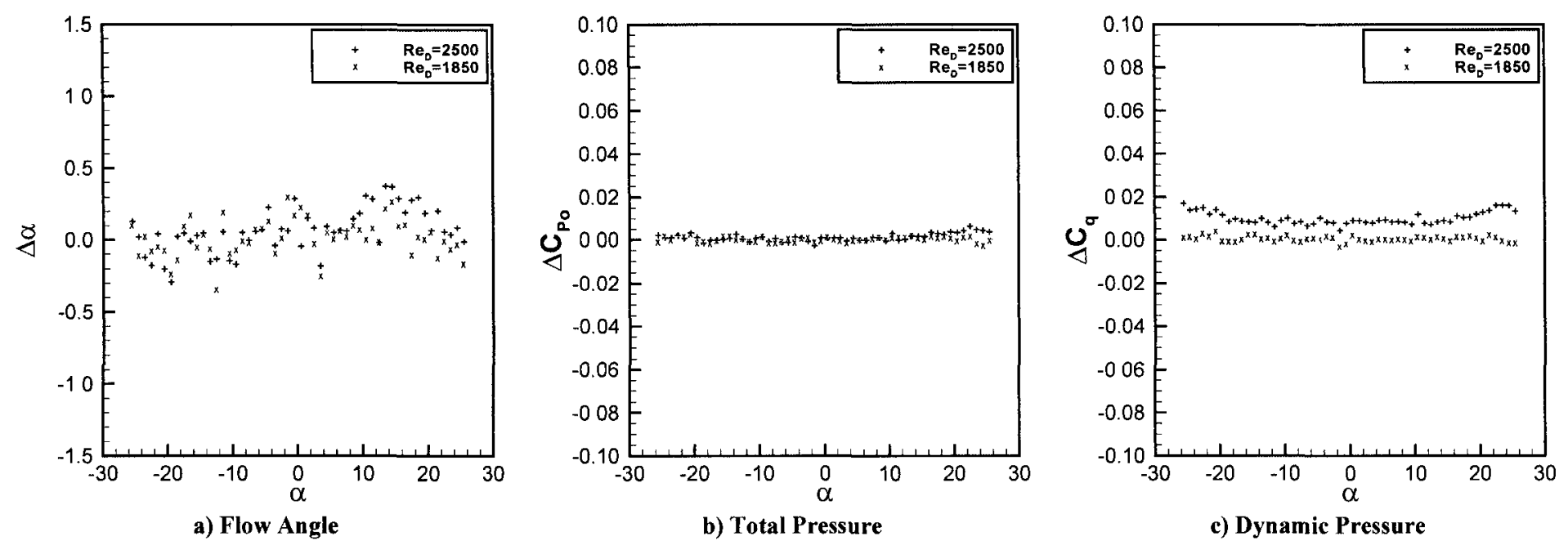

Figure C.18: Three-Hole Pressure Probe Results: Calibration at $\operatorname{Re}_{\mathrm{D}}=\mathbf{2 5 0 0}, \mathrm{Tu}=\mathbf{8 \%}$; Data at $\operatorname{Re}_{\mathrm{D}}$ as shown, $\mathrm{Tu}=\mathbf{8 \%}$ 

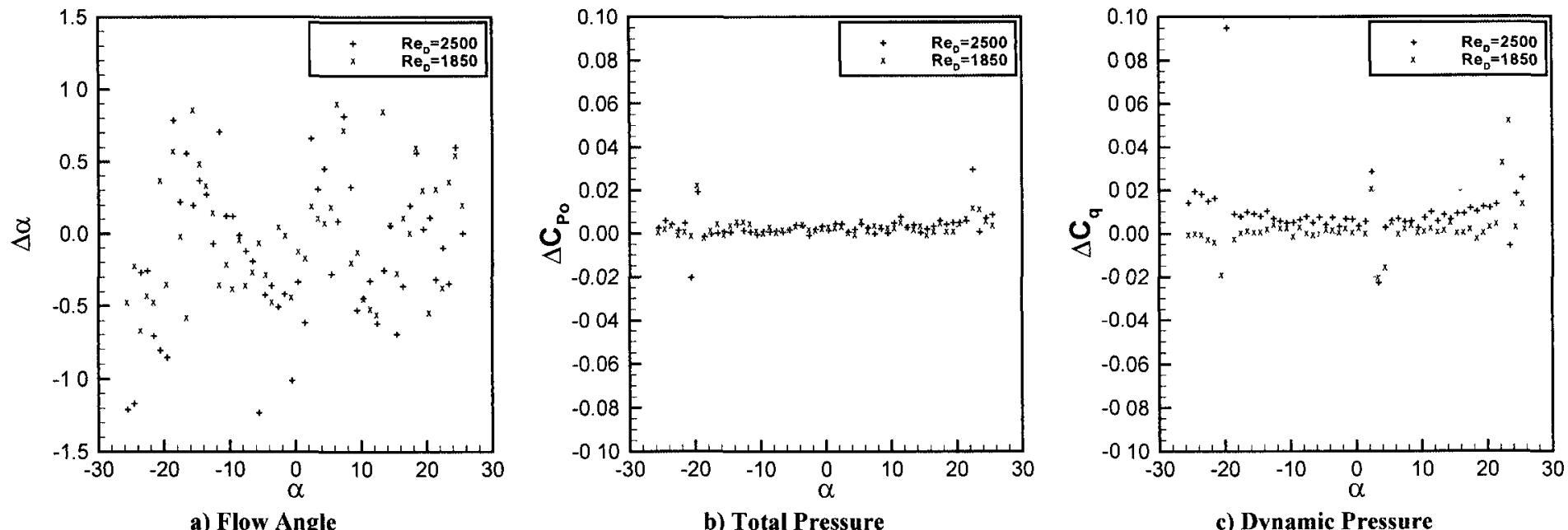

Figure C.20: Three-Hole Pressure Probe Results: Calibration at $\operatorname{Re}_{\mathrm{D}}=\mathbf{2 5 0 0}, \mathrm{Tu}=12 \%$; Data at $\operatorname{Re}_{\mathrm{D}}$ as shown, $\mathrm{Tu}=12 \%$ 

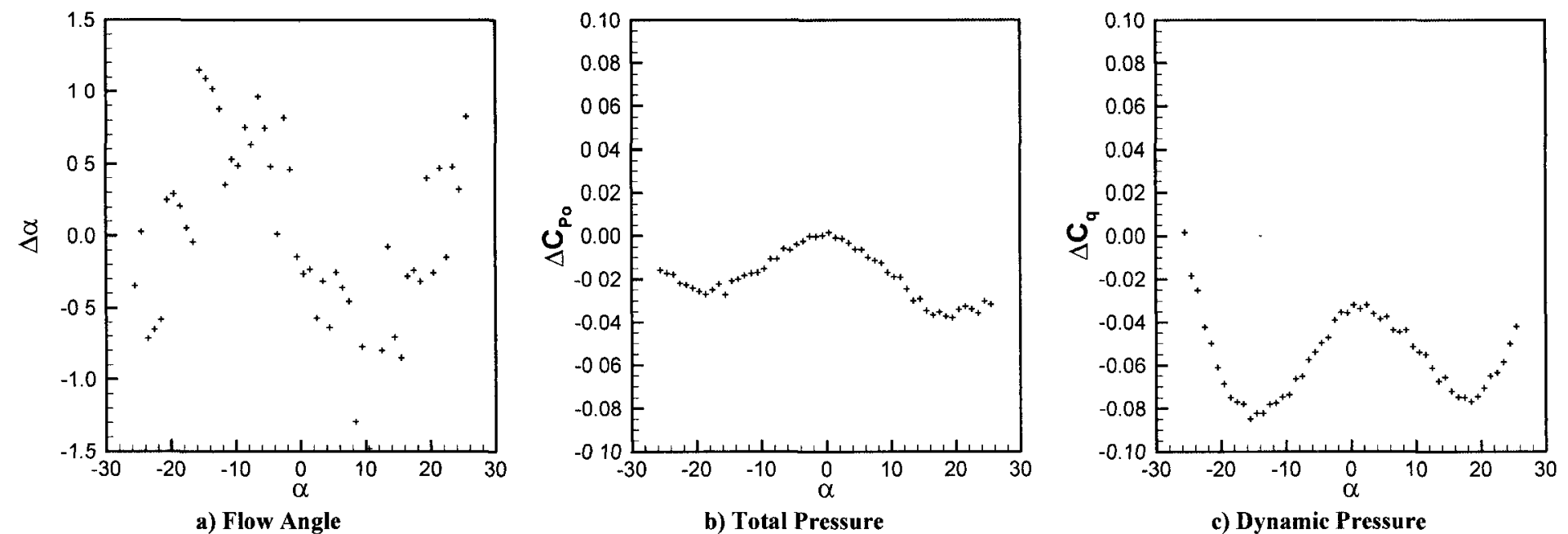

Figure C.22: Three-Hole Pressure Probe Results: Calibration at $\operatorname{Re}_{\mathrm{D}}=1850, \mathrm{Tu}=12 \%$; Data at $\operatorname{Re}_{\mathrm{D}}=2500, \mathrm{Tu}=1.5 \%$ 


\section{Appendix D:}

\section{Supplemental Results from the Seven-Hole}

\section{Pressure Probe}

This appendix provides all the results collected in the present study using both sevenhole pressure probes. These results include those discussed in Section 4.5 as well as those omitted from the main text for brevity. Figures D.1 through D.12 show the effects of turbulence intensity at constant Reynolds number on probe 7HP1; Figures D.13 and D.29 show the effects of Reynolds number at constant turbulence intensity on probe 7HP1. Figures D.30 through D.39 show similar results for the effects of each parameter on probe $7 \mathrm{HP} 2$. 

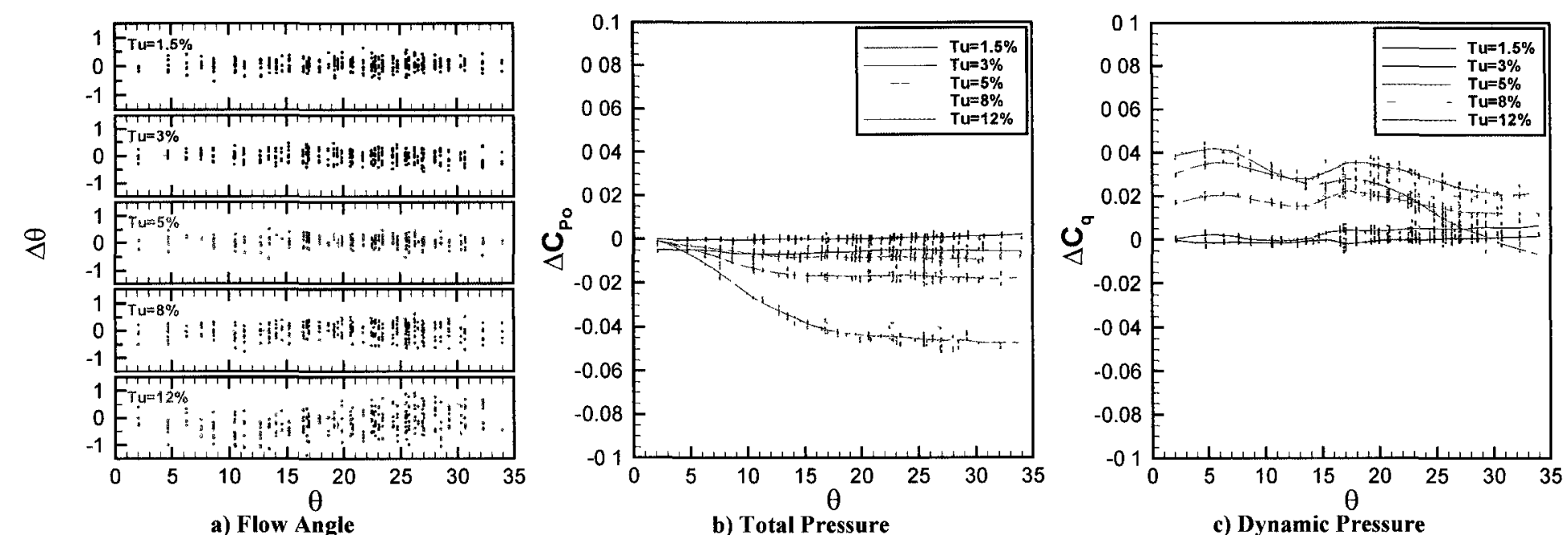

Figure D.1: Seven-Hole Pressure Probe Results: Calibration at $R_{D}=2500, T u=1.5 \%$; Data at $R_{D}=2500$, $T u$ as shown
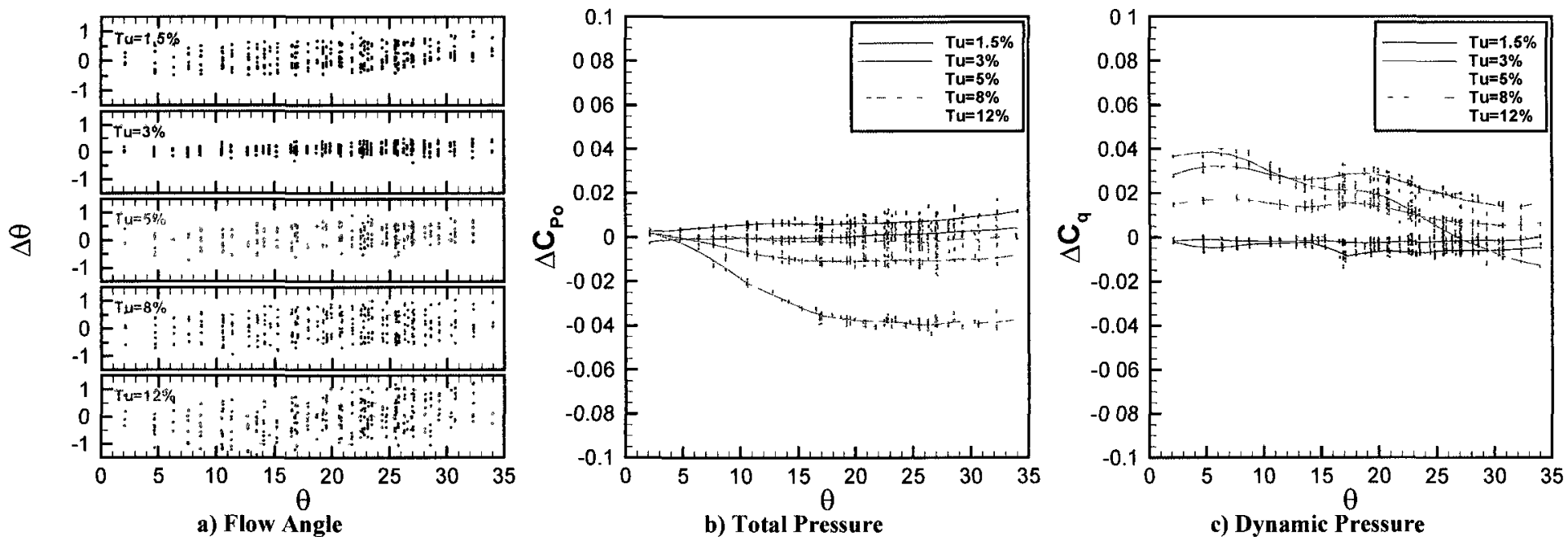

Figure D.2: Seven-Hole Pressure Probe Results: Calibration at $\operatorname{Re}_{D}=2500, T u=3 \%$; Data at $\operatorname{Re}_{D}=2500$, $T u$ as shown 


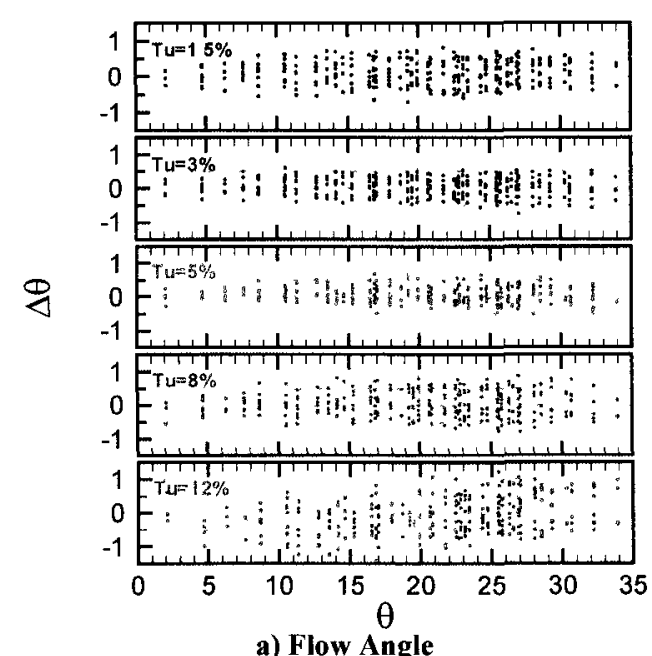

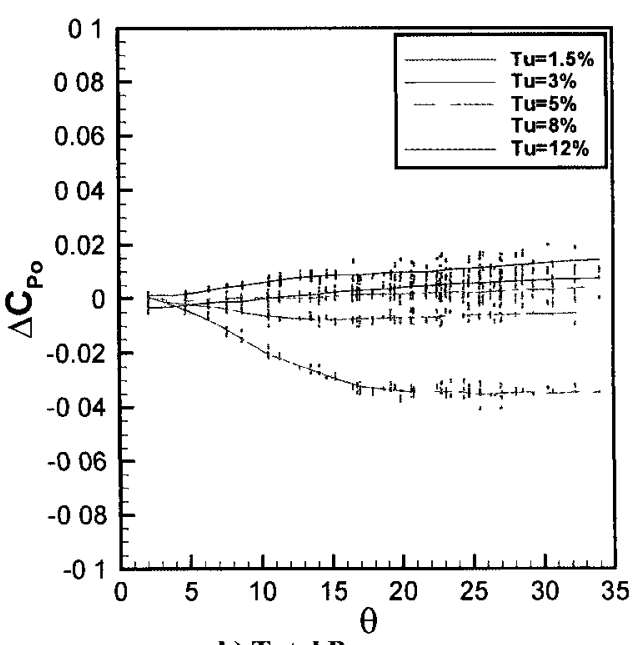

b) Total Pressure

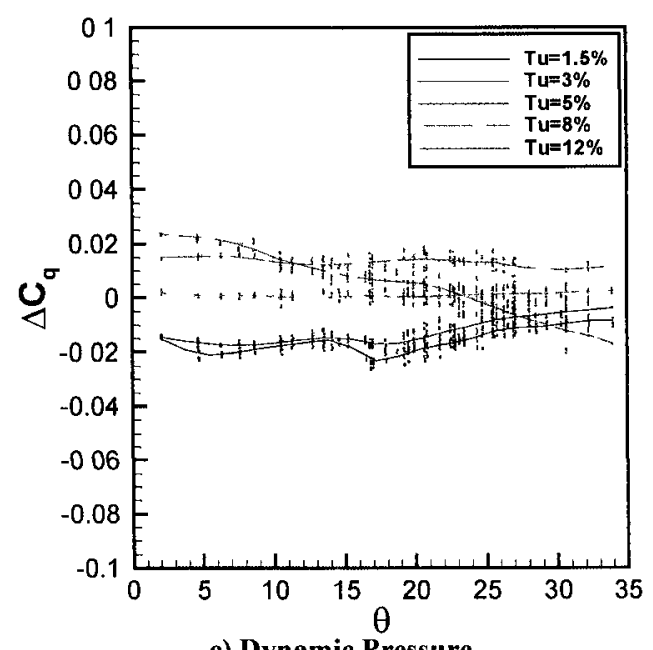

c) Dynamic Pressure

Figure D.3: Seven-Hole Pressure Probe (7HP1) Results: Calibration at $R_{D}=2500, T u=5 \%$; Data at $R_{D}=2500$, Tu as shown
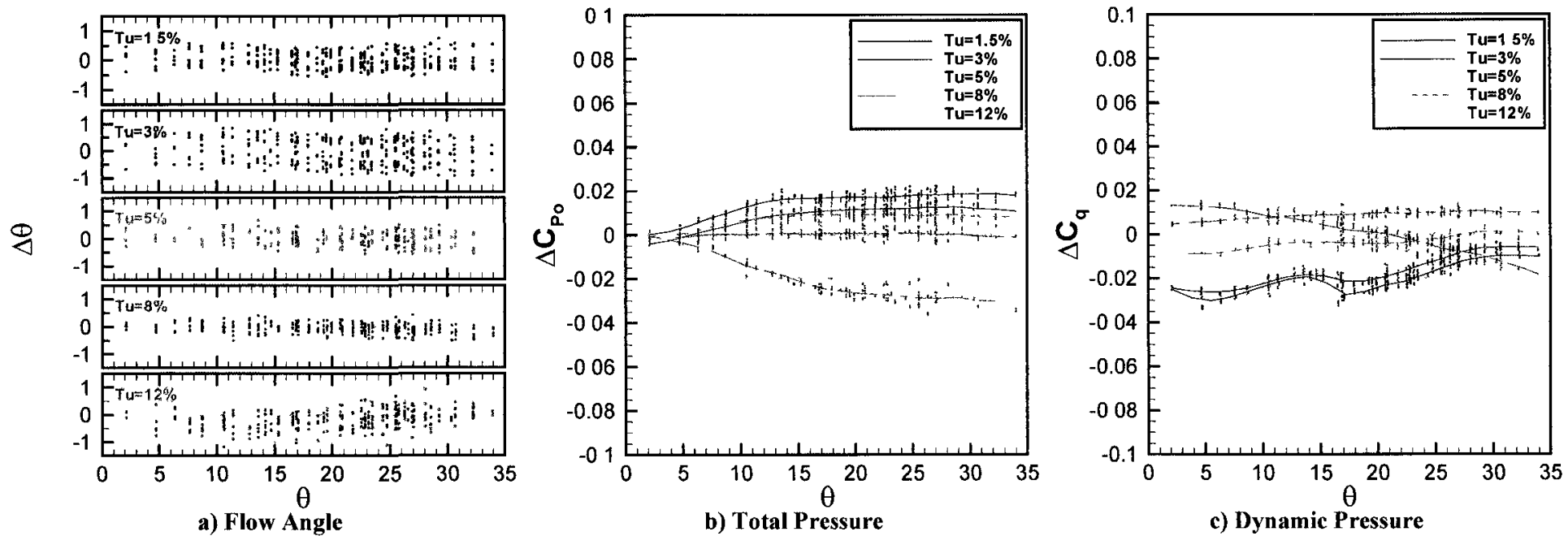

Figure D.4: Seven-Hole Pressure Probe (7HP1) Results: Calibration at $\operatorname{Re}_{\mathrm{D}}=2500, \mathrm{Tu}=8 \%$; Data at $\mathrm{Re}_{\mathrm{D}}=2500$, Tu as shown 

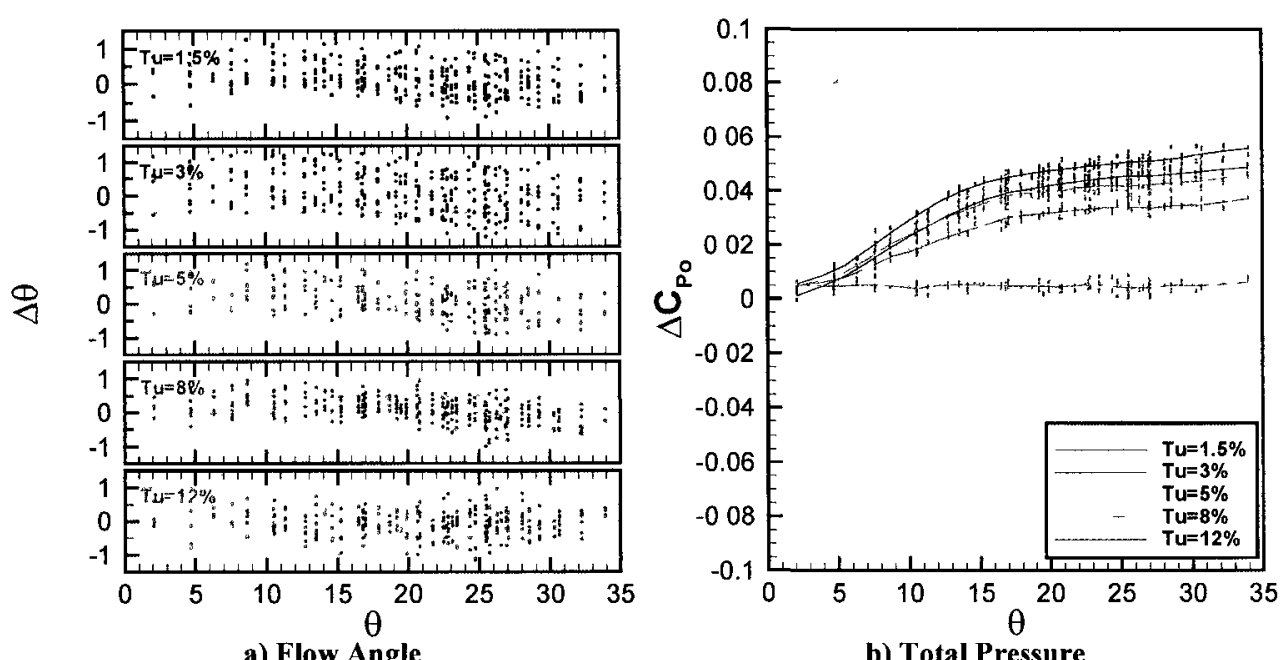

a) Flow Angle

b) Total Pressure

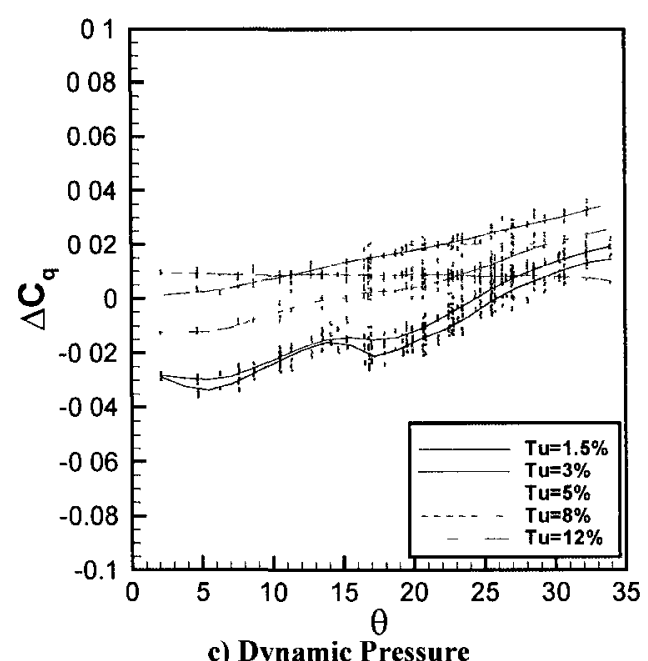

Figure D.5: Seven-Hole Pressure Probe (7HP1) Results: Calibration at $\operatorname{Re}_{\mathrm{D}}=2500$, $\mathrm{Tu}=12 \%$; Data at $\operatorname{Re}_{\mathrm{D}}=2500$, $\mathrm{Tu}$ as shown
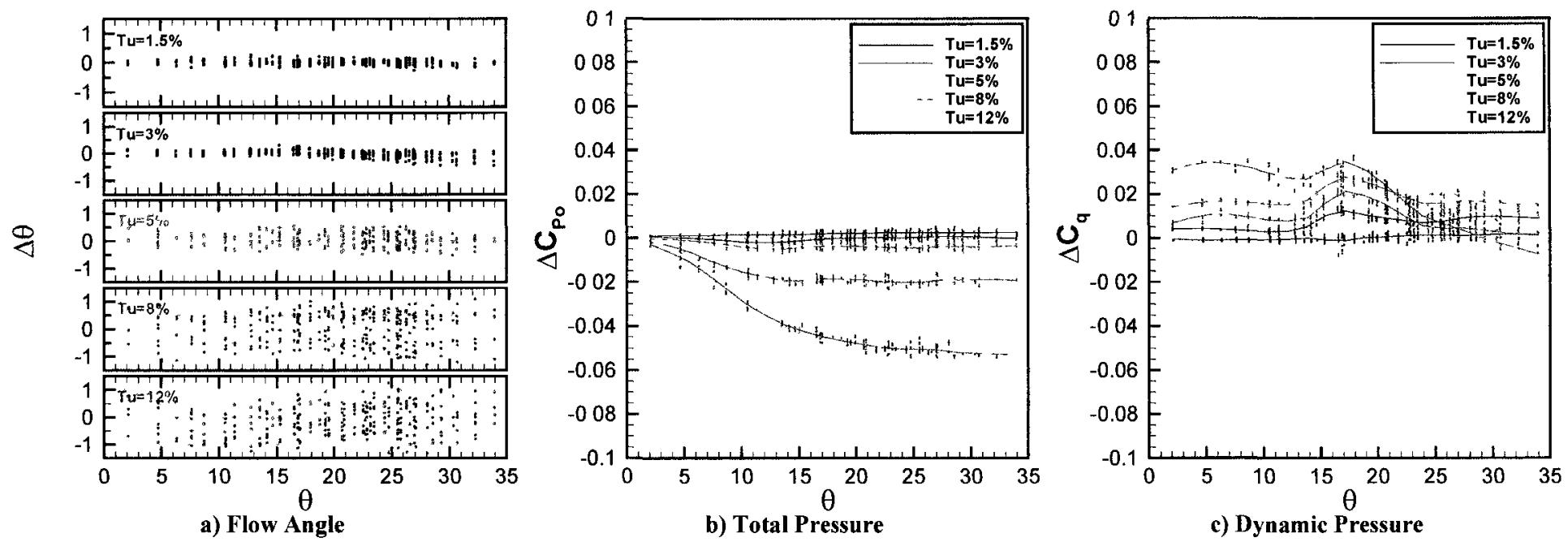

Figure D.6: Seven-Hole Pressure Probe (7HP1) Results: Calibration at $\operatorname{Re}_{D}=3900, T u=1.5 \%$; Data at $\operatorname{Re}_{D}=3900$, $T u$ as shown 

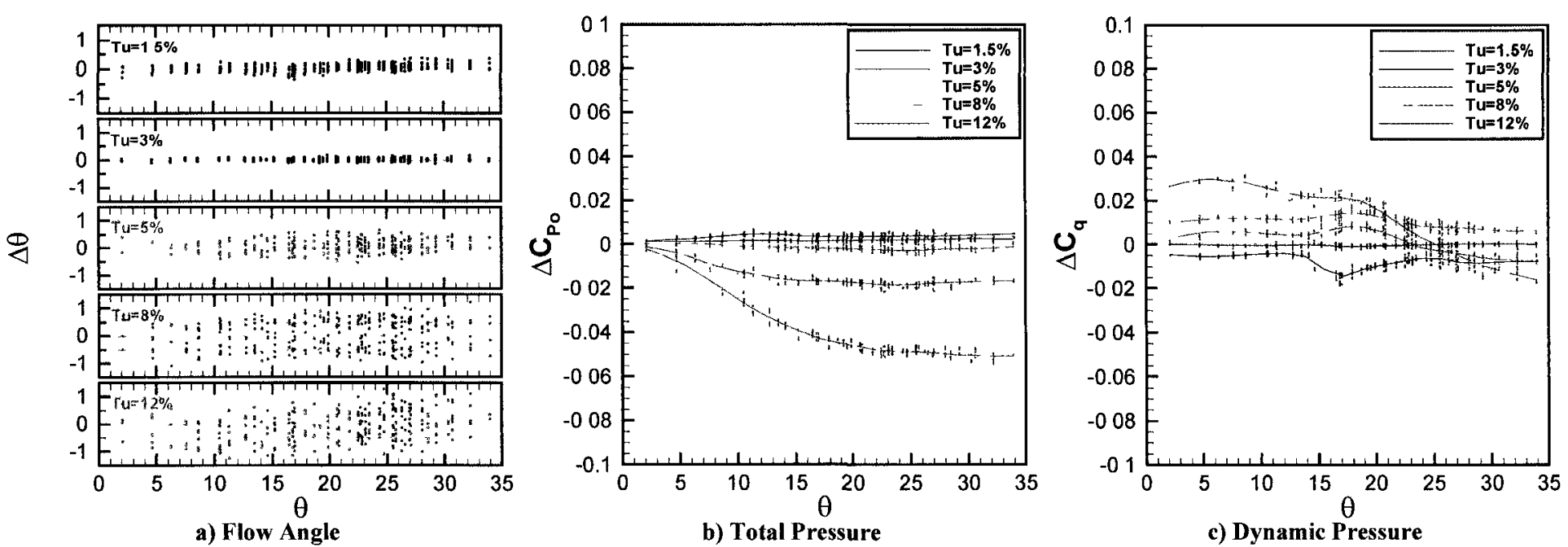

Figure D.7: Seven-Hole Pressure Probe (7HP1) Results: Calibration at $R_{D}=3900, T u=3 \%$; Data at $R_{D}=3900$, $T u$ as shown
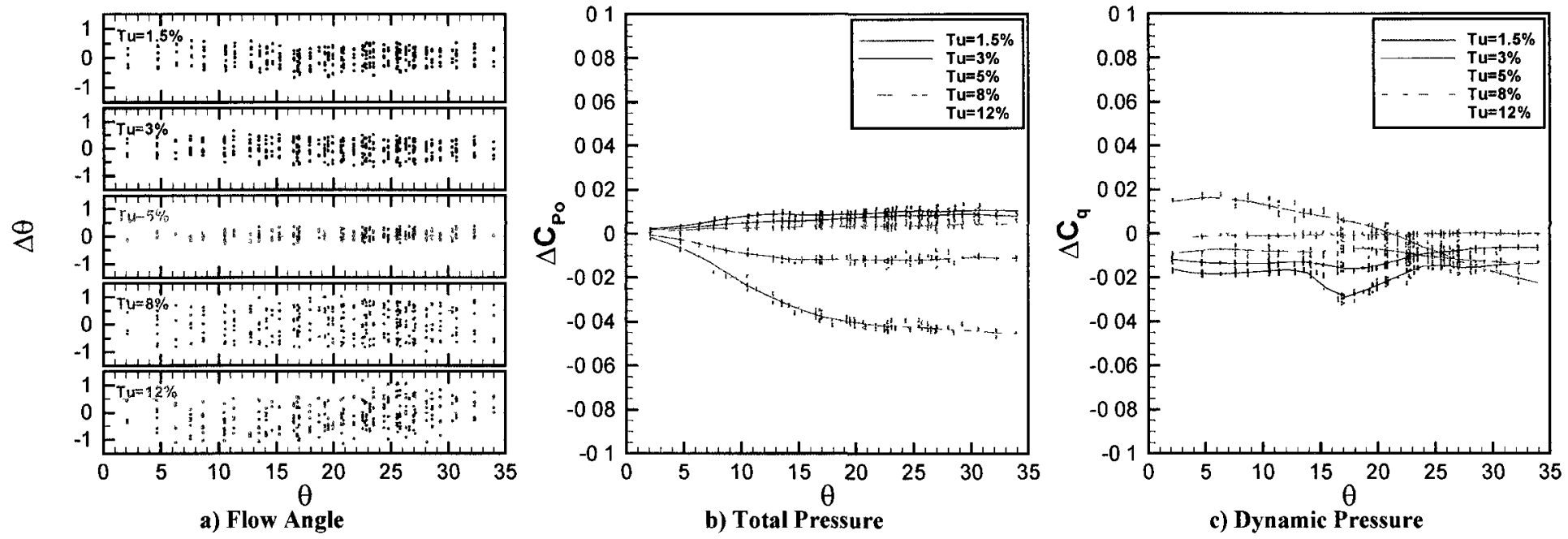

Figure D.8: Seven-Hole Pressure Probe (7HP1) Results: Calibration at $\operatorname{Re}_{\mathrm{D}}=3900, \mathrm{Tu}=5 \%$; Data at $\mathrm{Re}_{\mathrm{D}}=3900$, $\mathrm{Tu}$ as shown 

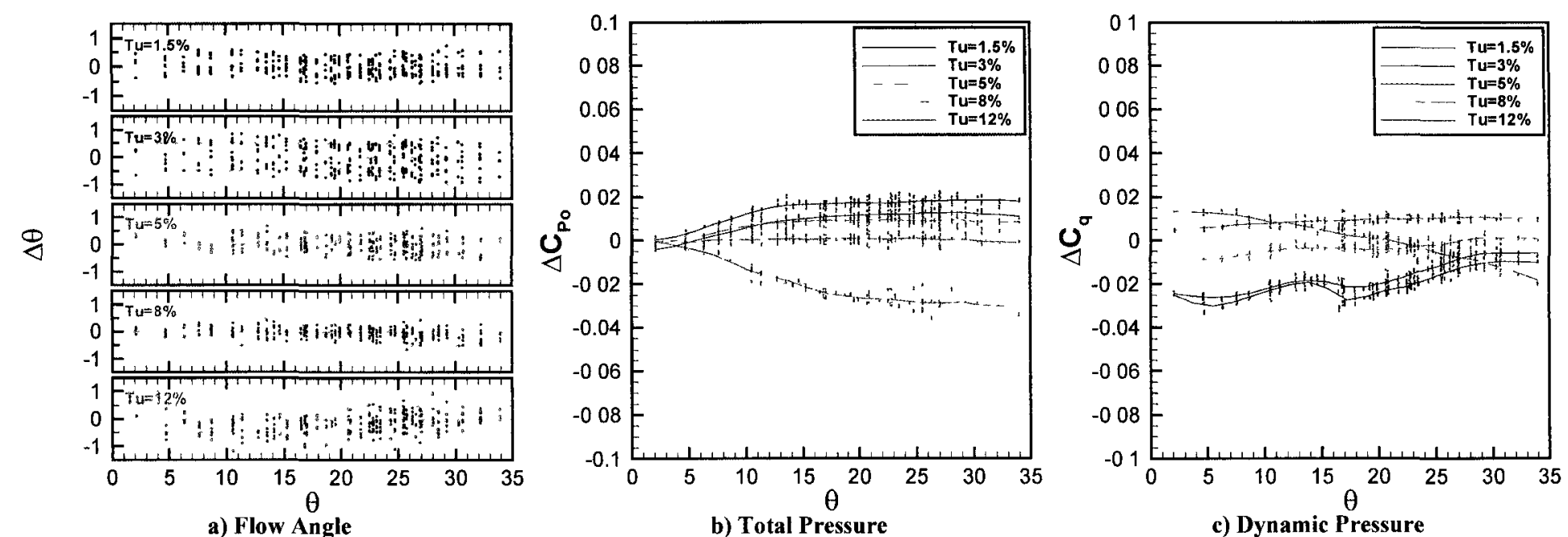

Figure D.9: Seven-Hole Pressure Probe (7HP1) Results: Calibration at $\operatorname{Re}_{\mathrm{D}}=3900, \mathrm{Tu}=8 \%$; Data at $\mathrm{Re}_{\mathrm{D}}=3900$, $\mathrm{Tu}$ as shown
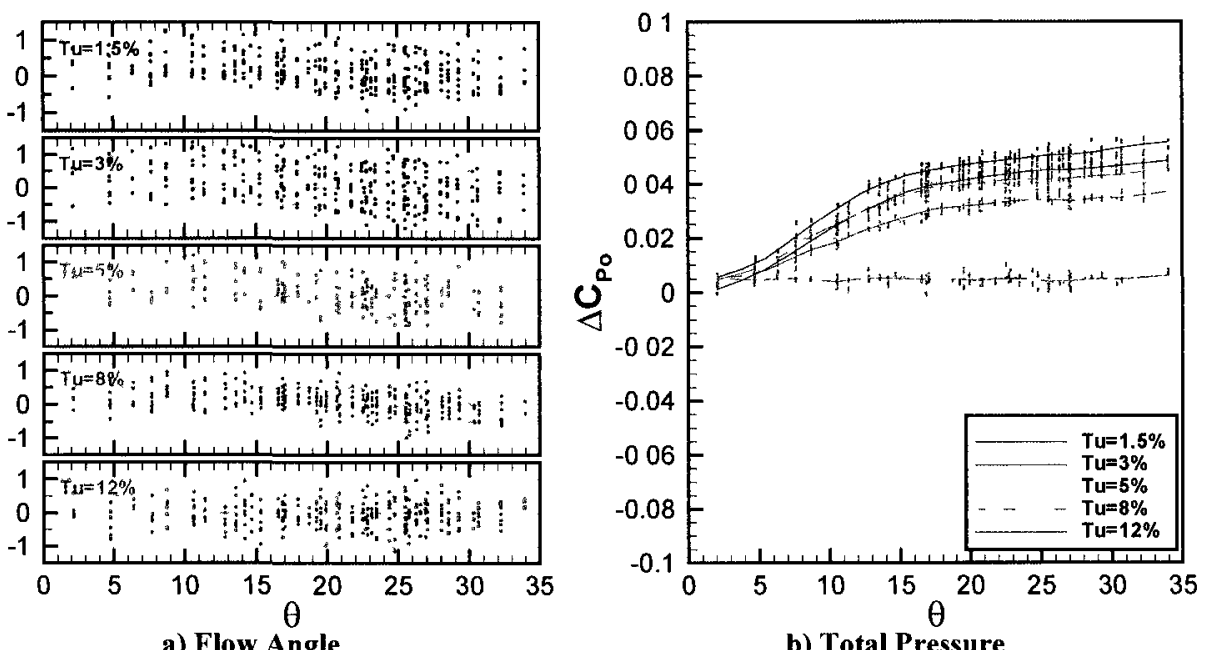

b) Total Pressure

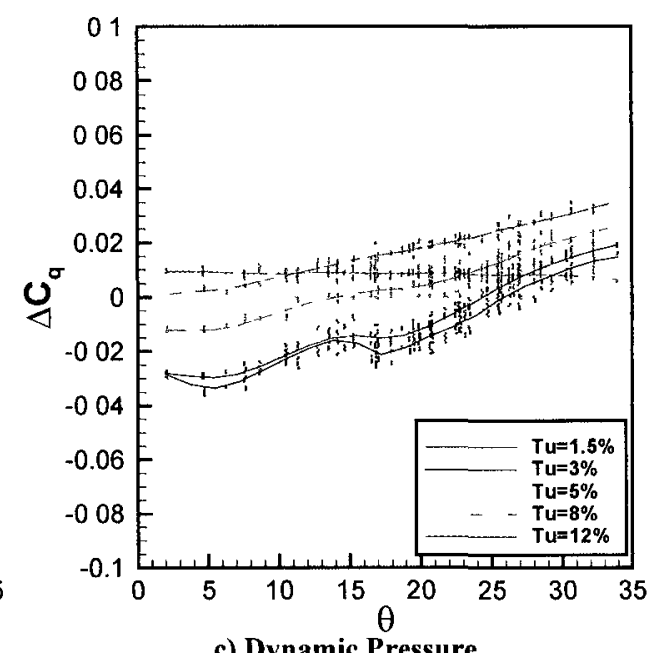

c) Dynamic Pressure

Figure D.10: Seven-Hole Pressure Probe (7HP1) Results: Calibration at $\operatorname{Re}_{\mathrm{D}}=3900, \mathrm{Tu}=12 \%$; Data at $\mathrm{Re}_{\mathrm{D}}=3900$, $\mathrm{Tu}$ as shown 


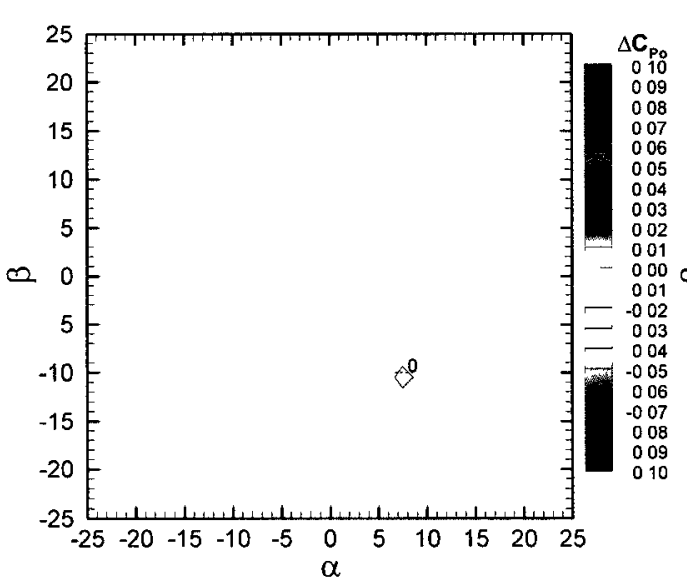

a) $\mathrm{Tu}=1.5 \%$

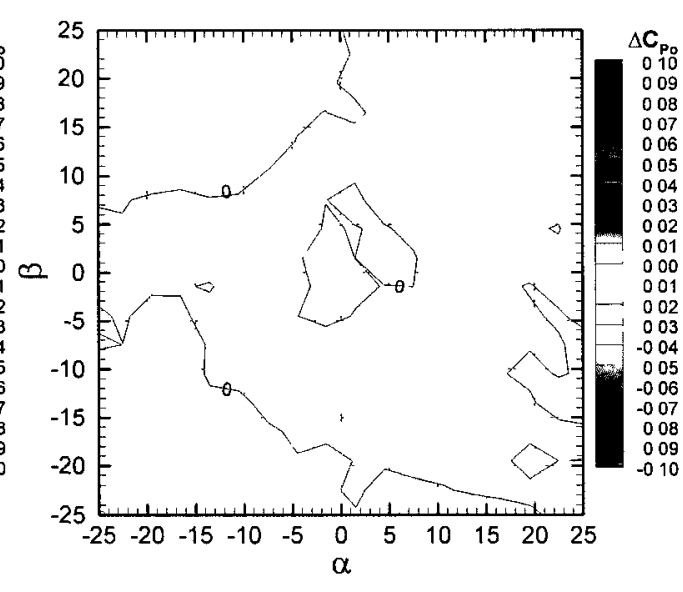

b) $\mathbf{T u}=3 \%$

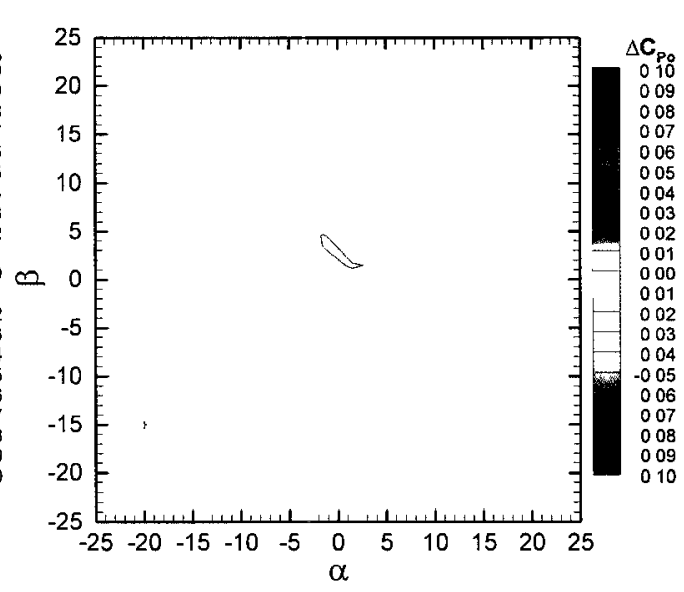

c) $\mathrm{Tu}=5 \%$

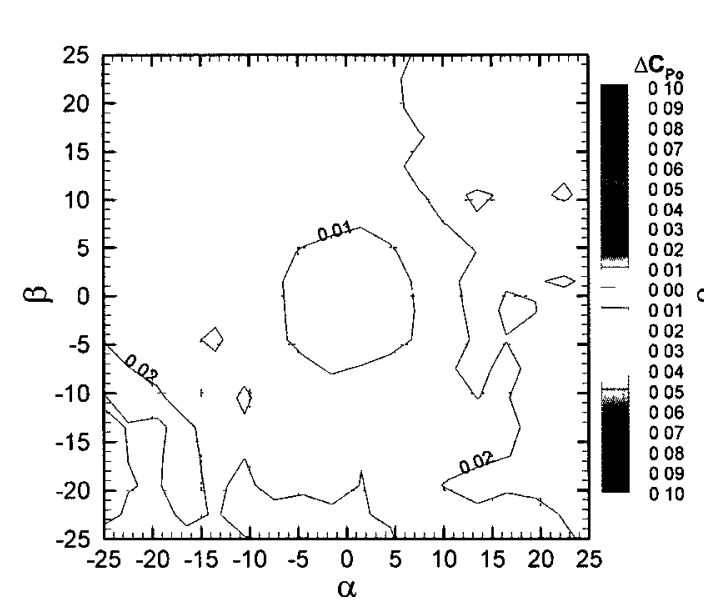

d) $\mathrm{Tu}=8 \%$

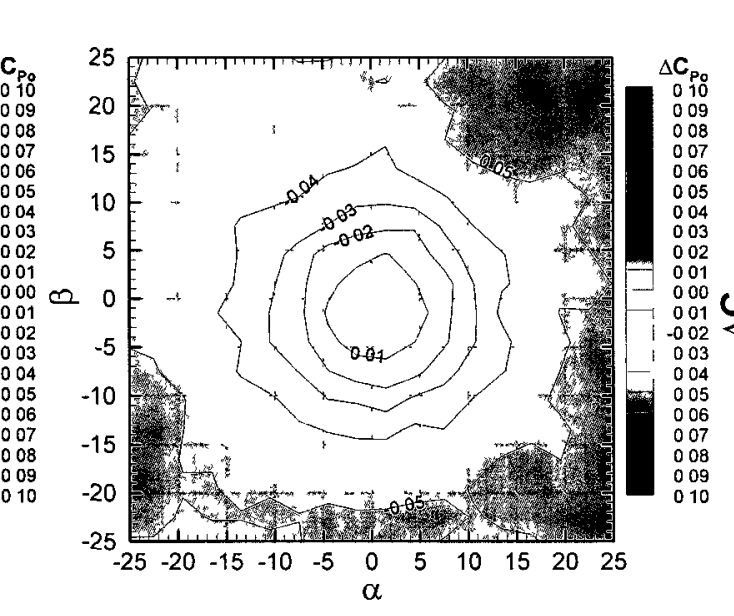

e) $\mathrm{Tu}=12 \%$

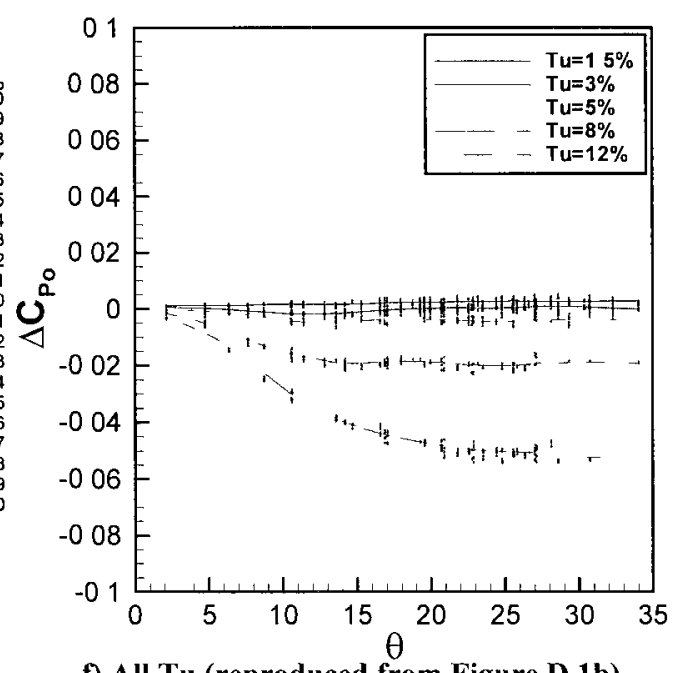

f) All Tu (reproduced from Figure D.1b)

Figure D.11: Seven-Hole Pressure Probe (7HP1) Results for Total Pressure: Calibration at $\operatorname{Re}_{\mathrm{D}}=3900, \mathrm{Tu}=1.5 \%$; $\mathrm{Data}_{\mathrm{at}} \mathrm{Re}_{\mathrm{D}}=3900$, $\mathrm{Tu}$ as shown 


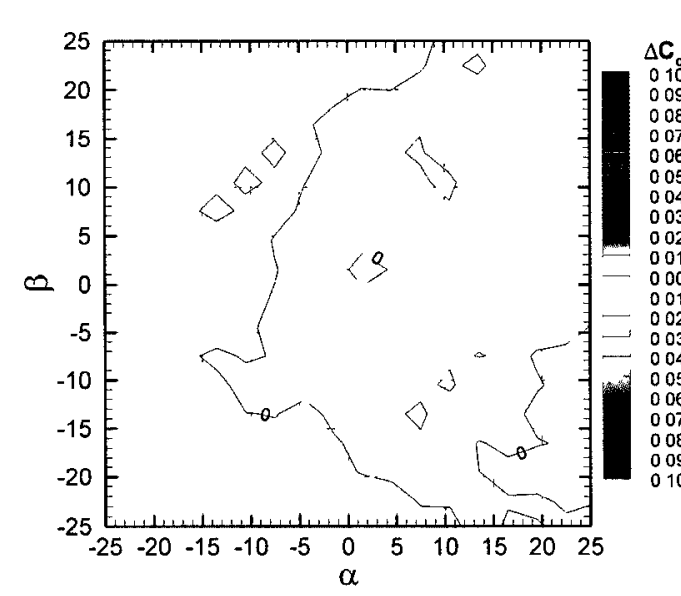

a) $\mathrm{Tu}=1.5 \%$

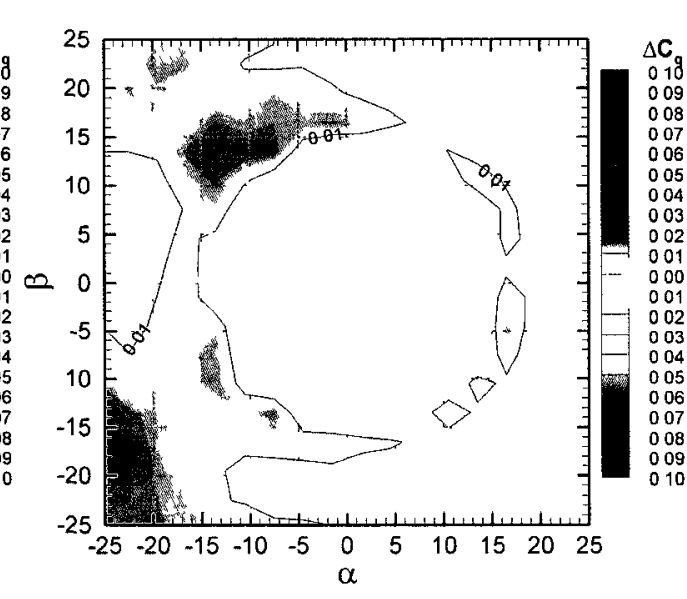

b) $\mathrm{Tu}=3 \%$

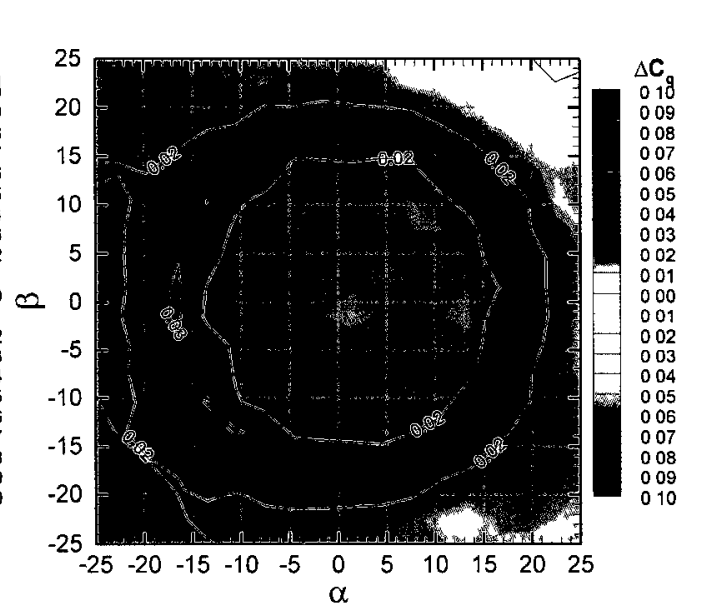

c) $\mathbf{T u}=5 \%$

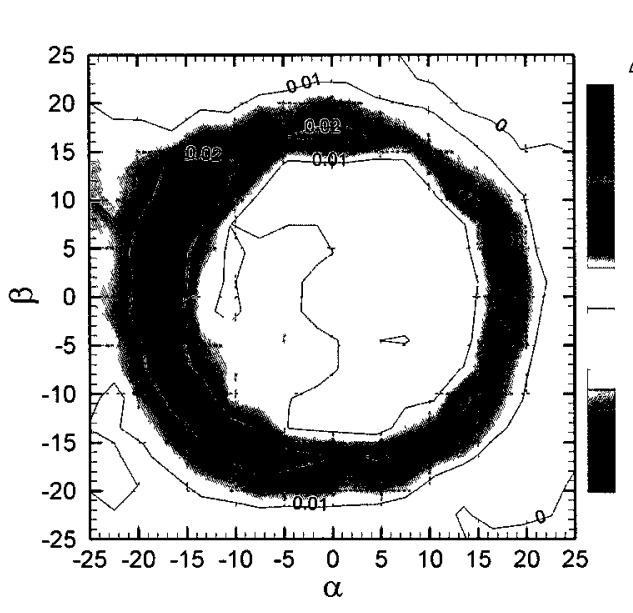

d) $\mathrm{Tu}=8 \%$

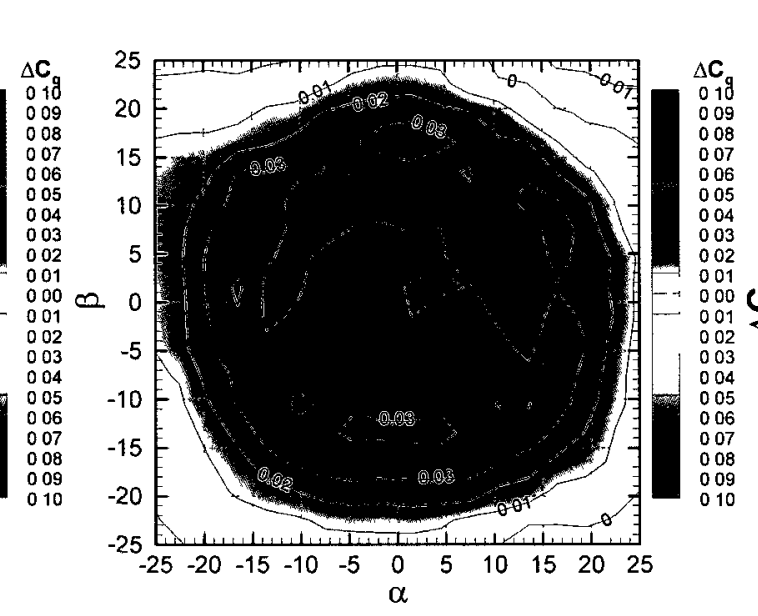

e) $\mathrm{Tu}=12 \%$

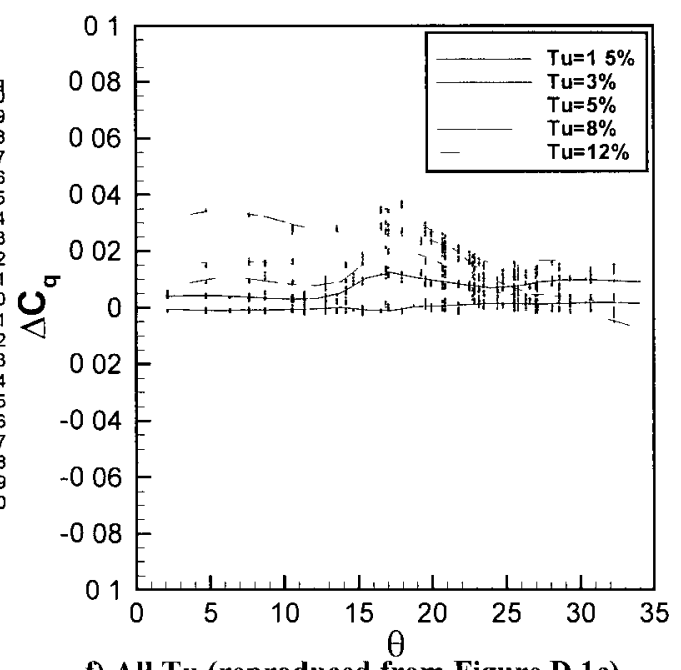

f) All Tu (reproduced from Figure D.1c)

Figure D.12: Seven-Hole Pressure Probe (7HP1) Results for Dynamic Pressure: Calibration at $\operatorname{Re}_{\mathrm{D}}=3900, \mathrm{Tu}=1.5 \%$; Data at $\mathrm{Re}_{\mathrm{D}}=3900$, Tu as shown 

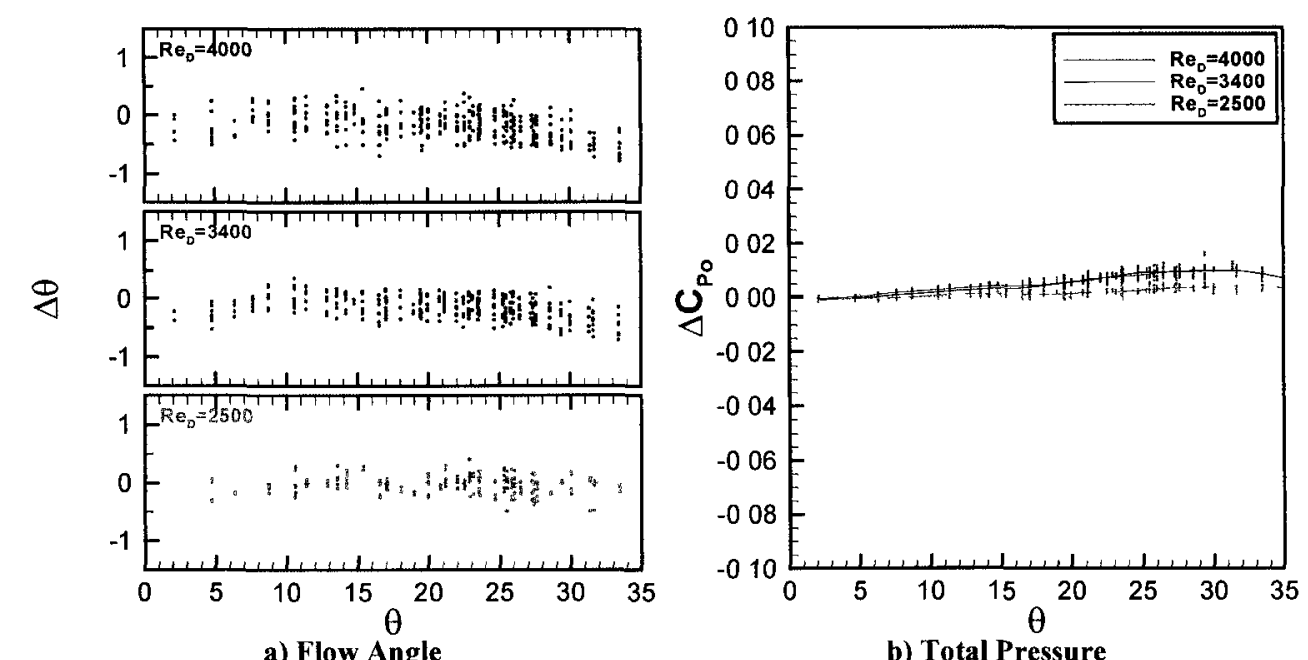

a) Flow Angle

b) Total Pressure

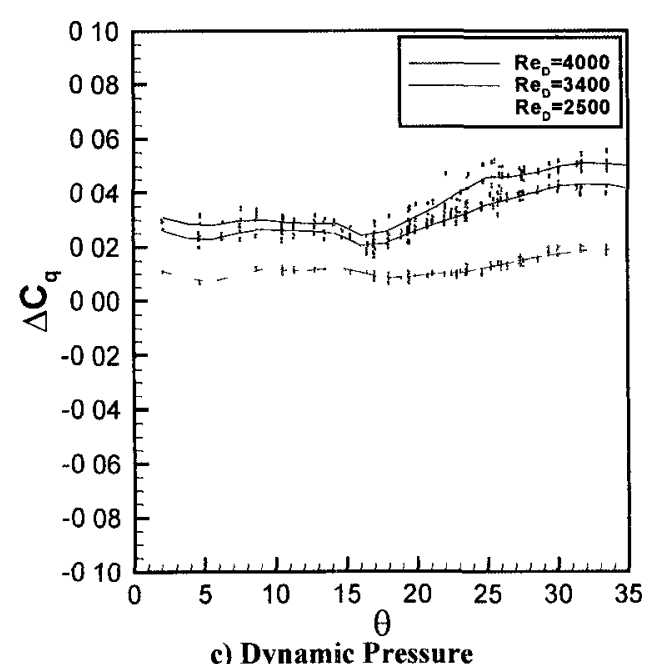

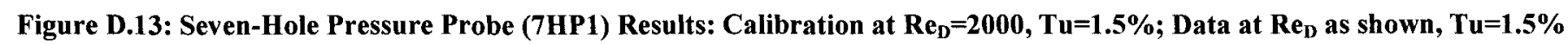
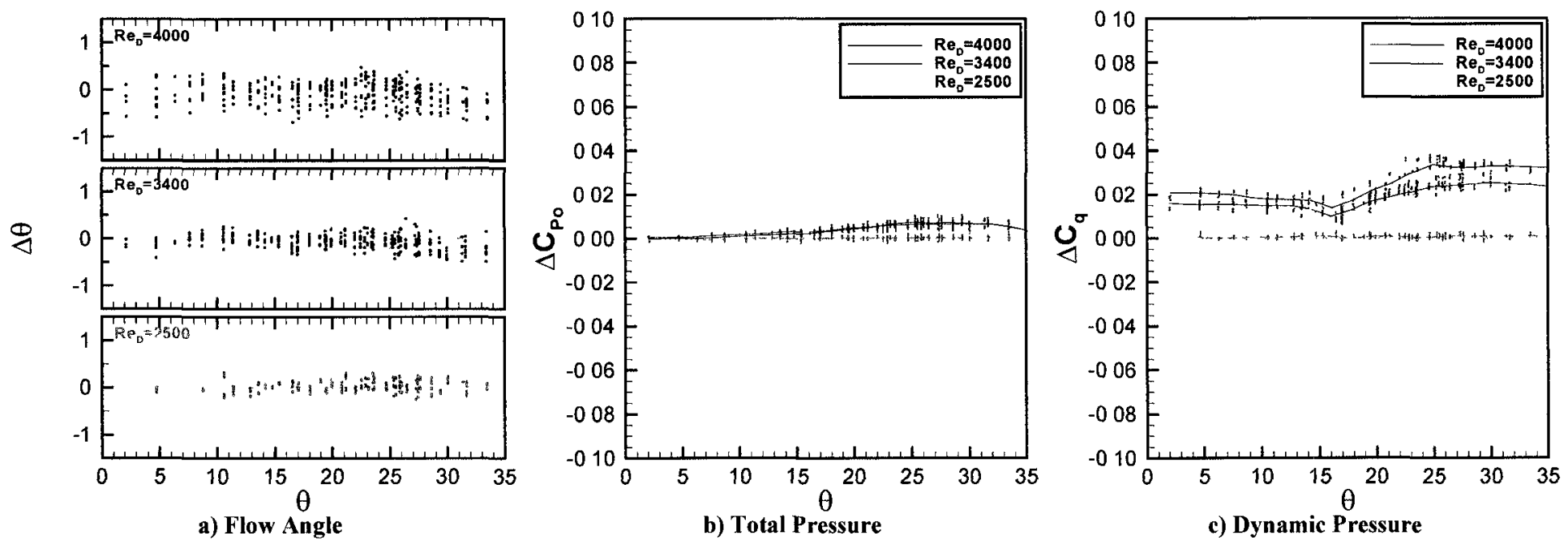

Figure D.14: Seven-Hole Pressure Probe (7HP1) Results: Calibration at $R_{D}=2500, T u=1.5 \%$; Data at $\operatorname{Re}_{\mathrm{D}}$ as shown, $\mathrm{Tu}=1.5 \%$ 

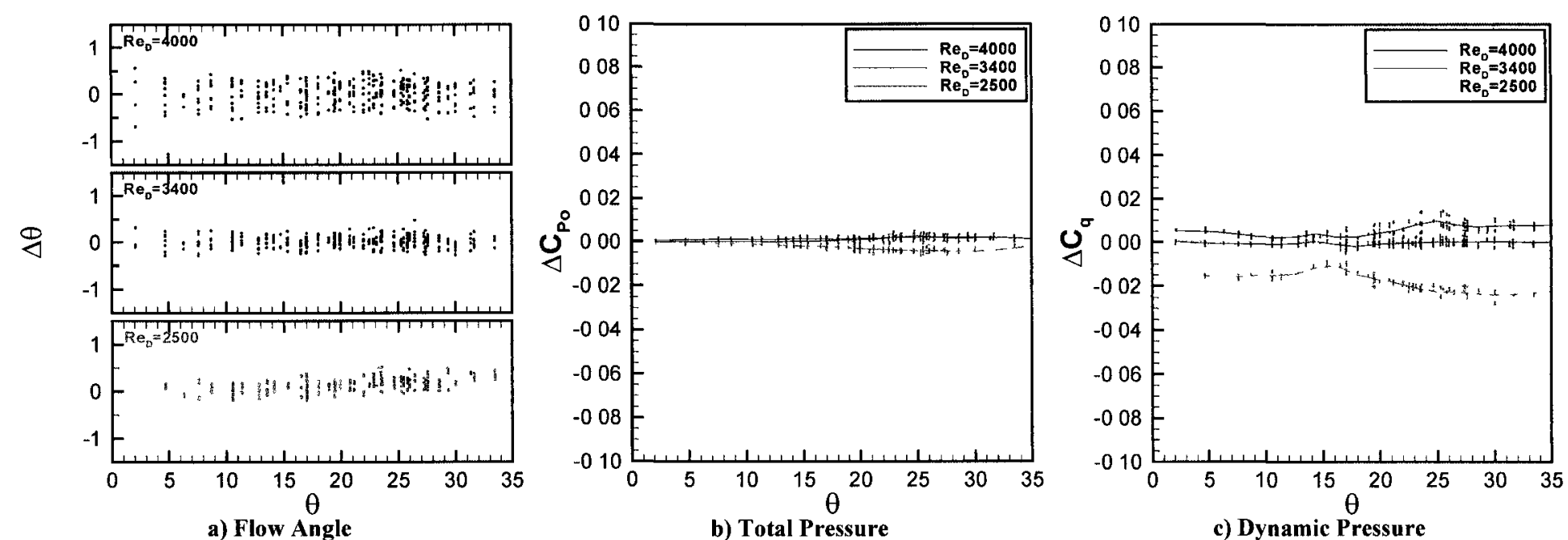

Figure D.15: Seven-Hole Pressure Probe (7HP1) Results: Calibration at $R_{\mathrm{D}}=3400, \mathrm{Tu}=1.5 \%$; Data at $\mathrm{Re}_{\mathrm{D}}$ as shown, $\mathrm{Tu}=1.5 \%$
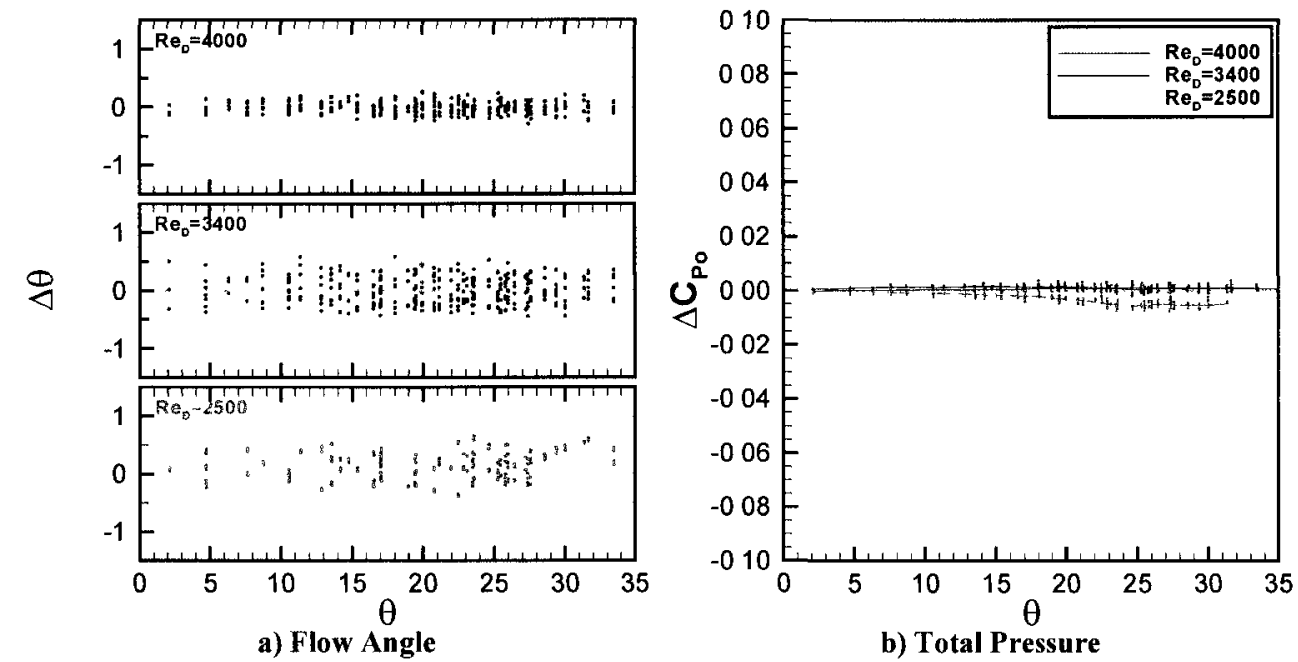

b) Total Pressure

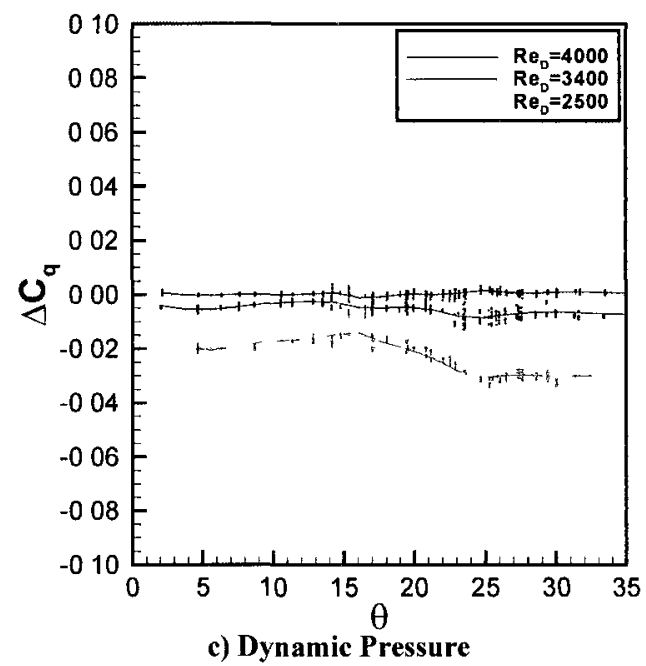

Figure D.16: Seven-Hole Pressure Probe (7HP1) Results: Calibration at $\operatorname{Re}_{\mathrm{D}}=4000, \mathrm{Tu}=1.5 \%$; Data at $\operatorname{Re}_{\mathrm{D}}$ as shown, $\mathrm{Tu}=1.5 \%$ 

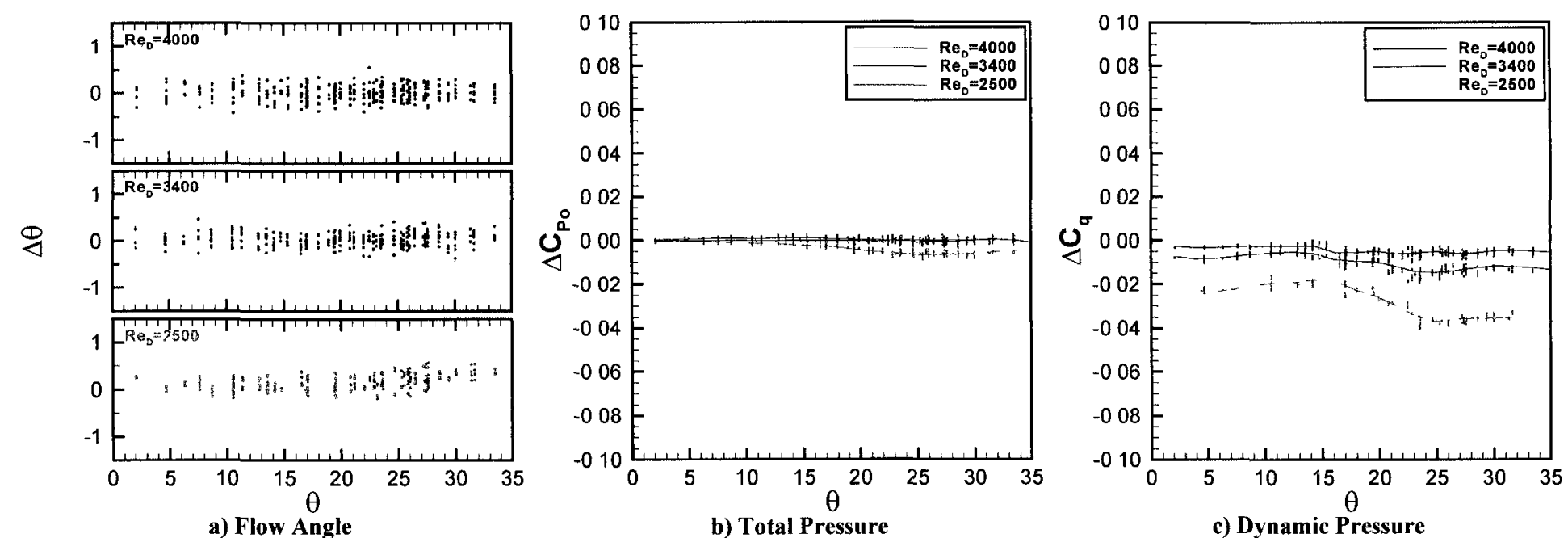

Figure D.17: Seven-Hole Pressure Probe (7HP1) Results: Calibration at $\operatorname{Re}_{D}=4400, T u=1.5 \%$; Data at $\operatorname{Re}_{D}$ as shown, $T u=1.5 \%$
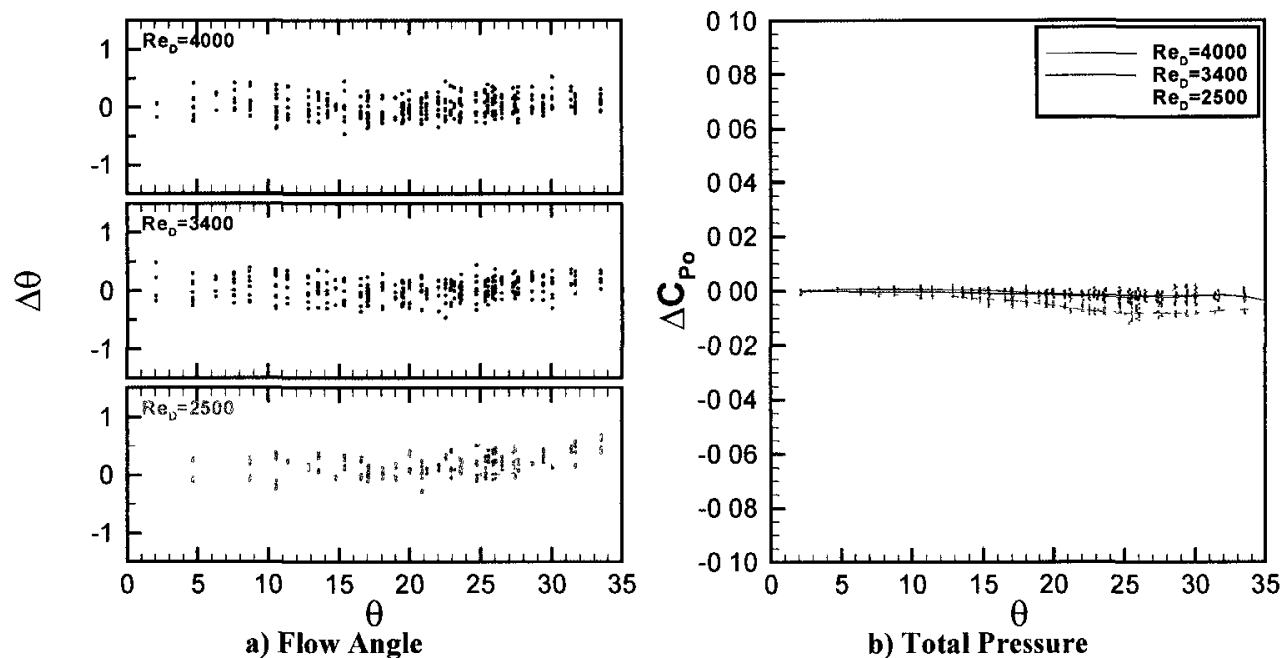

b) Total Pressure

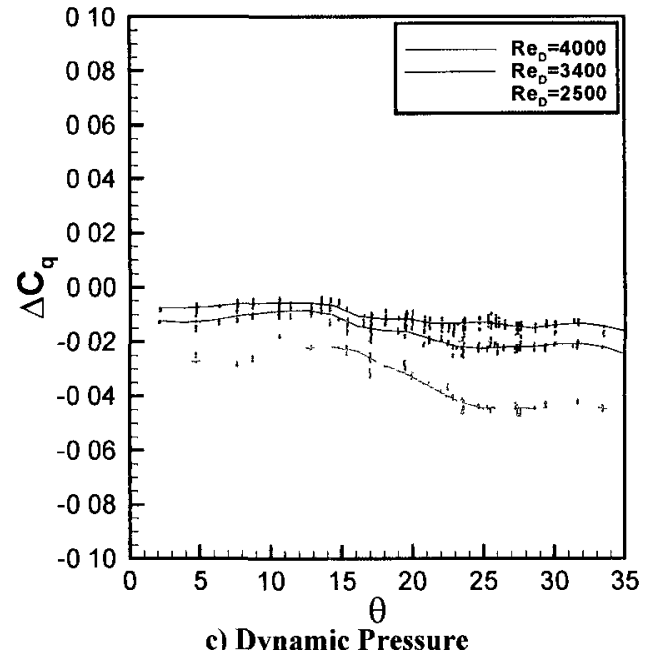

Figure D.18: Seven-Hole Pressure Probe (7HP1) Results: Calibration at $\operatorname{Re}_{\mathrm{D}}=5150, \mathrm{Tu}=1.5 \%$; Data at $\mathrm{Re}_{\mathrm{D}}$ as shown, $\mathrm{Tu}=1.5 \%$ 


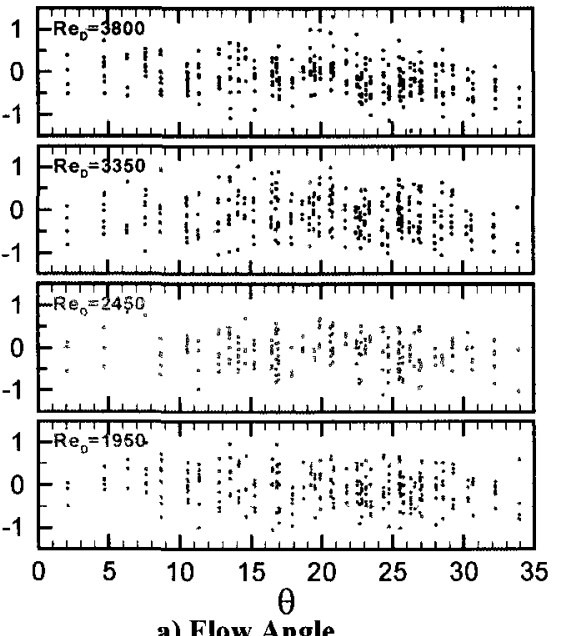

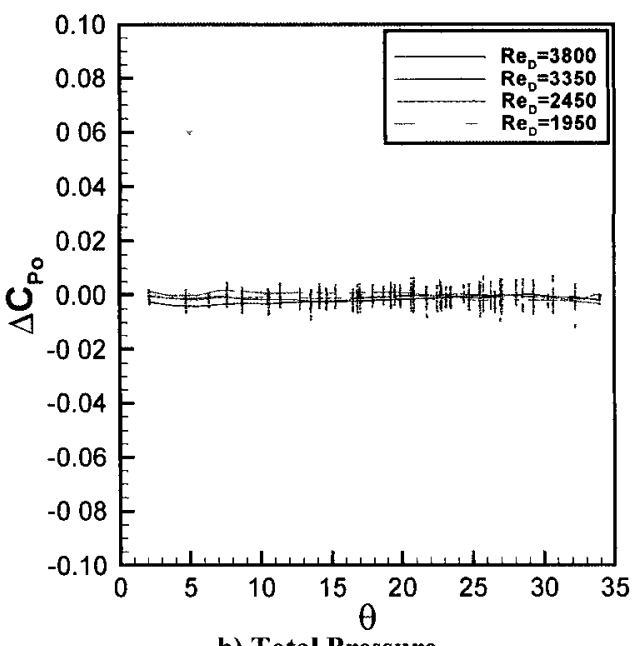

b) Total Pressure

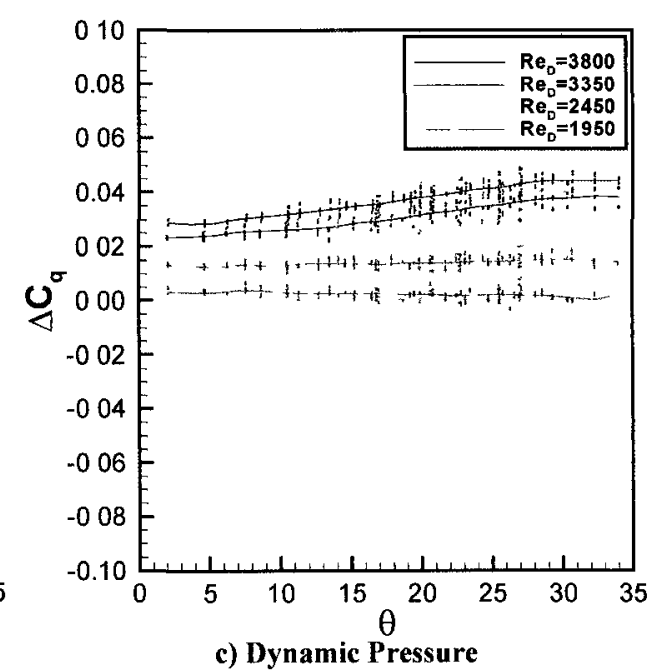

c) Dynamic Pressure

Figure D.19: Seven-Hole Pressure Probe (7HP1) Results: Calibration at $\operatorname{Re}_{\mathrm{D}}=1950, \mathrm{Tu}=12 \%$; Data at $\mathrm{Re}_{\mathrm{D}}$ as shown, $\mathrm{Tu}=12 \%$

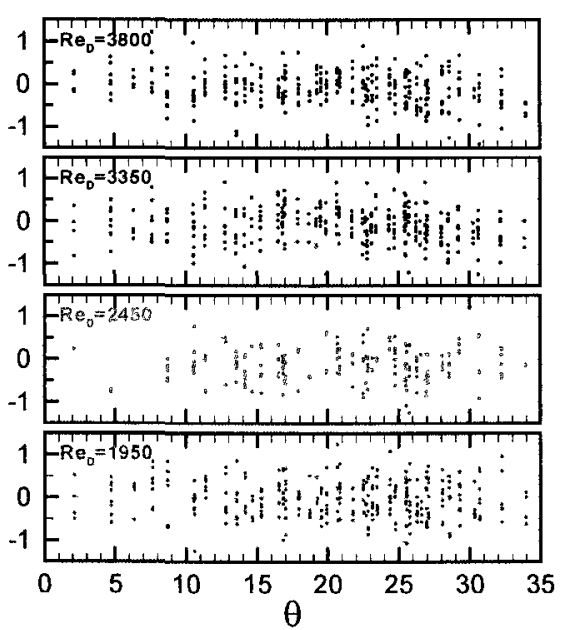

a) Flow Angle

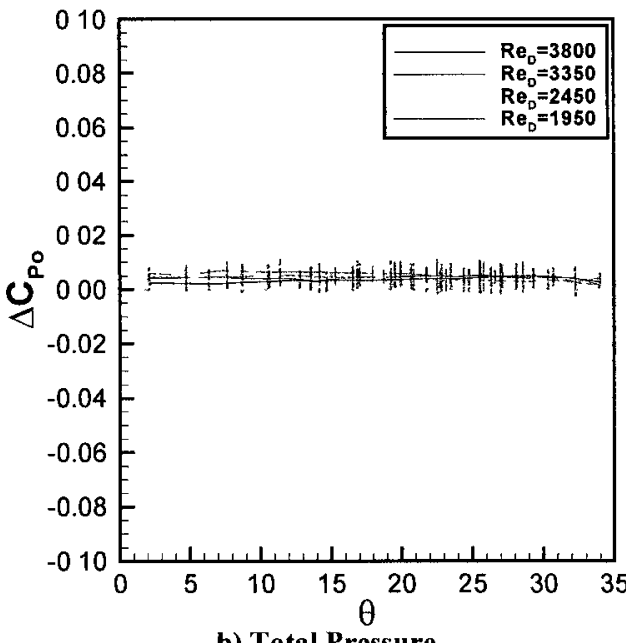

b) Total Pressure

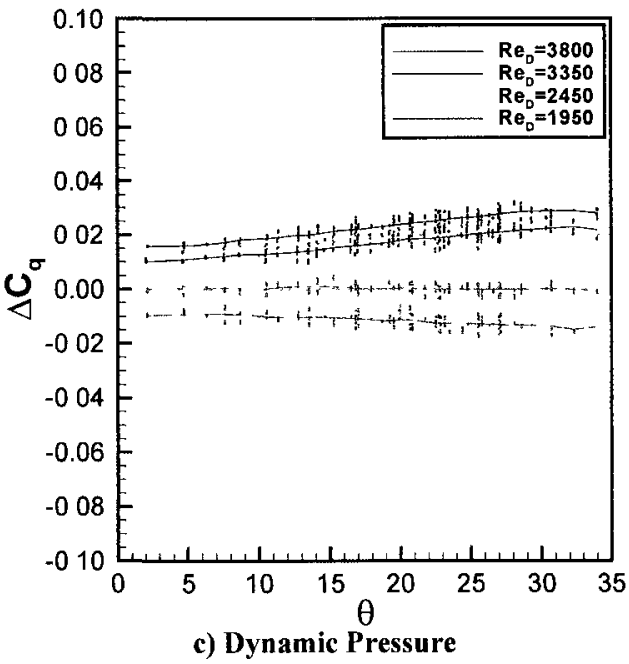

c) Dynamic Pressure

Figure D.20: Seven-Hole Pressure Probe (7HP1) Results: Calibration at $\operatorname{Re}_{\mathrm{D}}=\mathbf{2 4 5 0}, \mathrm{Tu}=12 \%$; Data at $\mathrm{Re}_{\mathrm{D}}$ as shown, $\mathrm{Tu}=12 \%$ 
g

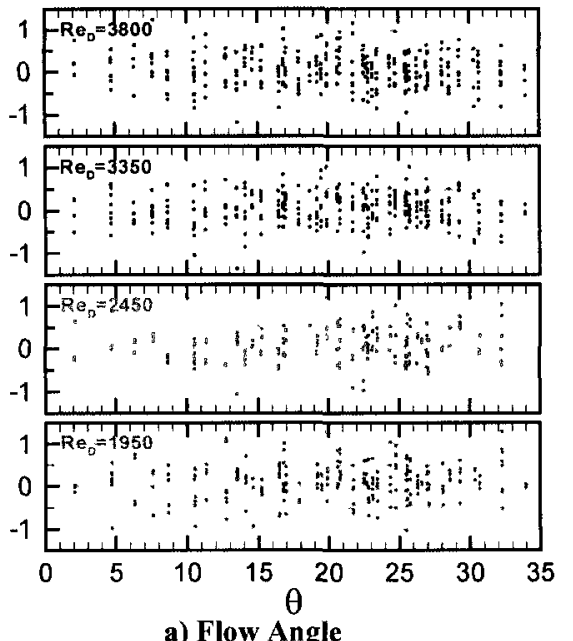

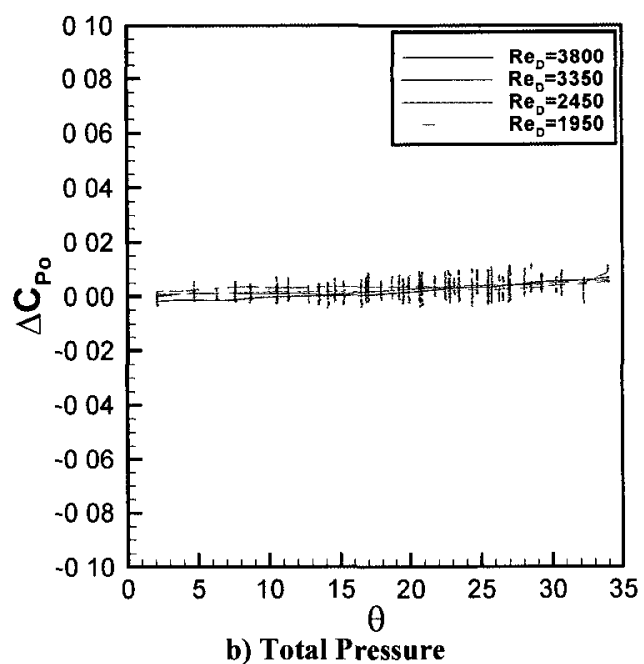

b) Total Pressure

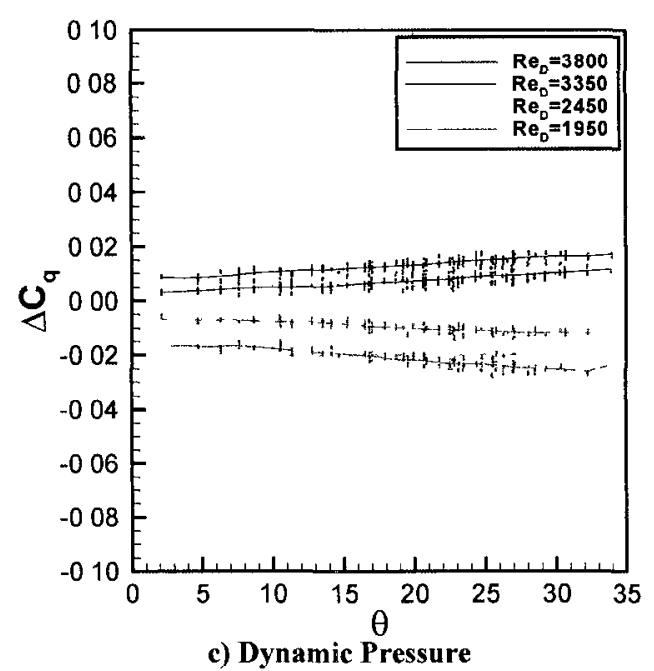

c) Dynamic Pressure

Figure D.21: Seven-Hole Pressure Probe (7HP1) Results: Calibration at $R_{D}=2850, T u=12 \%$; Data at $R_{D}$ as shown, $T u=12 \%$

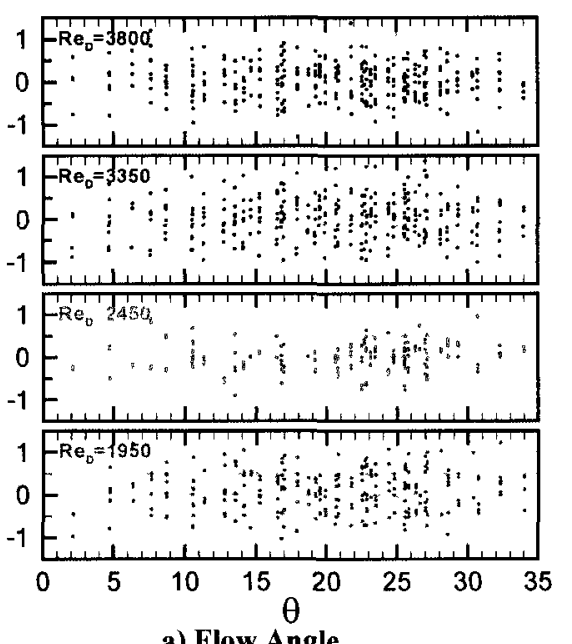

a) Flow Angle

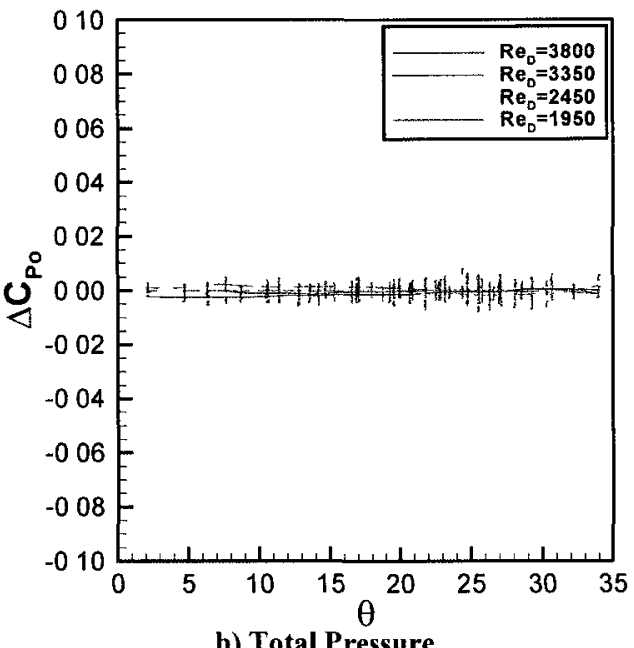

b) Total Pressure

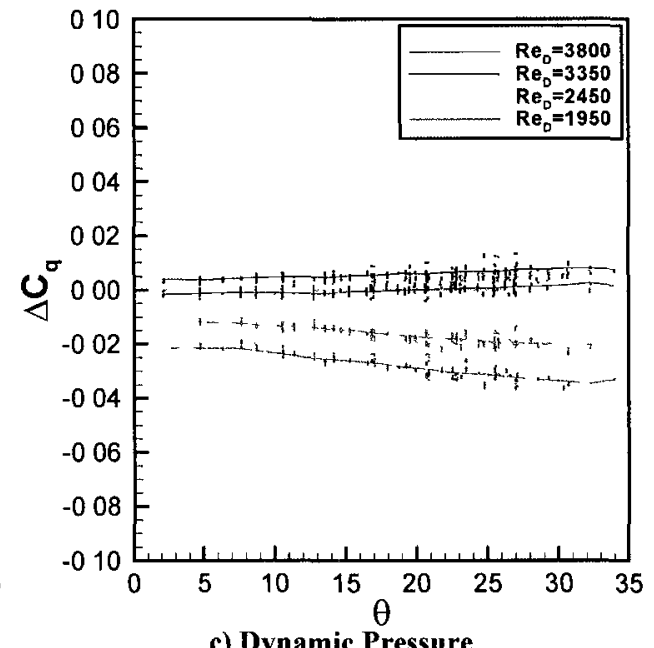

c) Dynamic Pressure

Figure D.22: Seven-Hole Pressure Probe (7HP1) Results: Calibration at $\operatorname{Re}_{\mathrm{D}}=3400, \mathrm{Tu}=12 \%$; Data at $\operatorname{Re}_{\mathrm{D}}$ as shown, $\mathrm{Tu}=12 \%$ 

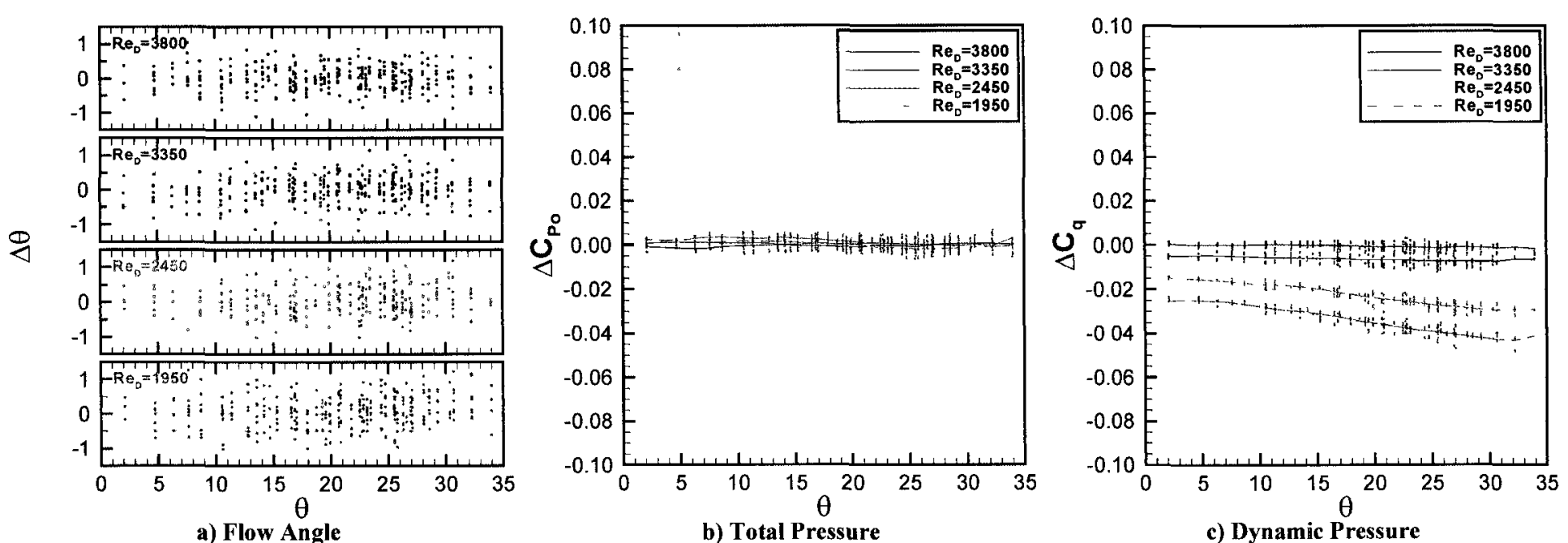

Figure D.23: Seven-Hole Pressure Probe (7HP1) Results: Calibration at $\operatorname{Re}_{\mathrm{D}}=3800, \mathrm{Tu}=12 \%$; Data at $\mathrm{Re}_{\mathrm{D}}$ as shown, $\mathrm{Tu}=12 \%$ 


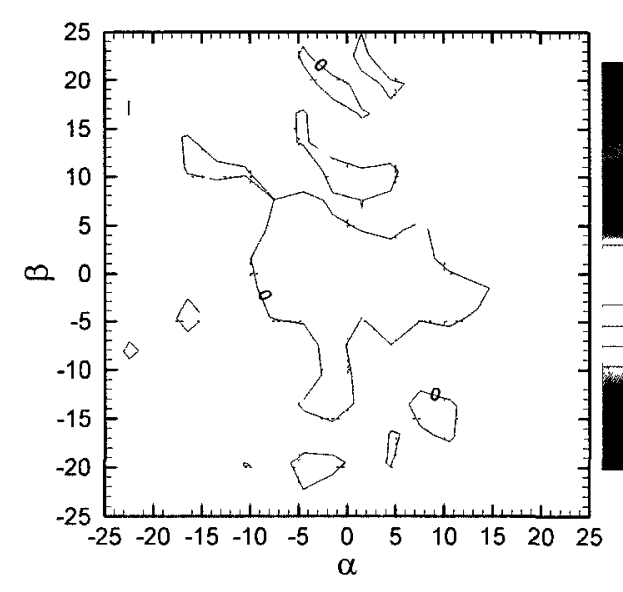

a) $\operatorname{Re}_{\mathrm{D}}=\mathbf{2 5 0 0}$

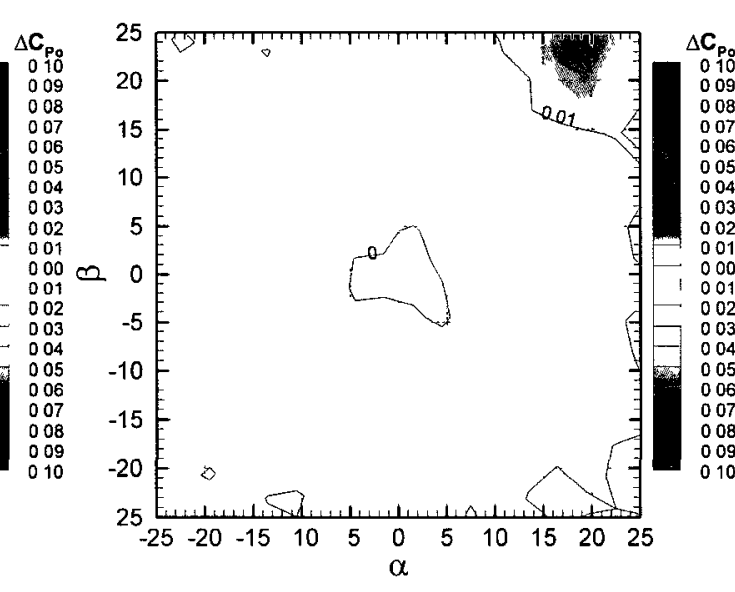

b) $\operatorname{Re}_{\mathrm{D}}=3400$

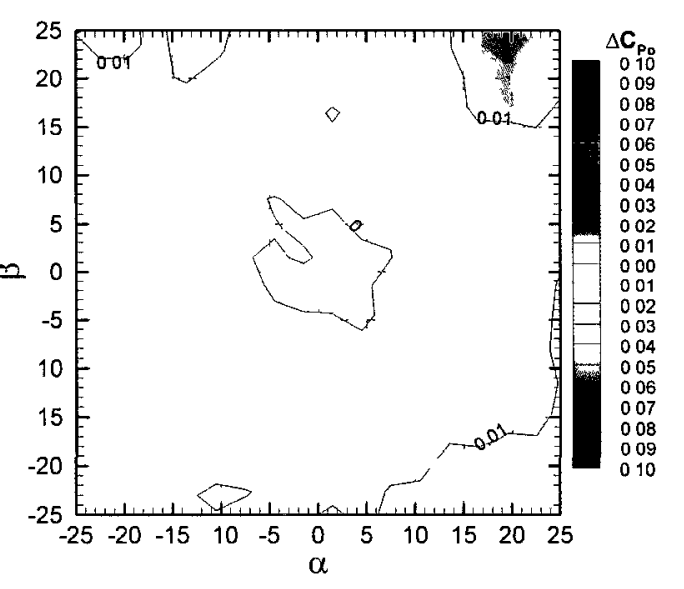

c) $\operatorname{Re}_{\mathrm{D}}=\mathbf{4 0 0 0}$

Figure D.24: Seven-Hole Pressure Probe (7HP1) Results for Total Pressure: Calibration at $\operatorname{Re}_{\mathrm{D}}=\mathbf{2 0 0 0 ,} \mathbf{T} u=1.5 \%$ Data at $\operatorname{Re}_{\mathrm{D}}$ as shown, $\mathrm{Tu}=1.5 \%$

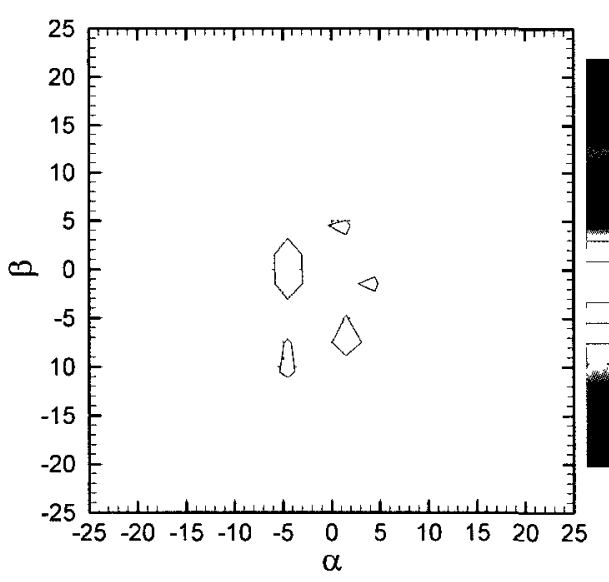

a) $\operatorname{Re}_{\mathrm{D}}=\mathbf{2 5 0 0}$

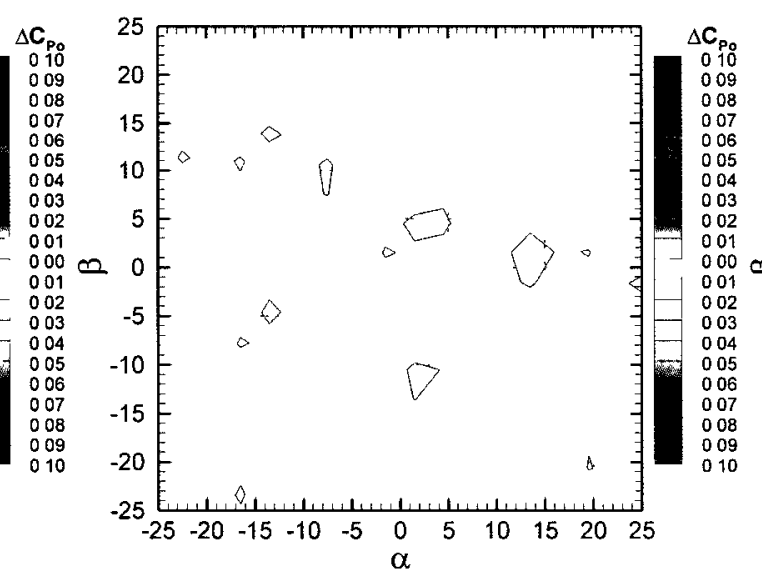

b) $\operatorname{Re}_{\mathrm{D}}=\mathbf{3 4 0 0}$

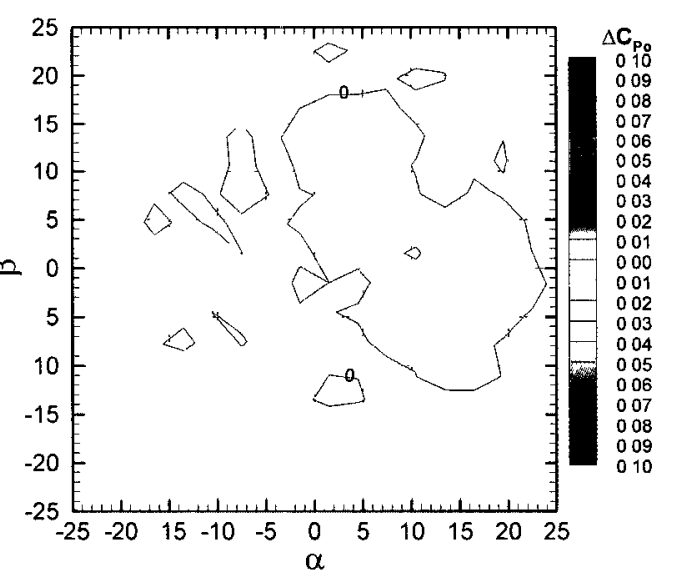

c) $\operatorname{Re}_{\mathrm{D}}=\mathbf{4 0 0 0}$

Figure D.25: Seven-Hole Pressure Probe (7HP1) Results for Total Pressure: Calibration at $R_{D}=3400, T u=1.5 \%$; Data $_{\text {at }} R_{D}$ as shown, $\mathrm{Tu}=\mathbf{1 . 5 \%}$ 


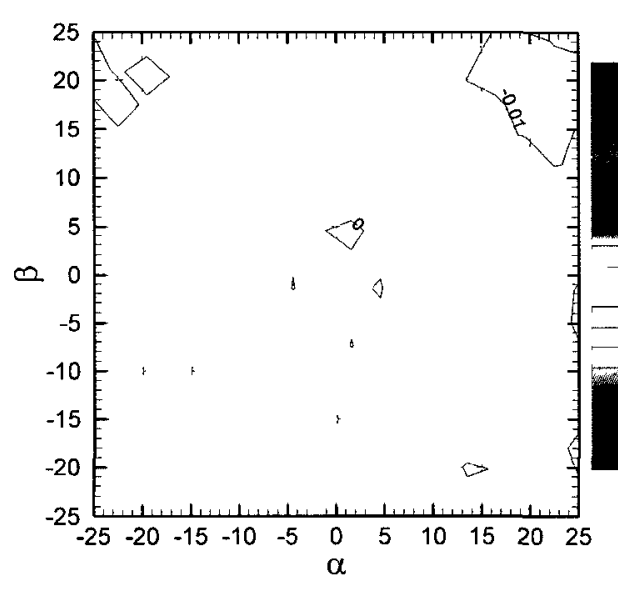

a) $\operatorname{Re}_{\mathrm{D}}=\mathbf{2 5 0 0}$
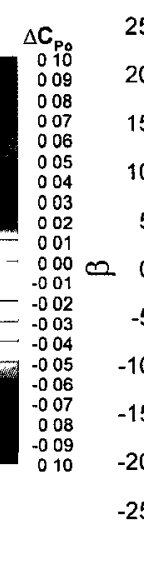

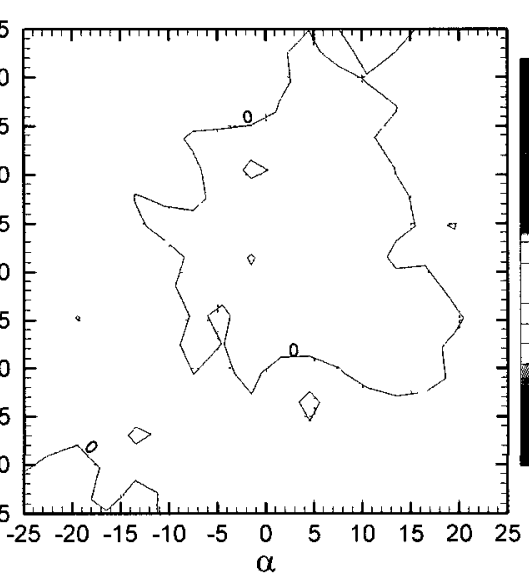

b) $\operatorname{Re}_{\mathrm{D}}=3400$

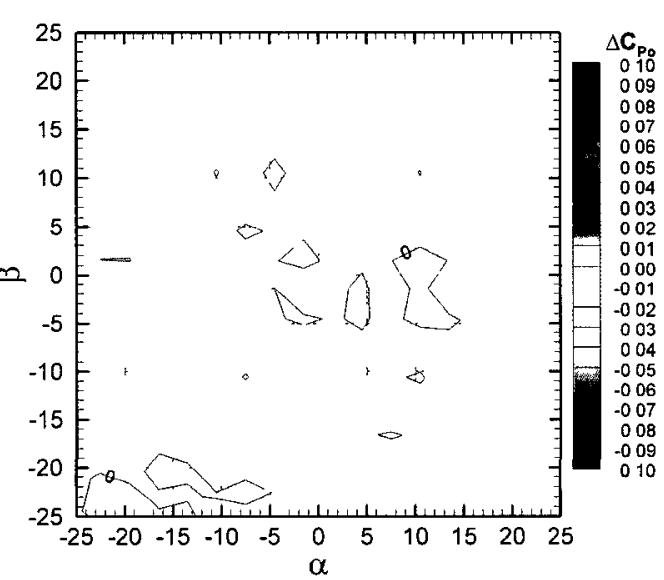

c) $\operatorname{Re}_{\mathrm{D}}=\mathbf{4 0 0 0}$

Figure D.26: Seven-Hole Pressure Probe (7HP1) Results for Total Pressure: Calibration at $\operatorname{Re}_{D}=\mathbf{5 1 5 0 ,}$ Tu=1.5\%; Data at $\mathbf{R e}_{\mathrm{D}}$ as shown, $\mathrm{Tu}=1.5 \%$

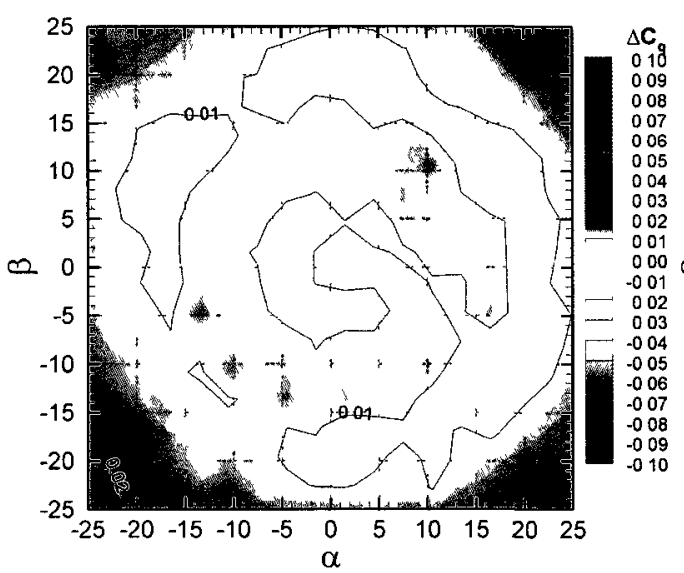

a) $\mathrm{Re}_{\mathrm{D}}=\mathbf{2 5 0 0}$

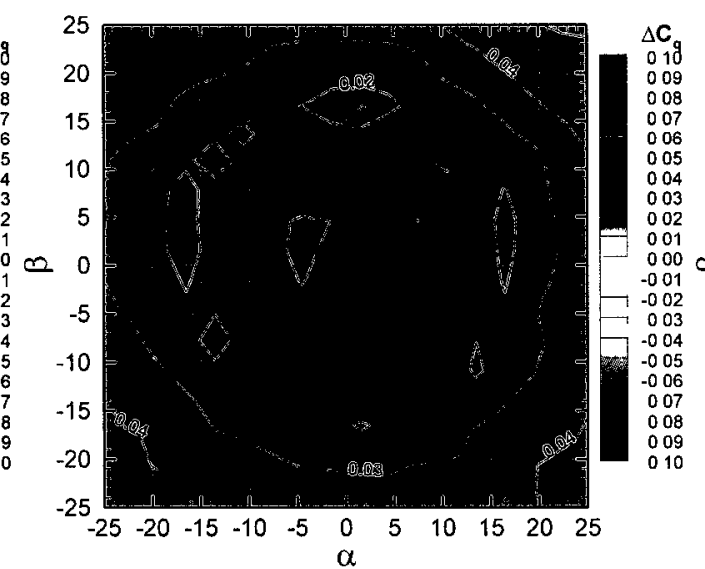

b) $\operatorname{Re}_{\mathrm{D}}=3400$

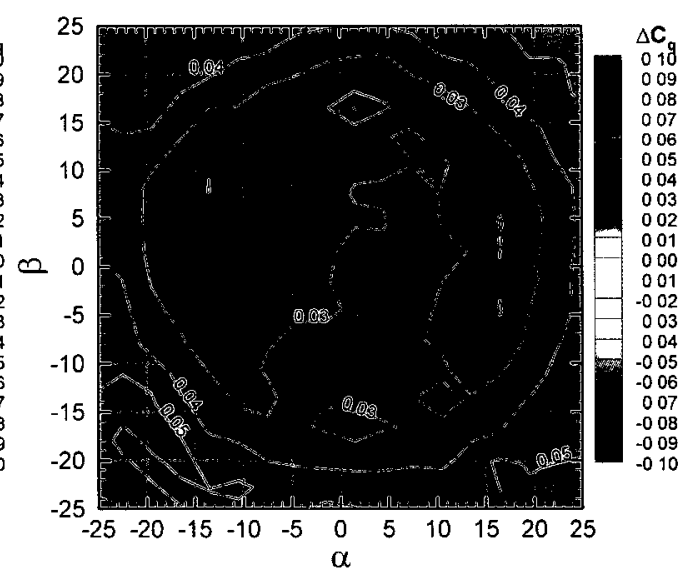

c) $\mathrm{Re}_{\mathrm{p}}=4000$

Figure D.27: Seven-Hole Pressure Probe (7HP1) Results for Dynamic Pressure: Calibration at $\operatorname{Re}_{D}=2000, T_{u}=1.5 \% ; D a t a$ at $\operatorname{Re}_{D}$ as shown, $\mathrm{Tu}=1.5 \%$ 


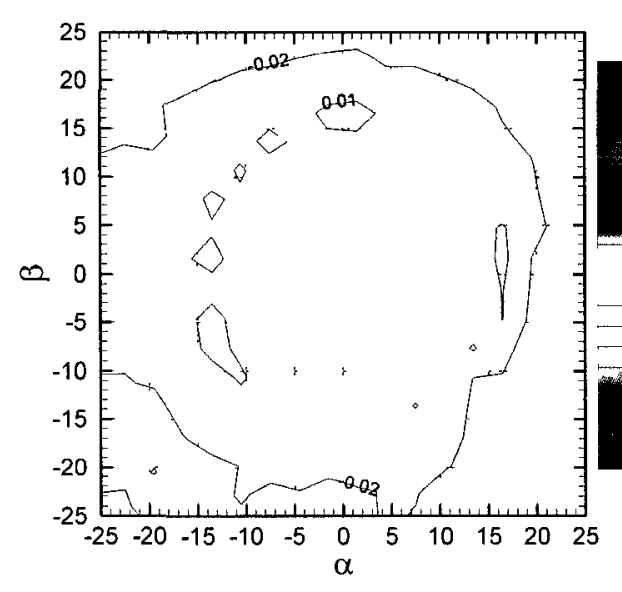

a) $\operatorname{Re}_{\mathrm{D}}=2500$

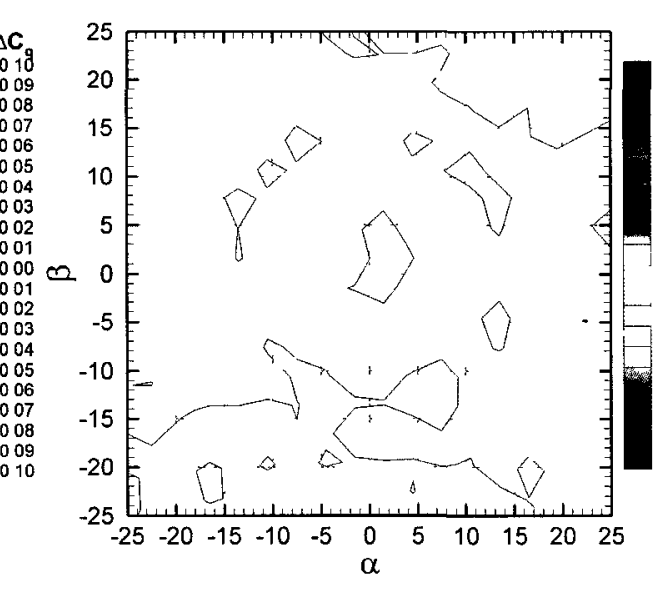

b) $\operatorname{Re}_{\mathrm{D}}=3400$

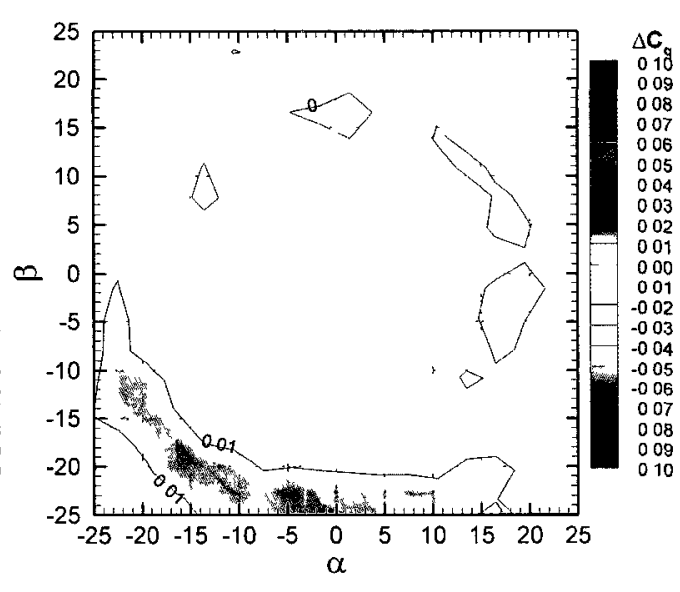

c) $\operatorname{Re}_{\mathrm{D}}=4000$

Figure D.28: Seven-Hole Pressure Probe (7HP1) Results for Dynamic Pressure: Calibration at $\operatorname{Re}_{D}=3400, T u=1.5 \%$; $D a t a$ at $R e_{D}$ as shown, $\mathrm{Tu}=1.5 \%$

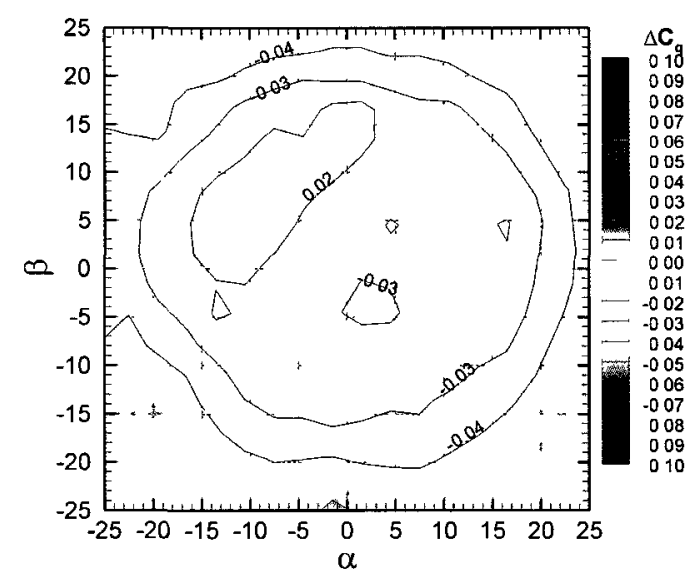

a) $\mathrm{Re}_{\mathrm{D}}=\mathbf{2 5 0 0}$

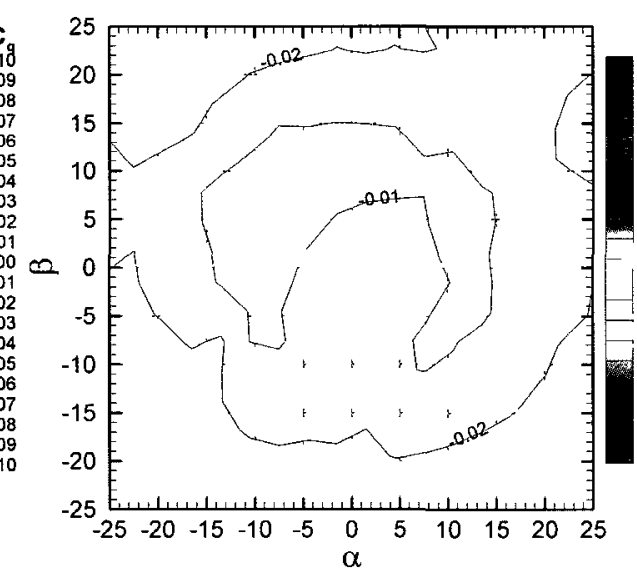

b) $\operatorname{Re}_{\mathrm{D}}=3400$

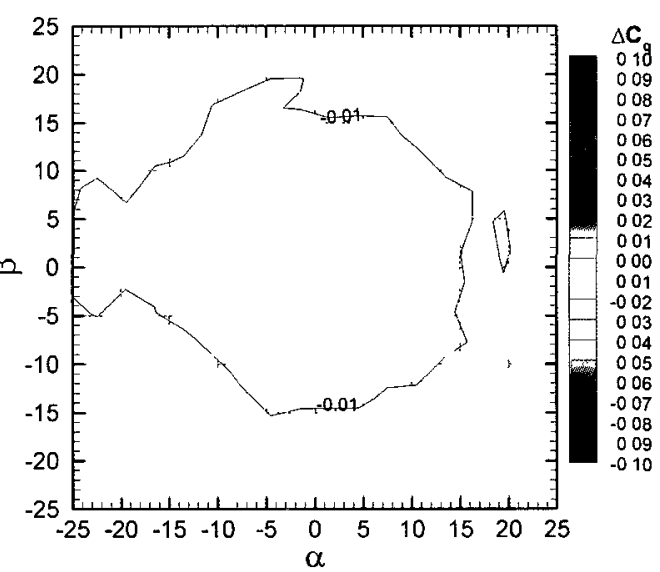

c) $\operatorname{Re}_{\mathrm{D}}=4000$

Figure D.29: Seven-Hole Pressure Probe (7HP1) Results for Dynamic Pressure: Calibration at $\operatorname{Re}_{\mathrm{D}}=\mathbf{5 1 5 0 , T u = 1 . 5 \%} \mathbf{D a t a}$ at $\mathrm{Re}_{\mathrm{D}}$ as shown, $\mathrm{Tu}=1.5 \%$ 

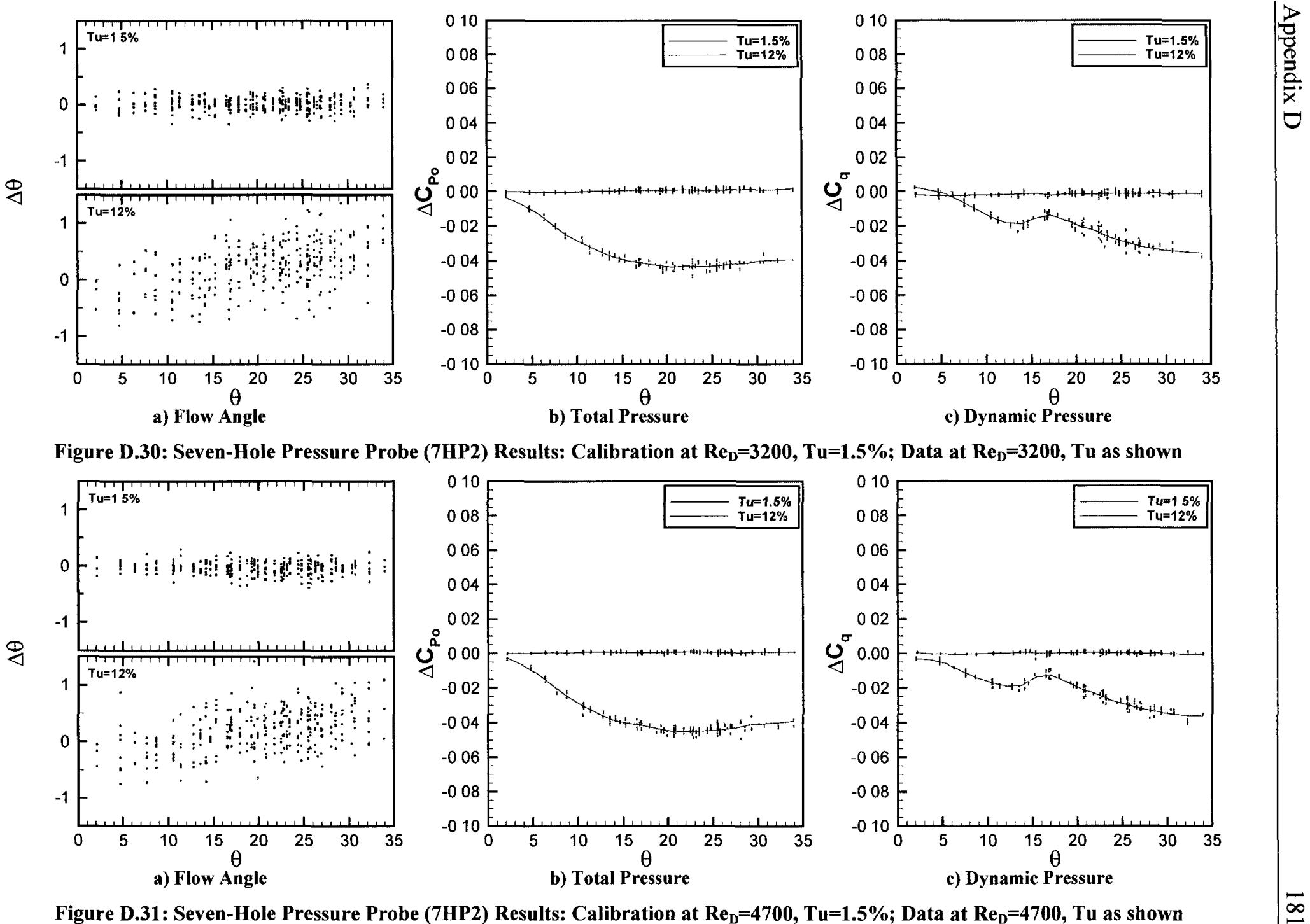

Figure D.30: Seven-Hole Pressure Probe (7HP2) Results: Calibration at $R_{D}=3200, T u=1.5 \%$; Data at $R_{D}=3200$, $T u$ as shown
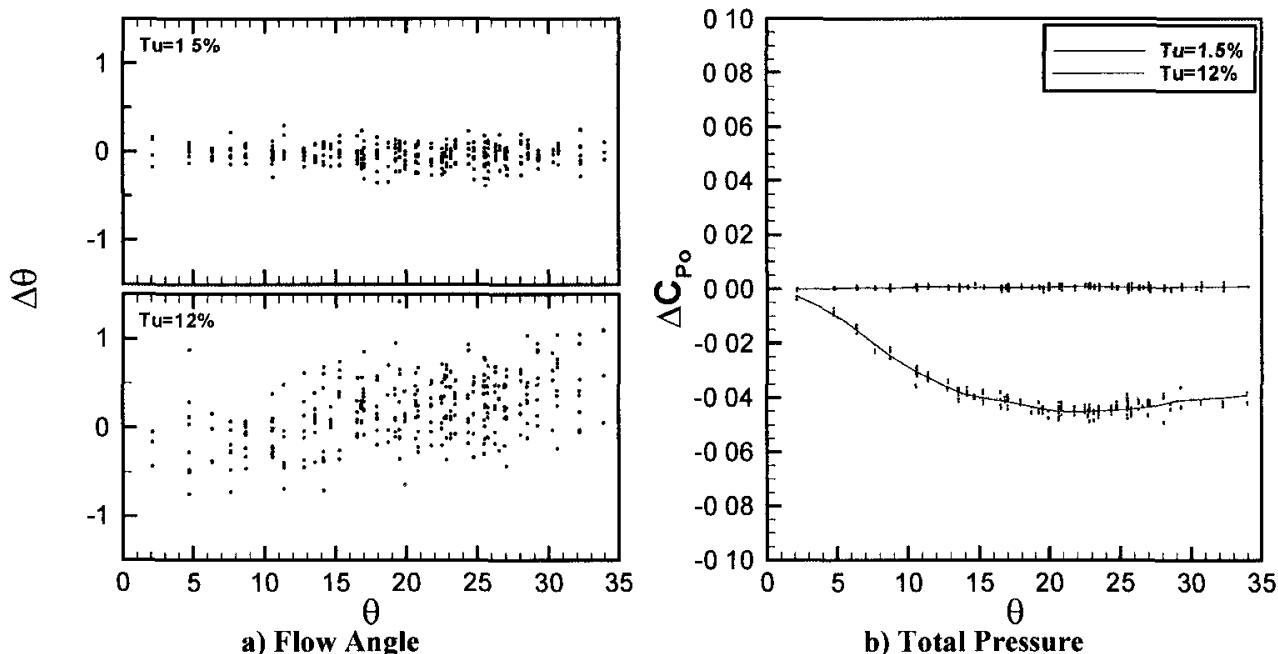

b) Total Pressure

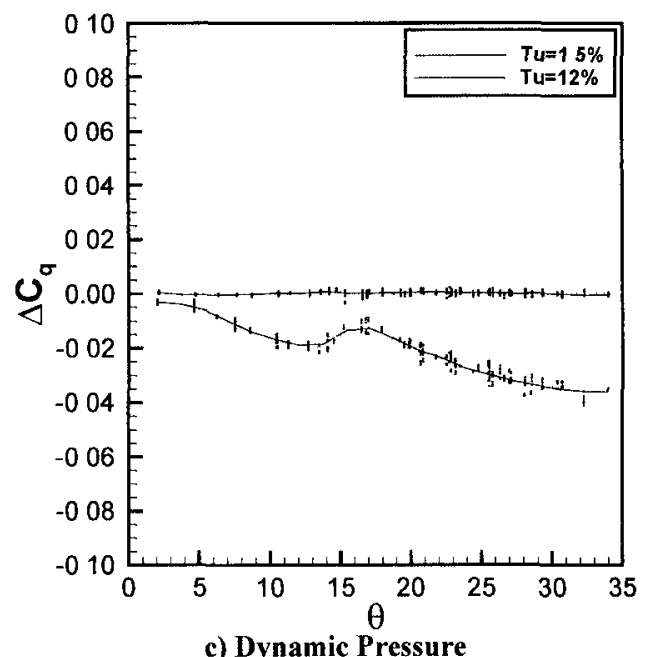

Figure D.31: Seven-Hole Pressure Probe (7HP2) Results: Calibration at $\operatorname{Re}_{\mathrm{D}}=4700, \mathrm{Tu}=1.5 \%$; Data at $\mathrm{Re}_{\mathrm{D}}=4700$, $\mathrm{Tu}$ as shown 

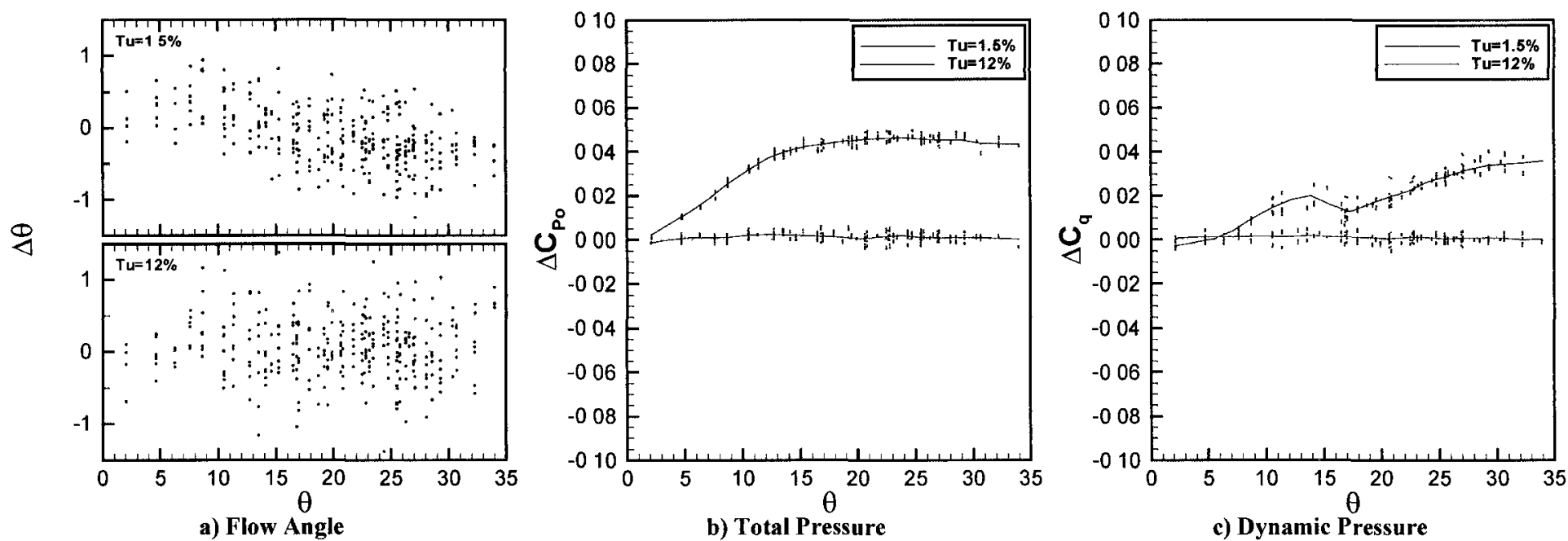

Figure D.32: Seven-Hole Pressure Probe (7HP2) Results: Calibration at $R_{\mathrm{D}}=3200, \mathrm{Tu}=12 \%$; Data at $\mathrm{Re}_{\mathrm{D}}=3200$, $\mathrm{Tu}$ as shown

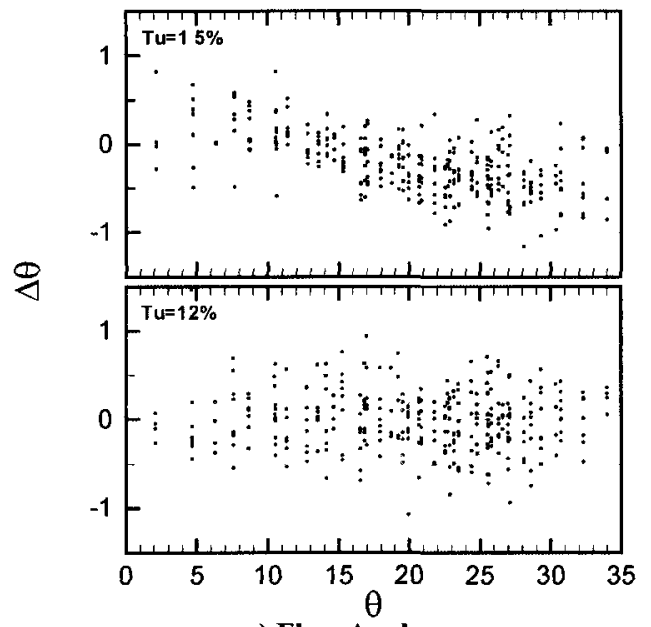

a) Flow Angle

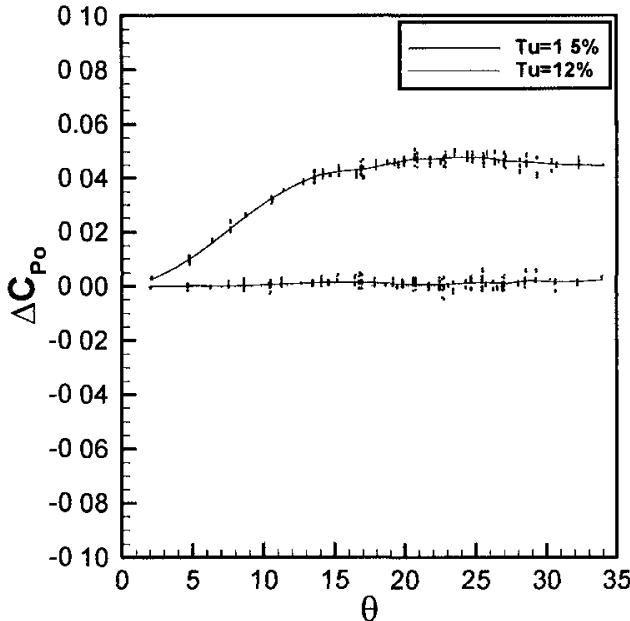

b) Total Pressure

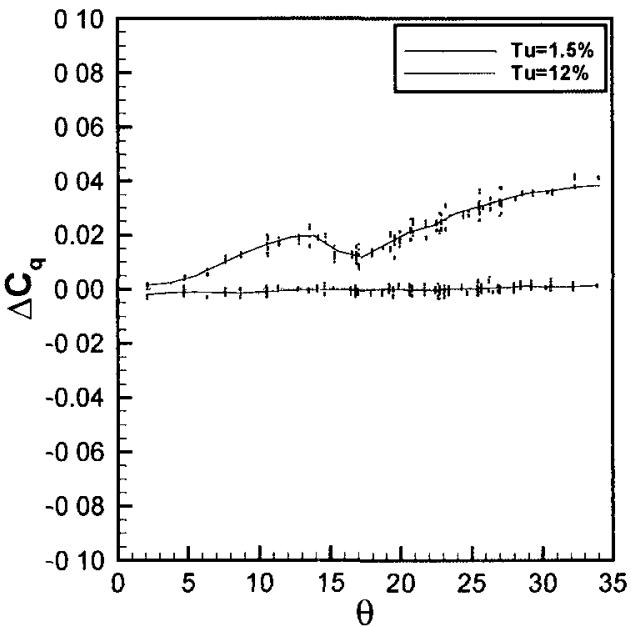

c) Dynamic Pressure

Figure D.33: Seven-Hole Pressure Probe (7HP2) Results: Calibration at $R_{D}=4700, T u=12 \%$; Data at $R_{D}=4700$, $T u$ as shown 

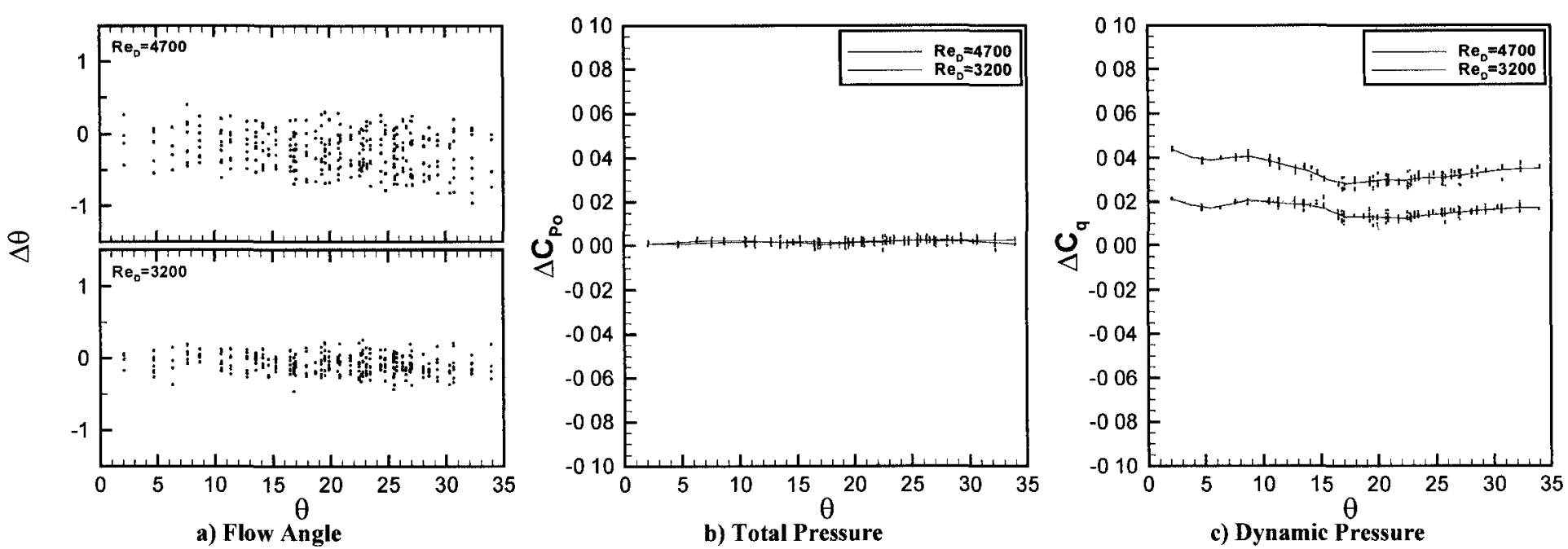

Figure D.34: Seven-Hole Pressure Probe (7HP2) Results: Calibration at $R_{D}=2350, T u=1.5 \%$; Data at $R_{D}$ as shown, $T u=1.5 \%$

9

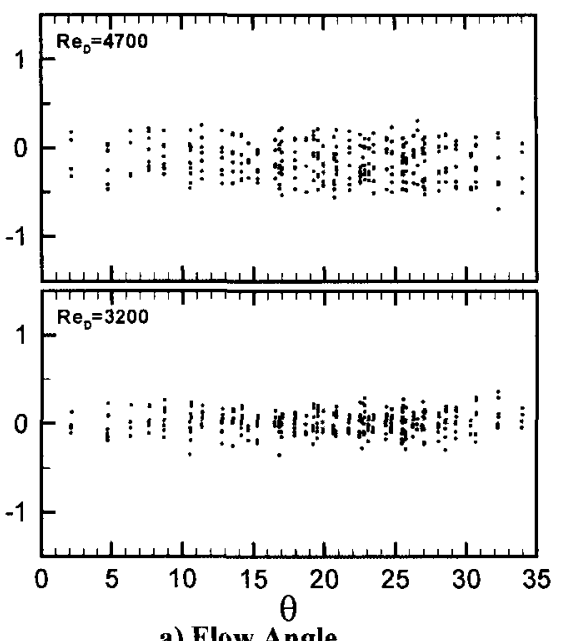

a) Flow Angle

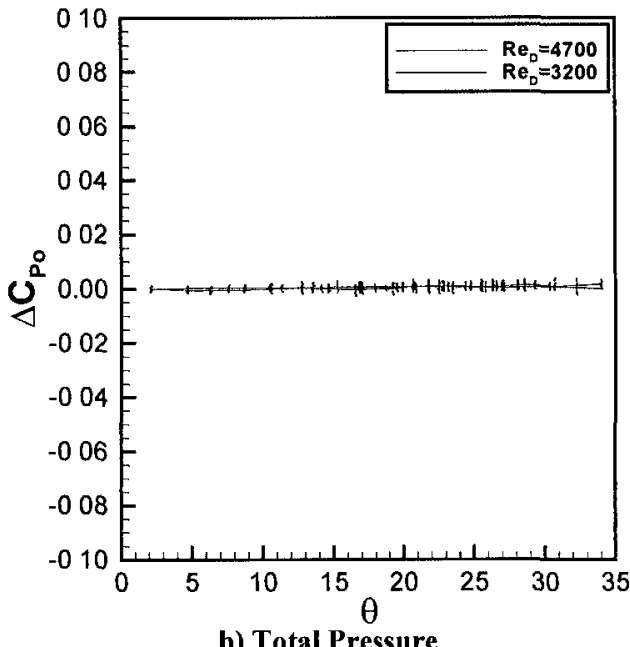

b) Total Pressure

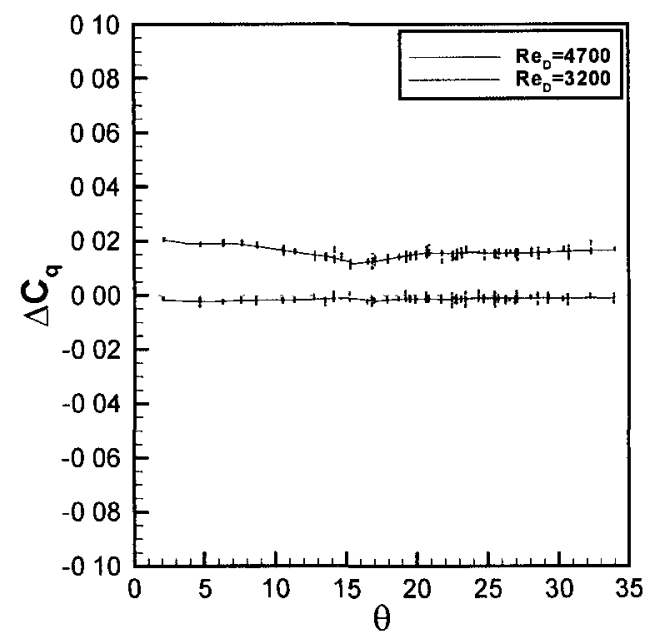

c) Dynamic Pressure

Figure D.35: Seven-Hole Pressure Probe (7HP2) Results: Calibration at $R_{\mathrm{D}}=3200, \mathrm{Tu}=1.5 \%$; Data at $\mathrm{Re}_{\mathrm{D}}$ as shown, $\mathrm{Tu}=1.5 \%$ 

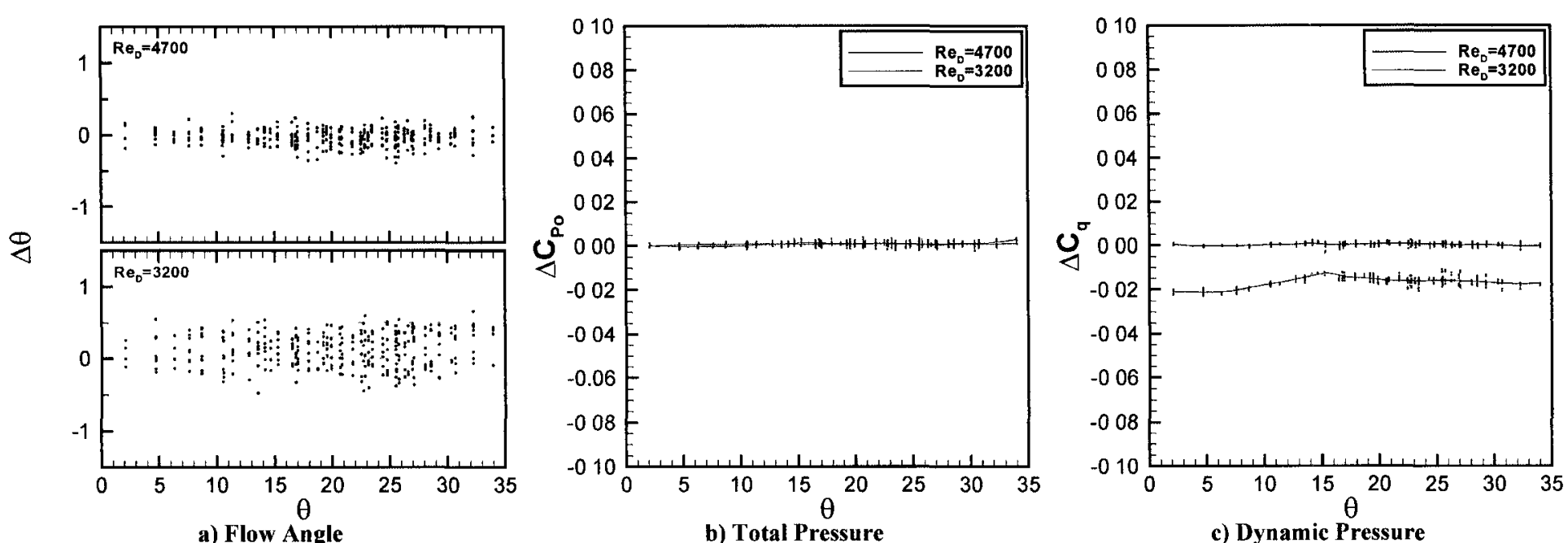

Figure D.36: Seven-Hole Pressure Probe (7HP2) Results: Calibration at $\operatorname{Re}_{\mathrm{D}}=4700, \mathrm{Tu}=1.5 \%$; Data at $\mathrm{Re}_{\mathrm{D}}$ as shown, $\mathrm{Tu}=1.5 \%$

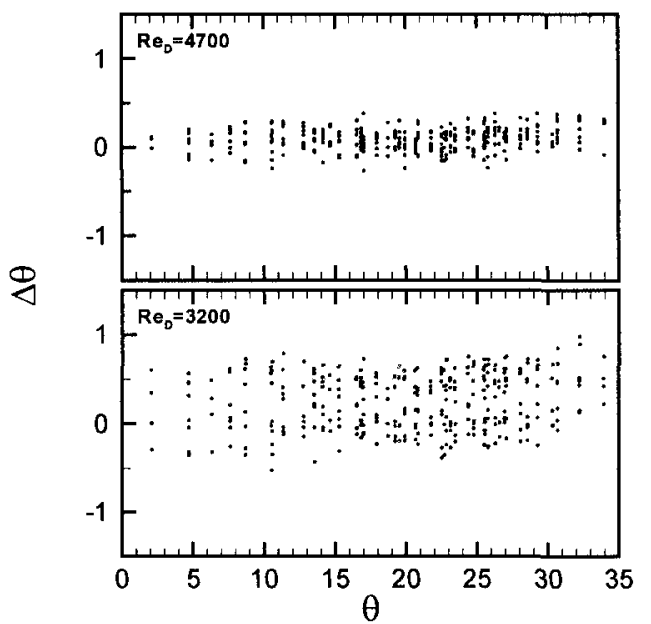

a) Flow Angle
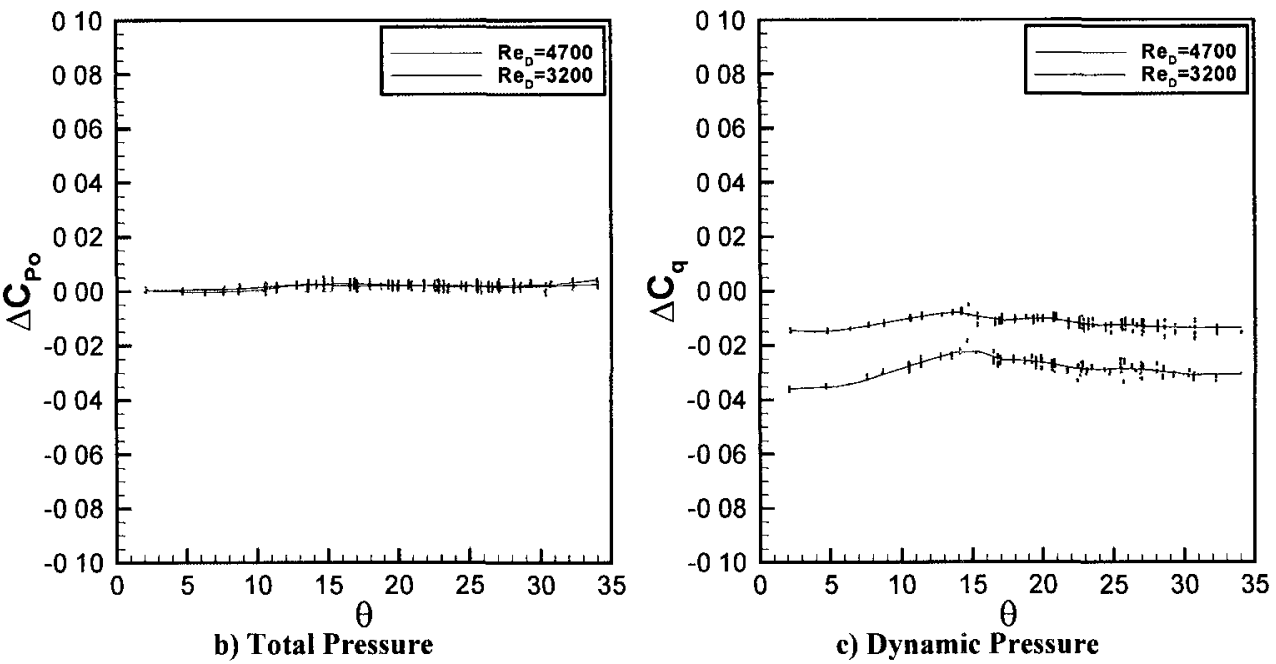

Figure D.37: Seven-Hole Pressure Probe (7HP2) Results: Calibration at $\operatorname{Re}_{\mathrm{D}}=6400, \mathrm{Tu}=1.5 \%$; Data at $\mathrm{Re}_{\mathrm{D}}$ as shown, $\mathrm{Tu}=1.5 \%$ 

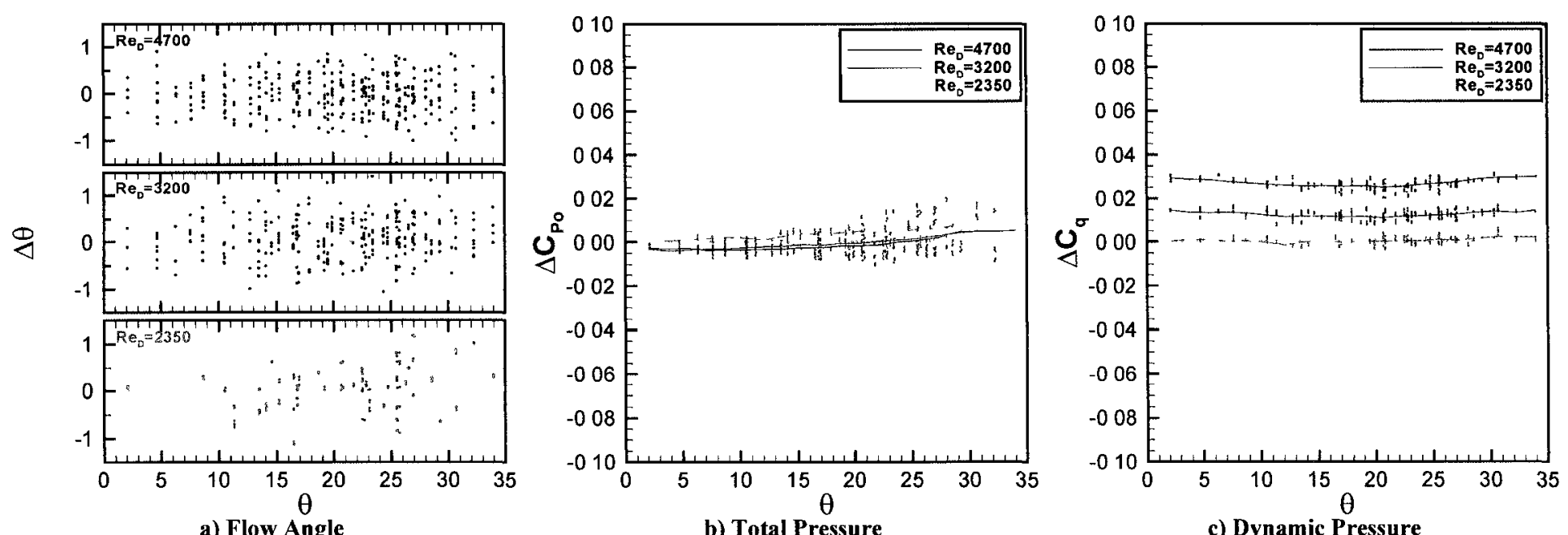

Figure D.38: Seven-Hole Pressure Probe (7HP2) Results: Calibration at $\operatorname{Re}_{\mathrm{D}}=\mathbf{2 3 5 0} \mathrm{Tu}=12 \%$; Data at $\operatorname{Re}_{\mathrm{D}}$ as shown, $\mathrm{Tu}=12 \%$
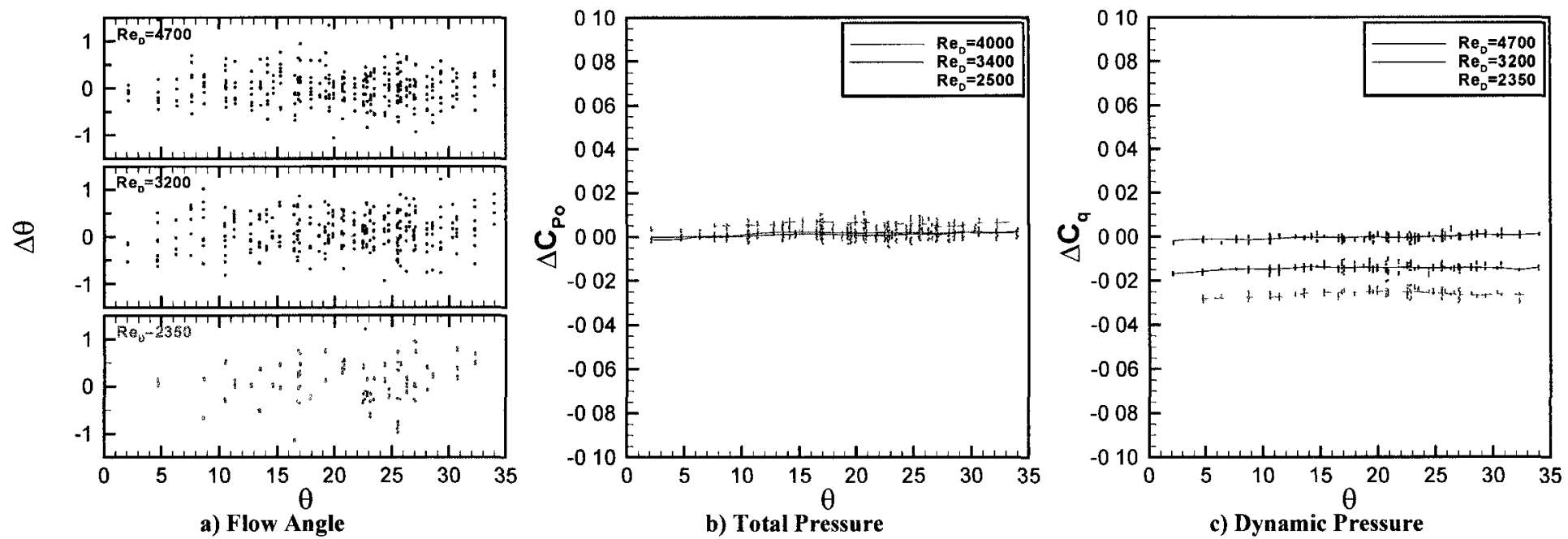

Figure D.39: Seven-Hole Pressure Probe (7HP2) Results: Calibration at $\operatorname{Re}_{D}=4700, T u=12 \%$; Data at $\operatorname{Re}_{D}$ as shown, $T u=12 \%$ 


\section{Appendix E:}

\section{Bi-Linear Interpolative Data Reduction}

\section{Procedure for Multi-Hole Pressure Probes}

The present author has extended the interpolative data reduction approach of MacIsaac et al. (2009) to account for the effects both turbulence intensity and Reynolds number on wind tunnel data collected using multi-hole pressure probe. This new data reduction procedure involves calibrating the probe at several operating points over the full range of turbulence intensities and Reynolds numbers investigated and reducing each data point using a bi-linear interpolation scheme between the these calibrations. The present study employed Delaunay Triangulation for the interpolation, but other interpolation schemes may also be suitable. The results of this process were iterated on

for dynamic pressure (and thus Reynolds number) in order to determine the flow properties at each data point. The turbulence intensity for each data point was obtained independently from the multi-hole probe measurements prior to data reduction. 